\title{
Fatty acid and glucose metabolism in upper body obesity : effects of diet and exercise compared to pioglitazone administration
}

Citation for published version (APA):

Shadid, S. (2007). Fatty acid and glucose metabolism in upper body obesity : effects of diet and exercise compared to pioglitazone administration. [Doctoral Thesis, Maastricht University]. Universitaire Pers Maastricht. https://doi.org/10.26481/dis.20070523ss

Document status and date:

Published: 01/01/2007

DOI:

10.26481/dis.20070523ss

Document Version:

Publisher's PDF, also known as Version of record

Please check the document version of this publication:

- A submitted manuscript is the version of the article upon submission and before peer-review. There can be important differences between the submitted version and the official published version of record.

People interested in the research are advised to contact the author for the final version of the publication, or visit the DOI to the publisher's website.

- The final author version and the galley proof are versions of the publication after peer review.

- The final published version features the final layout of the paper including the volume, issue and page numbers.

Link to publication

\footnotetext{
General rights rights.

- You may freely distribute the URL identifying the publication in the public portal. please follow below link for the End User Agreement:

www.umlib.nl/taverne-license

Take down policy

If you believe that this document breaches copyright please contact us at:

repository@maastrichtuniversity.nl

providing details and we will investigate your claim.
}

Copyright and moral rights for the publications made accessible in the public portal are retained by the authors and/or other copyright owners and it is a condition of accessing publications that users recognise and abide by the legal requirements associated with these

- Users may download and print one copy of any publication from the public portal for the purpose of private study or research.

- You may not further distribute the material or use it for any profit-making activity or commercial gain

If the publication is distributed under the terms of Article 25fa of the Dutch Copyright Act, indicated by the "Taverne" license above, 


\section{Fatty Acid and Glucose Metabolism in Upper Body Obesity}

Effects of diet and exercise compared to pioglitazone administration 
Cover illustration: “"Fatal Attraction" - Cholesterol Crystals in Blood' by Samyah Shadid Cover design: Samyah Shadid in cooperation with Datawyse

Scientific studies presented in this thesis were financially supported by grants DK40484, DK45343, DK50456 and R00585 from the U.S. Public Health Service and by the Mayo Foundation. Further support was provided by NovoNordisk Pharma

Printing of this thesis was financially supported by Eli Lilly Pharma

Printed by: Datawyse / Universitaire Pers Maastricht

ISBN 9789052786285

(C) S.Shadid, Venlo 2007 


\section{Fatty Acid and Glucose Metabolism in Upper Body Obesity}

\section{Effects of diet and exercise compared to pioglitazone administration}

\section{PROEFSCHRIFT}

ter verkrijging van de graad van doctor aan de Universiteit Maastricht, op gezag van de Rector Magnificus, Prof. mr. G.P.M.F. Mols, volgens het besluit van het College van Decanen,

in het openbaar te verdedigen op woensdag 23 mei 2007 om 14:00 uur

door

\section{Samiah Shadid}

Geboren op 14 januari 1970 te Leiden

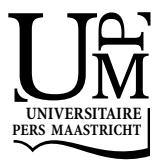




\section{Promotores}

M.D. Jensen, MD, (Mayo Medical School, MN, USA)

Prof. dr. CDA Stehouwer

\section{Beoordelingscommissie}

Prof. dr. ir. A .M.W.J. Schols (voorzitter)

KN Frayn, PhD MRCPath (Oxford University)

Prof. dr.ir. WHM Saris

Prof. dr. N C Schaper

Prof. dr. ir. J.C. Seidell (Vrije Universiteit Amsterdam) 
Nearly everything you do is of no importance, but it is important that you do it. - Mahatma Gandhi 



\section{Contents}

Chapter 1 Introduction 9

1.1 The Obesity Epidemic 11

1.2 Outline of this Thesis 12

Chapter 2 Physiology of Adipose Tissue and Fat Metabolism 15

$\begin{array}{lll}2.1 & \text { Physiology of Adipose Tissue and Fat } & 17\end{array}$

2.2 Direct Free Fatty Acid Uptake into Human Adipocytes In Vivo-

Relation to Body Fat Distribution

Diabetes 2007 Feb 7; [Epub ahead of print]

2.3 Basal and Insulin Regulated Free Fatty Acid and Glucose Metabolism in Humans

Am J Physiol Endocrinol Metab. 2007 Feb 13; [Epub ahead of print]

Chapter 3 Obesity 45

3.1 Definitions and Adverse Effects Of Obesity 47

3.2 Pathophysiology: Effect of Obesity on Endocrine Control of Nutrient

Partitioning

From: 'Obesity: Clinical Evaluation and Treatment', Lippincott, Williams \& Wilkins, 2003

Chapter 4 Treatment Of Upper Body Obesity and Insulin Resistance $\quad 75$

$\begin{array}{lll}4.1 & \text { Introduction } & 77\end{array}$

$\begin{array}{lll}4.2 & \text { Testosterone Administration } & 78\end{array}$

4.3 Effects of Growth Hormone Administration in Human Obesity
Review Article: Obesity Research 2003; 11:170-175

4.4 Insulin Sensitization $\quad 86$

4.4.1 Energy Restriction and Physical Activity Training 86

4.4.2 Pharmacological Insulin Sensitizers 86

4.4.3 Effect of Pioglitazone on Biochemical Indices of Non-Alcoholic Fatty Liver Disease in Upper Body Obesity

Clinical Gastroenterology and Hepatology Sept 2003; 1 (5): 384-7 89

4.5 Pioglitazone Treatment Contrasted to Diet andExercise 94

$\begin{array}{lll}\text { 4.5.1 Aims of Comparison } & 94\end{array}$

4.5.2 Methods of Research 94 
4.5.3 Effects of Diet and Exercise versus Pioglitazone on Free Fatty Acid Kinetics: Pioglitazone increases FFA Clearance Diabetologia. 2006 Jan;49(1):149-57. Epub 2005 Dec 2

4.5.4 Diet/Exercise Versus Pioglitazone: Effects of Insulin Sensitization with Decreasing or Increasing Fat Mass on Adipokines and Inflammatory Markers

JCEM 2006 Sep;91(9):3418-25. Epub 2006 Jun 27

4.5.5 Treatment of Obesity with Diet/Exercise versus Pioglitazone has Distinct Effects on Lipoprotein Particle Size Atherosclerosis 2006, Oct;188(2):370-6. Epub 2005 Nov 28

4.5.6 Effects of Diet and Exercise Versus Pioglitazone on Metabolic Health and Fat Distribution in Upper Body Obesity

Diabetes Care 26:3148-3152, 2003

Chapter 5 Summaries and Future Perspectives 137

5.1 Summary and Future Perspectives 139

5.2 Nederlandse Samenvatting (Summary in Dutch) 153

$\begin{array}{ll}\text { References } & 158\end{array}$

$\begin{array}{ll}\text { Appendices } & 177\end{array}$

1 Abbreviations 177

2 Conversion Tables $\quad 178$

3 Exercise Program 178

4 Behavior Modification Program 179

Acknowledgements/ Dankwoord $\quad 180$

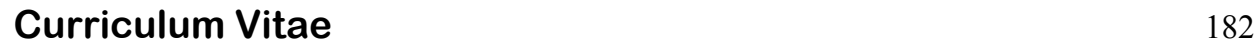




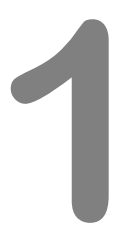

\section{Introduction}


1.2 Outline of this Thesis 


\subsection{The Obesity Epidemic}

Overweight is a steadily increasing threat to public health. In the USA it is currently the number 2 cause of preventable death (1); $64.5 \%$ of adults (i.e. almost 100 million people) are overweight (body mass index $>25$; see table 3.1 .1 ), whereas $30.5 \%$ have a body mass index over 30 and therefore qualify as obese (2).

In other countries, these percentages average lower but are progressively rising. In the Netherlands for instance, the most recent percentages from 1998 show that $11 \%$ of men and $12 \%$ of women were obese. In Britain, this was 10 and 17\% respectively. However, if the same trend is followed as in the United States, percentages are expected to be substantially higher in 2006 (figures 1.1 and 1.2).

Figure 1.1 Worldwide prevalence of obesity. Based on data (1987-1994) released by the International Obesity Task Force (IOTF) in 1997.

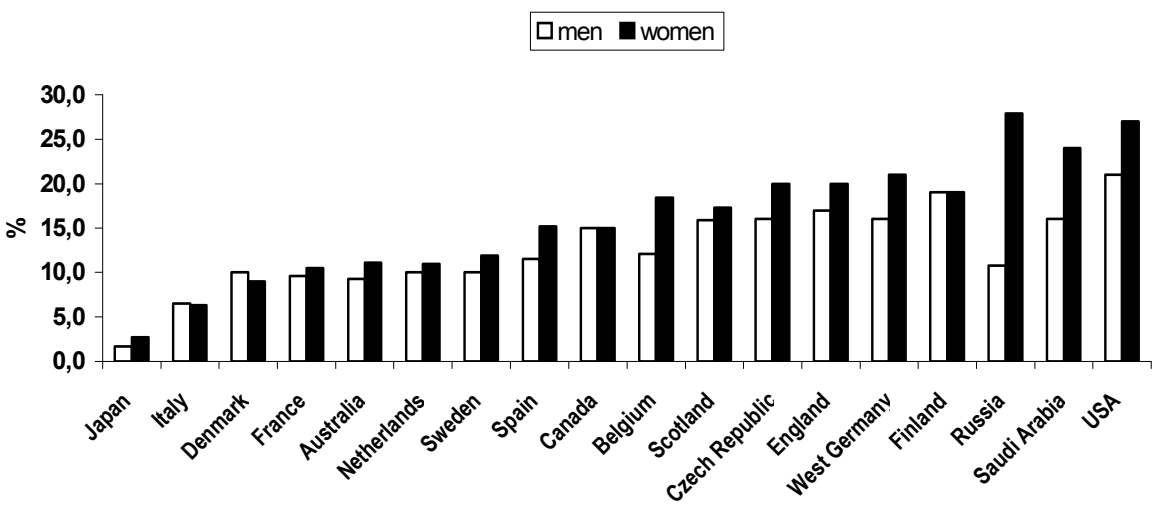

The rising incidence in children is particularly worrying. 16\% of American (NHANES 19992002), 14\% of Dutch (Gezondheidsraad, 2003) and 17\% of British children (UK Office for National Statistics and Health, 2002) are reported to be overweight or obese. This will not only increase the prevalence of adult obesity, but also the cumulative exposure and thus the incidence of obesity-related complications (see Chapter 3). This could greatly challenge the volume and costs of future health care.

Estimates are that, in $2025,40 \%$ of the American and $25 \%$ of the adult English population will have a body mass index over 30 .

Prevention of obesity would therefore be invaluable. However, this requires population-based measures to change life style, which are difficult to implement and, perhaps therefore, still largely absent. The same is true for obesity treatment on a large scale: behavior modification programs and specific, individualized dietary and exercise guidelines are labor-intensive, expensive, largely dependent on multi-disciplinary approaches.

Consequently, prevention and therapy of obesity should be supported by pathophysiological research in order to minimize its complications as long as fat mass cannot be reduced. 
Figure 1.2 Obesity trends in US Adults. Sources: Mokdad A H, et al. J Am Med Assoc 1999; 282: 16, and JAMA 2001; 286:10 and publications of the Center of Disease Control and Prevention

1985

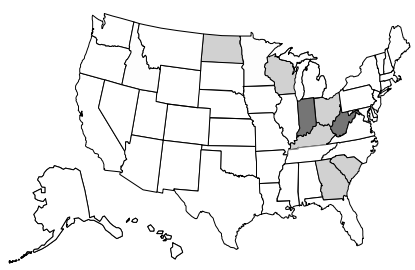

1999

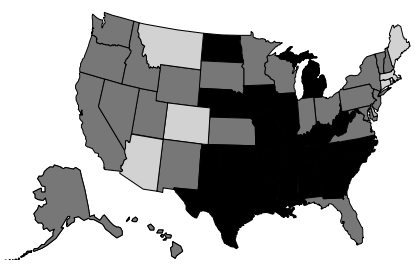

$<10 \%$ or No data

$10-14 \%$

$15-19 \%$

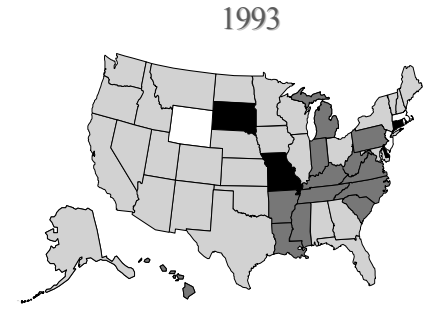

2005

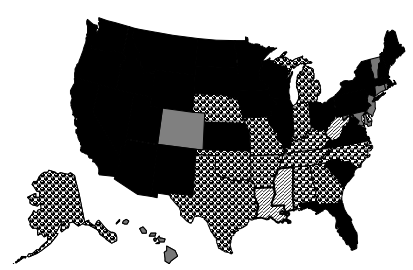

$20-24 \%$ 圈 25-29\% 团 $30-34 \%$

\subsection{Outline of this thesis}

Since one of the major complications of obesity is insulin resistance (Chapter 3.1), increasing insulin sensitivity plays a central role in preventing obesity-related complications, and has, therefore, been the focus of our investigations and this thesis.

First, we describe the physiology of fatty acids and adipose tissue (Chapter 2).

In paragraph 2.2, we also describe an original study in which we test whether free fatty acid uptake can occur without the intervention of VLDL particles, and to what extent this might influence (gender related) body fat distribution. In Chapter 2.3 we report on the main determinants of adipose tissue lipolysis/FA release in lean and obese volunteers under basal and hyperinsulinemic clamp conditions. Chapter 3 defines obesity and its metabolic consequences. The last section (Chapter 3.2) shall focus on fuel trafficking and metabolism, endocrine control hereof and the effect of obesity on these processes.

Subsequently, treatment options in upper body obesity shall be discussed in Chapters 4.1 to 4.3 , including two literature reviews on the effects of testosterone and growth hormone administration in upper body obesity. We shall then (Chapter 4.5) compare the effects of two methods of insulin sensitization (pioglitazone and diet/exercise) amongst each other qualitatively and quantitatively (Chapters 4.5.1-4.5.7) with respect to their direct effects on meta- 
bolic parameters, body composition, fatty acid metabolism, energy expenditure and adipokine concentrations.

Chapters 5 (English) and 6 (Dutch) summarize the thesis and discuss future perspectives. 



\section{Physiology of Adipose Tissue and Fat Metabolism}


2.1 Physiology of Adipose Tissue and Fat 17

$\begin{array}{ll}\text { 2.1.1 Fatty Acid and Triglyceride Metabolism } & 17\end{array}$

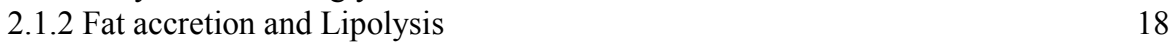

2.1.3 Regional Differences in Fat Depots: consequences of Body Fat Distribution 18

2.1.4 Adipose Tissue as an Endocrine Organ 19

2.2 Direct Free Fatty Acid Uptake into HumanAdipocytes in

Vivo - Relation to Body Fat Distribution

Diabetes2007 Feb 7; [Epub ahead of print]

2.3 Basal and Insulin Regulated Free Fatty Acid and Glucose Metabolism in Humans

Am J Physiol Endocrinol Metab. 2007 Feb 13; [Epub ahead of print] 


\subsection{Physiology Of Adipose Tissue And Fat}

\subsubsection{Fatty Acids and Triglycerides}

Fatty acids (FAs) circulate in plasma either bound to albumin, as 'free fatty acids' (FFAs), or within lipoproteins, as components of triglycerides. Dietary triglycerides are taken up in the small intestine and transported in chylomicrons to peripheral tissues, where the triglycerides are hydrolyzed by Lipoprotein Lipase (LPL), thus liberating FFA for storage or oxidation. The chylomicron remnants, which are still rich in TG and cholesterol, are largely taken up by the liver.

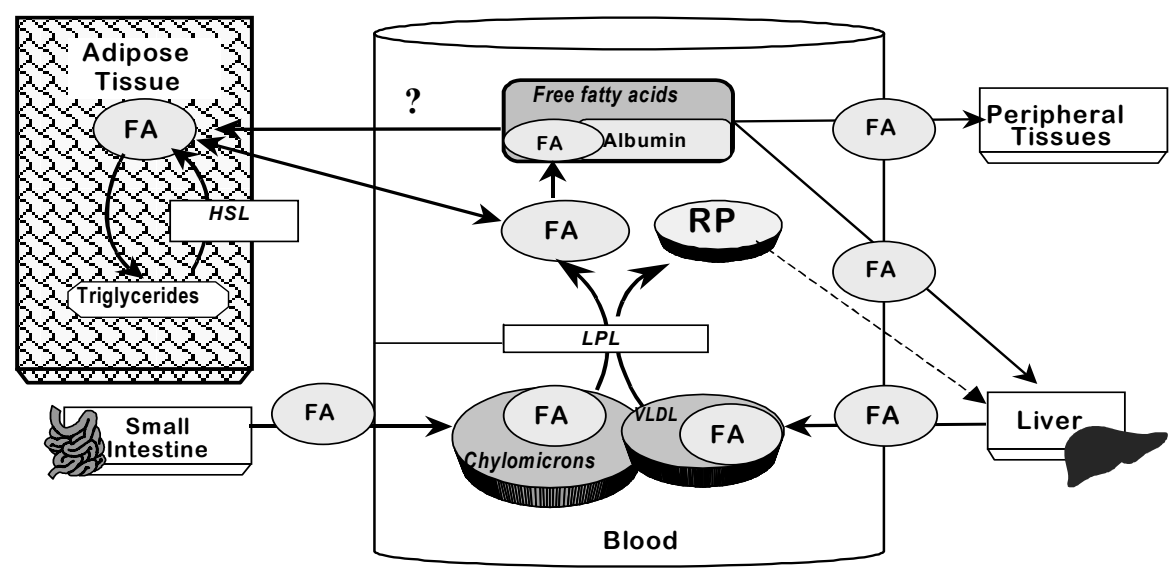

Figure 2.1 Kinetics of Fatty Acids and Triglycerides. Free Fatty Acids are albumin-bound, non-esterified fatty acids (FA). RP= Remnant Particle; HSL= Hormone-Sensitive Lipase; LPL= Lipoprotein Lipase

Some of the endogenous FFA are esterified to triglycerides and secreted by the liver as Very Low Density Lipoprotein (VLDL) for release into the circulation (figure 2.1). The VLDL pathway resembles that of chylomicrons, in that clearance occurs mainly via LPL; however VLDL remnants, so-called IDL (intermediate-density lipoprotein) particles, can either be taken up by the liver or be further freed from TGs in the circulation to form LDL (low density lipoprotein) eventually.

At rest, FFA release and uptake rates generally exceed fatty acid oxidation rates. FFAs circulate at relatively low concentrations $(300-600 \mu \mathrm{mol} / 1$ in the post-absorptive state) and turn over rapidly with a half-life of $\sim 4$ minutes. FFA availability in the circulation is a significant, but not exclusive determinant of whole body fatty acid oxidation: hormonal influences may additionally promote (growth hormone) or inhibit (insulin) intracellular FA oxidation. This concomitantly influences carbohydrate oxidation (Chapter 3.2.1). 


\subsubsection{Fat accretion and lipolysis}

The net balance between fat accretion and lipolysis determines fat compartment volume. Adipose tissue LPL is the main determinant of fat accretion; it is activated by insulin and inhibited by catecholamines, growth hormone and cortisol; to date, it is unknown whether FFAs can also enter AT directly from blood without LPL (see figure 2.1); this will be further discussed in Chapter 2.2. The glycerol skeleton of intra-adipocyte TG is largely derived from glucose.

Figure 2.2 Reciprocal action and regulation of Hormone Sensitive Lipase (HSL) and Lipoprotein Lipase (LPL). ACTH= Adrenocorticotrope Hormone

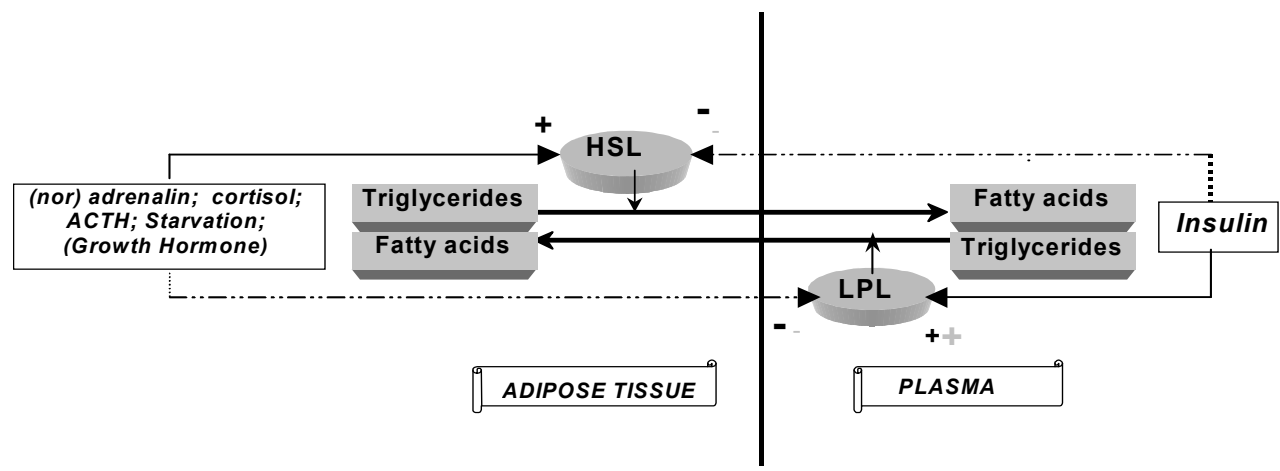

AT lipolysis is mediated by hormone sensitive lipase (HSL). Recently, adipose triglyceride lipase has been identified, a new adipose tissue lipase, which is thought to catalyze the initial step in triglyceride hydrolysis; its relative in vivo contribution to AT lipolysis in humans is, however, not yet known $(3,4)$.

HSL is reciprocally regulated by the same hormones as its anabolic counterpart LPL: epinephrine stimulation of HSL via $\beta$-adrenergic receptors can increase FFA release 3-4 fold (5), whereas insulin is a very effective inhibitor of HSL-mediated lipolysis.

Physiological hyperinsulinemia is capable of suppressing FFA release rates by as much as 80 $90 \%$ from basal levels, and FFA concentrations by $>90 \%$. This action is impaired in insulin resistant, obese individuals.

In vivo, adipose tissue lipolysis may be estimated by glycerol appearance rate in blood or by fatty acid flux (see Chapters 4.5.1 and 4.5.3). After lipolysis, FAs may be re-esterified to TG within the adipocyte, identically to newly taken up FAs. This process is also stimulated by insulin (6-8)

\subsubsection{Regional differences in fat depots: consequences of body fat distribution}

Differentially located fat compartments vary in physiological properties, such as postprandial net fat uptake, lipolytic rates and the reaction to hormones. Body fat distribution may there- 
fore markedly influence whole-body metabolism and thus the metabolic consequences of obesity (9-11).

Excess fat storage may range from preferential accumulation in the gluteo-femoral to preferential storage the abdominal fat depots, resulting in gynoid (pear-shaped, lower-body) and

Figure 2.3 Upper and Lower Body Obesity. Courtesy of MD Jensen, MD

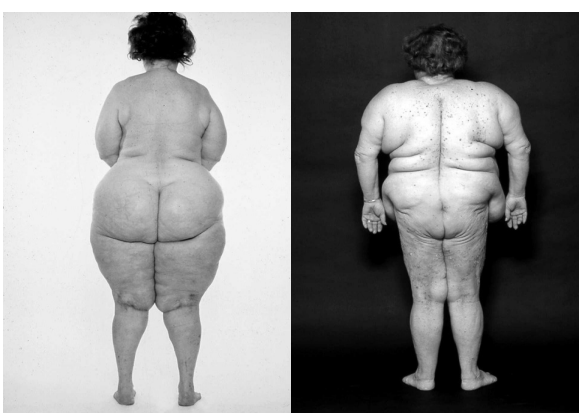

android (apple-shaped, upper body) obesity phenotypes respectively. These two may be clinically distinguished by the waist-to-hip ratio (WHR, i.e waist diameter divided by hip circumference). Cut-off points are $<0.76$ for lower body obesity (LBO) and $>0.85$ for upper body obesity (UBO) for women and $>0.90$ for men is generally defined as upper body obesity. These cut off values for women represent the bottom and top $25^{\text {th }}$ percentile of fat distribution for Caucasian women with Class I-II obesity.

UBO is associated with the metabolic syndrome, more frequently and more severely than LBO $(9,11)$. This has been attributed to the influence of the insulin resistant visceral fat compartment, although this mechanism remains speculative (see Chapter 3.1.2).

What determines fat distribution is unknown. To what extent fat accretion, lipolysis or both are involved in regional fat gain and to what extent hormones, including sex steroids, influence the substantial inter-individual variations remains to be defined. In Chapter 2.2, the contribution of regional differences in FA uptake is studied.

\subsubsection{Adipose Tissue As An Endocrine Organ}

In addition to triglyceride storage, adipose tissue produces several substances, originating from adipocytes, preadipocytes, fibroblasts or other cell types within the tissue. These socalled adipocytokines, or adipokines, possess several autocrine, paracrine and endocrine functions, which are only partly known. Some, but not all of these adipokines are described in Table 2.1.1; not all of these substances, such as Tumor necrosis Factor- $\alpha$ (TNF- $\alpha$ ), angiotensinogen etc, are exclusive to adipose tissue.

Adipokine release from adipose tissue may be regulated by various factors, including fat cell size, catecholamines and classical 'inflammatory' stimuli such as lipopolysaccarides (12). They may also modulate each other's production and/or function: TNF- $\alpha$ stimulates Interleukin-6 (IL-6) production, and IL-6 increases C-reactive protein (CRP) production in the liver.

Grossly, adipokine effects can be classified as trophic (such as the induction of apoptosis and the regulation of cell size), stimulatory or metabolic. The latter effects, including the inhibition of lipoprotein lipase and stimulation of hormone-sensitive lipase, should reduce lipid accumulation within fat (12).

Although several cytokine functions have been well established, it is not entirely clear if and to what extent adipokines act as true endocrine regulators. Nevertheless, some have been implicated in hypertension (angiotensinogen) and impaired fibrinolysis (PAI-1), and others are thought to play a direct role in the pathogenesis of obesity and insulin resistance (see also 
Chapter 4.5.5). The fact that TNF- $\alpha$ and IL-6 are among those, prompted the postulation of these conditions as being states of low-grade inflammation (13).

Table 2.1.1 Some substances produced by adipose tissue $(14,15)$

\begin{tabular}{lll}
\hline Adiponectin & Interleukin-1 $\beta$ & Acylation-stimulating protein (ASP) \\
Resistin & Interleukin-6 & Vascular Endothelial Growth Factor \\
Leptin & Interleukin- 8 & Retinol binding protein 4 \\
Visfatin & Interleukin-10 & Transforming growth factor (TGF)- $\beta$ \\
Tumor Necrosis Factor (TNF)- $\alpha$ & Prostaglandin $\mathrm{E}_{2}$ and $\mathrm{I}_{2}$ & Plasminogen activator inhibitor (PAI)-1 \\
Angiotensinogen & Serum Amyloid A & Insulin-like Growth Factor-I (IGF-I) \\
Lipoprotein Lipase & Hormone Sensitive Lipase & Monobutyrin \\
\hline
\end{tabular}

Another reason for the postulation of a pathophysiological endocrine role of adipokines in obesity and insulin resistance was the discovery that concentrations of various adipokines are changed in these conditions, and that most adipokines are produced in quantities directly proportional to total fat mass (except adiponectin). Moreover, regional differences have been reported in the production of some adipokines, including leptin, resistin and IL-6 (16)

This theory shall further be discussed in Chapter 4.5.5; in order to place the information in context, we shall discuss the most important adipokines associated with obesity in more detail below.

\section{Leptin}

Leptin is produced by adipocytes in quantities paralleling fat mass. It inhibits food intake on hypothalamic levels, but has also been suggested to directly increase energy expenditure and to stimulate peripheral FA oxidation in skeletal muscle and liver cells. Its action on lipolysis is controversial $(14,17)$.

Leptin deficiency or mutations of the leptin receptor lead to hyperphagia (and hence severe obesity), accompanied by metabolic, neuro-endocrine, and immune dysfunction (17). However, a true leptin deficiency as a cause of early onset obesity is extremely rare, and experimental leptin administration in larger groups of obese humans has been disappointing $(17,18)$. On the contrary, most obese subjects appear to have high plasma concentrations of leptin. More likely, the importance of leptin seems to lie in underweight, in which decreasing concentrations stimulate hyperphagia.

\section{TNF- $\alpha$ and IL-6}

TNF- $\alpha$, Il-6 and resistin are thought to induce insulin resistance, although their in vivo endocrine relevance in humans remains controversial.

TNF- $\alpha$ is produced by adipose tissue and is closely linked to adipocyte metabolism as a paracrine factor. It is suggested to modify lipid synthesis and lipolysis, specifically in skeletal muscle and adipocytes. In vitro, increasing fat cell size increases TNF- $\alpha$ production, which in turn, inhibits adipocyte size expansion via negative feedback mechanisms (19).

There are, however, very few in vivo reports of this correlation (see Chapter 4.5.5). More importantly in this context, the in vivo association of TNF- $\alpha$, obesity, insulin resistance and insulin sensitization is very variable. Evidence for a true causal association between TNF- $\alpha$ and the development of whole-body insulin resistance has therefore been limited to studies of obese mice (17) and in vitro experiments (20).

IL-6 seems to play a more complex role in insulin resistance. Although some suggest a pivotal role (21), IL-6 is generally thought to induce insulin resistance, perhaps by increasing AT 
lipolysis (22). It also elicits a pronounced vasodilatation, and markedly increases the uptake of fatty acids and the gluconeogenic precursors glycerol and lactate. However, its role in mediating insulin resistance might be epiphenomenal and the controversy in the evidence hereon is not surprising considering its role as a general inflammatory marker, like TNF- $\alpha$ and CRP.

\section{Resistin}

Resistin is a peptide specific to AT and has been demonstrated in mice and humans (23). Despite circumstantial evidence such as regional variability in resistin production (higher in abdominal subcutaneous and omental beds than in thigh depots) (23), its clinical role in insulin resistance has not yet been proven $(23,24)$. Inconsistent in vivo findings of its plasma concentrations in insulin resistant and diabetic humans challenges theories regarding its function in inducing or sustaining a systemic disorder (Chapter 4.5.5).

\section{C-reactive protein}

Although C-reactive protein (CRP) is not produced by fat, it is many times referred to in the same context as adipokines, not only because its concentrations are, among others, increased by stimulation by TNF- $\alpha$ and IL-6 (12), but also because CRP concentrations are elevated in obesity and insulin resistance/ type 2 diabetes mellitus $(25,26)$. However, smoking, plasma lipids (especially HDL cholesterol), hypertension, and measures of subclinical atherosclerotic disease (e.g. ankle-brachial blood pressure index) are also correlates of CRP.

This complicates the investigation of its pathogenetic role in mediating insulin resistance. Nonetheless, BMI, waist-to-hip ratio and insulin sensitivity have been demonstrated to correlate with CRP in vivo (27-32), and CRP predicts future cardiovascular events independently of more 'traditional' risk factors, such as plasma lipid levels (30). Some have therefore suggested the use of CRP in clinical practice as a predictor of cardiovascular risk. This, however, has not yet been validated and there are still many uncertainties surrounding CRP, such as an explanation for the fact that women have higher CRP values than men at similar BMI and insulin sensitivity levels (32).

\section{Adiponectin}

Adiponectin is an adipocyte-specific secretory protein of which the concentrations are inversely correlated with the degree of adiposity. Adiponectin is thought to enhance insulin sensitivity, one theory being that it increases fat oxidation and therewith reduces both intracellular triglyceride contents in liver and muscle and circulating FA levels (33). In animal models, indeed, reducing adiponectin expression is associated with insulin resistance, and adiponectin administration with increased insulin sensitivity $(33,34)$.

Nonetheless, its exact role in insulin sensitivity is unclear, especially since decreased plasma adiponectin levels are not only found in insulin resistance, but also in coronary artery disease; they may be increased in chronic renal failure, type 1 diabetes and anorexia nervosa (33). Moreover, like in CRP, a relatively consistent gender difference in adiponectin concentrations is reported, being higher in females than in males $(35,36)$.

In summary, an emerging body of evidence suggests that (abdominal) fat is a key regulatory site for the general processes of inflammation, coagulation, and fibrinolysis. These processes may be altered by behaviors, such as diet and exercise, that affect fat deposits, as well as by medications. Their endocrine effect on whole-body insulin resistance, however, remains unclear.

This, and the effects of insulin sensitization on adipokine concentrations and dynamics, are further studied and described in Chapter 4.5.5. 
Chapter 2 


\title{
2.2 Direct Free Fatty Acid Uptake into Human Adipocytes In Vivo - Relation to Body Fat Distribution
}

\author{
S. Shadid, C.Koutsari ${ }^{1}$ and M.D. Jensen, Diabetes. 2007 Feb 7; [Epub ahead of print] \\ Endocrine Research Unit, Mayo Clinic, Rochester, Minnesota, USA
}

\begin{abstract}
We report that direct uptake of circulating free fatty acids (FFA) into adipocytes occurs in vivo, in humans. After validating a method to measure direct adipocyte FFA uptake we measured this process in normal weight, postabsorptive men and women. Direct FFA uptake was greater in women than men $-8.2 \pm 0.6 \%$ vs. $4.0 \pm 0.5 \%(\mathrm{P}<0.0001)$ of FFA were taken up by subcutaneous adipose tissue, respectively. Abdominal subcutaneous fat took up FFA more avidly than femoral fat in men, but this did not occur in women. Similar sex and regional difference were found to occur in obese women and men. These findings are uniquely consistent with the greater subcutaneous body fat mass and the lower body fat distribution in women. Gene expression of fatty acid transporters was greater in abdominal than femoral fat in men (P $<0.05)$, but not in women $(\mathrm{P}=0.80)$. We conclude that sex and site specific recycling of circulating FFA into subcutaneous fat is a novel FFA disposal pathway that may also play a role in the development or maintenance of body fat distribution. Regional variations in facilitated fatty acid transport may contribute to this process.
\end{abstract}

\footnotetext{
${ }^{1}$ Drs Shadid and Koutsari contributed equally to this paer
} 


\section{Introduction}

Body fat distribution is an important predictor of obesity-related disease risk. A visceral/upper body fat distribution often results in elevated plasma FFA concentrations $(37,38,38)$, which can cause insulin resistance and associated metabolic complications (39). How people can differ so remarkably in body fat distribution is not yet known, but regional differences in fatty acid uptake or FFA release between various fat depots almost certainly play a role. Studies of isolated adipocytes or adipose tissue fragments have suggested that regional differences in lipolysis (40) or uptake of triglycerides via lipoprotein lipase (LPL) (41) account for the differences in body fat distribution. Unfortunately, in vivo studies have not been able to detect the differences regional in lipolysis or circulating triglyceride uptake that would be predicted on the basis of in vitro findings.

For example, lean and obese men and women with differing fat distributions do not display the variations in regional FFA release that would be expected to cause retention of fat in certain depots $(42,43,44)(45)$. Greater regional fat accumulation is associated with increased, not reduced lipolysis rates from that depot $(43,44,47)$. Although women have greater femoral adipose tissue lipoprotein lipase activity (LPL) than men (48) in vivo studies from Mårin et al $(49,50,51)$ and our laboratory $(52,53,54)$, have shown that they do not preferentially store dietary fat in this region, at least in the short term. Upper body subcutaneous fat takes up meal fatty acids more efficiently than lower body fat in both women and men (49,52,53-55). Finally, the efficiency of dietary TG uptake into subcutaneous fat is not greater in women than men $(52,54)$, even though women have much more subcutaneous adipose tissue. Thus, human in vivo studies of regional fatty acid metabolism have not yet yielded findings that might explain how humans develop or maintain regional variations in fat accumulation.

These studies were designed to look for the existence of a novel pathway of adipocyte fatty acid uptake - direct uptake of circulating FFA into adipose tissue independent of the LPL mechanism that acts on chylomicron and VLDL triglycerides (TG). This pathway of direct FFA uptake has been recently reported to exist in the postprandial state (Bickerton et al, Diabetes 56:168-176, 2007) and during glucose infusion (298), but it has not been detected in the postabsorptive state (298). The results overturned our perception that adipose tissue does not take up FFA directly in vivo and suggest that the direct FFA uptake process may be important in regulating or maintaining body fat distribution.

\section{Materials and Methods}

Three studies were conducted. The first was to define the optimal approach of measuring direct FFA uptake into adipose tissue. The second was to quantitate direct FFA uptake by adipose tissue in non-obese men and women and the third study was to determine total (direct and VLDL-TG derived) adipose tissue FFA uptake in obese men and women. Each protocol was approved by the Mayo Clinic IRB and informed, written consent was obtained from the volunteers.

\section{Subjects}

All participants were weight stable for at least 3 months prior to the study. Inclusion criteria are described below; exclusion criteria included smoking, pregnancy, breast-feeding and use of medication that could affect lipid metabolism that could not be stopped for at least 2 weeks prior to the study. 


\section{Study Protocols}

Procedures common to all protocols: Each volunteer consumed an isoenergetic diet (50\% carbohydrates, $35 \%$ fat, $15 \%$ protein) from the Mayo General Clinical Research Center (GCRC) for at least 5 days prior to the study. Body composition was measured with a single slice abdominal CT ( $\mathrm{L}_{2-3}$ interspace) and dual energy x-ray absorptiometry (DEXA, DPX-IQ, Lunar Radiation, Madison, WI). After admission to the Mayo GCRC, a forearm vein catheter was inserted and used for tracer infusion and a second catheter was placed in a retrograde fashion in a hand vein for collecting arterialized blood using the heated hand vein technique.

\section{Protocol 1: Distribution of the FFA tracer in adipose tissue lipid fractions}

Five lean and obese women and 7 lean and obese men participated in studies to assess the distribution of the FFA tracer in plasma and in adipose tissue lipids. The time course of the decay of plasma FFA radioactivity and appearance of FFA in plasma TG was determined by collecting blood samples before and at 1, 5, 10, 15, 30, 45 and 60 minutes after a bolus of radiolabeled palmitate. Abdominal and femoral subcutaneous fat biopsies were collected 15 or 30 minutes after the FFA tracer bolus. The tracer distribution in lipids (phospholipids, monoglycerides, diglycerides, TG, cholesterol esters and FFA) extracted from whole adipose tissue and from isolated adipocytes was assessed to distinguish whether the tracer in whole adipose tissue represented intracellular TG or extracellular radiolabeled FFA.

\section{Protocol 2: LPL independent adipose tissue FFA uptake}

This study was to quantitate the direct adipose tissue uptake of circulating FFA in normal weight men and women. We gave an intravenous bolus of an FFA tracer and performed fat biopsies timed such that virtually no FFA tracer remained in the circulation and there would be insufficient tracer in VLDL-TG to allow adipose tissue accumulation via the LPL pathway. Twenty-seven (12 men and 15 women) participated in this study. The morning after admission to the Mayo GCRC adipose tissue blood flow (14) was measured in the overnight postabsorptive state to test for site or sex differences that could affect tissue FFA delivery. The volunteers continued the same diet while staying in the GCRC until the following morning, when after an overnight fast an intravenous bolus of $\left[1-{ }^{14} \mathrm{C}\right]$ oleate was given. Blood samples were collected as in Protocol 1 to measure the decay of ${ }^{14} \mathrm{C}$ oleate in plasma and the appearance of ${ }^{14} \mathrm{C}$ oleate in plasma TG. The adipose biopsies were timed such that the abdominal and femoral fat was collected between 40 - 45 minutes after the tracer bolus.

\section{Protocol 3: Regional and sex differences in adipose tissue FFA uptake in obesity}

This experiment allowed us to examine the cumulative re-entry of plasma FFA into adipose tissue of obese men and women through the direct uptake and indirect (VLDL) routes. Ten obese men and twelve obese premenopausal women participated in this study. The volunteers were admitted to the GCRC the evening prior to the experiment and consumed a standardized evening meal at $1800 \mathrm{hr}$. At $0300 \mathrm{hr}$ a constant infusion of $\left[1-{ }^{14} \mathrm{C}\right]$ palmitate $(\sim 0.3 \mu \mathrm{Ci} / \mathrm{min})$ was started and at $0900 \mathrm{hr}$ femoral and abdominal subcutaneous adipose tissue biopsies were obtained.

\section{Materials}

$\left[1-{ }^{14} \mathrm{C}\right]$ palmitate, $\left[9,10-{ }^{3} \mathrm{H}\right]$ palmitate and $\left[1-{ }^{14} \mathrm{C}\right]$ oleate were purchased from NEN Life Science Products, PerkinElmer, Boston, MA. $\left[{ }^{133} \mathrm{Xe}\right]$ (Syncor Corp, St. Paul, MN) was dissolved in sterile saline and used to measure regional adipose tissue blood flow (52-54). 


\section{Assays and Methods}

Plasma concentrations and SA Plasma palmitate and oleate concentrations and SA were measured using HPLC (56-58). To measure the entry of the FFA tracer into plasma triglyceride, lipids were extracted from a $1.0 \mathrm{~mL}$ plasma sample using the Dole procedure and FFA were separated from triglycerides using a modification (42) of a published HPCL procedure (59). The plasma TG concentrations were measured (60) the counts in the TG fraction of the HPLC separated plasma used to calculate the TG SA.

Adipocyte handling. In each of protocols some tissue was set aside for measuring fat cell size (61) and the remaining tissue was immediately rinsed with saline through Nitex Nylon Fiber 250/50. To measure the whole adipose tissue lipid SA the freshly rinsed tissue was added to $15 \mathrm{ml} \mathrm{CHCl3}$ : methanol (2:1) and incubated in a cold room for at least 2 days. Subsequently, $3.75 \mathrm{ml} 88 \% \mathrm{KCL}$ was added. The lipid layer was isolated by centrifugation, dried and weighed. The ${ }^{14} \mathrm{C}$ adipose lipid SA (dpm/g) from each biopsy was measured to less than $2 \%$ counting error as previously described (56).

To specifically measure adipocyte lipid SA in protocol 1 we isolated fat cells by digesting adipose tissue with $1 \mathrm{mg} / \mathrm{ml}$ collagenase type II (Sigma, St. Louis, MO) in HEPES buffer $(0.1$ M HEPES, $0.12 \mathrm{M} \mathrm{NaCl}, 0.05 \mathrm{M} \mathrm{KCl}, 0.005 \mathrm{M}$ glucose, $1.5 \%$ w/v BSA, $1 \mathrm{mM} \mathrm{CaCl} 2.2 \mathrm{H}_{2} \mathrm{O}$, $\mathrm{pH}$ 7.4) for $20 \mathrm{~min}$ at $37^{\circ} \mathrm{C}$. This cell suspension was centrifuged at $300 \times \mathrm{g}$. Adipocytes were obtained from the resulting floating layer and their lipids were extracted as described above for the whole adipose tissue using $\mathrm{CHCl}_{3}: \mathrm{MeOH}(2: 1)$ and KCL. We applied a second wash with HEPES buffer and centrifugation of the adipocyte layer before lipid extraction to ensure the elimination of extracellular FFA from the adipocyte layer.

Determining tracer distribution within adipose biopsies. 100-400 mg of lipid extract from each adipose sample was dissolved in chloroform and applied onto 1-4 thin layer chromatography (TLC) plates (silica gel $60 \AA$, size $20 \times 20 \mathrm{~cm}$, layer thickness $500 \mu \mathrm{m}$ ) (Whatman Inc., Clifton, NJ, Catalogue No. 4861-830). The lipid mass applied allowed us to obtain $<2 \%$ counting error with $\leq 100$ min of counting. The lipid classes on TLC plates were identified using standards and each class was collected by scraping, then re-extracted using hexaneschloroform-diethyl ether, 5:2:1 (v/v/v) and counted for radioactivity. The percent contribution of each lipid class to the total number of counts was calculated.

Measuring $m R N A$ for fatty acid transporters. Subcutaneous adipose tissue from abdomen and thigh was washed and flash frozen until extraction. RNA was extracted using RNeasy lipid tissue mini kit (Qiagen, Valencia, CA). A cDNA was made using a high capacity cDNA archive kit (Applied Biosystems, Foster City, CA). Quantitative RT-PCR was performed on an ABI 7900 using primer and probe sets from Applied Biosystems. Calculations of relative transcript amounts were normalized to a "housekeeping"/endogenous control gene (cyclophilin A) and then reported relative to a calibrator sample (surgical fat) using the DDCt method described in Applied Biosystem's Sequence Detection System User Bulletin \#2 (2001) "Relative quantitation of gene expression. (http.docs.applied biosystems. com/pebiodocs/04303859.pdf).

\section{Calculations}

Visceral fat mass was predicted using the CT measures of intra-abdominal and subcutaneous adipose tissue combined with DEXA measured abdominal fat (25). Upper body subcutaneous 
fat was calculated by subtracting visceral fat from total upper body fat measured by DEXA. Leg fat was measured using the region of DEXA interest program.

The uptake of FFA tracer into adipose tissue was calculated by multiplying the adipose tissue TG SA (dpm/gm) by the site-specific (lower body and upper body subcutaneous) adipose TG mass (gm). The ${ }^{14} \mathrm{C}$ FFA uptake is presented both as an absolute value (dpm/gm of tissue) and as a percentage of the total tracer infused. The later was calculated by dividing the regional adipose tracer uptake $(\mathrm{dpm})$ by the amount of tracer administered.

Insulin sensitivity was estimated using the HOMA 2 model (HOMA 2 IR) using an Excel macro courtesy of University of Oxford, England, 2004.

\section{Statistics}

All data are presented as mean \pm SEM unless otherwise stated. Statistical analyses were performed by t-tests using ANOVA with a repeated measures for upper and lower body fat within the same individuals, followed up by paired t-tests as needed. Comparisons between men and women were done using a non-paired t-test. $\mathrm{P}$ values $<0.05$ were considered statistically significant.

\section{Results}

\section{Protocol 1. Distribution of FFA tracer in adipose tissue lipid fractions}

Data from volunteers in protocols 1 and 2 were used to define the disappearance of the FFA tracer from plasma and the appearance of tracer in circulating TG (Figure 2.3). Following the bolus administration of the FFA tracer, plasma FFA SA was maximum at 1 min and decreased to $8 \%$ and $3 \%$ of maximum by $15 \mathrm{~min}$ and $30 \mathrm{~min}$, respectively. No sex differences were observed in the SA decay curves.

Figure 2.3. Kinetics of radiolabeled FFA in plasma and plasma TG. The decay of plasma FFA radioactivity (solid lines) and appearance in plasma triglycerides (dashed lines) before and after the IV bolus administration of $\left[{ }^{14} \mathrm{C}\right]$ FFA. Open circles represent values for women; closed circles represent values for men. In Protocol 2, adipose tissue biopsies were collected 40-45 min after the bolus, a time when most of the tracer has disappeared from plasma but has not substantially entered circulating triglyceride

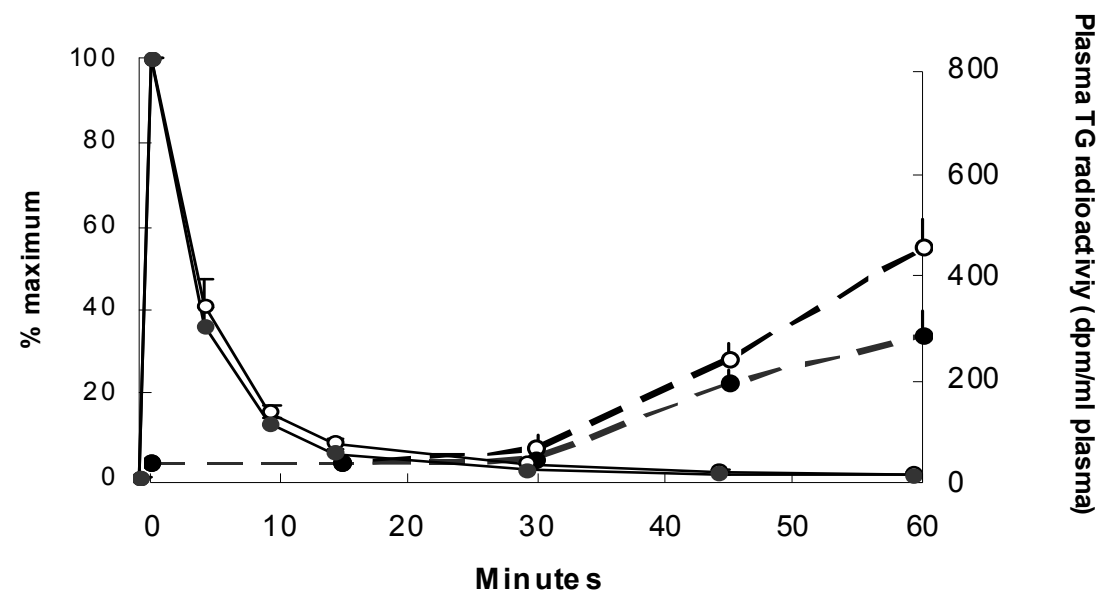


In contrast, lipid extracts from whole adipose tissue sometimes had a significant portion of the radioactivity in the FFA fraction, although $>95 \%$ of radioactivity was in the FFA and triglyceride fractions combined. Biopsies collected earlier in relation to the tracer bolus had a greater proportion of the radioactivity in the FFA fraction; as much as $60 \%$ at $15 \mathrm{~min}$ after the tracer bolus (Figure 2.4).

In adipose tissue samples collected $30 \mathrm{~min}$ after the tracer bolus an average of $25 \%$ of the radioactivity was in the FFA fraction in men whereas only $4 \%$ of the counts were in this fraction in women (Figure 2.4). In men, there was a positive relationship between the concentration of FFA tracer in plasma $(\mathrm{dpm} / \mathrm{ml})$ at the time of biopsy and the concentration of radioactivity in the fatty acid fraction of whole adipose tissue (Figure 2.4, inset), suggesting the tissue extract included extracellular fluid which contained plasma FFA tracer.

Figure 2.4. 14C palmitate in the FFA fraction of whole adipose tissue lipid. Percent of ${ }^{14} \mathrm{C}$ in lipid extract of whole adipose tissue that was found in the FFA fraction in abdominal and femoral subcutaneous depots in women (right) and men (left) at $15 \mathrm{~min}$ and $30 \mathrm{~min}$ after the IV bolus administration of $\left[1-{ }^{14} \mathrm{C}\right]$ palmitate. Inset relationship between plasma FFA radioactivity at the time of the biopsy (x-axis) and the radioactivity in the fatty acid fraction of whole adipose tissue measured using TLC (y-axis) in men.

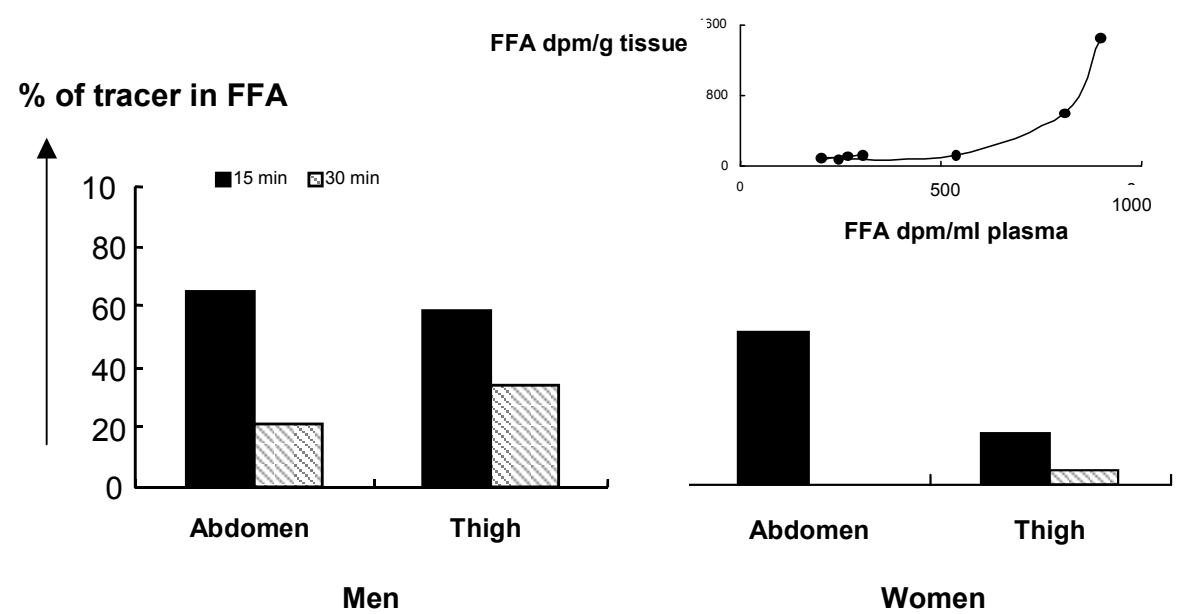

We could not confirm a similar relationship between plasma FFA radioactivity and adipose FFA radioactivity in women; biopsies taken $30 \mathrm{~min}$ after the bolus had a very small proportion of tracer in FFA regardless of the level of residual radioactivity in plasma.

From these studies we concluded that: 1) adipocytes take up FFA directly from the circulation; 2) the FFA taken up by adipocytes are for all practical purposes solely found in TG; 3 ) whole adipose tissue lipid extracts from biopsies taken $\leq 30$ min after an FFA tracer bolus may contain extracellular FFA that is present in plasma; 4) this problem can be overcome by isolating adipocytes or by delaying the biopsy until there is virtually no tracer remaining in plasma. 
Protocol 2: Subject characteristics (Table 2.2.1)

The lean men were slightly older than the lean women and the BMI was greater in men than in women. Consistent with previous results, women had a greater percent body fat, more leg fat and less visceral fat; abdominal and thigh fat cell size was not significantly different in men and women. Fasting plasma glucose, FFA and insulin concentrations in men and women were $5.2 \pm 0.1$ vs. $4.9 \pm 0.1 \mathrm{mmol} / \mathrm{L}, 463 \pm 35$ vs. $415 \pm 24 \mu \mathrm{mol} / 1$ and $33 \pm 4$ vs. $27 \pm 3$ $\mathrm{pmol} / \mathrm{l}(\mathrm{all} \mathrm{P}=\mathrm{NS})$. The HOMA IR values for men and women both averaged $0.5 \pm 0.1$.

Abdominal adipose tissue blood flow was greater in men than women ( $4.5 \pm 0.7$ vs. $2.4 \pm 0.3$ $\mathrm{ml} \cdot 100 \mathrm{gm}^{-1} \cdot \mathrm{min}^{-1}$, respectively, $\left.\mathrm{P}=0.001\right)$, whereas femoral adipose tissue blood flow was not different $\left(2.7 \pm 0.5\right.$ vs. $2.7 \pm 0.3 \mathrm{ml} \cdot 100 \mathrm{gm}^{-1} \cdot \mathrm{min}^{-1}$, respectively, $\left.\mathrm{P}=\mathrm{NS}\right)$.

Table 2.2.1. Volunteer characteristics

\begin{tabular}{|c|c|c|c|c|c|c|}
\hline & Protocol 2 & & & Protocol 3 & & \\
\hline & women & men & $\mathbf{P}$ & women & men & $\mathbf{p}$ \\
\hline n & 12 & 15 & & 12 & 10 & \\
\hline Age (years) & $29 \pm 2$ & $35 \pm 3$ & 0.04 & $38 \pm 3$ & $38 \pm 2$ & 0.95 \\
\hline BMI $\left(\mathrm{kg} / \mathrm{m}^{2}\right)$ & $22.8 \pm 0.4$ & $23.9 \pm 0.4$ & 0.04 & $33.0 \pm 1.0$ & $33.4 \pm 0.9$ & 0.64 \\
\hline Fat $\%$ & $31 \pm 1$ & $20 \pm 2$ & $<0.0001$ & $46 \pm 2$ & $35 \pm 1$ & $<0.0001$ \\
\hline TBF (kg) & $18.3 \pm 0.7$ & $15.6 \pm 1.6$ & 0.11 & $40.8 \pm 2.4$ & $35.5 \pm 2.5$ & 0.14 \\
\hline Leg fat (kg) & $7.7 \pm 0.5$ & $5.4 \pm 0.4$ & 0.002 & $15.9 \pm 1.3$ & $11.5 \pm 0.9$ & 0.009 \\
\hline UBSQ fat (kg) & $10.4 \pm 0.6$ & $8.9 \pm 1.3$ & 0.24 & $21.0 \pm 1.2$ & $17.9 \pm 1.3$ & 0.10 \\
\hline VISC fat (kg) & $1.2 \pm 0.3$ & $1.0 \pm 0.3$ & 0.60 & $4.0 \pm 0.4$ & $6.1 \pm 0.7$ & 0.02 \\
\hline $\begin{array}{l}\text { Abd FCS ( } \mu \mathrm{g} \\
\text { lipid/cell) }\end{array}$ & $0.411 \pm 0.036$ & $0.443 \pm 0.049$ & 0.51 & $0.858 \pm 0.073$ & $0.750 \pm 0.048$ & 0.24 \\
\hline $\begin{array}{l}\text { Fem FSC }(\mu \mathrm{g} \\
\text { lipid/cell })\end{array}$ & $0.481 \pm 0.027$ & $0.411 \pm 0.029$ & 0.11 & $0.813 \pm 0.082$ & $0.801 \pm 0.071$ & 0.91 \\
\hline
\end{tabular}

Values are mean \pm standard error. $\mathrm{Abd}=$ abdominal; $\mathrm{Fem}=$ femoral; $\mathrm{TBF}=$ Total body fat $\mathrm{UBSQ}=$ upper body subcutaneous; VISC $=$ visceral; FCS $=$ fat cell size

\section{Protocol 2: FFA uptake in adipose tissue}

Because women and men received the same amount of tracer $(41 \pm 1$ and $43 \pm 1 \mu \mathrm{Ci}$, respectively, $\mathrm{P}=0.22)$, the concentration of tracer in adipose lipid $(\mathrm{dpm} / \mathrm{gm})$ can be used as a direct index of the efficiency of adipocyte FFA uptake between groups. In all cases the ${ }^{14} \mathrm{C} \mathrm{FFA}$ tracer concentration was $<100 \mathrm{dpm} / \mathrm{ml}$ plasma at the time of the biopsy. We readily detected ${ }^{14} \mathrm{C}$ in whole adipose tissue lipid from the biopsy taken $40-45$ minutes after the tracer bolus. The adipose lipid SA in abdominal fat was greater in women than in men (455 \pm 58 vs. $297 \pm$ $35 \mathrm{dpm} /$ gram, respectively, $\mathrm{P}=0.03$ ).

Likewise, the adipose lipid SA in femoral fat was also greater in women than in men (463 \pm 42 vs. $229 \pm 23 \mathrm{dpm} /$ gram, respectively, $\mathrm{P}<0.0001)$. The adipose lipid SA in abdominal fat was greater than femoral fat $(\mathrm{P}=0.048)$ in men, but not in women $(\mathrm{P}=0.72)$. To put it another way, the efficiency of FFA uptake (per gram of fat) was $\sim 30 \%$ greater in abdominal fat that femoral fat in men, but was not different in women.

The portion of the tracer stored in upper body subcutaneous and leg fat is depicted in the right panel of Figure 2.5; the fraction of the tracer taken up in subcutaneous adipose lipid was greater $(\mathrm{P}<0.0001)$ in women than in men $(8.2 \pm 0.6 \%$ vs. $4.0 \pm 0.5 \%$, respectively $)$. 
Figure 2.5. Sex and depot differences in adipose tissue FFA tracer uptake. The lipid specific activity of abdominal and femoral adipose tissue in non-obese men and women (left panel) and the percent of the tracer stored in upper body subcutaneous and lower body fat (right panel). In both depots, tracer uptake is greater in women than in men $* \mathrm{p}<0.05$, *** $\mathrm{p}<0.001$ men vs. women; $\S \mathrm{p}<0.05$ upper body subcutaneous (UBSQ) vs. leg fat.
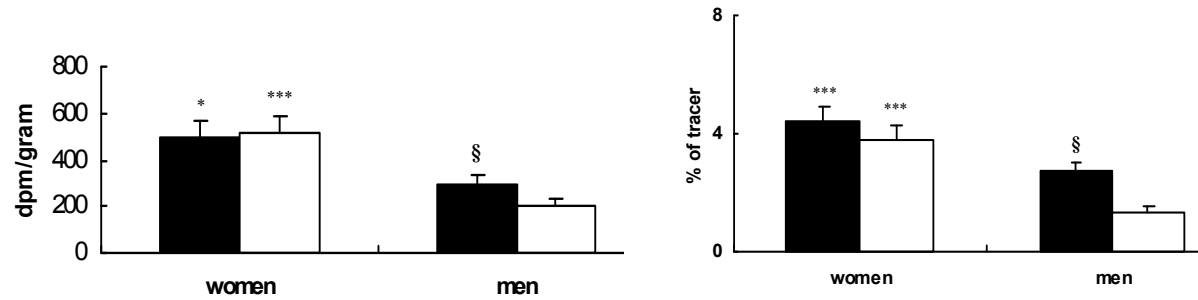

Protocol 3: Subject characteristics (Table 2.2 1).

The obese men and women were well matched for BMI. The waist-to-hip ratio (WHR) was $0.98 \pm 0.02$ for men and $0.89 \pm 0.01$ for women. The expected differences in percent body fat and regional fat mass were observed; abdominal and thigh fat cell size was not significantly different in men and women (Table 2.2.1). Fasting plasma glucose and insulin concentrations were similar in both groups: $5.3 \pm 0.1 \mathrm{mmol} / 1$ and $62 \pm 13 \mathrm{pmol} / 1$ in men and $5.2 \pm 0.1$ $\mathrm{mmol} / \mathrm{L}$ and $69 \pm 5 \mathrm{pmol} / \mathrm{l}$ in women. Fasting plasma FFA concentrations were higher in obese women than in men $(490 \pm 24 \mu \mathrm{mol} / 1$ vs. $384 \pm 37 \mu \mathrm{mol} / 1$ respectively $(\mathrm{p}=0.03)$. The HOMA IR values for men and women averaged $1.4 \pm 0.3$ and $1.1 \pm 0.1$, respectively.

\section{Protocol 3: FFA uptake in adipose tissue}

The women and men received $111 \pm 6$ and $123 \pm 8 \mu \mathrm{Ci}$ of ${ }^{14} \mathrm{C}$ palmitate, respectively $(\mathrm{P}=$ 0.25 , women vs. men). The ${ }^{14} \mathrm{C}$ whole adipose lipid SA from the tissue collected at the end of the tracer infusion are depicted in Figure 4. In obese women the adipose lipid SA was $\sim 40 \%$ greater in femoral than abdominal subcutaneous fat (1474 \pm 174 vs. $1048 \pm 105 \mathrm{dpm} / \mathrm{gram}$ fat, respectively, $\mathrm{P}=0.02)$. Abdominal adipose lipid $\mathrm{SA}$ tended to be greater than the femoral SA in obese men $(877 \pm 123$ vs. $721 \pm 98 \mathrm{dpm} / \mathrm{g}$, respectively, $\mathrm{P}=0.15)$. The femoral adipose tissue lipid SA was greater $(\mathrm{P}=0.002)$ in women than men, but the abdominal adipose SA was not statistically different $(\mathrm{P}=0.30)$.

We calculated that $11 \pm 3 \%$ of the total amount of tracer infused was taken up by lower body adipose tissue in obese women and only $3 \pm 1 \%$ was stored in lower body fat in obese men (P $=0.02$, women vs. men), reflecting both a greater efficiency of uptake and the greater leg fat mass in women (Table 1). The tracer uptake in upper body subcutaneous fat was somewhat greater in women than men $(9 \pm 1 \%$ vs. $7 \pm 2 \%, \mathrm{P}=0.15)$.

\section{Gene expression of fatty acid transport proteins in adipose tissue}

Abdomen and thigh adipose tissue from 12 normal weight women and 7 normal weight men (6 women and 3 men who participated in Protocol 2) was analyzed for mRNA abundance for CD36, FATP1 and FATP4. The transcript levels for the 3 fatty acid transporters relative to a calibrator sample are provided in Table 2.2.2. 
Figure 2.6. Sex and depot-specific tracer uptake in adipose tissue of obese men and women after continuous tracer infusion. Specific activity of abdominal and femoral fat depots in obese men and women (top panel) and the percent of the tracer stored in upper body subcutaneous and lower body fat (lower panel). $\S \mathrm{p}<0.05$ upper body subcutaneous (UBSQ) vs. leg fat; $* * * \mathrm{p}<$ 0.001 men vs. women
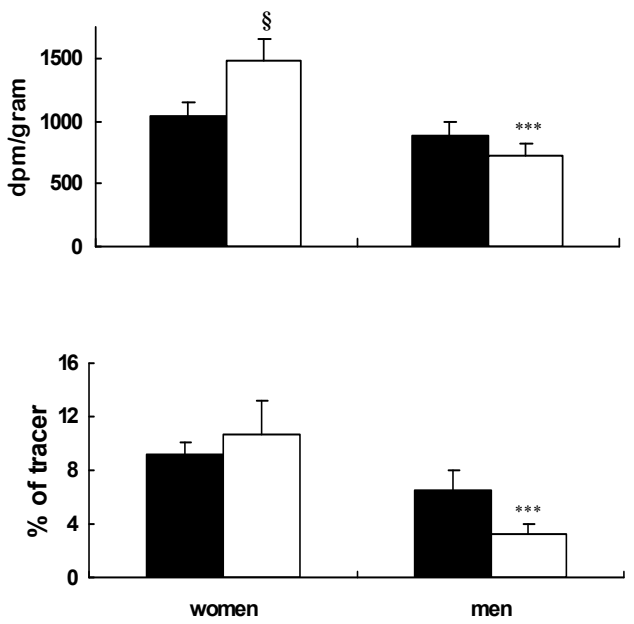

We tested the hypothesis that regional differences in the messages for the fatty acids transporters would follow the same pattern as the regional differences in direct FFA uptake.

Consistent with this hypothesis, the gene expression of the three fatty acid transporters was greater $(\mathrm{P}=0.04)$ in abdominal adipose than femoral adipose tissue in men, whereas they were not different $(\mathrm{P}$ $=0.80$ ) between abdomen and thigh in women. The gene expression levels of CD36 and FATP4 were greater in the thigh adipose of women than of men (both $\mathrm{P}=0.02$ ).

\section{Discussion}

Neither regional differences in lipolysis $(42,43-45,46,47,63)$ nor regional differences in adipose tissue uptake of mealderived fatty acids $(49,50,52-54)$ can explain how differences in body fat distribution are maintained between men and women. Herein we report that adipocytes directly take up circulating FFA in the postabsorptive state and that differences in FFA uptake efficiency are related to body fat distribution. A significant fraction of FFA disposal is direct adipocyte FFA uptake and there are important differences between lean and obese men and women. Direct FFA uptake in subcutaneous fat differs from meal fatty acid uptake in two respects: 1) direct FFA uptake is more efficient in women than men; 2) there is no preferential direct FFA uptake in upper body subcutaneous fat compared with lower body fat in women. These sex-based differences are consistent with this process as a mechanism to develop or maintain variations in body fat distribution between men and women, both lean and obese.

Table 2.2.2 Fatty acid transporter gene expression

\begin{tabular}{lllll}
\hline & \multicolumn{2}{c}{ Women } & \multicolumn{2}{c}{ Men } \\
\hline & Abdomen & Thigh & Abdomen & Thigh \\
CD36 & $2.51 \pm 0.51$ & $2.74 \pm 0.64 \dagger$ & $2.42 \pm 0.48$ & $2.17 \pm 0.35$ \\
FATP1 & $1.85 \pm 0.54$ & $1.71 \pm 0.46$ & $2.12 \pm 0.42$ & $1.74 \pm 0.36$ \\
FATP4 & $1.48 \pm 0.56$ & $1.55 \pm 0.30 \dagger$ & $1.40 \pm 0.29$ & $1.26 \pm 0.20$ \\
Average & $2.51 \pm 0.51$ & $2.00 \pm 0.42$ & $1.98 \pm 0.26$ & $1.72 \pm 0.24 *$ \\
\hline
\end{tabular}

Values are mean \pm one standard deviation for the gene expression relative to a calibrator sample. Abdominal and thigh samples from 12 normal weigh women and 7 normal weight men were analyzed. $* \mathrm{P}<0.05$ vs. abdomen; $\dagger \mathrm{P}<0.05$ vs. thigh for men

Virtually all of the FFA tracer recovered in lipid extracts from isolated adipocytes was in TG, whereas significant amounts of radioactivity were found in the FFA fraction of whole adipose tissue lipid, unless the biopsy was performed 40 - 45 min after the FFA tracer injection. The radioactivity in the FFA fraction of whole adipose tissue lipids collected at earlier time points can be explained by the residual radioactivity in the extracellular fluid that inevitably accom- 
panies adipose tissue. Although direct FFA uptake was detected in biopsies done $\leq 30 \mathrm{~min}$ following tracer injection, this required isolation of adipocytes from several hundred $\mathrm{mg}$ of adipose tissue or the isolation of TG from the whole adipose tissue lipid. A much simpler approach was to wait until 40-45 min after the tracer bolus to collect the tissue samples.

By administering the same dose of FFA tracer to normal weight men and women we could use the concentration of ${ }^{14} \mathrm{C}$ in adipose lipid as a direct measure of FFA uptake efficiency. Direct FFA uptake in subcutaneous adipose tissue was $\sim 70 \%$ more efficient in women than that in men (Protocol 2). Fat cell size was not different between men and women and abdominal adipose blood flow was greater in men than in women, which would be expected to favor greater, not lesser direct FFA uptake in men.

Edens et al (61) reported that subcutaneous adipose tissue fragments from women took up and esterified extracellular radiolabeled FFA twice as well as subcutaneous fat tissue from men. This suggests that adipocyte properties, rather than external factors, account for the greater FFA uptake in women in vivo. Because passive transmembrane transport of FFA (the so-called "flip-flop" mechanism) seemed unlikely to explain our findings, we looked for evidence for regional difference in facilitated transport. Thigh adipose tissue expressed the mRNA coding for fatty acid transport proteins more so in women than men and, on average, there was greater mRNA expression for transport proteins in abdominal than femoral subcutaneous fat in men. To the extent that message levels relate to protein content/function, the greater direct FFA uptake in abdominal than femoral fat in men could be due to greater facilitated inward fatty acid transport.

We tested whether total recycling of tracer from FFA back into adipocytes (both direct FFA uptake and any contribution from VLDL-TG uptake) maintained this sex specific pattern in obese men and women by performing fat biopsies following a continuous ${ }^{14} \mathrm{C}$ palmitate infusion. The efficiency of FFA storage in adipose TG was greater in obese women than obese men and women showed a preference for femoral adipose uptake. A portion of the FFA tracer could have entered adipose via VLDL-TG, although the relatively slow turnover of VLDLTG compared with FFA (62) makes it unlikely this pathway contributed substantially. Irrespective of the relative contribution of direct uptake vs. VLDL-TG uptake into subcutaneous adipose tissue in obese women, the pattern supports a preferential lower body fat distribution.

The implications of our findings for body fat distribution relates to the discrepancy between regional lipolysis and regional direct FFA uptake into adipocytes. Upper body subcutaneous fat contributes $\sim 70 \%$ of systemic FFA in lean men and women, whereas the leg contributes only about $\sim 20 \%$ (47); meal fatty acid uptake follows a similar pattern (52). In nonobese men the direct uptake/storage of FFA in abdominal and leg fat mirrors this regional difference in FFA release, whereas in lean women direct FFA uptake was similar in abdominal and femoral adipose tissue. This imbalance between release and direct reuptake in women could redistribute fatty acids towards leg fat. In the postabsorptive state, the systemic FFA flux in men and women averages $\sim 500 \mu \mathrm{mol} / \mathrm{min}(\sim 8.9 \mathrm{gm} / \mathrm{hr}$ or $107 \mathrm{gm}$ during a $12 \mathrm{hr}$ postabsorptive state)(47,57), of which $8.2 \%$ and $4.0 \%$ would be recycled back to subcutaneous adipose tissue via direct uptake in lean women and men, respectively. If the $\sim 8 \%$ of direct FFA uptake in subcutaneous fat in women were in proportion to rates of regional FFA release, 6.8 and 1.9 gm of FFA should be recycled into upper and lower body fat, respectively. Instead, we calculate that 4.7 and 4.1 of FFA would be directly taken up into upper and lower body subcutaneous fat in non-obese women.

Thus, in postabsorptive women, the direct adipose FFA uptake pathway could result in the redistribution of $\sim 2.1 \mathrm{gm} /$ day (779 gm/year) of fatty acids from upper body to lower body fat compared with what would occur if direct reuptake occurred in proportion to release. Us- 
ing the same approach we estimate that $\sim 0.5 \mathrm{gm} /$ day ( $\mathrm{P}=\mathrm{NS}$ vs. $0 \mathrm{gm} /$ day) of lipid would be redistributed from upper to lower body fat in lean men. In obese women the total FFA reuptake in leg fat was also significantly greater than in abdominal fat.

One might question how adipocytes, which are actively exporting FFA in the postabsorptive state, can simultaneously take up FFA. Because in these studies the tracer is administered intravenously it must mix in the central venous circulation with FFA released from (mostly) adipose tissue, enter the arterial circulation and be then be taken up as blood perfuses adipose tissue. Given that $\sim 4 \%$ (men) and $\sim 8 \%$ (women) of FFA are directly taken up in subcutaneous fat, and that subcutaneous fat accounts for $\sim 85 \%$ of systemic FFA flux (splanchnic release accounting for the other $15 \%$ (47)), we calculate that $1 / 20$ vs. $1 / 10$ FFA that are released by subcutaneous fat cells are subsequently taken up directly and independent of the LPL pathway.

This suggests a form of partitioning between adipocyte FFA release and FFA uptake. The partitioning could be at the level of each adipocyte, with the arterial-capillary delivery being somewhat anatomically distinct from the capillary-venous effluent. Alternatively, it may be that some populations of fat cells, such as the smaller adipocytes, take up but do not as actively release FFA, whereas larger fat cells briskly release FFA and do not take up FFA under postabsorptive circumstances.

Finally, it is possible that there are micro-collections of adipocytes that are undergo cyclic, temporarily inhibition of lipolysis that allows them to shift to fatty acid uptake. This shift to direct FFA uptake has been reported to occur on a larger scale in the postprandial situation (Bickerton et al, Diabetes 56:168-176, 2007), when suppression of lipolysis is accompanied by direct FFA uptake. In addition, in the postprandial state the substantial inflow of chylomicron derived fatty acids into adipose tissue, which still remains the major source of adipocyte TG fatty acids, could "carry" FFA along the same pathway.

A limitation of these studies is that to estimate total, direct FFA uptake we have extrapolated the regional tracer uptake from anterior abdominal subcutaneous adipose tissue to all upper body subcutaneous adipose tissue. Although this seems reasonable based upon the limited heterogeneity of meal-derived fatty acid uptake (52), further studies are needed to be confident that extrapolation is reasonable. In addition, we have no data regarding omental or mesenteric adipose tissue direct FFA uptake, which would be an important next step to understand the relevance of this mechanism.

In summary, we report an unappreciated mechanism for adipocyte fatty acid uptake and storage that is independent of LPL and not thought to exist in the postabsorptive state. A novel aspect of this pathway is the greater efficiency in direct FFA uptake in subcutaneous adipose tissue of women compared with men. Of equal interest is that the direct uptake pathway does not favor upper body subcutaneous fat in women the way it does in men. The direct FFA uptake pathway can redistribute fat from upper body stores to lower body stores in women, but not in men. 
Chapter 2 


\title{
2.3 Basal and Insulin Regulated Free Fatty Acid and Glucose Metabolism in Humans
}

\author{
Samyah Shadid ${ }^{1}$, Jill A. Kanaley, Michael T. Sheehan, Michael D. Jensen \\ Am J Physiol Endocrinol Metab. 2007 Feb 13; [Epub ahead of print] \\ Endocrine Research Unit, Mayo Clinic, Rochester, Minnesota, USA
}

\begin{abstract}
These studies were done to examine the effects of body composition, resting energy expenditure (REE), sex and fitness on basal and insulin regulated FFA and glucose metabolism.

We performed 137 experiments in 101 non-diabetic, pre-menopausal women and men, ranging from low normal weight to Class III obese (BMI 18.0-40.5 kg/m²). Glucose flux was measured using $\left[6-{ }^{2} \mathrm{H}_{2}\right]$ glucose and FFA kinetics with $\left[9,10-{ }^{3} \mathrm{H}\right]$ oleate under either basal $(74$ experiments) or euglycemic, hyperinsulinemic ( $1.0 \mathrm{mU} / \mathrm{kg} \mathrm{FFM} / \mathrm{min})$ clamp conditions $(63$ experiments).

Results: Consistent with our previous findings, REE and sex independently predicted basal FFA flux, whereas fat free mass was the best predictor of basal glucose flux; in addition, percent body fat was independently and positively associated with basal glucose flux (total $\left.\mathrm{R}^{2}=0.52, \mathrm{P}<0.0001\right)$. Insulin suppressed lipolysis remained significantly associated with $\operatorname{REE}(\mathrm{r}=0.25, \mathrm{P}<0.05)$, but percent body fat also contributed (total adjusted $\mathrm{R}^{2}=0.36$ $\mathrm{p}<0.0001$ ), whereas sex was not significantly related to insulin-suppressed FFA flux. Glucose disposal during hyperinsulinemia was independently associated with peak $\mathrm{VO}_{2}$, percent body fat and FFA concentrations (total $\mathrm{R}^{2}=0.63, \mathrm{P}<0.0001$ ), but not with sex.

We conclude that basal glucose production is independently related to both FFM and body fatness. In addition, hyperinsulinemia obscures the sex differences in FFA release relative to REE, but brings out the effects of fatness on lipolysis.
\end{abstract}

\footnotetext{
${ }^{1}$ Current addresses: SS:VieCuri medical Center, Venlo, The Netherlands, JK: Department of Exercise Science, Syracuse University, Syracuse, NY 13244, MS:Department of Endocrinology, Marshfield Clinic - Weston Center, Weston, WI 54476
} 


\section{Introduction}

Abnormalities of adipose tissue free fatty acid (FFA) release have been directly and causally proven to mediate some of the metabolic abnormalities seen in obesity (65). The increasing prevalence of obesity and its detrimental influence on health requires that we better understand adipose tissue metabolism as it relates to FFA release. We have previously examined the relationship between body composition, resting energy expenditure (REE) and basal FFA as well as glucose production rates (66). We found that systemic FFA flux was related to REE, but not to fat free mass (FFM), whereas basal glucose production rates were related to FFM, but not REE (66). In addition, FFA flux was $\sim 40 \%$ greater in women than men at comparable FFA concentrations, whereas glucose flux was not different between men and women relative to FFM.

Unfortunately, we do not have a similar level of understanding as to the major factors relating to adipose tissue FFA release during hyperinsulinemia. It has been reported that glucose disposal during hyperinsulinemia is related to FFM (67), indices of fatness $(67,68)$, plasma FFA concentrations (69) and fitness (70), but we could not find evidence that similar influences on insulin-suppressed FFA release have been studied. We previously reported that lipolysis is less readily suppressed by hyperinsulinemia in upper body obese women than in non-obese or lower body obese women (43), and that obese men are resistant to insulin with respect to the suppression of lipolysis (71). In those studies we did not include enough participants in those studies to test the effects of sex, fitness and fat distribution vs. fatness on lipolysis.

To address this limitation we studied a large number of normal weight and obese volunteers under overnight postabsorptive and/or insulin clamp conditions. We studied plasma insulin concentrations routinely seen after meal ingestion in order to be able to relate the findings to postprandial regulation of lipolysis. Consistent with our previous findings, women had greater rates of basal lipolysis than men relative to REE (66). We also found that the factors known to affect glucose disposal during hyperinsulinemia from other studies were important correlates in our population. To our surprise, we found that body fatness was positively related to basal endogenous glucose production rates. We also found that moderate hyperinsulinemia abolishes the sex difference in FFA metabolism.

\section{Methods}

\section{Subjects}

These studies were approved by the Mayo Clinic IRB. Written, informed consent was obtained from 101 non-diabetic, pre-menopausal women and men, ranging from low normal weight (BMI $18 \mathrm{~kg} / \mathrm{m}^{2}$ ) to Class III obese (BMI $40.5 \mathrm{~kg} / \mathrm{m}^{2}$ ). The activity levels of the volunteers ranged from sedentary (no regular, vigorous activity) to almost daily, vigorous exercise. Thirty-six of the participants were studied under both euglycemic, hyperinsulinemic clamp conditions and under saline control, leading to 137 different total experiments. The subjects were weight stable at least 3 months before entering the research protocols, which were designed primarily to study intramuscular triglyceride metabolism (data not yet published). The volunteers underwent muscle biopsies as part of these studies, however the analysis of the muscle samples is not yet complete. The FFA flux and related data for 20 of the obese men and 19 of the women presented herein has been previously published as the pre-intervention data for a study comparing diet/exercise to pioglitazone (Chapter 4.5.3). The characteristics of the participants are given in Table 2.3.1. 


\section{Study Protocol}

Assessment of body composition was done with dual energy x-ray absorptiometry (DEXA) and a single slice $\mathrm{CT}$ at the level of $\mathrm{L}_{2-3}$ within 2 weeks of the studies using previously described approaches (60). On or about the same day, oxygen consumption $\left(\mathrm{O}_{2}\right.$ peak) and maximum heart rate were determined by a graded exercise test performed on a Quinton (Seattle, WA) motor driven treadmill using a modified Bruce protocol (72). Heart rate and rhythms were monitored continuously via a 10-lead ECG. The volunteers consumed an isocaloric diet for 5 days prior to the study (55\% carbohydrates, $30 \%$ fat and $15 \%$ protein), prepared by our General Clinical Research Center (GCRC). The evening of the last day the participants were admitted to the GCRC and remained fasted after the evening meal. Prior to beginning the tracer infusions, baseline blood samples were collected for measurement of background plasma FFA specific activity (SA) and glucose enrichment. An intravenous catheter was placed in a forearm vein and kept patent with a continuous infusion of $0.45 \% \mathrm{NaCl}$.

At $0600 \mathrm{hrs}$ a primed, continuous infusion of $\left[6-{ }^{2} \mathrm{H}_{2}\right]$ glucose $\left(0.06 \mathrm{mg} \bullet \mathrm{kgFFM}^{-1} \bullet \mathrm{min}^{-1}\right)$ was begun to trace glucose kinetics. For the study of basal FFA kinetics, a continuous infusion of $\left[9,10-{ }^{3} \mathrm{H}\right]$ oleate $(0.8 \mu \mathrm{Ci} / \mathrm{min})$ was started at least $30 \mathrm{~min}$ prior to the FFA turnover sampling interval, which took place at $10 \mathrm{~min}$ intervals between 0830 and $0900 \mathrm{hrs}$. Resting energy expenditure was measured beginning at $0830 \mathrm{hrs}$ using a DeltaTrac Metabolic Cart (Yorba Linda, CA). The volunteers remained in the GCRC for the following $24 \mathrm{hrs}$. The same protocol was employed for those undergoing the insulin clamp study, except that at $0800 \mathrm{hrs}$ a euglycemic, hyperinsulinemic $(1.0 \mathrm{mU} / \mathrm{kg} \mathrm{FFM} / \mathrm{min})$ clamp was started using $\left[6-{ }^{2} \mathrm{H}_{2}\right]$ glucoselabeled $50 \%$ dextrose and continued for 6 hours to assure steady state glucose disposal and indirect calorimetry results. Blood was sampled every $10 \mathrm{~min}$ in order to allow adjustment of the glucose infusion rate to maintain blood glucose concentrations stable. Between 1330 and $1400 \mathrm{hr}$ resting energy expenditure was measured and blood samples were collected at $10 \mathrm{~min}$ intervals for measuring glucose enrichment and FFA SA were collected.

\section{Assessment of lipolysis using oleate flux}

FFA flux was calculated as $\mu \mathrm{mol} / \mathrm{min}$ using steady state formulas because concentration and SA were stable over the sampling interval. Under steady state conditions the amount of FFA released (FFA Ra) from adipose tissue equals the amount taken up (FFA Rd). The average oleate SA and glucose enrichments were used to calculate steady state rates of appearance (Ra) and disappearance (Rd) of oleate and glucose. Oleate flux was converted to FFA flux using the relationship between plasma oleate concentrations and total FFA concentrations (measured by HPLC) for the basal and insulin clamp conditions.

\section{Assays}

Glucose: Beckman glucose analyzer (Beckman Instruments, Fullerton, CA, USA); Glucose enrichment: gas chromatography/mass spectrometry (73); insulin, C-peptide and growth hormone concentrations: chemiluminescent sandwich assays (Sanofi Diagnostics, Chaska, MN, USA); catecholamines: HPLC with electrochemical detection (74). Plasma oleate concentrations and specific activity (SA), as well as total FFA concentrations, were measured by HPLC (75). Plasma triglyceride concentrations were measured using a centrifugal auto-analyzer (58).

\section{Statistical analysis}

Values are expressed as mean \pm standard deviation (and range). Statistical analyses were done using JMP 5.1.1 statistical package (SAS Institute Inc, Cary, NC). Statistical comparison between men and women were performed using non-paired t-tests. Univariate regression analy- 
sis was performed to test for simple relationships (such as the relationship between FFA concentration and FFA flux), whereas multivariate regression analysis was used to test the relationship between basal and insulin regulated FFA and indirect calorimetry, fitness $\left(\mathrm{VO}_{2}\right.$ peak) or body composition variables. The same approach was used to test for relationships between glucose flux and the above-mentioned variables.

For the analysis of insulin stimulated glucose disposal we initially included visceral fat area in the analysis, and although it contributed significantly and produced a greater $\mathrm{R}^{2}$, issues of multicollinearity of the data caused us to drop visceral fat area from the final model. Likewise, because $\mathrm{VO}_{2}$ peak was a significant predictor of insulin stimulated glucose disposal and is expressed as $\mathrm{ml} / \mathrm{kg} \mathrm{FFM} / \mathrm{min}$, we did not include FFM independently in the model. . We did not test models that expressed glucose or FFA turnover as $\mu \mathrm{mol} \bullet \mathrm{kg} \mathrm{FFM}{ }^{-1} \bullet \mathrm{min}^{-1}$. This is because we previously found that, for both of these variables, the intercept of the relationship is not zero and the slope is not one (66), which would be a prerequisite to expressing these kinetic relationships in such a ratio-standard manner (77).

\section{Results}

Subject characteristics (Table 2.3.1)

The characteristics of the participants in the basal and insulin clamp studies are presented in Table 2.3.1. On average, men were 3-6 years younger than women. Parenthetically, age was included in all of the analyses of FFA and glucose metabolism and did not contribute significantly.

Table 2.3.1. Subject characteristics.

\begin{tabular}{|c|c|c|c|c|}
\hline & \multicolumn{2}{|c|}{ Basal } & \multicolumn{2}{|c|}{ Insulin Clamp } \\
\hline & $\begin{array}{l}\text { Women } \\
(n=40)\end{array}$ & $\begin{array}{l}\text { Men } \\
(n=34)\end{array}$ & $\begin{array}{l}\text { Women } \\
(n=35)\end{array}$ & $\begin{array}{l}\text { Men } \\
(n=28)\end{array}$ \\
\hline Age (years) & $\begin{array}{l}36 \pm 10 \\
(19-50)\end{array}$ & $\begin{array}{l}33 \pm 8 \\
(22-50)\end{array}$ & $\begin{array}{l}39 \pm 8 \\
(21-50)\end{array}$ & $\begin{array}{l}33 \pm 8 \\
(23-50) \dagger\end{array}$ \\
\hline BMI $\left(\mathrm{kg} / \mathrm{m}^{2}\right)$ & $\begin{array}{l}26.7 \pm 6.9 \\
(18.0-38.3)\end{array}$ & $\begin{array}{l}27.1 \pm 6.2 \\
(19.7-40.5)\end{array}$ & $\begin{array}{l}28.0 \pm 6.5 \\
(18.0-37.8)\end{array}$ & $\begin{array}{l}28.6 \pm 5.7 \\
(19.7-40.5)\end{array}$ \\
\hline Weight (kg) * & $\begin{array}{l}73.8 \pm 19.6 \\
(45.8-107.8)\end{array}$ & $\begin{array}{l}88.4 \pm 21.2 \\
(61.0-131.1)\end{array}$ & $\begin{array}{l}76.9 \pm 17.9 \\
(45.8-109.1)\end{array}$ & $\begin{array}{l}92.8 \pm 19.1 \\
(61.0-131.1)\end{array}$ \\
\hline FFM (kg) * & $\begin{array}{l}45.2 \pm 5.8 \\
(34.3-58.1)\end{array}$ & $\begin{array}{l}64.7 \pm 6.2 \\
(50.8-76.6)\end{array}$ & $\begin{array}{l}46.1 \pm 5.1 \\
(34.3-56.2)\end{array}$ & $\begin{array}{l}64.8 \pm 5.9 \\
(50.8-80.5)\end{array}$ \\
\hline$\%$ body fat * & $\begin{array}{l}36 \pm 11 \\
(21-56)\end{array}$ & $\begin{array}{l}23 \pm 12 \\
(8-41)\end{array}$ & $\begin{array}{l}38 \pm 10 \\
(21-54)\end{array}$ & $\begin{array}{l}27 \pm 11 \\
(7-42)\end{array}$ \\
\hline WHR* & $\begin{array}{l}0.85 \pm 0.09 \\
(0.65-1.01)\end{array}$ & $\begin{array}{l}0.91 \pm 0.07 \\
(0.75-1.05)\end{array}$ & $\begin{array}{l}0.87 \pm 0.07 \\
(0.73-1.01)\end{array}$ & $\begin{array}{l}0.94 \pm 0.08 \\
(0.08-1.06)\end{array}$ \\
\hline $\operatorname{VISC}\left(\mathrm{cm}^{2}\right) *$ & $\begin{array}{l}73 \pm 62 \\
(6-224)\end{array}$ & $\begin{array}{l}129 \pm 114 \\
(18-451)\end{array}$ & $\begin{array}{l}84 \pm 66 \\
(6-236)\end{array}$ & $\begin{array}{l}156 \pm 123 \\
(22-447)\end{array}$ \\
\hline $\operatorname{ASC}\left(\mathrm{cm}^{2}\right)$ & $\begin{array}{l}187 \pm 148 \\
(20-536)\end{array}$ & $\begin{array}{l}158 \pm 137 \\
(22-460)\end{array}$ & $\begin{array}{l}222 \pm 148 \\
(20-448)\end{array}$ & $\begin{array}{l}198 \pm 133 \\
(34-460)\end{array}$ \\
\hline Peak VO 2 (ml/ kg FFM/min) & $\begin{array}{l}49.3 \pm 9.9 \\
(35.6-70.5)\end{array}$ & $\begin{array}{l}52.2 \pm 10.3 \\
(34.5-71.6)\end{array}$ & $\begin{array}{l}48.8 \pm 9.8 \\
(35.6-70.5)\end{array}$ & $\begin{array}{l}52.0 \pm 9.9 \\
(34.5-71.6)\end{array}$ \\
\hline Glucose (mg/dl) & $\begin{array}{l}91 \pm 9 \\
(78-117)\end{array}$ & $\begin{array}{l}91 \pm 9 \\
(77-117)\end{array}$ & $\begin{array}{l}93 \pm 10 \\
(78-115)\end{array}$ & $\begin{array}{l}93 \pm 9 \\
(77-110)\end{array}$ \\
\hline
\end{tabular}

Values are mean \pm one SD and range. * indicates a difference $(\mathrm{P}<0.005)$ between men and women, but no difference between the basal and insulin clamp groups. $\dagger$ indicates a difference $(\mathrm{P}=0.005)$ between men and women. $\mathrm{ASC}=$ Abdominal subcutaneous fat; VISC $=$ Visceral Fat; WHR=Waist-hip ratio. 
The differences between men and women in body weight, fat free mass, percent body fat, WHR, and visceral fat were as expected on the basis of previous observations. The men and women were not different with respect to BMI, abdominal subcutaneous fat area, peak oxygen consumption and fasting plasma glucose concentrations, nor were there differences between the basal and insulin clamp groups.

\section{Overnight postabsorptive results}

Basal plasma insulin concentrations were not different between men and women. Plasma FFA concentrations were $17 \%$ lower $(\mathrm{P}=0.03)$ in men than women (Table 2.3.2). Plasma FFA flux $(\mu \mathrm{mol} / \mathrm{min})$ was also somewhat lower in men than women $(\mathrm{P}=0.12)$.

Glucose flux $(\mu \mathrm{mol} / \mathrm{min})$ and REE were significantly greater in men than women, and the basal respiratory exchange ratio (RER) was not different between men and women.

Basal plasma FFA concentrations were highly correlated with plasma FFA flux $(\mathrm{r}=0.51$, $\mathrm{p}<0.0001)$. Consistent with our previous observations (66) plasma FFA flux was related to both REE $(\mathrm{p}<0.0001)$ and sex $(\mathrm{p}<0.0001)$. This relationship is depicted in Figure 2.7a. Together, these two factors could account for $26 \%$ of the variance of basal plasma FFA flux. Other factors, including WHR, percent body fat, visceral fat area, abdominal subcutaneous fat area, FFM, and peak $\mathrm{VO}_{2}$ were not significant contributors to basal FFA flux after REE and sex were taken into account.

Figure 2.7 Relationship between Resting Energy Expenditure and Free Fatty Acid (FFA) Flux. In panel "a" overnight postabsorptive plasma FFA flux is plotted vs. resting energy expenditure for each man and woman participating in these studies. Panel "b" depicts insulin suppressed plasma FFA flux plotted vs. resting energy expenditure measure during the insulin clamp for each man and woman participating in these studies

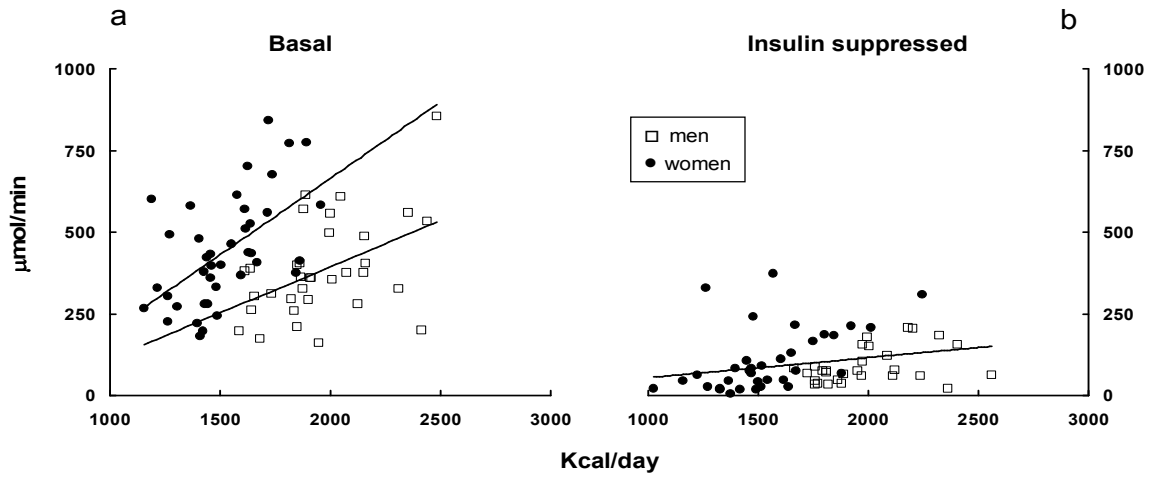

As we previously observed (66), basal glucose flux was significantly related to FFM (adjusted $\left.\mathrm{R}^{2}=0.38, \mathrm{P}<0.0001\right)$ and REE did not add significantly to predicting glucose flux. Interestingly, the combination of FFM and percent body fat substantially improved the ability to predict basal glucose flux (adjusted $\mathrm{R}^{2}=0.52, \mathrm{P}<0.0001$ ). The relationship between FFM and basal glucose flux residuals is depicted in Figure 2.8 and the relationship between basal glucose flux residuals and \% body fat is depicted in Figure 2.9.

None of the other variables, including basal plasma glucose or FFA concentrations, contributed significantly to the ability to predict basal glucose flux. The basal RER was not 
significantly related to any of the body composition variables, but we found that basal FFA concentrations were inversely correlated with basal RER values $(r=-0.28, P=0.007)$.

Figure 2.8 Overnight postabsorptive plasma glucose flux residuals vs. fat free mass (FFM)

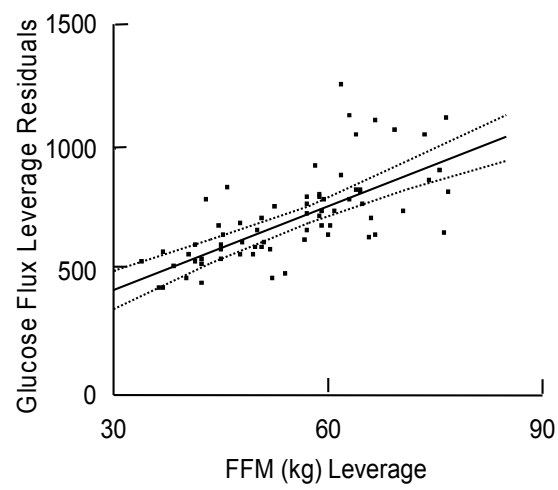

Figure 2.9 Overnight postabsorptive plasma glucose flux residuals vs. percent body fat.

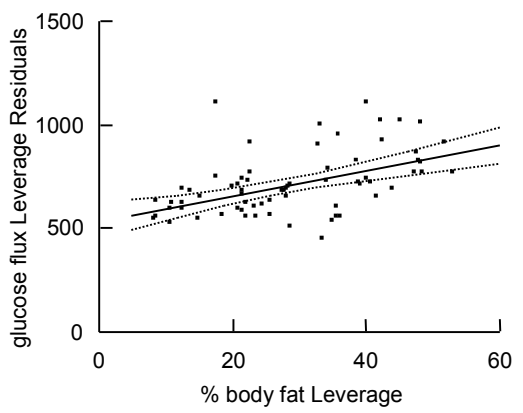

\section{Insulin clamp conditions}

The plasma insulin and FFA concentrations observed during insulin clamp in men and women are also provided in Table 2.3.2.

As expected, plasma FFA concentration and flux were suppressed as a result of hyperinsulinemia and plasma FFA concentrations and FFA flux were highly correlated with each other $(\mathrm{r}=0.68, \mathrm{p}<0.0001)$. Under the conditions of hyperinsulinemia the relationship between plasma FFA flux $(\mu \mathrm{mol} / \mathrm{min})$ and REE was maintained $(\mathrm{r}=0.22, \mathrm{P}<0.05$, Figure 1b), however, sex was no longer significantly related to FFA flux. The combination of REE and percent body fat predicted a significantly larger portion of the variance in insulin suppressed FFA flux (adjusted $\mathrm{R}^{2}=$ $0.36 \mathrm{p}<0.0001)$ than REE alone. None of the other variables significantly improved the ability to predict insulin suppressed FFA flux.

Glucose disposal ( $\mu \mathrm{mol} / \mathrm{min})$ values during the clamp are provided in Table 2.3.2. Although no one factor accounted for the majority of inter-individual variation in glucose disposal, a number of parameters combined could predict a large portion of the variance in glucose disposal (adjusted $\mathrm{R}^{2}=0.63, \mathrm{P}<$ $0.0001)$. These factors and the associated $\mathrm{P}$ values are provided in Table 2.3.3. Peak $\mathrm{VO}_{2}$ was positively related to total glucose disposal, whereas plasma FFA concentrations and percent body fat were independently and negatively related to glucose disposal.

The insulin clamp RER values were not dif-

ferent between men and women (Table 2.3.2) and were negatively and independently correlated with plasma FFA concentrations and visceral fat (both $\mathrm{P}=0.01$ ). Together, FFA and visceral fat could account for $25 \%$ of the variance in RER.

\section{Discussion}

Our group has previously examined the relationship between overnight postabsorptive FFA and glucose flux to understand the role of energy expenditure, sex and body composition in determining substrate metabolism (66). We found that FFA flux is more strongly related to REE than to body composition and that women have higher rates of lipolysis than men when 
adjusted for REE. This was not the case for basal glucose flux, which was similar in men and women and more strongly correlated with FFM than REE.

The goal of this study was to examine, in a larger cohort, the additional effects of fitness level on basal lipolysis and glucose flux, as well as to understand whether hyperinsulinemia disrupts these relationships. We confirmed that basal FFA flux is best related to REE and sex, but that during hyperinsulinemia body fatness rather than female sex is associated with greater FFA release independent of REE. To our surprise, both body fat and FFM were positively related to basal glucose flux, whereas a number of factors independently influenced insulin stimulated glucose disposal.

Table 2.3.2 Insulin, FFA, glucose and indirect calorimetry results.

\begin{tabular}{lllll}
\hline & Basal & & Insulin Clamp & \\
\hline & $\begin{array}{l}\text { Women } \\
(\mathbf{n}=\mathbf{4 0})\end{array}$ & $\begin{array}{l}\text { Men } \\
(\mathbf{n}=\mathbf{3 4})\end{array}$ & $\begin{array}{l}\text { Women } \\
(\mathbf{n}=\mathbf{3 5})\end{array}$ & $\begin{array}{l}\text { Men } \\
(\mathbf{n}=\mathbf{2 8})\end{array}$ \\
& & & \\
Insulin $(\boldsymbol{\mu} \mathbf{U} / \mathbf{m L})$ & $5.9 \pm 5.1$ & $5.9 \pm 5.8$ & $34.6 \pm 8.6$ & $45.9 \pm 12.8$ \\
& $(1.1-30.0)$ & $(0.7-26.1)$ & $(20.9-55.8)$ & $(27.3-76.8) \dagger$ \\
FFA $(\boldsymbol{\mu m o l} / \mathbf{L})$ & $455 \pm 127$ & $376 \pm 169$ & $74 \pm 82$ & $66 \pm 64$ \\
& $(183-653)$ & $(138-1042)^{*}$ & $(7-428)$ & $(20-323)$ \\
FFA flux $(\boldsymbol{\mu m o l} / \mathbf{m i n})$ & $442 \pm 67$ & $383 \pm 150$ & $108 \pm 98$ & $91 \pm 55$ \\
& $(181-843)$ & $(160-854)$ & $(5-372)$ & $(22-207)$ \\
Glucose flux $(\boldsymbol{\mu m o l} / \mathbf{m i n})$ & $638 \pm 155$ & $786 \pm 198$ & $1937 \pm 758$ & $2375 \pm 887$ \\
& $(396-1027)$ & $(492-1190) \dagger$ & $(602-4073)$ & $(1003-4226)$ \\
Study interval REE & $1524 \pm 200$ & $1961 \pm 243$ & $1541 \pm 250$ & $1992 \pm 231$ \\
(Kcal/day) & $(1155-1959)$ & $(1586-2482) \dagger$ & $(1028-2245)$ & $(1664-2562) \dagger$ \\
Study interval RER & $0.81 \pm 0.05$ & $0.81 \pm 0.03$ & $0.89 \pm 0.05$ & $0.87 \pm 0.07$ \\
& $(0.73-0.91)$ & $(0.74-0.90)$ & $(0.79-1.02)$ & $(0.73-1.03)$ \\
\hline
\end{tabular}

Values are mean \pm one SD and range. * indicates a difference $(\mathrm{P}<0.05)$ between men and women. $\dagger$ indicates a difference $(\mathrm{P}<0.005)$ between men and women. Comparisons between Basal and Insulin Clamp values are not made.

Consistent with our previous findings (66), basal FFA flux was not significantly related to body fat after adjusting for sex and REE. In this study we were also able to test whether fitness, as measured by peak $\mathrm{VO}_{2}$, is related to basal or insulin suppressed lipolysis. We found that peak $\mathrm{VO}_{2}$ was not related to FFA flux under either condition. This suggests that to the extent that physical fitness improves metabolic function, it does not do so via effects on basal or insulin suppressed lipolysis.

We also found that suppression of lipolysis by physiological insulin concentrations did not disrupt the relationship between FFA flux and REE. Of interest body fat, rather than sex, accounted for a significant portion of the intra-individual variability in FFA flux under insulin clamp conditions. We conclude that whatever factors mediate greater FFA availability in overnight postabsorptive women are readily overcome by moderate hyperinsulinemia. In addition, increasing body fat content is a significant modulator of lipolysis under insulinsuppressed conditions, independent of sex.

The finding that FFM is most strongly correlated with basal glucose flux $(66,67,76)$ does not inherently imply that dividing glucose flux rates by FFM is an optimal means of data expression (77). This is especially a problem when groups differ in FFM, because glucose flux and FFM are not in a ratio-standard relationship (a slope of one and an intercept of zero). A corollary to this issue is that it may be more difficult to detect the influence of other factors on glucose flux if a ratio-standard approach means of data expression is used. 
When we tested for the effects of fitness, body fat and sex on basal glucose turnover we were surprised to detect a significant, positive effect of percent body fat (Figure 2.9). Thus, even after adjusting for FFM, adults with more body fat have greater overnight postabsorptive glucose production/uptake rates at the same blood glucose concentration.

Table 2.3.3. Parameters relating to insulin stimulated glucose disposal.

\begin{tabular}{llll}
\hline Factor & Estimate & Standard Error & P value \\
\hline & & & \\
Intercept & 348 & 742 & 0.64 \\
Peak $\mathrm{VO}_{2}$ & 23.7 & 7.8 & 0.007 \\
Plasma FFA concentrations & -2.2 & 1.0 & 0.01 \\
Percent body fat & -17.5 & 8.5 & 0.02 \\
\hline
\end{tabular}

Insulin stimulated glucose disposal $(\mu \mathrm{mol} / \mathrm{min})$ as predicted by peak $\mathrm{VO}_{2}(\mathrm{ml} / \mathrm{kg} \mathrm{FFM} / \mathrm{min})$, plasma FFA $(\mu \mathrm{mol} / \mathrm{L})$ and percent body fat.

This effect was independent of plasma FFA concentrations. Although this has not been previously reported, we note that indices of fatness have been associated with basal gluconeogenesis, but not endogenous glucose production, in smaller groups of non-diabetic subjects $(67,76)$ and in T2DM (68). We speculate that by studying a much larger group of volunteers with a wide range of body fat and FFM we were able to detect this effect of fatness on endogenous glucose production.

An implication of this finding is that investigators may need to be cautious in comparing glucose flux, especially if expressed relative to FFM, amongst persons with widely different amounts of body fat. For example, leaner men and more overweight women could have similar amounts of FFM, but widely differing amounts of body fat. If basal endogenous glucose production were greater in the women this would not be proof of a sex effect per se, but rather an effect of greater body fat. For example, for the 7 men and 8 women in this study with FFM between $50-60 \mathrm{~kg}$ (average of 53 for women and 56 for men), glucose flux was $10.7 \pm 0.5$ and $13.7 \pm 0.9 \mu \mathrm{mol} \bullet \mathrm{kg} \mathrm{FFM}^{-1} \bullet \mathrm{min}^{-1}$, respectively $(\mathrm{P}=0.01$, men vs. women $)$, despite having the same mean fasting glucose concentrations ( $90 \mathrm{vs} .91 \mathrm{mg} / \mathrm{dL}$ ). From these studies it is not possible to determine whether the slightly greater production in those with more body fat is being driven by a factor produced by adipose tissue or whether greater glucose utilization is associated with greater amounts of adipose tissue. In theory the later would require greater glucose production to maintain plasma glucose concentrations.

The factors that we found to be predictive of insulin stimulated glucose disposal are those that would be predicted from the literature (45,78-80). Peak $\mathrm{O}_{2}$ was positively associated with glucose uptake, whereas percent body fat and insulin suppressed FFA concentrations were independently, negatively associated with glucose disposal. The amount of variance explained by these factors was quite high by most standards. We take this as evidence that our results are largely in keeping with the literature, which is reassuring given that some of our findings were unexpected.

The relationship between plasma FFA concentrations and RER deserves brief comment. It is difficult to attain true energy balance with 3 days of GCRC feeding, thus even though all volunteers received meals with the same food quotient, some probably were slightly underfed whereas others were slightly overfed. Because energy deficits are largely handled by mobilizing and oxidizing more FFA it is possible that the association we observed between postabsorptive RER and plasma FFA concentrations is an "energy deficit" effect. In contrast, it is 
doubtful that this same phenomenon would hold at the end of a 6 hour, euglycemic, hyperinsulinemic clamp. Thus, it may be that adipose tissue resistance to the ability of insulin to suppress lipolysis, resulting in greater FFA concentrations and greater fat oxidation, is the etiology of the association of the lower RER in those with greater plasma FFA.

Some limitations to this study must be acknowledged. In this study we had only a single measure of basal FFA flux and glucose flux, whereas we previously had quadruplicate measures of FFA flux and duplicate measures of glucose flux (66). Thus, although we have a larger group of participants, we do not have an equally precise measure of each individual's "true" average basal rate of lipolysis or glucose production. Because of this, we may have missed some associations that were not as strong as those with REE, sex, and body fat. Another limitation is that we cannot determine whether the increase in endogenous glucose production we observed as a function of body fat is related to effects on glycolysis or gluconeogenesis (76).

In summary, we report that FFA availability remains positively related to REE under conditions of moderate hyperinsulinemia, but that it is additionally affected by percent body fat. Fitness, sex and body fat distribution are not associated with insulin-suppressed lipolysis. We also found that percent body fat was significantly related to basal glucose turnover after adjusting for FFM. These new findings are in the context of data that confirm our previous work (66) regarding basal lipolysis and the work of others on insulin stimulated glucose disposal $(45,78-80)$. We conclude that differences in basal or insulin suppressed lipolysis are not associated with fitness and that investigators must take into account body fat when presenting data on basal glucose production between groups with differing amounts of FFM and/or body fat. 



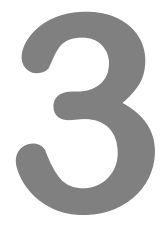

Obesity 
$\begin{array}{lll}3.1 & \text { Definitions and adverse effects of obesity } & 47\end{array}$

$\begin{array}{lll}3.1 .1 & \text { Definitions } & 47\end{array}$

3.1.2 Adverse Effects of obesity 47

3.1.2.1 The Metabolic Syndrome 48

3.1.2.2 Upper body obesity and insulin resistance: the portal theory 48

3.2 Pathophysiology: Effects of obesity on endocrine control of nutrient partitioning

From: 'Endocrine Control of Nutrient Partitioning'; Book chapter by S Shadid and MD Jensen for 'Obesity: Clnical Evaluation and Treatment', edited by Robert H. Eckel, MD. Lippincott, Williams and Wilkins, Feb 2003

3.2.1 Introduction $\quad 50$

3.2.2 General Considerations $\quad 51$

3.2.2.1 Processing of Fuel: Fat, Carbohydrate and Protein Metabolism 51

3.2.2.2 Energy Expenditure and Metabolism 53

3.2.3 Endocrine Control of Fuel Partitioning 54

3.2.3.1 Insulin $\quad 54$

A. Physiology 54

B. Insulin and Nutrient Partitioning in Obesity $\quad 57$

3.2.3.2 Growth Hormone $\quad 58$

A. Physiology $\quad 58$

B. Growth Hormone and Visceral Obesity 61

C. Growth Hormone Administration in Visceral Obesity 62

$\begin{array}{ll}\text { 3.2.3.3 Testosterone } & 62\end{array}$

A. Physiology 62

B. Testosterone and Visceral Obesity 64

C. Testosterone Administration in Visceral Obesity 64

$\begin{array}{ll}\text { 3.2.3.4 Estrogens } & 65\end{array}$

A. Physiology 65

B. Estrogen Replacement Therapy and Nutrient Partitioning 68

$\begin{array}{ll}\text { 3.2.3.5 Glucocorticoids } & 69 \\ \text { A. Physolog } & 69\end{array}$

A. Physiology $\quad 69$

B. The Hypothalamic-Pituitary-Adrenal Axis and Obesity 72

C. Managing Glucocorticoid Therapy in Obese Patients 73

$\begin{array}{ll}3.2 .4 \text { Conclusions } & 74\end{array}$ 


\subsection{Definitions And Adverse Effects Of Obesity}

\subsubsection{Definitions}

Obesity is a state of energy surplus of the body, arising when energy intake chronically and sufficiently exceeds energy expenditure (see Chapter 3.2.1). Its definitions are based on the Quetelet Index or body mass index (BMI), equaling the weight of an individual (kilograms) divided by the square of the height (meters).

BMIs between $18-25 \mathrm{~kg} / \mathrm{m}^{2}$ are considered normal, since BMIs outside this range are associated with increased health risks. Obesity is defined as a BMI $\geq 30$ and classified based on the WHO standard of 1997 (Table 3.1.1).

Table 3.1.1 Classification of obesity (World Health Organization)

\begin{tabular}{llll}
\hline BMI & Classification & \% Overweight & Health Risk \\
\hline$<18$ & Underweight & & moderate \\
$18-24.9$ & Healthy weight & & normal \\
$25-29.9$ & Overweight & $20-40$ & increased \\
$30-34.9$ & Obese Class I (mild) & $41-100$ & moderate \\
$35-39.9$ & Obese Class II (moderate) & $>100$ & severe \\
$>40$ & Obese Class III (severe) & & very severe \\
\hline
\end{tabular}

\subsubsection{Adverse Effects Of Obesity}

The adverse health effects of obesity are mainly metabolic and mechanical. In addition, obesity is associated with gallstones, gout, depression and several forms of cancer (breast, endometrium, prostate and pancreas) (81-84).

Mechanical complications include increased incidence of osteoarthritis, herniated disks, chronic low back pain and sleep apnea. Metabolic complications are mostly related to insulin resistance, and associated herewith, increased concentrations of free fatty acids (FFAs), triglycerides (TG) and glucose, and decreased HDL (high-density lipoprotein) cholesterol. This complex of factors has lead to the identification of the metabolic syndrome (see below) and may lead to diabetes mellitus, arteriosclerosis, and hence, additional (cardiovascular) morbidity and mortality. In the USA, $90 \%$ of type 2 diabetic patients are overweight (Harris in (85)).

Other metabolic/endocrine changes in obesity include those of growth hormone (86), cortisol (87-90) and testosterone (91,92) (Chapter 3.2.1), adipocytokines (Chapters 2.1.4 and 4.5.5) and the development of hepatic steatosis or non-alcoholic fatty liver disease (NAFLD; see Chapter 4.4.3). 


\subsubsection{The Metabolic Syndrome}

The term 'metabolic syndrome' was introduced to facilitate the identification of subjects at high risk for the development of cardiovascular disease and diabetes. Several similar, but not identical definitions have been formulated, among others by the World Health Organization (WHO), the European Group for the Study of Insulin Resistance (EGIR) and the U.S. National Cholesterol Education Program Adult Treatment Panel III (NCEP ATPIII) respectively (table 3.1.2). The NCEP definition is widely used in research; the WHO definition is slightly more complex and relies more on evidence of glucose deregulation.

The age-adjusted prevalence of the metabolic syndrome in the USA increased from $24 \%$ (NHANES 1994-1998) to 27\% (NHANES 1999-2000) (93). For comparison, this was 25 in Korean (94), $\sim 20 \%$ in Asian Indian adults (2006, depending on the criteria used (95), and $26 \%$ and $55 \%$ in elderly Italian men and women respectively (96).

Table 3.1.2 Definitions of the metabolic syndrome

\begin{tabular}{|c|c|c|}
\hline WHO 1999 & EGIR 1999 & NCEP 2001 \\
\hline $\begin{array}{l}\text { Diabetes or IGT or IR } \\
\text { PLUS } 2 \text { of the following: }\end{array}$ & $\begin{array}{l}\text { No Diabetes, but IR or hyperinsulinemia } \\
\text { PLUS } 2 \text { of the following }\end{array}$ & 3 of the following \\
\hline $\begin{array}{l}\text { 1.Dyslipidemia: } \\
\text { - } \mathrm{TG}>1.7 \mathrm{mmol} / 1 \text { and/or } \\
\text { - HDL }<0.9(\overbrace{}^{\lambda}),<1.0(+)\end{array}$ & 1. Fasting plasma glucose $>6.1 \mathrm{mmol} / \mathrm{l}$ & $\begin{array}{l}\text { 1. Fasting plasma glucose } \\
>6.1 \mathrm{mmol} / \mathrm{l}(>110 \mathrm{mg} / \mathrm{dl})\end{array}$ \\
\hline $\begin{array}{l}\text { 2.Hypertension: blood pressure } \\
>140 / 90 \mathrm{mmHg} \text { and/or medica- } \\
\text { tion }\end{array}$ & $\begin{array}{l}\text { 2. Dyslipidemia } \\
\text { - triglycerides }>2.0 \mathrm{mmol} / 1 \text { and/or } \\
\text { - HDL }<1.0 \text { or } \\
\text { - treated for dyslipidemia }\end{array}$ & $\begin{array}{l}\text { 2. Hypertriglyceridemia } \\
\text { triglycerides }>1.7 \mathrm{mmol} / 1\end{array}$ \\
\hline 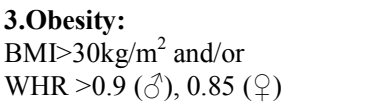 & $\begin{array}{l}\text { 3. Hypertension: blood pressure }>140 / 90 \\
\mathrm{mmHg} \text { and/or use of medication }\end{array}$ & $\begin{array}{l}\text { 3. Low HDL cholesterol } \\
<40 \text { in men, }<50 \mathrm{mg} / \mathrm{dl} \text { in } \\
\text { women }\end{array}$ \\
\hline \multirow[t]{2}{*}{4 Microalbuminuria: } & $\begin{array}{l}4 \text { Central obesity } \\
\text { Waist circumference }>94 \mathrm{~cm}(ð),>80 \mathrm{~cm}(\uparrow)\end{array}$ & $\begin{array}{l}\text { 4. Hypertension: blood pres- } \\
\text { sure }>130 / 85 \mathrm{mmHg} \text { and/or } \\
\text { medication }\end{array}$ \\
\hline & & $\begin{array}{l}\text { 5. Central obesity: Waist cir- } \\
\text { cumference }>102 \mathrm{~cm}(\AA) \text {, } \\
>88 \mathrm{~cm}(q)\end{array}$ \\
\hline
\end{tabular}

Source: ATP III Clinical Identification of the Metabolic Syndrome Circulation 2004; 109:433-438.) Abbreviations: BMI: body Mass Index; HDL: High Density Lipoprotein; IGT: impaired glucose tolerance; IR: insulin resistance; TG: triglycerides.

\subsubsection{Upper body obesity and insulin resistance: the portal theory}

Although the reciprocal interaction between insulin resistance and elevated FFA concentrations has long been recognized (97), the exact mechanism and its initiation remain unclear. One theory identifies the increased total fat mass in obesity per se as the initial culprit; this would lead to high total body lipolysis and thus to a high FFA load, secondarily triggering 
insulin resistance. This theory, however, cannot explain the metabolic difference between UBO and LBO: visceral fat mass is positively correlated with adverse health consequences and excess FFA availability, more so than subcutaneous and femoral fat. Nevertheless, visceral fat is not the source of excess systemic FFA availability: the largest part of circulating FFAs originates from truncal (upper body, non-visceral) fat, both in UBO and LBO humans, independently of fatness, gender and diabetic status $(43,44,47)$.

The metabolic difference between UBO and LBO has therefore been postulated to originate from regional differences in either adipocyte biology or FFA delivery. Older publications, indeed, indicated that omental (visceral) fat was substantially more resistant to insulin and insulin-mediated suppression of lipolysis than other fat depots $(98,99)$. However, recent data from healthy women have disputed this assertion, suggesting that visceral obesity has only minor effects on regional differences in adipose tissue metabolism (100): omental adipocytes were more responsive to adrenergic lipolytic agents than subcutaneous adipocytes, but the latter were larger and displayed both higher absolute lipolytic and relative LPL activity.

Another suggestion involves the anatomic relationship of visceral (omental and mesenteric) fat to the portal vein. FFAs released from visceral adipose tissue are directly delivered to the portal vein and may disproportionately affect the liver; this is thought to lead to hepatic insulin resistance $(98,101)$ and is generally known as the 'portal theory'. Increasing amount of visceral fat probably result in greater hepatic FFA delivery.

Indeed, overfeeding experiments in dogs resulted in rapid increases of visceral fat paralleling hepatic and peripheral insulin resistance (102); moreover, weight loss and the concomitant increase in insulin sensitivity is associated with a disproportionate loss of visceral fat compared to other adipose depots (Chapter 4.5.6). Nevertheless, evidence for an increased overall FFA flux into the liver in UBO has never been found. Neither is it clear why increased FFA flux would directly result in hepatic insulin resistance, or why increased FFA delivery to the liver should lead to increased peripheral insulin resistance, as opposed to an increased FFA flux originating from other fat depots. Indeed, a transgenic rat model of late-onset visceral obesity without insulin resistance has been presented recently (103).

In summary, increased plasma FFA concentrations play an important role in the pathophysiology of insulin resistance, but it remains unknown to what extent their quantity versus their kinetics are crucial in starting or maintaining the vicious circle of this reciprocal relationship. $\mathrm{UBO}$ is more frequently and more severely related to insulin resistance than LBO; the visceral fat compartment plays an important role herein but is likely to be part of a multifactorial process. 


\title{
3.2 Pathophysiology: Effects Of Obesity On Endocrine Control Of Nutrient Partitioning
}

\author{
Slightly modified from: 'Endocrine Control of Nutrient Partitioning' by S Shadid and MD \\ Jensen; Book chapter for 'Obesity: Clinical Evaluation and Treatment', edited by Robert H. \\ Eckel, MD. Lippincott, Williams and Wilkins, Feb 2003.
}

\subsubsection{Introduction}

Understanding the regulation of energy balance and nutrient partitioning can potentially facilitate the treatment of obesity. The factors that lead to an imbalance between energy/fat intake and energy expenditure, and thus the development of obesity, remain incompletely understood. Some progress has been made in understanding the regulation of nutrient partitioning, e.g., the process by which the organism selects fuels for storage (including protein synthesis) or oxidation. Nutrient partitioning may be especially relevant to the development of obesity as it relates to the hypothesis of Dr. J.P. Flatt (104), who suggests that total food intake increases to meet carbohydrate needs. According to this theory, food intake is regulated, at least in part, to assure an adequate amount of carbohydrate. Consumption of a high fat diet would require the intake of excess fat in order to satisfy carbohydrate needs and therefore lead to obesity under this scenario.

The concept of a diet "relatively" deficient in carbohydrate becomes important in that dysregulation of substrate partitioning could potentially affect the body's sense of what constitutes adequate carbohydrate intake. For example, if fat were preferentially shunted towards storage, more carbohydrate would be required to meet oxidative needs, thereby preventing sufficient repletion of glycogen stores. This process is proposed to generate signals that stimulate appetite. Other examples where nutrient partitioning relate to obesity and body fat content include the stimulation of lean tissue synthesis at the expense of fat calories by androgens and growth hormone, and (presumably) the reverse of this process by deficiencies of these hormones.

Endocrine disturbances have long been associated with either the development of obesity and/or the result of obesity. Examples include Cushing's syndrome, thyroid disorders, or insulin resistance and polycystic ovarian syndrome. In some cases endocrine influences may alter fat content and body composition by altering nutrient management without markedly changing either energy intake or expenditure. In other instances, hormones influence body composition by changing energy expenditure (e.g. thyroxine).

Another point of interest is body fat distribution, a determinant of obesity-induced morbidity that is as important as the absolute amount of body fat. Upper body obesity, which usually connotes visceral obesity, is more strongly associated with insulin resistance, hypertension, coagulation abnormalities, dyslipidemia and cardiovascular death than obesity per se. Lower body obesity, in which fat preferentially accumulates in the gluteo-femoral region, is less strongly associated with these health hazards. Because endocrine influences may affect fat distribution these effects will be reviewed where information is available. 


\subsubsection{General Considerations}

When energy intake exceeds energy expenditure the excess calories must be stored. Excess energy intake in sedentary, hormonally stable adults almost inevitably results in the expansion of adipose tissue triglyceride stores. Circumstances that promote lean tissue accretion, however, can allow excess energy to be stored as muscle and/or visceral proteins. The most common circumstance resulting in net lean tissue accretion with excess food intake is increased physical activity, usually in the form of resistance exercise training. The initiation of endurance exercise training in a previously sedentary individual can have similar, albeit less pronounced effects on lean body mass. Finally, during the recovery from catabolic illness, repletion of lost body proteins is an important fate of excess energy.

Alterations in nutrient partitioning can also occur at a relatively stable weight. For example, the reduction in protein synthesis and increased fat accumulation with aging can be considered a form of nutrient partitioning. Whether the changes are driven solely by lesser physical activity or are aggravated by other (e.g. hormonal) factors is not clear. In summary, ingested nutrients can be directed

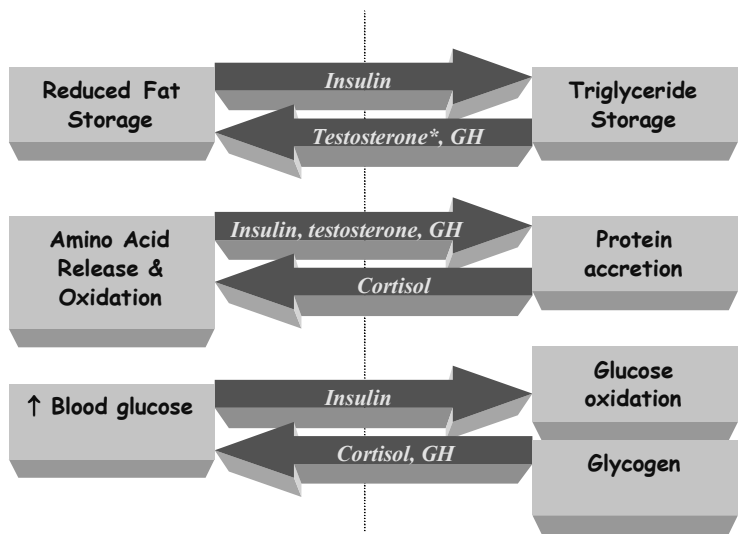

Figure 3.1 Endocrine influences on fuel balance

towards increasing body protein, increasing body fat or, to a much lesser degree, expanding carbohydrate stores. A variety of physiological and cellular events play key roles in determining nutrient partitioning. After examining the regulation of the major pathways of nutrient partitioning the specific hormonal regulation of each pathway will be reviewed. The net effects of the major nutrient partitioning hormones (insulin, growth hormone, testosterone and cortisol) are depicted in Figure 3.1.

\subsubsection{Processing Of Fuel: Fat, Carbohydrate and Protein Metabolism}

The main sources of dietary energy are carbohydrates $(4 \mathrm{kcal} / \mathrm{g})$, proteins $(4 \mathrm{kcal} / \mathrm{g})$, fat $(9$ $\mathrm{kcal} / \mathrm{g}$ ) and alcohol $(7 \mathrm{kcal} / \mathrm{g})$. Alcohol will not be discussed. Fat, carbohydrates and protein can serve functions in addition to fuel provision and the ultimate fate of these molecules depends on a variety of factors, among which hormonal influences are of importance.

\section{A. Fat metabolism}

Fat metabolism is also described in Chapter 2.1.Dietary triglycerides are transported in chylomicrons for storage or oxidation. Effective utilization of triglycerides requires the action of lipoprotein lipase (LPL), which is present on the capillary endothelium of many tissues. Adipose tissue and muscle are the major tissues responsible for triglyceride clearance by LPL mediated fatty acid uptake. Very low density lipoprotein (VLDL), secreted by the liver, is the other major form of circulating triglyceride. The fatty acids in these triglycerides derive largely from plasma free fatty acids (FFA) as described below. 
Fat stored in adipocytes is mobilized by the action of Hormone Sensitive Lipase (HSL), which hydrolyzes the triglyceride, resulting in the release of glycerol and FFA. Free fatty acids circulate bound to albumin, are present at relatively low concentrations $(300-600 \mu \mathrm{mol} / \mathrm{L}$ in the postabsorptive state) and turnover rapidly in the circulation (half-life of $\sim 4 \mathrm{~min}$ ). Uptake of FFA can be for use as an oxidative substrate (heart, muscle, etc.) or for reesterification (e.g. in liver) and subsequent transport in VLDL particles. At rest, FFA release and uptake rates generally exceed fatty acid oxidation rates. Hepatic re-esterification of FFA and secretion as VLDL triglyceride can account for a portion of the FFA that are released in excess of fatty acid oxidation. It is thought that substantial portions of VLDL triglyceride fatty acids are re-stored in adipose tissue.

Under conditions of grossly excessive carbohydrate intake glucose can be converted to fatty acids. This process is referred to as de novo lipogenesis, and is thought to take place primarily in the liver. It is energetically inefficient and accounts for a very small fraction of the fatty acids present in VLDL triglyceride under usual conditions.

The availability of FFA in the circulation drives fatty acid oxidation to a significant extent, as shown by the coordinate changes in fatty acid oxidation resulting from artificial elevation and lowering of plasma FFA concentrations. Some of the hormones that influence substrate partitioning may do so by in large part by altering FFA release from adipose tissue. For example, growth hormone stimulates lipolysis whereas insulin inhibits lipolysis, which in turn promotes and inhibits fatty acid oxidation. Fatty acid oxidation can also be regulated at other steps, however. It is possible for hormones to inhibit or stimulate fatty acid oxidation over and above their affects on FFA availability, thus sparing carbohydrate as an oxidative fuel. Altering the portion of fatty acids that enter re-esterification pathways or altering fatty acid transport into mitochondria could modify fatty acid (and thus glucose) oxidation independent of circulating FFA availability.

\section{Regulation of lipolysis}

Because of the importance of FFA availability in determining substrate oxidation, a brief overview of adipose tissue lipolysis will be provided. Under overnight postabsorptive conditions adipose tissue lipolysis is restrained by the prevailing insulinemia; pharmacological inhibition of insulin secretion using somatostatin results in an approximate doubling of FFA release rates. Physiological hyperinsulinemia is capable of suppression FFA release rates by 80 $90 \%$ from basal levels, and FFA concentrations by $>90 \%$. Insulin resistant, obese individuals display greater maximal release of FFA during insulin withdrawal and lesser suppression of FFA during hyperinsulinemia. Catecholamines are also potent regulators of adipose tissue lipolysis. Epinephrine infusions can increase FFA release by 3-4 fold. This is similar to the stimulation of lipolysis that occurs during exercise. Catecholamines act through stimulation $\beta$ adrenergic receptors via a classic Gs-protein/cyclic-AMP mechanism that activates HSL (105). Other hormonal regulators of lipolysis include growth hormone and cortisol, although these are generally considered to be much less potent that insulin and catecholamines.

\section{B. Carbohydrate Metabolism}

Most dietary carbohydrate is converted into glucose before storage or oxidation. The liver is the only organ that stores carbohydrate as glycogen for eventual export as glucose. The regulation of hepatic glucose export is under exquisite control by a variety of hormones, although insulin and glucagon are considered the most potent acute regulators of glucose availability.

There is a limited capacity for glucose storage as glycogen; such that increasing carbohydrate intake beyond usual needs quickly leads to increased carbohydrate oxidation, with an attendant reduction in fat oxidation. Increasing carbohydrate intake in the face of unchanging fat 
intake will result in increased fat storage as glucose replaces fat as an oxidative fuel. Massive increases in carbohydrate intake can stimulate de novo lipogenesis, although the capacity for this appears quite limited in humans (see above).

Reductions in carbohydrate intake are accompanied by some degree of glycogen depletion and shifts towards greater fat oxidation. If energy intake is less than energy expenditure fatty acids from pre-existing stores are oxidized resulting in relatively good preservation of protein stores. The shifts in carbohydrate and fatty acid oxidation that take place with changes in carbohydrate intake appear to be primarily regulated by the changes in insulin secretion that accompany the dietary changes.

\section{Protein Metabolism}

Proteins include structural proteins, enzymes, nucleoproteins, oxygen-transporting proteins, contractile proteins etc. The amino acids in these proteins are part of an interchangeable pool. The daily turnover of amino acids is substantially greater than the amount ingested as dietary protein; only a minor fraction of amino acids are oxidized or converted to glucose or fat.

Mechanical stimuli enhance skeletal muscle contractile protein synthesis, whereas hormonal and other factors determine the synthesis rates of many types of proteins. Given the tremendous variety of proteins in the body, many of which have specific regulatory pathways, this chapter will make only general reference to hormonal or metabolic regulation of protein metabolism.

Table 3.2.1 Hormonal effects on substrate partitioning and body fat distribution. ${ }^{1}$ insulin counteracts lipolytic effects of cortisol change? unknown

\begin{tabular}{|c|c|c|c|c|c|}
\hline Effect & Insulin & GH & Estrogen & Testosterone & Cortisol \\
\hline \multicolumn{6}{|l|}{ Energy Expenditure or fuel mix } \\
\hline REE/RQ & $\uparrow / \uparrow$ & $\leftrightarrow / \downarrow$ & $\leftrightarrow / \leftrightarrow$ & $\leftrightarrow / \downarrow$ & $\leftrightarrow / \leftrightarrow$ \\
\hline TEF/RQ & $\uparrow / \uparrow$ & $? / \downarrow$ & ? & $?$ & ? \\
\hline $\mathrm{PAEE} / \mathrm{RQ}$ & $\leftrightarrow / \uparrow$ & $?$ & $?$ & $?$ & $?$ \\
\hline \multicolumn{6}{|l|}{ Plasma substrate availability } \\
\hline Fatty acids & $\downarrow$ & $\uparrow$ & $\downarrow$ & $\leftrightarrow$ & $\uparrow / \leftrightarrow^{1}$ \\
\hline Glucose & $\downarrow$ & $\uparrow$ & $\leftrightarrow$ & $\leftrightarrow$ & $\uparrow$ \\
\hline Amino acids & $\downarrow$ & $\leftrightarrow$ & $\leftrightarrow$ & $\leftrightarrow$ & $\uparrow$ \\
\hline \multicolumn{6}{|l|}{ Direct intracellular effects } \\
\hline Fatty acid oxidation & $\downarrow$ & $\uparrow$ & $\leftrightarrow$ & $\uparrow$ & $\uparrow$ or $\leftrightarrow$ \\
\hline $\begin{array}{l}\text { Glucose (oxidation vs. glycogen } \\
\text { synthesis) }\end{array}$ & $\uparrow$ & $\downarrow$ & $\leftrightarrow$ & $\downarrow$ & $\downarrow$ or $\leftrightarrow$ \\
\hline $\begin{array}{l}\text { Amino acids } \\
\text { (protein synthesis) }\end{array}$ & $?$ & $\uparrow$ & $\leftrightarrow$ & $\uparrow$ & $\downarrow$ \\
\hline Fat storage & $\uparrow$ & $\downarrow$ & $\uparrow$ & $\downarrow$ & $\uparrow$ \\
\hline Regional fat storage & $?$ & $?$ & $\begin{array}{l}\text { Gluteo- } \\
\text { femoral }\end{array}$ & Visceral & $\uparrow$ (truncal) \\
\hline
\end{tabular}

REE: resting Energy Expenditure, RQ: respiratory quotient, TEF: thermic effect of food, PAEE: physical activity induced energy expenditure. $\uparrow$ increase $\downarrow$ decrease $\leftrightarrow$ no

\subsubsection{Energy Expenditure and Metabolism}

Nutrient partitioning could be accompanied or exaggerated by changes in energy expenditure. Human energy expenditure is usually broken down into three components: basal metabolic rate (BMR), the thermic effect of food, and physical activity. Basal metabolic rate is deter- 
mined primarily by lean body mass, and thus hormonal effects that increase lean tissue will increase BMR. The thermic effect of food is determined primarily by the amount of carbohydrate and protein ingested, but may be reduced in insulin resistant states.

Physical activity thermogenesis is a product of the mass of the body moved and the amount of movement. Hormonal effects on energy expenditure and substrate oxidation could theoretically influence either the amount of energy expended in one of these components or the mix of fuels oxidized. To the extent that hormones influence either energy expenditure or substrate oxidation this issues will be covered.

A summary of the effects of insulin, growth hormone, cortisol, testosterone and estrogen on energy expenditure, nutrient availability and oxidation, as well as fat/regional fat storage is presented in Table 3.2.1.

\subsubsection{Endocrine Control of Fuel Partitioning}

\subsubsection{Insulin}

\section{A. Physiology}

\section{General considerations on fuel partitioning}

Insulin is perhaps the prototypical hormone as regards nutrient partitioning. It enhances energy preservation by limiting the release of fuel substrate from tissues into the circulation and thereby enhancing their net storage (Figure 3.2). This is accompanied by increasing cellular uptake of glucose and amino acids. Under usual circumstances major increases in insulin release occur only after meals, when exogenous fuels become available. This results in insulin's anti-catabolic action (limiting lipolysis, glycogenolysis and proteolysis) taking place under circumstances where adverse consequences of hyperinsulinemia, such as hypoglycemia, are avoided. The other important feature of insulin action is the promotion of glucose as the preferred oxidative substrate, sparing amino acids and fatty acids for protein and triglyceride synthesis, respectively.

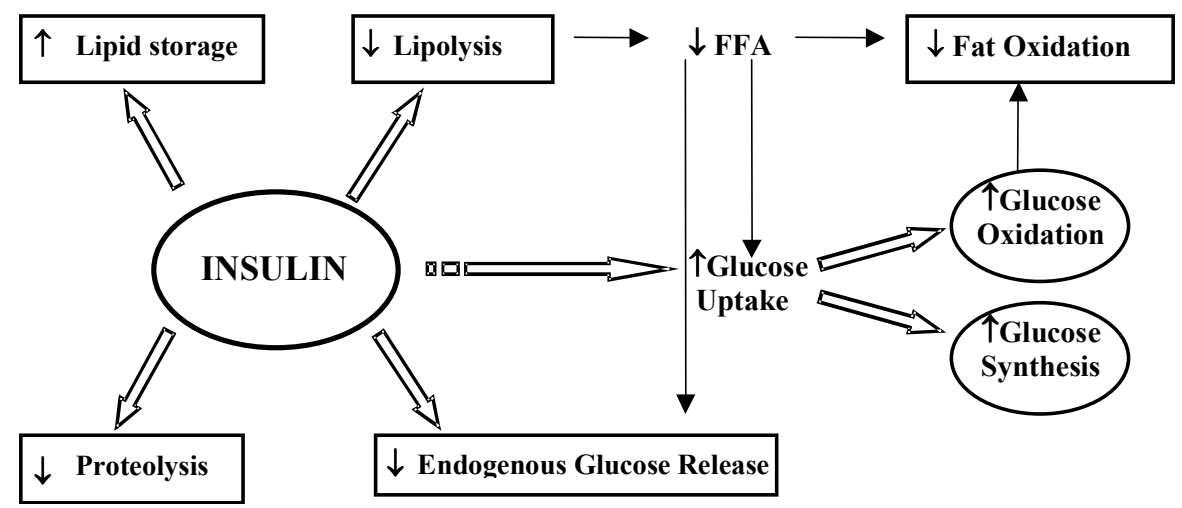

Figure 3.2. Insulin control of fuel partitioning 
Much focus has been placed on insulin's glucose metabolic action, although it is an equally important regulator of fat and protein metabolism. Insulin largely controls glucose uptake into most cells and stimulates both glucose oxidation and glycogen storage; skeletal muscle is the predominant site of postprandial glucose disposal. Insulin also suppresses endogenous glucose production by inhibiting hepatic glycogenolysis and gluconeogenesis. Thus, insulin largely orchestrates the disposal of meal carbohydrate by simultaneously inhibiting endogenous glucose production and stimulating the uptake and oxidation/storage of blood glucose.

As noted in the introduction, insulin also plays a major role in regulating lipolysis, and, likely via its ability to stimulate adipose tissue lipoprotein lipase synthesis, it facilitates adipose triglyceride uptake. It is unclear whether insulin has independent effects on FFA transport into cells. Whether insulin stimulates protein synthesis is somewhat controversial, however it is well established that insulin inhibits protein breakdown, and thus has the net effect of maintaining lean tissue mass.

In summary, insulin may be looked at largely as anti-catabolic rather than anabolic, although clearly the net effect is to preserve protein and fat stores. Insulin resistant states are of interest both with respect to their common presence in obesity, and because resistance to all aspects of insulin action may not appear in different insulin resistant states.

\section{Resting Energy Expenditure and the Thermic Effect of Food and Exercise}

Whether insulin modulates resting energy expenditure is a puzzling issue. Insulin deficiency, such as occurs with uncontrolled Type 1 or Type 2 diabetes, or with brief periods of fasting, is associated with slightly (5-10\%) higher REE even if lean tissue mass is appropriately accounted for. It has been suggested that the increase in REE is due to the higher rates of gluconeogenesis, which is an energetically inefficient process. Of interest, abrupt increases in insulin concentrations, such as occurs during euglycemic, hyperinsulinemic clamp studies also increase REE coincident with increased plasma catecholamine concentrations.

The increase in REE in response to hyperinsulinemia may be partly mediated via stimulation of the sympathetic nervous system; adrenergic blockade can inhibit this effect of insulin. The same adrenergically mediated mechanism may be responsible for the much of the insulin-mediated postprandial thermogenesis (106), although not all studies support this notion (107). Insulin resistance and obesity are associated with reduced postprandial thermogenesis (108). This is considered further evidence that insulin/insulin action modulates at least one, albeit minor, aspect of daily energy expenditure. This effect could potentially have long-term effects on energy balance and nutrient partitioning.

There is no data to address the issue of whether insulin modifies the thermic effect of exercise. Moderate intensity exercise normally results in a gradual decline in plasma insulin concentrations, which facilitates both glucose and FFA mobilization to provide fuel for exercising muscles. If insulin concentrations do not decline (or actually increase) carbohydrate oxidation increases above normal, requiring exogenous glucose to prevent hypoglycemia. The limited evidence available suggests that obesity, including more insulin resistant forms of obesity, have normal substrate oxidation responses to exercise despite somewhat different lipolytic responses. Thus, while insulin could potentially have drastic effects on nutrient partitioning during exercise, the actual magnitude and relative importance of variations in the insulin response to exercise is not well defined.

In summary, REE increases both in response to insulin deficiency and hyperinsulinemia, likely via completely different mechanisms. A significant portion of the thermic effect of food is mediated by the postprandial insulin response. It is unknown whether insulin alters the thermic response to exercise. Thus, abnormalities in insulin secretion or action can affect nu- 
trient partitioning both be altering energy expenditure and tissue selection of substrate oxidation and storage.

\section{Substrate availability and oxidation}

As mentioned above, insulin decreases the concentration of glucose, FFA and amino acids in the circulation. In the case of FFA this has direct consequences for substrate utilization in peripheral tissues, which are then forced to intensify their use of glucose as oxidative fuel. The suppression of adipose tissue lipolysis with the subsequent decrease in plasma FFA availability makes an important quantitative contribution to insulin's effects on peripheral glucose uptake and oxidation. This has a major impact on nutrient partitioning, in that ineffective suppression of lipolysis following meal ingestion can allow more fat and less glucose to be oxidized. This is proposed to be a mechanism by which the insulin resistance of obesity increases fat oxidation, thereby allowing the individual to eventually balance fat oxidation and fat intake.

The concept that failure to suppress fatty acid concentrations in the face of hyperinsulinemia will impair glucose disposal and oxidation has been confirmed. If the fall in FFA concentrations in response to hyperinsulinemia is prevented by infusing a lipid emulsion and heparin, glucose disposal and oxidation are not stimulated to the expected degree. In addition, normalizing the suppression of FFA in volunteers with Type 2 diabetes (whose lipolysis is normally quite resistant to insulin) by administering acipimox, a drug with antilipolytic properties, markedly enhances insulin action with respect to glucose metabolism (109).

Insulin also inhibits the release of amino acids from tissues into the circulation by inhibiting proteolysis. In the context of a mixed meal (i.e. one providing protein as well as carbohydrate) the plasma amino acid concentrations increase as a consequence of gut delivery of amino acid into the circulation. Increased amino acid availability plays a role in stimulating protein synthesis. If insulin concentrations increase from the ingestion of pure carbohydrate or during intravenous glucose and insulin infusions, plasma amino acid concentrations and protein synthesis rates fall. Thus, the nutrient partitioning effects of insulin with respect to protein metabolism are strongly influenced by the nutrient content of the challenge.

\section{Intracellular effects upon nutrient partitioning}

Insulin also promotes glucose oxidation and storage independent of its effects on substrate availability via direct action on intracellular events. Evidence for these effects has been provided by in vivo studies whereby FFA concentrations have been maintained (via an intravenous lipid and heparin infusion) during hyperinsulinemic, euglycemic conditions. In these experiments, glucose uptake, storage and oxidation increase above basal in response to insulin, although the rates do not equal those seen when FFA concentrations are allowed to fall normally (110). This response is clearly evident in skeletal muscle (110), but not in liver (111).

These direct intracellular effects of insulin are mediated by a complex signaling cascade that regulates glucose transporter availability at the cell membrane, as well as the activity and synthesis of numerous enzymes. The details of this process are beyond the scope of this chapter. Other direct intracellular effects of insulin include the stimulation of amino acid transport into cells, which should facilitate protein synthesis.

\section{Tissue variability in insulin action}

The effects of insulin on different tissues can vary considerably and in such a manner as to influence nutrient partitioning. For example, insulin regulation of glucose, protein and fatty acid metabolism can diverge considerably in fasting. Brief (3-4 days) of fasting in humans results in insulin resistance with respect to adipose tissue lipolysis (112) and glucose disposal 
(113), but maintenance of insulin's ability to suppress proteolysis (113) and enhanced suppression of endogenous glucose production (113). This physiological adaptation allows enhanced mobilization and oxidation of fatty acids while sparing peripheral glucose utilization (presumably allowing adequate glucose to meet central nervous system needs). It also preserves protein-sparing effects of insulin, thus minimizing lean tissue losses. In other insulin resistant states, such as upper body obesity, the insulin resistance appears to affect fatty acid, glucose and protein metabolism (114).

There are also inherent regional and tissue differences in insulin action that can potentially regulate regional nutrient partitioning. Skeletal muscle protein metabolism is affected differently by insulin than is splanchnic protein metabolism. Under overnight, postabsorptive conditions, when plasma insulin concentrations are at basal levels, skeletal muscle is a net exporter of amino acids (i.e. protein breakdown exceeds protein synthesis) whereas protein synthesis exceeds protein breakdown in the splanchnic bed (115). Increasing the plasma insulin concentration results in suppression of muscle protein breakdown without stimulating protein synthesis, but suppresses protein synthesis in the splanchnic bed without altering protein breakdown (115). The reduction in splanchnic protein synthesis in response to insulin may have been related to the fall in plasma amino acid concentrations resulting from isolated hyperinsulinemia. Thus, partitioning of amino acids between skeletal muscle and non-skeletal muscle sources is at least partially regulated by insulin.

Regional differences in insulin regulation of adipose tissue lipolysis are also present in humans. Leg adipose tissue lipolysis is readily suppressed by insulin (63), with upper body subcutaneous and visceral adipose tissue FFA release being less well inhibited (63). This is true in both lean and obese humans $(43,63)$. If regional adipose tissue fatty acid storage were equal in all adipose tissue beds one would expect gradual expansion of lower body fat stores. Studies of leg, abdominal subcutaneous and visceral adipose tissue indicate that regional variations in fatty acid uptake are in the same direction as regional variations as FFA release (52). Thus, whether regional differences in insulin action contribute to differences in body fat distribution is unknown.

\section{B. Insulin and nutrient partitioning in obesity}

Insulin resistance with respect to glucose and fatty acid metabolism is an exceeding common feature of obesity, being more severe and more prevalent in upper body/visceral obesity. As noted above, this insulin resistance with respect to adipose tissue (less stimulation of LPL synthesis, less suppression of lipolysis) would theoretically act to make more lipid fuel available, thus displacing glucose as an oxidative substrate. If there is a sensing mechanism whereby the internal stimuli for energy intake are modulated by the availability of glucose, the reduced carbohydrate oxidation resulting from insulin resistance could serve to limit excess energy intake. In this regard, insulin resistance has been proposed as a mechanism to limit weight gain (116).

The development of Type 2 diabetes in obese adults results in a host of nutrient partitioning changes. The relative insulin deficiency allows further excess mobilization of FFA from adipose tissue, which in theory would further depress glucose uptake and oxidation. The hyperglycemia itself, however, facilitates glucose uptake and oxidation independent of insulin, thereby limiting the extent to which fatty acid oxidation can increase. The loss of the restraining effect of insulin on skeletal muscle protein breakdown results in substantial losses of muscle in uncontrolled diabetes. In addition, glycosuria results in the non-oxidative loss of carbohydrate. This catabolic state with regards to adipose, muscle and carbohydrate can be reversed with insulin administration or insulin secretagogues at the expense of weight gain. 
This weight gain could well be muscle tissue and repletion of glycogen (with its attendant water), with limited adipose accumulation. Thus, the energy deposition that occurs when uncontrolled diabetes is treated is an excellent example of nutrient partitioning into lean tissue.

In summary, insulin is involved to a major extent in virtually every aspect of nutrient partitioning. The primary issue that will face clinicians with respect to this hormone and obesity is the weight gain that attends insulin or insulin secretagogue therapy for uncontrolled type 2 diabetes.

\subsubsection{Growth Hormone}

\section{A. Physiology}

\section{General considerations on fuel partitioning}

Growth hormone (GH, somatotropin) is secreted by the pituitary in response to hypothalamic regulation via Growth Hormone Releasing Hormone (GHRH) and Growth HormoneInhibitory Hormone (SRIH, somatostatin). Physiologic stimuli to secretion include starvation, hypoglycemia, exercise and stress; increased insulin like growth factor 1 (IGF-1), glucose and fatty acid concentrations inhibit GH secretion. GH is a major anabolic hormone. Its most important functions consist of fuel redistribution (Figure 3.3) and the induction of growth in all body cells with growth capacity. The latter is achieved by increasing cell size and number, largely via IGF-1, and in synergism with insulin, which enhances the transport of glucose and amino acids into cells.

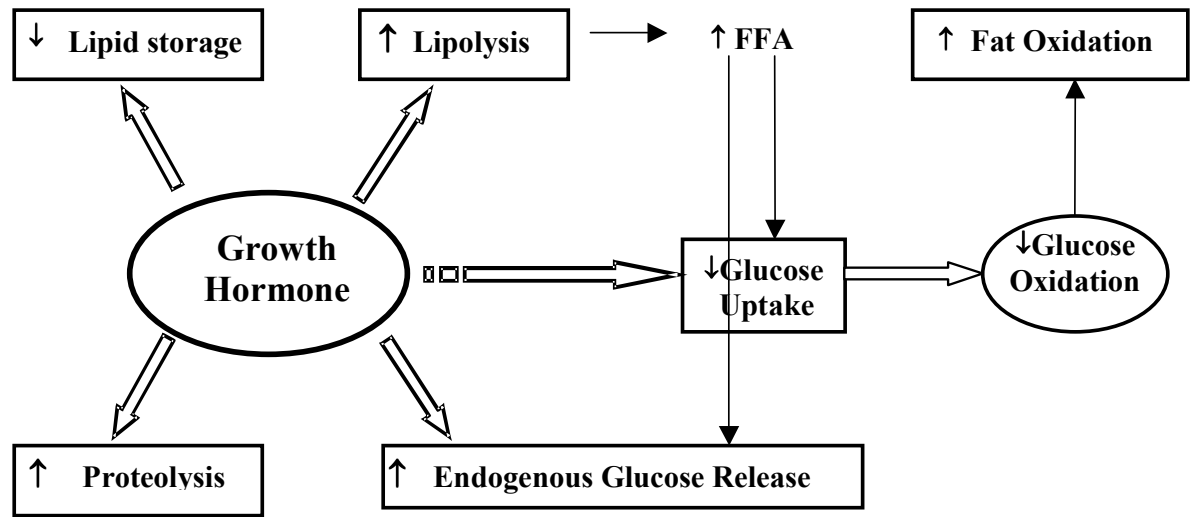

Figure 3.3. Growth hormone control of fuel partitioning

During puberty, sex hormones substantially increase GH production, which leads to the growth spurt and to the increase in lean body mass. For the latter, the synergistic interaction with testosterone is responsible for stimulation of protein synthesis and fat mobilization and oxidation.

The effects of GH on fuel partitioning are directed towards the use of fat as the preferred oxidative substrate, whilst minimizing the use of protein and carbohydrates for this function. GH stimulates fatty acid release from adipose tissue (117,118-120); GH deficient patients are, in concordance, reported to have an increased fat mass and a reduced muscle mass, which can be reversed back to normal after treatment $(119,121,122,123)$. Analogously, acromegalic patients 
have a reduced fat mass and increased lean body mass (LBM) (124), likely due to GH induced increases in protein synthesis. (see also 'substrate availability').

Growth hormone also increases plasma glucose concentrations, which cannot always be compensated for by increased insulin secretion. Thus, $\mathrm{GH}$ is one of the prototypical diabetogenic hormones.

\section{Insulin Like Growth Factors}

Many GH functions are mediated via Insulin Like Growth Factors or somatomedins, of which IGF-1 (somatomedin C) is the most important. These proteins are produced primarily in the liver and, to some extent, in some other GH responsive tissues where they exert autocrine and paracrine effects.

Although GH is the most important regulator of IGF release, the nutritional status may modulate the response: serum IGF concentrations gradually decrease with fasting and are consistently low in undernourished states. In obesity, IGF-1 is usually normal.

IGF-1 mediates the stimulatory effects of GH on growth, protein synthesis and whole body metabolism. Regulation of fat metabolism, however, seems to be IGF-1 independent (119): there are indications that adipocytes do not possess functional IGF-1 or IGF-2 receptors in contrast to their abundant GH receptors (125).

IGF exert a negative feedback on GH secretion on hypothalamic and pituitary levels.

\section{Resting Energy Expenditure and the Thermic Effect of Food and Exercise}

As expected, the GH induced increase in LBM increases REE $(124,126)$. This has been confirmed in acromegalic patients $(119,127)$ and in GH deficient adults, children and abdominally obese men, in whom GH treatment increases REE $(128,129,130)$. This effect seems to result from changes in LBM only: In children, REE increased even after correction for FFM, but only during the first 2 months of GH administration, after which it stabilized despite sustained changes in body composition (130). Analogously, in adults REE returned to baseline values after an initial increase in the first 2 weeks of GH treatment (129). This suggests that $\mathrm{GH}$ does not influence resting metabolic rate independent of changes in LBM.

The thermic response to glucose has been reported to be blunted in acromegaly (126). This may be due to the effects of insulin resistance on TEF (see the section on insulin). Interestingly, TEF increased after GH therapy in GHD children (130), whereas in GHD adults no changes were found (127). No information is available regarding the potential effects of GH on physical activity thermogenesis.

\section{Substrate availability and oxidation}

The metabolic effects of GH are biphasic. In the first $\sim 90$ minutes after secretion, a transient insulin-like effect is observed, characterized by accelerated glucose uptake and oxidation (118). The physiological relevance and the mechanism of this phenomenon are unknown; however, once this effect has dissipated, the lipolytic and diabetogenic effects of GH become apparent.

The main effect of GH is to increase FFA and glucose availability to peripheral tissues. Fatty acids are mobilized from adipose tissue via stimulation of HSL, of which the action, but not the production is promoted (120). This is suggested to be mediated via increased adipocyte responsiveness to catecholamines $(131,132)$; however, whether this is entirely responsible for the increased lipolysis is unclear.

In addition, GH inhibits adipose tissue-LPL (117). This is reported to take place at a posttranslational level $(117,133)$; an influence on LPL gene expression has not been consistently 
observed in vivo or in vitro $(117,134)$. Whether GH affects skeletal muscle LPL is controversial $(117,132,134)$.

In addition to increasing FA availability, GH directly enhances fat oxidation, at least in part by stimulating the conversion of fatty acids to long chain acyl-CoA within responsive cells (135). These actions reduce the need for protein and carbohydrates as energy sources. Thus, cellular uptake and oxidation of glucose diminishes (119); glucose that enters the cell is deposited as glycogen. In some tissues GH directly inhibits glucose uptake independent of FFA concentrations (136).

Growth hormone also stimulates hepatic glucose production (119). Both acute and prolonged excess GH therefore causes plasma glucose concentrations to rise, which cannot always be compensated for by increased insulin release $(119,137)$.

GH is protein anabolic: it enhances protein synthesis by increasing cellular amino acid uptake in synergism with insulin $(135,138)$ and by modulating DNA and RNA transcription. Synergistic effects with testosterone make this more pronounced in males than in females (135). Although GH influences protein metabolism via IGF-1 $(119,135)$, the presence of both hormones together has a greater effect than each one separately (Kupfer in (138)).

Whether GH additionally inhibits proteolysis is controversial. Although many studies show no effect of GH on proteolysis (139), 6 hour GH infusions in healthy volunteers showed $\mathrm{GH}$ to suppress net forearm alanine, phenylalanine and leucine release respectively $(140,141)$. It is unknown whether this merely reflects a decreased amino acid efflux, secondary to their increased extraction from the circulation (141), or a true suppression of proteolysis. The latter has been demonstrated to be induced by IGF-1 in animal and human studies (Elahi, Laager, Umpleby in (138)). GH decreases amino acid oxidation by approximately $20 \%$, as shown by GH administration to GH deficient adults $(141,142)$ and children (119).

\section{Regional fat storage}

When administered to patients with primary growth hormone deficiency, GH redistributes fat from abdominal to peripheral depots. In GH deficient children and adults, lean body mass is less and visceral fat mass is increased (118). GH treatment has shown to reverse this centripetal fat deposition and, in children, to decrease fat cell size in abdominal (as opposed to gluteofemoral) depots (Brummer in (122)) (Tanner in (118)). Cessation of treatment reverses these effects. (Collip in (118)).

Analogously, acromegalic patients are found to have a lower total fat mass and increased FFM as a result of both increased total cell mass and increased extracellular fluid (122). After treatment of acromegaly intra-abdominal fat and total body fat increases $(118,122)$. The mechanism of the effect of $\mathrm{GH}$ effect on fat distribution (changes in regional mobilization vs. changes in regional storage) has not been investigated using in vivo approaches; however, in vitro studies offer some possible explanations for the observed effects.

There exists regional variability in LPL and/or HSL responsiveness to GH. Neither has been extensively studied. To our knowledge, only one study, investigating the effects of GH administration on adipose tissue LPL of obese women, studied gluteal and abdominal subcutaneous adipose tissue separately (134). In both regions, GH significantly inhibited LPL activity, which seemed more pronounced in gluteal than in visceral fat. Although this suggests a regional difference in sensitivity to GH inhibition of LPL, the two areas were not statistically compared and thus definite conclusions cannot be drawn. 


\section{B. Growth Hormone And Visceral Obesity}

The role of GH in obesity is complex and somewhat controversial. Although primary growth hormone deficiency leads to centripetal adiposity, visceral obesity per se results in a secondary reduction in serum GH concentrations (86). However, we view the latter as an adaptation to the state of energy surplus.

The differences in the pathophysiology of the two conditions are exemplified by the different IGF-1 concentrations. IGF-1 is very low in primary GH deficiency, but may be normal, high or somewhat reduced in obesity. In the latter case, IGF-1 concentrations are usually only slightly lowered from normal. Moreover, a simultaneous change in the production of IGFbinding proteins in central obesity (decreased IGFBP 1 and 2 versus increased IGFBP 3 ) may result in high free, biologically active IGF-1 fractions, exceeding those in lean subjects (Frystyk, Nam, Argente in (86)).

Therefore, IGF-dependent functions of GH, such as the growth ability of children, remain unchanged in obesity (86).

One could reasonably argue that reduced GH may dampen the tendency for high FFA concentrations in central obesity, thereby preventing further worsening of insulin resistance. Indeed, treatment of centrally obese adults with GH, while resulting in body fat loss, is almost uniformly associated with further increases in FFA and worsening glucose intolerance/insulin resistance.

The reasons for this hyposomatotropinism in obesity and its mechanisms have yet to be clarified. Reductions in spontaneous GH secretion (as much as $6 \%$ for each unit increase in BMI) and in the half-life of circulating GH have been reported (Iranmanesh, Veldhuis in (86)). Moreover, the GH response to pharmacological (GHRH, L-dopa) and physiological stimuli, such as sleep, physical exercise, insulin-induced hypoglycemia, and corticosteriods is impaired in adiposity (86).

Several theories have postulated explanations for the decreased GH concentrations, focusing on IGF-1, FFA, peripheral hormones and the central nervous system. Increased plasma concentrations of FFA and IGF-1 are observed in obesity, and both are known to provide negative feedback control on GH secretion. Thus, each might contribute to the hyposomatotropinism. Indeed, the administration of acipimox, an FFA-lowering nicotinic acid analogue, has been proven to increase spontaneous and stimulated GH release in obese subjects, both in acute and chronic application (86). There is no comparable support for the IGF-1 theory and the quantitative importance of either of these feedback loops in mediating the alterations in GH dynamics in obesity is unknown.

Other hormonal theories to explain the low serum GH in obesity concentrate on leptin and insulin. Because plasma concentrations of leptin rise as a function of percent body fat, an inhibiting effect on GH release has been suggested. However, conflicting in vitro evidence, showing both stimulatory and inhibitory effects of leptin on GH release, has challenged this theory (86). Altered leptin and GH concentrations are more likely to be separate reflections of obesity rather than interrelated phenomena with a cause and effect relationship.

The elevated plasma insulin concentrations, present in visceral obesity have also been proposed to inhibit the release (hypothalamus and pituitary gland) and the peripheral effects of GH. Nevertheless, a central role for insulin in the hyposomatotropinism in obesity has been weakened by the observation of normal GH levels in other hyperinsulinemic disorders such as hyperprolactinemia (143). 


\section{Growth Hormone Administration In Visceral Obesity}

The decrease in GH concentrations in obesity, and specifically visceral obesity, has led to experimental administration of this hormone, in an attempt to promote weight loss while preserving proteins and improving fat distribution (144-153). However, we found no evidence that these goals are truly reached. This is described in detail in Chapter 4.3.

\subsubsection{Testosterone}

\section{A. Physiology}

\section{General considerations on fuel partitioning}

Testosterone is a major anabolic hormone in humans (Figure 3.4). During male puberty dramatic increases in skeletal muscle mass and reductions in body fat occur, mediated by testosterone, perhaps acting in concert with growth hormone. Testosterone does not exert its effect directly, but is converted into dihydrotestosterone (DHT) or estrogens. Both function via nuclear receptors to modify gene transcription.

In addition to being responsible for the development of the primary and secondary sexual characteristics of the male, testosterone markedly influences nutrient partitioning.

Its most evident influence is on protein metabolism: it increases the rate of protein formation in target cells, most noticeably myocytes, thus promoting muscle development. Moreover, testosterone affects body fat distribution, decreases adipose tissue fatty acid uptake and may affect lipolysis, perhaps related to synergistic effects with GH. Testosterone increases fatty

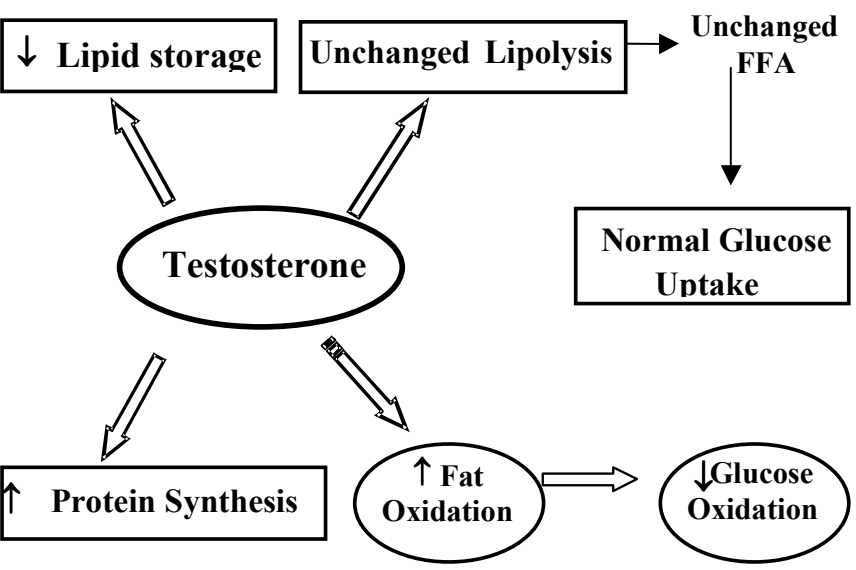

Figure 3.4. Testosterone control of fuel partitioning acid oxidation, and there is some evidence to suggest that this is a consequence of a direct enhancement of the efficiency of the oxidation of circulating fatty acids. Abrupt loss of testosterone in men rapidly results in fat gain and loss of lean tissue and muscle strength (154), emphasizing the importance of this hormone in directing substrate partitioning.

\section{Resting Energy Expenditure and the Thermic Effect of Food and Exercise}

The redistribution of the body fuel stores towards protein anabolism and away from fat storage results in a greater percent of the body as lean tissue. This accounts for the typical lower percentage body fat in men $(\sim 15 \%)$ compared to women $(\sim 30 \%)$ and their greater basal metabolic rate (and thus energy expenditure) relative to body weight. Testosterone has not been found to increase basal metabolic rate (BMR) beyond that explained by increased lean body mass (LBM) in healthy men. However, in a small group of men with muscular dystrophy, this 
did seem to be the case (155). Whether testosterone affects the other major components of daily energy expenditure (TEF or PAEE) has not been well studied.

\section{Substrate availability and oxidation}

The effects of testosterone on whole body protein and fat metabolism have primarily been studied using testosterone supplementation in hypogonadal men and adolescents with delayed puberty, as well as by the artificial induction of testosterone deficiency in lean, healthy young men $(154,156,157)$. The latter was achieved via the administration of $\mathrm{GnRH}$ analogues. In these experiments, in which growth hormone and IGF-1 remained unchanged, a rapid decline in whole body protein synthesis and proteolysis was observed, resulting in a decrease in fatfree mass and muscle strength (154). Testosterone supplementation studies have confirmed these conclusions, except for the effects on proteolysis, which was found to decline under the influence of testosterone.

In all studies, amino acid oxidation rates and plasma amino acid concentrations did not change, suggesting that testosterone amplifies muscle mass by enhancing intracellular partitioning of amino acids towards protein synthesis (especially in muscle) rather than by increasing amino acid uptake. The latter has been confirmed in amino acid tracer studies of testosterone administration in healthy young men (158). It should be noted, however, that these studies are conducted in fasted states and that, under physiological conditions, cellular amino acid availability and uptake are expected to increase due to postprandial fluctuations in other hormones such as insulin and $\mathrm{GH}$.

Acute testosterone deficiency diminished rates of lipid oxidation and resting energy expenditure, but increased body fat mass. This suggests that testosterone-induced increases in fatty acid oxidation are not entirely due to the greater muscle mass.

It is not clear to what extent these changes in fat oxidation result from direct effects on intracellular fatty acid trafficking or from changes in FFA availability. Although testosterone increases adipocyte lipolytic responsiveness to catecholamines (norepinephrine, isoproterenol and forskolin) in in vivo and in vitro studies $(159,160)$, basal lipolytic rates are not influenced. In addition, in vivo markers of basal lipolysis remain unchanged in some testosterone supplementation studies (156).

Fat deposition is greatly influenced by testosterone, via its inhibition of LPL (160); this shall be further discussed in 'regional fat storage'.

No evident changes have been observed in serum glucose levels or glucose availability after the induction of hypogonadism in healthy men. Insulin concentrations tended to increase with acute testosterone deficiency (154). Of interest, testosterone treatment in adolescents with delayed puberty causes insulin stimulated oxidative and non-oxidative glucose disposal rates to decline (156). These effects could well be temporary, as is the insulin resistance of puberty, and may be related to increased competition from greater fatty acid oxidation. In obese men, who are known to have lower plasma testosterone levels, testosterone administration increased insulin stimulated glucose disposal rates (161). Whether this is a direct or an indirect effect, perhaps relating to changes in fat distribution or adipose tissue function is not clear.

In summary, testosterone increases the oxidation of fatty acids, but not of amino acids. The typical protein anabolic effects result from altered intracellular trafficking rather than increased uptake of amino acids. The reported small effects on glucose metabolism may be indirect results of fat re-distribution. 


\section{Regional fat storage}

Testosterone has distinct effects on body fat distribution, although the androgen/fat distribution association varies with sex and stage of development. The increase in testosterone during male puberty is associated with an increase in the portion of intra-abdominally stored fat with a concomitant reduction in the relative amount of fat in the lower body. Analogously, adult women with excess androgens have a similar increase in intra-abdominal fat, and women who develop visceral obesity are found to have increased serum concentrations of free testosterone.

In contrast, adult hypogonadal men also have increased visceral fat that is reduced following testosterone replacement (162), and viscerally obese men have lower serum free testosterone concentrations $(91,163)$. Thus, one cannot make blanket statements about the effects of androgens on regional fat storage.

Testosterone may modify body fat distribution by altering regional fatty acid uptake or regional lipolysis, and there are indications that it influences both. Administration of testosterone to hypogonadal men and to viscerally obese men with low serum testosterone concentrations shifts fat deposition from intra-abdominal to subcutaneous sites (161). This is associated with concordant changes in regional meal fatty acid uptake as measured in radioactive tracer studies (164).

In addition, 6-weeks and 9 months respectively of testosterone administration decrease LPL activity, a putative mediator of regional fat uptake, in subcutaneous abdominal, but not in femoral fat $(92,160,165)$.

There is also some evidence for regional variability in lipolysis as a result of testosterone "replacement". Testosterone administration decreased the half-life of fatty acids in abdominal, as opposed to femoral, subcutaneous fat in men with lower serum testosterone concentrations (164). Although these radioactive tracer studies suggested that increased lipolysis occurred with testosterone treatment, reductions in uptake combined with reductions in regional fat mass could give the same appearance.

Since many studies on the effects of testosterone on regional fat distribution have been conducted in obesity, in which testosterone dynamics are notoriously altered, extrapolation of the above-mentioned results to healthy, lean subjects should be done with caution. However, the fact that, in healthy men fat typically accumulates in the upper body, seems to be a typical effect of testosterone and indicates that this extrapolation is justified. Moreover, similar effects of testosterone on fat distribution have been found in physiological animal studies. Among others, it has been shown that intra-abdominal fat depots have a higher density of androgen receptors than subcutaneous depots in rats $(105,166)$.

\section{B. Testosterone and visceral obesity}

Testosterone deficiency leads to visceral fat accumulation; however, analogously to GH, visceral obesity as a primary entity, is also associated with lower free testosterone levels in men (91). As noted above, however, in women visceral obesity is associated with increased free testosterone (1073 . A satisfactory explanation for this paradox has yet to be found; in neither case it is clear whether this is based on a cause-and-effect relationship, and if so, whether the central fat accumulation leads to changed testosterone levels or vice versa. In both sexes, weight loss has been consistently found to reverse the abnormalities in testosterone levels $(167,168,169,170)$.

Several theories have postulated hormones such as leptin and GH to mediate the observed changes. However, neither is compatible with the changes in testosterone levels in women $(171,172)$. 
In conclusion, upper body obesity is associated with reversible high testosterone levels in women but low levels in men. Though the latter relationship has been intensively investigated, insight in the mechanism remains poor, theories varying from direct cause-and-effect relationships to indirect effects involving leptin and $\mathrm{GH}$.

\section{Testosterone administration in visceral obesity}

Given the known effects of testosterone on nutrient partitioning and the finding that visceral obesity in men is associated with reduced testosterone, it is logical to consider testosterone as therapy for obese men. In Chapter 4.2, we review study reports on this approach.

\subsubsection{Estrogens}

\section{A. Physiology}

\section{General considerations on fuel partitioning}

Estrogens are produced primarily in the ovaries, although to some extent the adrenals and adipose tissue can produce estrogen via conversion of androgens. In humans, the most important estrogen is $\beta$-estradiol.

In addition to their classic role in the development of female sex characteristics, estrogens exert mild anabolic effects on fuel partitioning, the most noticeable effects being on body fat and body fat distribution. During female puberty a redistribution of fat to the gluteo-femoral region occurs, which is thought to be estrogen mediated. Despite maintaining substantially more body fat (average of 30\% in non-obese women compared with $15 \%$ in non-obese men), women have less visceral fat and greater amounts of lower body fat than men do. Despite having more body fat, women typically have lower cardiac risk factors (serum lipids, etc.). This gender difference persists throughout the adult reproductive life but begins to reverse after menopause.

Whether estrogens directly influence the moderate anabolic effects and other aspects of fuel partitioning associated with their presence is controversial. Despite the common presence of a physiological deficiency state (menopause) that should facilitate research into these issues, the interpretation of study results is complicated by several factors.

First, the effects of estrogens on fuel partitioning are subtle compared to, e.g. those of testosterone, and therefore more difficult to determine. Small changes might be noticeable within individuals but might be more difficult to detect in cross sectional studies. Thus, longitudinal study approaches are most suitable for investigations into these issues; however, crosssectional studies are easier to conduct and thus more abundant. The results are sometimes conflicting.

Other methodological problems have added to the difficulty in identifying a nutrient partitioning effect of estrogens, including the enormous variability in research techniques to measure similar variables. For example, intra-abdominal fat accumulation has been assessed by waist circumference, waist-hip ratio (WHR), computed tomography (CT), magnetic resonance imaging (MRI) and dual energy x-ray absorptiometry (DEXA), all having different accuracy and reproducibility levels. In addition, in many studies confounding factors such as age have not been accounted for in the analyses.

Finally, estrogens interact with other hormonal systems. In addition to the obvious (cyclic and therapeutic) interaction with progesterone, estrogens decrease free testosterone concentra- 
tions, but increase GH production. These contrasting influences on two strongly anabolic hormones might blur direct measurable effects of estrogens in in vivo studies.

For example, estrogen stimulated GH production is known to be vital for growth, especially the growth spurt during puberty (154). In adult life, this interaction may influence fuel partitioning. However, estrogen administration has been found to increase GH production if given orally, but not transdermally $(173,174,174)$. Although the reason for this difference is not completely understood, it might influence the apparent effects of estrogen on fuel partitioning. It is unclear to what extent estrogen effects are exerted by interactions (either in synergism or indirectly) with GH and testosterone. Although estrogens would appear to have independent nutrient partitioning effects, one could argue that the gender differences in fuel partitioning result primarily from the difference in testosterone concentrations. This seems unlikely given that hypogonadal men have a body fat content and distribution that differs distinctly from women. In addition, although aspects of body composition change in post-menopausal women, they do not have the same nutrient partitioning as males. Therefore, though certain aspects of estrogen function occur in interaction with $\mathrm{GH}$, testosterone, progesterone and many other factors, a modest but direct effect on nutrient partitioning seems likely.

\section{Resting Energy Expenditure and the Thermic Effect of Food and Exercise}

Resting energy expenditure has been reported to increase, decrease or to remain unchanged under the influence of estrogen $(175,176,177,178)$. However, these conclusions are also mostly based on intra-menstrual comparisons or on indirect correlations; one of the few longitudinal studies reported a decrease in BMR of $100 \mathrm{kcal}$ per day after menopause, but was confounded by lower FFM and physical activity levels in the postmenopausal group (175). Estrogens seem to increase absolute LBM $(175,179)$ and thus REE (see 'body composition').

The thermic effect of food has been found to increase during the follicular phase of the menstrual cycle, likely due to the effects of progesterone (180). In obese women, glucose-induced thermogenesis was unrelated to estrogen levels when corrected for FFM (177). Although there are studies of menstrual cycle effects and oral contraceptive effects on substrate oxidation during exercise, there appear to be no studies of the thermic effect of exercise or nutrient partitioning during exercise in estrogen replete vs. estrogen deficient women. Thus, it is not know what effects estrogen have on nutrient partitioning during exercise.

\section{Substrate availability}

The influence of estrogens on protein metabolism is controversial, but there appears to be litthe if any effect of estrogen on circulating amino acid availability. Estrogen administration to hypogonadal girls (181) and inhibition of estrogen synthesis in young men (182) via the aromatase inhibitor anastrozole did not affect whole body protein pools, amino acid turnover rates or amino acid oxidation. In that study (182), estrogen inhibition increased testosterone concentrations and decreased IGF by $18 \%$, despite unchanged GH and IGFBP-3 concentrations.

There is no question that estrogen modulates the synthesis of selected hepatic, endometrial and other proteins in humans (181). The effects of estrogen on these selected protein pools occur without changing whole body protein kinetics, however, perhaps because the tissues strongly affected account for a relatively minor fraction of total protein synthetic activity.

The effect of estrogens on adipose tissue fuel export has been examined, however whether estrogen modulates adipose triglyceride uptake in humans does not appear to have been studied. Estrogens have been reported to increase, decrease or not to change lipolysis, both in animal and human studies $(105,183)$. Intervention studies have shown that transdermal estro- 
gen decreases adipose tissue FFA release in postmenopausal women (184) and lowers plasma FFA concentrations in ovariectomized women (185).

Neither glucose production nor insulin-mediated glucose disposal have been shown to be influenced by estrogens in humans. Contrary to the decrease in glucose disposal found in ovariectomized rats and mice (186), insulin sensitivity in post-menopausal women is reported to be unchanged or increased (Walton in (186)). Longitudinal studies of women going through menopause found unchanged glucose tolerance (Matthews in (187)) and increased fasting plasma insulin concentrations, but unchanged fasting glucose (175). Changes in fasting insulin in the peri-menopausal time period are confounded by changes in body fat and body fat distribution that commonly occur at this time of life (175).

The ability to draw unshakable conclusions from the available studies is limited by the variability in insulin sensitivity measurements (fasting plasma glucose to insulin clamp techniques), by the methodological difficulties mentioned above and, possibly, by the fact that studies are conducted too early in menopause to observe differences. Nevertheless, estrogen replacement studies lead to similar conclusions; it is therefore safe to state that the impact of estrogen on glucose metabolism in humans is too subtle to be clinically significant, if present at all.

\section{Substrate oxidation}

Conflicting data are available from human studies as to whether estrogen alters the partitioning of fat and glucose towards oxidation. One group reported that combined estrogen/progesterone supplementation of postmenopausal women did not alter basal substrate oxidation (173), but that after a mixed meal, carbohydrate oxidation increased at the expense of lipid oxidation. This change occurred only at 30-60 min after meal ingestion, and only in the group of women given oral estrogens. Transdermal estrogen administration resulted in an opposite, but non-significant effect. Another study failed to find effects of transdermal estrogen treatment on substrate oxidation under basal, insulin clamp or adrenergic stimulation conditions (184). Inhibition of estrogen synthesis in men with an aromatase inhibitor tended ( $\mathrm{P}=$ 0.09 ) to reduce basal lipid oxidation (182).

In conclusion, estrogens affect FFA availability and perhaps substrate oxidation. Estrogen deficient women have greater adipose tissue lipolysis (but not necessarily greater fat oxidation) than estrogen replete women. Estrogens selectively increase protein synthesis (in some tissues) without substantially changing whole body protein synthesis. Contrary to animal studies, estrogens appear to have little, if any influence on insulin-mediated glucose disposal in humans.

\section{Body Composition}

Although it seems clear that estrogens increase body fat, this is not so for visceral fat. This could be relevant to the reduced cardiovascular health risks in women. This question has mainly been investigated in the menopausal transition and is particularly subject to the methodological difficulties described above.

The cross sectional studies have reported that visceral fat either increases after menopause or remains unchanged; no reports of decreased visceral fat were found (186). Studies using CT and MRI, techniques with better accuracy and reproducibility tend to report a postmenopausal increase in intra-abdominal fat.

Only two longitudinal studies confirmed post-menopausal increases in waist circumference and WHR, with unchanged BMI. The first retrospectively re-analyzed a longitudinal study of 1462 women (188); the second monitored a cohort of 35 women for 6 years, during which 
half of the women experienced menopause. The latter lost more FFM and RMR than the others but kept comparable fasted glucose levels (175). These post-menopausal women were also found to have a decreased level of physical activity, which could have contributed to the observed changes.

This decrease in FFM following menopause has been confirmed by some, but not all crosssectional investigations (186). Intervention studies, however, have found that estrogen/ progesterone combination therapy prevents the post-menopausal increase in body fat (189) and increases muscle mass and creatinine excretion in postmenopausal women (179). The unchanged body weight implies a shift in body composition away from fat toward muscle. In an exception to this pattern, one study reported that oral estrogen replacement in postmenopausal women resulted in a significant reduction in lean tissue with a gain in fat tissue, a pattern not seen with transdermal estrogen replacement (173).

In conclusion, despite some conflicting results, longitudinal and interventional investigations lead to believe that estrogen preserve FFM in postmenopausal women, although the mechanism is not clear.

\section{Regional fat storage}

Many authorities believe the gender-related distribution of body fat can be at least partly attributed to the regional variability in the sensitivity of LPL to estrogens. Their inhibitory effect on adipose tissue-LPL has first been demonstrated in rat adipose tissue (Gray in (165)) and was confirmed in vivo in obese women (190). In the latter, a BMI-independent negative correlation was found between plasma estradiol concentrations and LPL activity, both in gluteal adipose tissue and in post-heparin plasma. In addition, in lean and obese men, femoral adipose tissue-LPL has been found to be more susceptible to suppression by estrogens and testosterone than its abdominal counterpart (165).

These results can account for part of the intra-abdominal fat accumulation in men, in whom testosterone effects on adipose tissue are mediated via aromatization into estrogens. In women, femoral and gluteal adipose cells contain more LPL per cell and per gram fat than abdominal fat cells (165), although the same is true in lean men (52). In addition, the influence of progesterone on adipose tissue progesterone receptors increases femoral fat accumulation (165). Whether estrogen influences regional variability in other factors that might modulate regional fat distribution, such as adipose tissue lipolysis, to our knowledge, is unknown.

\section{B. Estrogen Replacement Therapy And Nutrient Partitioning}

The same difficulties, encountered in the study of body fat distribution are found in the study of estrogen replacement and its effects on fuel partitioning, with, analogously, contradicting results as a consequence (187). A large meta-analysis concluded that the many available studies provide insufficient evidence for dependable conclusions on the effects of estrogen replacement therapy on WHR, fat mass and skin fold thickness (191). Interestingly, this study concluded that neither menopause nor estrogen replacement therapy affected BMI, regardless of the therapeutic regiment chosen.

The study of the effects of HRT on body composition and fat distribution in postmenopausal women derives its relevance from the relationship of body fat with cardiovascular risk factors. Although HRT generally has favorable effects on serum lipid concentrations, the effects on insulin resistance and glucose disposal are more difficult to determine: the methodological problems described earlier are augmented by small sample sizes, short duration of treatment and variable, often suboptimal methods for measuring insulin sensitivity.

The gold standard for the latter, the hyperinsulinemic euglycemic clamp technique, was used by one (cross-over) study only, reporting unchanged glucose disposal in postmenopausal 
women, both after transdermal and oral estrogen mono-therapy (174). Transdermal estrogen did, however, lead to greater FFA suppression during the clamp, suggesting an improvement in insulin action on adipose tissue anti- lipolysis or increased clearance of FFA. Treatment of women with Type 2 diabetes with estrogen for 6-weeks caused a slight but significant decrease in HbA1C without changing insulin mediated glucose uptake or plasma FFA concentrations (192).

The concomitant use of progestins clouds the evaluation of estrogen replacement on insulin sensitivity. In animal studies, progestins appear to decrease insulin sensitivity, confirming some, but not all, human studies in which they show a tendency to attenuate beneficial effects of estrogens on insulin sensitivity (Lindheim in (187)). The wide variety and dosages of progestins used make it difficult to draw firm conclusions regarding their effects on substrate partitioning. There is no strong evidence that HRT (combined or estrogen alone) alters substrate partitioning in a manner that alters total body fat mass, although HRT maintains a female fat distribution. Estrogen administration solely for this purpose does not seem indicated, and the decision to provide HRT should be based on traditional clinical considerations (osteoporosis, hot flashes, coagulation risks, etc.).

In conclusion, the beneficial effects of post-menopausal estrogen replacement therapy on serum lipids are well established. There is no proof that this is (causally) related to a decrease in intra-abdominal fat deposition, and total body fat is not affected. Some evidence exists for modest anabolic effects of estrogen on lean tissue. Despite reports on different influences on fuel partitioning and GH levels, there does not seem to be sufficiently convincing evidence in this regard that it is possible to recommend a specific route of estrogen administration.

\subsubsection{Glucocorticoids}

\section{A. Physiology}

\section{General considerations on fuel partitioning}

The major nutrient partitioning effects of excess glucocorticoids (95\% of endogenous glucocorticoid activity is cortisol) are of a catabolic nature (Figure 3.5). Their impact on fuel partitioning is particularly important in physical stress situations and exogenous (iatrogenic) or pathologic endogenous hypercortisolemia.

Cortisol increases the amount of fuel molecules in the circulation by inhibiting synthesis of glycogen, fat and skeletal muscle protein while increasing breakdown of the latter two. In addition, glucocorticoids stimulate appetite (likely via the suppression of Cortico-trophin Releasing Hormone; see below), providing the body with additional fuel.

The catabolic qualities of cortisol are far from random. Fat, protein and carbohydrate supplies are mobilized, re-stored and redirected according to their biological priority in stresssituations. For example, protein breakdown is disproportionately increased in many extrahepatic tissues. The liver uses the amino acids thus derived for enhanced protein synthesis, thus guaranteeing the production of acute phase proteins. Immunoglobulin synthesis in lymphocytes also increases.

With prolonged hypercortisolemia, protein catabolism progressively outpaces protein synthesis (193) (Maes in (194)). For example, in Cushing's syndrome protein wasting becomes especially evident in the skeletal muscle and skin. Furthermore, the decreased fat deposition in the extremities and increased fat accumulation in face and trunk lead to the classic fat redistribution of Cushing's syndrome despite greater fat mobilization. 


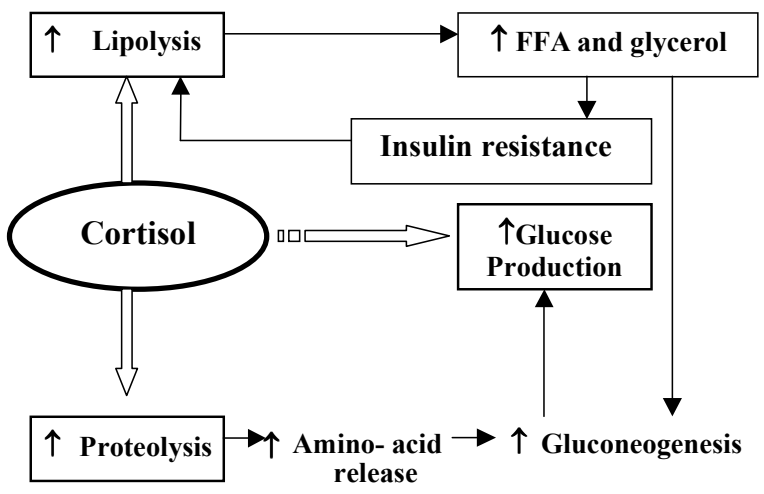

Figure 3.5. Cortisol control of fuel partitioning

Although the exact mechanism is not completely understood, there is evidence that the synthesis of enzymes responsible for adipocyte fatty acid uptake is differentially regulated by cortisol, possibly mediated by regional differences in expression and/or sensitivity of cortisol receptors.

Finally, prolonged and/or extreme corticosteroid excess can induce diabetes. The relative insulin deficiency of the diabetic state will further aggravate the protein catabolic effects of hypercortisolemia (195). Thus, the combination of corticosteroid excess and uncontrolled diabetes is particularly disadvantageous with respect to nutrient partitioning.

\section{Cortisol and the hypothalamic-pituitary-adrenal axis}

The interaction between the hypothalamus (corticotrophin releasing hormone, CRH), the pituitary (adrenocorticotropic hormone, ACTH) and the adrenals (glucocorticoids) provides the basic endocrine regulatory system, however, numerous modulating factors may be superimposed on this system and thus alter cortisol secretion. These internal and external conditions include illness and injury, hypoglycemia, psychological depression and environmental factors such as excess alcohol or nicotine and temperature (88). Hypothalamic nuclei integrate the various impulses (196).

In addition, the regulation of the hypothalamic-pituitary-adrenal (HPA) axis is intertwined with food intake and energy balance. Most types of hypercortisolemia increase appetite (197), which is thought to be mediated by feedback inhibition of corticosteriods on Corticotrophin Releasing Hormone (CRH) release.

$\mathrm{CRH}$ is a potent anorectic peptide, which, in rodent models, reduces the body weight threshold for food hoarding behavior (Cabanac in (198)). It is therefore thought to mediate the anorexia of primary adrenal insufficiency. In addition, obesity has been proposed to be a CRH deficient state (see below).

\section{Acute versus chronic effects}

The effects of chronic exposure to high physiological amounts of any hormone might differ from acute elevations. It is unclear to what extent these differences reflect a cumulative, but essentially unchanged effect of prolonged exposure, versus adaptations in enzymatic action, exhaustion, receptor modulation or even structural changes.

In the case of hypercortisolism these differences are particularly evident and readily visible. For example, the centripetal adiposity, buffalo hump and changes in hair pattern, are characteristic of chronic, but not acute hypercortisolemia. Furthermore, although changes in protein metabolism are evident with acute hypercortisolemia, over time structural changes can be observed in muscle. In addition to atrophy the muscle in Cushing's syndrome contains more type $\mathrm{II}_{\mathrm{b}}(32$ vs. $12 \%$ ) than type I fibers (30 vs. 55\%) compared with control subjects (199). 
Type $\mathrm{II}_{\mathrm{b}}$ fibers have a lower glycogen synthase activity and fewer mitochondria and are therefore thought to be more insulin resistant. Interestingly, upper body obese women display similar characteristics as women with Cushing's syndrome.

\section{Resting Energy Expenditure and the Thermic Effect of Food and Exercise}

REE has been reported to increase $(9-15 \%)$ by hydrocortisone infusions at physiological and supra-physiological infusion levels (80 and $200 \mu \mathrm{g} / \mathrm{kg} / \mathrm{hr}$ respectively) (193) (Bessey in (193)). Another report indicated that oral prednisone for one week did not increase REE (Horber in (193)); however, many factors complicate comparison of this study to the former, such as the duration and route of drug administration, the use of synthetic versus natural steroids and time points of measurement. To our knowledge, there is no information on the effects of cortisol on the thermic effects of food or exercise.

\section{Substrate availability and oxidation}

Hypercortisolemia increases plasma concentrations of glucose, amino acids and fatty acids. In stress situations this facilitates the delivery of fuel and substrate supplies to tissues, dependent upon these compounds to mount a host defense.

This is particularly evident in protein and fat metabolism, in which whole body catabolic effects of hypercortisolemia dominate the isolated, selective anabolic effects (193). In acute hypercortisolemia, increased protein breakdown has been well demonstrated $(193,200,201)$. Although whole body proteolysis increases $(193,201)$ and whole body protein synthesis decreases (138), synthesis of some proteins, such as some hepatic proteins and some lymphocyte proteins is selectively stimulated (Sato in (194)) (Grayson in (194)). Overall amino acid oxidation increases $(193,202)$.

With chronic hypercortisolemia whole body protein synthesis has been shown to decrease, whereas the effects on proteolysis are controversial $(138,195)$.

Analogously, acute hypercortisolemia has been demonstrated to have a net whole body lipolytic effect, increased FFA concentrations and a trend towards increased fat oxidation, despite simultaneous (and perhaps localized) stimulation of LPL (193).

Cortisol has been demonstrated to facilitate catecholamine-induced lipolysis in vivo, despite conflicting in vitro evidence $(203,204)$ (Rebuffé-Scrive and Fain in (199)). However, cortisol induced FFA release is counteracted by concomitant insulin overproduction (44). In chronic hypercortisolemia there is a net increase in fat mass and body weight (205).

Paradoxically, in some in vitro studies of human adipose tissue found increased LPL activity and fat uptake under the influence of cortisol (206) (Bjorntorp in (206)). These investigators suggested that small amounts of insulin are needed for this process (206), which may be important given the insulin resistance/hyperinsulinemia induced by excess glucocorticoids. It has been suggested that regional stimulation of LPL might contribute to redistribution of fat by cortisol, analogous to its role in protein metabolism. Fat may be broken down in one region but stored in another under conditions of excess glucocorticoid availability: while lipolysis supplies increased amounts of FFA to peripheral tissue, circulating triglycerides (VLDL and chylomicrons), derived from increased food intake, may be preferentially stored in truncal fat (see below).

The increased FFA supply stimulates fatty acid oxidation, as shown both by indirect calorimetric measurements (decrease in RQ) (193) and appearance of ketone bodies (200). Whether cortisol also increases fat oxidation via direct intracellular effects, is unclear. 
Glucose levels are almost invariably reported to increase, both during acute and chronic hypercortisolemia, which is only partially attenuated by simultaneously increasing insulin levels $(201,207,208)$.

The decrease in insulin stimulated glucose uptake $(135,193,200)$ in response to hypercortisolism might be due to the increased circulating FFA. In chronic corticosteroid exposure changes in muscle fiber types might add to the insulin resistance (see above). As expected, whole body glucose oxidation diminishes (193). Glucose overproduction can be in part attributed to increased gluconeogenesis, which is induced by the increased supply of amino acids (especially alanine) and glycerol, derived from protein and fat mobilization $(135,208,209)$.

It is likely that the combination of increased circulating glucose and peripheral insulin resistance also reflects redistribution of fuel: peripheral organs are forced to derive their energy from fat, perhaps sparing glucose for use by the brain.

\section{Regional fat storage}

The central fat accumulation characteristic of chronic hypercortisolemia is likely due to regional differences in cortisol effects on either the adipocyte fatty acid uptake and/or lipolysis. Alternatively, regional differences in fat cell proliferation, differentiation or apoptosis in response to excess cortisol could play a role in the fat redistribution of Cushing's syndrome. Cortisol affects abdominal adipose tissue more than gluteo-femoral adipose tissue. In women with Cushing's syndrome, subcutaneous abdominal adipose tissue LPL activity increases threefold, whereas lipolytic rates were $50 \%$ less than those found in lean controls and lower body obese women (199). In contrast, in the femoral region lipolytic rates and LPL activity are similar in these three groups and in UBO women.

The net response of intra-abdominal adipose tissue to cortisol is similar to subcutaneous abdominal fat; treatment of Cushing's syndrome in women results in a clear re-distribution of fat from the visceral depots to the legs (205).

The physiological mediator of the regional difference in fat accumulation in response to excess cortisol has not been definitely determined It has been shown that the density of glucocorticoid receptors in visceral adipose tissue is higher than in other tissues (Rebuffé-Scrive in (205)), which could contribute to the observed effects. Unfortunately, the analyses of these studies are complicated by the lack of information given regarding the site from which fat was taken. When the data is reported, many times different sites are pooled for analysis, making it impossible to draw firm conclusions.

\section{B. The hypothalamic-pituitary-adrenal axis and obesity}

Visceral obesity shares many characteristics with Cushing's syndrome. Both conditions are associated with large abdominal subcutaneous fat cells with high lipolytic qualities, insulin resistance, hypertension and even buccal fat accumulation $(89,90)$. In addition, the changes in muscle fibers described in women with Cushing's syndrome are also found in UBO (199).

As neither of these is typical of lower body obesity, it has been suggested that visceral adiposity might be associated with changes in the HPA axis or cortisol receptors.

Although one glucocorticoid receptor polymorphism is associated with visceral obesity (210), it is unclear how common or physiologically relevant these types of gene polymorphisms are in visceral obesity.

Several changes in the HPA axis have been found in obesity, more so and more prevalent in the visceral than in the gluteo-femoral type (88). Secretory rates of cortisol are elevated in most, but not all studies, even when corrected for body surface area, although plasma concentrations of cortisol remain normal or slightly lowered $(88,196)$. This is thought to be a result of increased plasma cortisol clearance rates. Indeed, many, but not all human studies report 
increased urinary cortisol excretion rates in obesity (89). In addition, the urinary excretion of $5 \alpha$-reductase cortisol metabolites is positively correlated with waist circumference in nonobese men and post-menopausal women, suggesting that fat accumulation in the upper body is associated with increased cortisol turnover (87).

To the extent that cortisol production is increased in upper body obesity (and this is not uniformly accepted), there are indications that components of the HPA axis might be hyperactive. The adrenal sensitivity to ACTH, the responsiveness of ACTH and cortisol to $\mathrm{CRH}$ and several adrenal stimulation tests, such as postprandial HPA activation, have all been reported to be increased in obesity (89). However, all these parameters have also been found to be unchanged in other investigations; in addition, most studies show comparable ACTH levels in obese and lean individuals (89).

Thus, in obesity, the HPA axis is reported to be hyperactive or normal. This somewhat contradicts the concept of obesity being a CRH deficient state. This theory, which has been substantiated by rat- and molecular experiments, has further been challenged by the finding of normal to only slightly lowered cerebrospinal CRH levels in obese humans (89).

The study of the role of the HPA axis in visceral obesity is complicated by several obstacles, which likely account for some of the inconsistent findings. For example, earlier investigations studied different obesity phenotypes as one entity. This may have confounded the results considerably, as central obesity with its cushingoid fat distribution might be associated with entirely different dynamics of the HPA axis than peripheral obesity. Second, the regulation of the HPA axis is extremely complex in itself (see above) and is influenced by many heterogeneous and often uncontrollable variables, complicating standardization for research purposes and subsequently interpretation of the results.

One of these is the intertwined relationship between food intake, energy balance and the HPA axis. Ideally, one would like to know whether the hormonal abnormalities precede the energy surplus state, and whether introduction, removal and reintroduction of obesity consistently affect the different components of the HPA axis. This could, additionally, convincingly distinguish cause from result.

However, changes in body weight trigger counter-regulatory mechanisms aiming to maintain the steady state, which are thought to be mediated by the HPA axis. Thus compensatory mechanisms blur the effect of weight change, which is reflected in the fact that both overfeeding and starvation experiments have been found to amplify cortisol production (88).

In summary, an intertwined and complex relationship exists between energy balance, food intake and several components of the HPA axis. In visceral (and, to a much lesser extent, in gluteo-femoral) obesity, increased cortisol secretion and clearance rates result in normal or decreased plasma concentrations. There is no evidence of an increased exposure of cells and tissues to cortisol in visceral obesity, despite its similarities with Cushing's syndrome. Whether changes on intracellular or receptor level are involved is currently unknown.

\section{Managing glucocorticoid therapy in obese patients}

Considering the adverse effects of corticosteriods on muscle mass, body fat and insulin sensitivity, counteracting their side effects in long-term therapeutic use would be beneficial. This is particularly relevant in obese patients. One approach has been to co-administer proteinanabolic hormones together with corticosteriods; this has met with limited success only. GH has been shown to counteract the whole body protein catabolic effects of acute hypercortisolemia, but the aggravation of glucose intolerance precludes its therapeutic use (201). Testosterone therapy after long-term steroid treatment in asthma patients, slightly increased FFM $(0.9 \mathrm{~kg})$, decreased body fat and improved bone density (211). However, HDL levels also de- 
creased and it is unclear whether these and other, perhaps not yet discovered side effects outweigh the minor improvements in body composition.

Therefore, the preferred approach to maintain skeletal muscle mass and limit fat gain during corticosteroid therapy is physical activity and resistance exercise, especially considering its additional beneficial effects on reducing fat mass.

\subsubsection{Conclusions}

Growth Hormone, testosterone, insulin, corticosteroids and, to a lesser extent, estrogens, have a distinct effect on nutrient partitioning. Since the former two particularly influence fat distribution, they have been used in experimental therapy for centripetal obesity. This shall be further discussed in Chapter 4. 


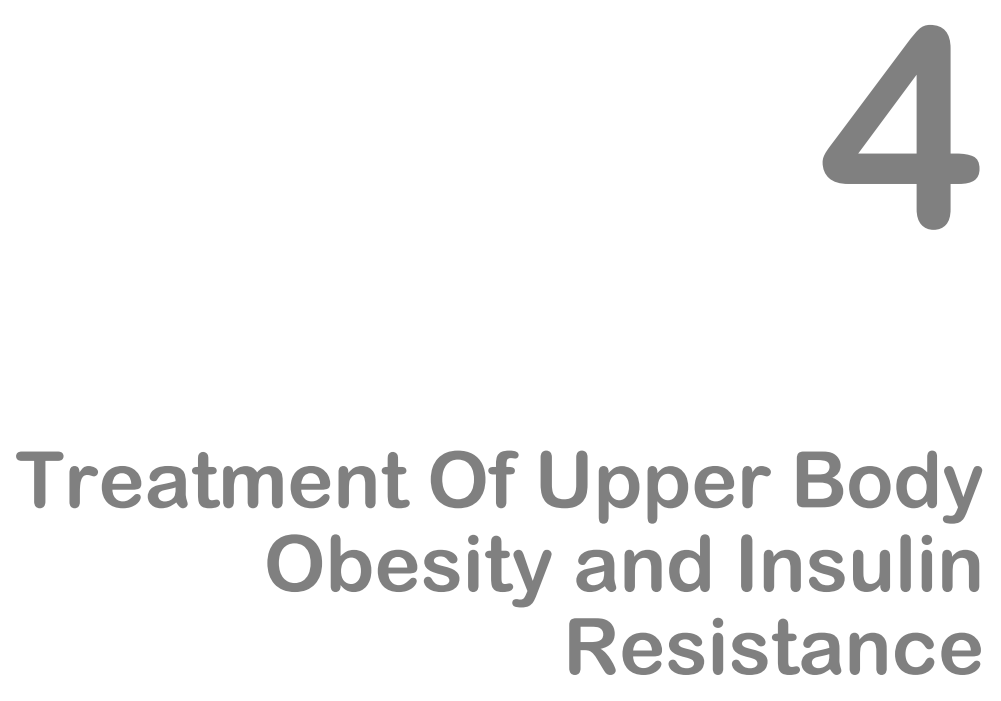




\section{$4 \quad$ Treatment Of Upper Body Obesity and Insulin Resistance}

$\begin{array}{lll}4.1 & \text { Introduction } & 77\end{array}$

4.2 Testosterone Administration $\quad 78$

From: 'Endocrine Control of Nutrient Partitioning'; Book chapter for 'Obesity:

Clinical Evaluation and Treatment', edited by RH. Eckel; LWW, Feb 2003

\subsection{Effects of Growth Hormone Administration in Human}

Obesity

Obesity Research 2003; 11:170-175

\subsection{Insulin Sensitization}

4.4.1 Energy Restriction and Physical Activity Training 86

4.4.2 Pharmacological Insulin Sensitizers 86

4.4.2.1 Characteristics of Pharmacological Insulin Sensitizers 86

4.4.2.2 Mechanism of Action of Thiazolidinediones $\quad 87$

4.4.2.3 Additional and Adverse Effects of Thiazolidinediones 88

4.4.3 Effect of Pioglitazone on Biochemical Indices of Non-Alcoholic Fatty Liver Disease in Upper Body Obesity

Clinical Gastroenterology and Hepatology, Sept '03;1(5): 384-7

4.5 Pioglitazone Treatment Contrasted to Diet and Exercise 94

4.5.1 Aims of Comparison 94

4.5.2 Methods of Research $\quad 94$

$\begin{array}{lll}\text { 4.5.2.1 Subjects } & 94\end{array}$

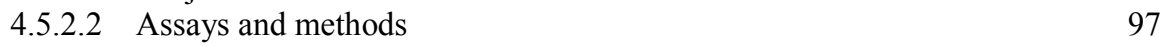

4.5.2.3 Principles and Calculations of Assays $\quad 99$

$\begin{array}{lll}\text { 4.5.2.4 Statistical analyses } & 102\end{array}$

4.5.3 Pioglitazone Increases Non-Esterified Fatty Acid Clearance in Upper Body Obesity

Diabetologia. 2006 Jan;49(1):149-57. Epub 2005 Dec 2

4.5.4 Diet/Exercise versus Pioglitazone: Effects of Insulin Sensitization with Decreasing or Increasing Fat Mass on Adipokines and Inflammatory Markers JCEM 2006 Sep;91(9):3418-25. Epub 2006 Jun 27

4.5.5 Treatment of Obesity with Diet/Exercise vs. Pioglitazone has Distinct Effects on Lipoprotein Particle Size

Atherosclerosis 2006 Oct;188(2):370-6. Epub 2005 Nov 28

4.5.6 Effects of Pioglitazone Versus Diet and Exercise on Metabolic Health and Fat Distribution in Upper Body Obesity

Diabetes Care 26:3148-3152, 2003 


\subsection{Introduction}

The growing prevalence of obesity and its consequences worldwide warrant large-scale prevention and treatment (see Chapter 1). Treatment may be either macro-environmental (large scale interventions promoting physical activity and improving the quality of food intake), micro-environmental, (changes in an individual's environment), medical (diet/exercise, behavior modification, medication), or invasive (e.g. bariatric surgery, gastric pacing, intra-gastric balloons). We shall focus on medical options only.

Energy restriction and exercise training (diet and exercise (Diet/ex)) can be considered the gold standard for the treatment of obesity. Pharmacological treatment of obesity includes intestinal fat uptake inhibitors (orlistat) and appetite reducing agents, affecting either serotonin and norepinephrine reuptake (sibutramine) or cannabinoid receptors (rimonabant) (212). These drugs are generally more successful if combined with Diet/ex.

Other conservative options are directed towards reducing the consequences of overweight (mainly insulin resistance) rather than the weight itself. Hormonal treatment, such as the administration of testosterone or growth hormone, is suggested by some to attenuate the consequences of insulin resistance; this, however, is controversial and shall be discussed in Chapters 4.2 and 4.3. Insulin sensitizing drugs include biguanides (metformin) and thiazolidinediones. Both are often used in clinical practice, but their mechanisms of action are not entirely understood, and it is largely unclear to what extent they are qualitatively and quantitatively comparable to Diet/ex.

In Chapters 4.4 onwards, we shall discuss thiazolidinedione treatment and contrast its effects to those of Diet/ex regarding quantitative and pathophysiological aspects of insulin resistance. Their respective effects on fatty acid trafficking (4.5.3), adipokine secretion (4.5.4), lipoprotein particle size (4.5.5) and on body composition and adipose tissue remodeling (4.5.6) shall be evaluated. 


\title{
4.2 Testosterone Administration In Upper Body Obesity
}

\author{
From: S Shadid and MD Jensen 'Endocrine Control of Nutrient Partitioning'; \\ Book chapter for 'Obesity: Clinical Evaluation and Treatment', edited by \\ Robert H. Eckel, MD. Lippincott, Williams and Wilkins, Feb 2003
}

Given the known effects of testosterone on nutrient partitioning (Chapter 3.2.1) and the finding that visceral obesity in men is associated with reduced testosterone, it would be logical to consider testosterone administration as a treatment option for obese men. A number of studies have examined this approach: intramuscular $(250-500 \mathrm{mg})$, oral $(4 \mathrm{x} 40 \mathrm{mg})$ and transdermal $(125-250 \mathrm{mg} /$ day) testosterone administration consistently caused a significant decrease in visceral fat compared to placebo $(160,161,213,214)$. Somewhat surprisingly, however, no significant changes were observed in total body fat quantities or lean body mass.

Testosterone administration also resulted in a reduction in waist-hip ratio, cholesterol $(161,214)$ and, in some studies, serum triglyceride concentrations (214). In addition, it improved insulin action with respect to glucose disposal $(161,213,214)$. The latter was most pronounced in the men with the lowest testosterone levels prior to the intervention and is thought to be an indirect effect of the reducing visceral fat mass (see above). Dihydrotestosterone administration had neither of these effects $(213,214)$; neither did the analogous (214)lowering of androgen concentrations in hyperandrogenic women using $\mathrm{GnRH}$ analogs (which also reduce serum estrogen) (215).

Nevertheless, whether testosterone should be administered to obese men is open to debate. The precise explanation for low free testosterone concentrations in obese men is unknown. Analogous to GH, it may be that the low testosterone results from adaptations to an altered environment rather than representing a primary endocrine problem causing visceral obesity. This is confirmed by the fact that weight loss reverses the abnormalities. For example, replacement of testosterone has been reported to create sleep apnea in men (216) and excess endogenous testosterone is associated with sleep apnea in women (217).

In summary, testosterone influences protein and fat deposition and has major effects on regional adipose tissue depots. There are indications that testosterone replacement may have beneficial effects on fat distribution, lipid profiles and insulin sensitivity in viscerally obese men with low serum testosterone concentrations. Routine treatment, except in the case of true hypogonadism however, is still controversial. Whether the benefits of testosterone replacement in men with modest reductions in serum concentrations outweigh the risks of treatment is currently unknown. 


\title{
4.3 Effects of Growth Hormone Administration in Human Obesity
}

S Shadid and MD Jensen; Obesity Research 2003; 11:170-175

\begin{abstract}
Objective: To summarize the reports in the literature regarding the effect of growth hormone (GH) treatment of obesity.

Purpose of Study: To determine the beneficial vs. adverse reported effects of exogenous GH administration to obese adults.

Research Methods and Procedures: Clinical trials of GH treatment of obese adults were reviewed and summarized. Specifically, information regarding the effects of GH on body fat and body fat distribution, glucose tolerance/insulin resistance, and adverse consequences of treatment were recorded.

Results: GH administered together with hypocaloric diets did not enhance fat loss nor preserve lean tissue mass. No studies provided strong evidence for an independent beneficial effect of GH on visceral adiposity. In all but one study glucose tolerance during GH treatment suffered relative to placebo.

Conclusion: The bulk of studies indicate little or no beneficial effects of GH treatment of obesity despite the low serum GH concentrations associated with obesity.
\end{abstract}




\section{Introduction}

Obesity is associated with a number of endocrine and metabolic abnormalities. These include, but are not limited to, insulin resistance (which is correlated with visceral adiposity) and decreased serum growth hormone (GH) concentrations. The mechanism of the low GH in obesity is not understood, nor is it clear whether the relationship with visceral obesity is causal. Nevertheless, the beneficial effects of GH on lipolysis and on fat distribution found in patients with GH deficiency have led to experimental supplementation of this hormone in viscerally obese patients. These studies have tested the hypothesis that low levels of GH contribute to central obesity and related metabolic abnormalities. The predicted results are reductions in intra-abdominal fat and improved metabolic health.

We reviewed the results of 16 published studies on GH administration in (predominantly central) obesity, found via MEDLINE searches. This included a large study of overweight elderly adults with high waist-to-hip ratios (218).

A number of the authors provided optimistic comments on the results of GH treatment $(86,151,152,219)$. Because details of the reported effects of $\mathrm{GH}$ administration were commonly at odds with the favorable conclusions, we elected to summarize the treatment trials to look for consistent findings.

A brief overview of the current knowledge of GH physiology and the pathophysiology of hyposomatotropinism in obesity are first provided in order to put the treatment trial results in perspective.

\section{Growth Hormone and Nutrient Partitioning}

In addition to the induction of growth, an important function of GH is the regulation of nutrient partitioning. $\mathrm{GH}$ enhances the oxidation of fatty acids relative to glucose or amino acids (119). This is achieved by increasing $(117,118,120)$ adipose tissue lipolysis and/or reducing triglyceride storage in a non-uniform manner such as to redistribute adipose tissue from intraabdominal to peripheral depots in addition to decreasing body fat mass.

Furthermore, GH has protein anabolic and diabetogenic effects. The latter arise from the (direct or indirect) ability of GH to enhance endogenous glucose production and to inhibit cellular glucose uptake, thus raising plasma glucose concentrations. Prolonged excess GH can lead to pancreatic $\beta$-cell failure such that insulin secretion cannot overcome the insulin resistance resulting in hyperglycemia and eventually diabetes. Another common adverse effect of excess $\mathrm{GH}$ is an abnormal expansion of extracellular fluid.

Many GH effects, such as its effects on growth and protein synthesis are mediated via insulinlike growth factors (IGF), mainly IGF-1; GH largely mediates the production of IGF-1. An exception is the GH effects on fat metabolism, which are not IGF-1 dependent $(119,125)$. IGF-1 exerts feedback inhibition of GH secretion at the hypothalamic and pituitary levels.

\section{Growth Hormone in Obesity}

The role of GH in obesity is complex and somewhat controversial. Although primary growth hormone deficiency leads to centripetal adiposity, visceral obesity per se also results in a secondary reduction in serum GH concentrations (86).

The difference in the pathophysiology of the two conditions is primarily reflected by the disparate IGF-1 responses. IGF-1 is very low in primary GH deficiency, but may be normal, high 
or modestly reduced in obesity $(86,143,143)$. In the latter case, a simultaneous change in the availability of IGF-binding proteins (decreased IGFBP 1 and 2, as well as increased IGFBP 3) usually results in normal to elevated free, biologically active, IGF-1, equaling or exceeding those in lean subjects (86). This might explain the fact that IGF-dependent functions of GH, such as the growth ability of obese children, remain unchanged (86). Successful weight loss is reported to improve or normalize GH parameters (86), strongly suggesting this is a secondary, not primary phenomenon.

Nevertheless, the reasons for the hyposomatotropinism in obesity and its mechanisms have yet to be clarified. Reductions in spontaneous GH secretion (as much as $6 \%$ for each unit increase in BMI (220)) and in the half-life of circulating GH (221) have been reported. Moreover, the GH response to pharmacological (GHRH, L-Dopa) and physiological stimuli, such as sleep, physical exercise, insulin-induced hypoglycemia, and corticosteroids is impaired in obesity (86).

Some of the theories on the cause of altered GH physiology in obesity involve the increased concentrations of leptin, insulin, FFA and IGF-1. Conflicting reports on the effect of leptin have been published, however, with in vitro and in vivo studies finding both stimulation and inhibition of GH release (86).

IGF-1 and FFA are thought to contribute to the hyposomatotropinism via feedback inhibition of GH secretion; spontaneous and stimulated GH release increases in obese subjects after administration of acipimox, a nicotinic acid analogue that lowers FFA concentrations (86). The quantitative contribution of elevated FFA concentrations to altered GH physiology in obesity, however, remains unclear.

\section{Growth Hormone Administration In Obesity}

The low GH concentrations in visceral obesity have led to experimental administration of this hormone in obese subjects. Its lipolytic effects were expected to induce weight loss, and its protein anabolic effects to protect against the negative nitrogen balance often accompanying hypocaloric diets. In addition, GH's fat-redistributing qualities were predicted to reduce visceral fat and thus to improve metabolic health, analogously to the insulin sensitizing effects seen after the reduction of intra-abdominal adipose tissue by exercise, diet or surgery. $(144,145,146,147,148,149,150,151,152,153)$.

The rationale for these expectations is supported by the finding that GH replacement therapy in patients with primary GH deficiency increases abnormally low muscle mass and redistributes intra-abdominal fat towards peripheral depots $(118,119,121,122,123)$. We found little or no evidence that the goals of favorable fat redistribution or metabolic improvement were achieved by GH supplementation of obese subjects, however (table 4.3.1 and 4.3.2).

\section{Effects on body composition}

The subcutaneous administration of recombinant human $(r h) \mathrm{GH}$ to obese volunteers in combination with an energy-restricted diet has not been found to result in a greater decrease in fat mass or preservation of lean tissue when compared with diet alone $(117,151,153,219,222)$. Only when given in the context of an isoenergetic diet has GH been reported to decrease total body fat relative to placebo, although the differences were relatively minor $(144,152)$.

In this context it is critical to understand that the methods commonly used to assess body composition (DEXA, underwater weighing, BIA) have serious limitations when applied to conditions in which extracellular fluid shifts can occur. Each of these techniques measures FFM, a component of which is extracellular fluid. These approaches therefore cannot distin- 
guish changes in FFM due to changes in body cell mass as opposed to changes in extracellular fluid. This limits the interpretation of FFM as a surrogate measurement for lean body mass/body cell mass (which is therefore confounded by GH-induced water retention) and therefore percent body fat. If weight increases by fluid retention, percent body fat will decrease even if total body fat does not change.

This could well explain why some authors (151) report similar fat and weight loss in GH vs. placebo, but claim a significant difference in fat loss as a fraction of weight change.

Table 4.3.1. Effect of Growth Hormone administration in obesity on body composition

\begin{tabular}{|c|c|c|c|c|c|c|c|}
\hline Authors & $\mathbf{n}$ & $\begin{array}{l}\text { Mean } \\
\text { daily dose }\end{array}$ & \# wks & Diet & Weight loss $^{1}$ & $\begin{array}{l}\text { Body comp } \\
\text { method }\end{array}$ & Fat loss \\
\hline Clemmons (222) & 8 & $50 \mu \mathrm{g} / \mathrm{kg}$ IBW & $11^{2}$ & $\mathrm{H}$ & $4 \%$ & UWW & Similar $(2.5 \%)$ \\
\hline $\begin{array}{l}\text { Drent } \\
(148)\end{array}$ & 15 & $1.9 \mathrm{mg}$ & 8 & $\mathrm{H} \dagger$ & $13.7 \%$ & BIA & Similar (14\%) \\
\hline Johansson (152) & 30 & $9.5 \mu \mathrm{g} / \mathrm{kg}$ & 40 & I & NR & Total body ${ }^{40} \mathrm{~K}^{+}$ & $\begin{array}{l}\mathrm{GH}(2.5 \%)>\mathrm{P} \\
(\equiv) \mathrm{S}\end{array}$ \\
\hline Kim (151) & 24 & $9.5 \mu \mathrm{g} / \mathrm{kg} \mathrm{IBW}$ & 12 & $\mathrm{H}$ & $9 \%$ & BIA & Similar $(4.5 \%)$ \\
\hline $\begin{array}{l}\text { Münzer } \\
(218)\end{array}$ & $110^{3}$ & $8.6 \mu \mathrm{g} / \mathrm{kg}$ & 26 & I & NR & \multicolumn{2}{|c|}{ Anthropo-metric NR } \\
\hline Nam (223) & $18^{4}$ & $7.7 \mu \mathrm{g} / \mathrm{kg}$ & 12 & $\mathrm{H}$ & $6 \%$ & BIA & $\begin{array}{l}\mathrm{GH}(7 \%)>\mathrm{P} \\
(4.5 \%) \mathrm{S}\end{array}$ \\
\hline Norrelund (219) & 15 & $18.2 \mu \mathrm{g} / \mathrm{kg} \mathrm{IBW}^{5}$ & 4 & $\mathrm{H}$ & $4.5 \%$ & DEXA & NR \\
\hline Richelsen (144) & $9 *$ & $30 \mu \mathrm{g} / \mathrm{kg} \mathrm{IBW}$ & 5 & I & \multicolumn{2}{|c|}{$\begin{array}{l}-2 \%(\mathrm{GH}) ; 0 \% \mathrm{DEXA} \\
(\mathrm{P})\end{array}$} & $\mathrm{GH}(2.5 \%)>\mathrm{P}(\equiv)$ \\
\hline Richelsen (117) & 18 & $13.3 \mu \mathrm{g} / \mathrm{kg}$ & 4 & $\mathrm{H}$ & $4.5 \%$ & DEXA & Similar (8\%) \\
\hline $\begin{array}{l}\text { Skaggs } \\
(150)\end{array}$ & $12^{6}$ & $80 \mu \mathrm{g} / \mathrm{kg}$ IBW & 4 & I & \multicolumn{2}{|c|}{$-1 \%(\mathrm{GH}) ; \equiv \mathrm{PUWW}$} & $\mathrm{GH}(2 \%)>\mathrm{P}(\equiv)$ \\
\hline $\begin{array}{l}\text { Snyder } \\
(149)\end{array}$ & 8 & $100 \mu \mathrm{g} / \mathrm{kgIBW}$ & $15^{7}$ & $\mathrm{H}$ & $7.5 \%$ & UWW & Similar (4.1 \%) \\
\hline $\begin{array}{l}\text { Snyder } \\
(147)\end{array}$ & $11^{*}$ & $50 \mu \mathrm{g} / \mathrm{kgIBW}^{8}$ & 5.5 & $\mathrm{H}$ & $\begin{array}{l}7.3(\mathrm{GH}) \mathrm{vs} . \\
8.4 \mathrm{~kg}^{9} \mathrm{~S}\end{array}$ & UWW & Similar $(2.7 \%)$ \\
\hline $\begin{array}{l}\text { Snyder } \\
(146)\end{array}$ & $20^{*}$ & $50 \mu \mathrm{g} / \mathrm{kg} \mathrm{IBW}$ & 10 & $\mathrm{H}$ & $14 \mathrm{~kg}^{\mathrm{ix}}$ & UWW & Similar $(8 \%)$ \\
\hline $\begin{array}{l}\text { Snyder } \\
(224)\end{array}$ & $11^{*}$ & $50 \mu \mathrm{g} / \mathrm{kg} \mathrm{IBW}$ & 10 & $\mathrm{H}^{10}$ & $8 \mathrm{~kg}^{\mathrm{ix}}$ & UWW & $\begin{array}{l}\mathrm{GH}(3.7 \%)^{11}>\mathrm{P} \\
(2.8 \%) \mathrm{S}\end{array}$ \\
\hline Tagliaferi (145) & 20 & $46.6 \mu \mathrm{g} / \mathrm{kg} \mathrm{IBW}$ & 4 & $\mathrm{H}$ & $6 \%$ & DEXA & Similar $(9.5 \%)$ \\
\hline Thompson (153) & $33^{12}$ & $25 \mu \mathrm{g} / \mathrm{kg}$ & 12 & $\mathrm{H} \dagger$ & $3.5 \%$ & DEXA & Similar (16\%) \\
\hline
\end{tabular}

Studies were placebo controlled unless indicated otherwise. 'Similar' refers to GH vs. placebo.

Abbreviations: BIA: bio-electric impedance analysis; CT: computed tomography; comp= composition; GH: growth hormone; IBW: ideal body weight; NR: not reported; S: significant; P: placebo; UWW=under water weighing; wks=weeks; $\mathrm{I}=$ isocaloric; $\mathrm{H}=$ hypocaloric $\equiv$ no change; * placebo cross-over; $\dagger$ plus exercise

\footnotetext{
${ }^{1}$ Weight loss similar in $\mathrm{GH}$ and placebo in all studies unless listed otherwise

${ }^{2} \mathrm{GH}$ given from week 3-5 or 8-10 only

34 groups: GH, HRT, GH+ HRT, plac; all subjects $>65$ years old

${ }^{4}$ type II diabetic subjects; mean $\mathrm{BMI}=28$

${ }^{5}$ Gradual build up

${ }^{6}$ baseline weight of GH group is $18 \mathrm{kgs}$ higher than placebo!

$72 \times 4$ weeks of which GH or plac

8 only GH for 28 days; rest: diet only

9 initial weight NR;

${ }_{11}^{10}$ high carbohydrate vs. high fat diet; parameters mentioned in this table were similar

11 inter-individual differences ++

124 groups: GH, IGF, GH + IGF, plac; least weight loss in GH group, most in IGF+GH group (6\%)
} 
These limitations do not apply to measurement of total body potassium as a measure of lean body mass. The only study taking advantage of this body composition measurement technique found no difference between $\mathrm{GH}$ and placebo treatment (152). One group reported an increase in FFM when GH was combined with an isoenergetic diet, however this finding is open to interpretation given that the investigator used DEXA as the means of body composition measurement (144).

Changes in muscle mass/lean tissue can also be estimated by assessing nitrogen balance and muscle strength. Muscle strength was reported to increase equally in obese adults treated with GH vs. placebo treated with a hypocaloric diet and an exercise program (153). Nitrogen balance has been found to be more negative in the placebo treated than GH treated obese volunteers treated with energy restricted diets $(145,146,148,151,222,224)$. This difference, however, was attenuated or lost after four to five weeks of treatment in some studies $(146,147)$. We view this as a considerable limitation in light of the short duration and the lack of longterm follow up in most studies.

\section{Effects on fat distribution}

All four studies that used CT or MRI to assess changes in fat distribution in response to GH treatment reported decreases in intra-abdominal fat of 7-18\% $(144,152,152,218,218,223,223)$. Münzer et al, authors of the largest study (111 volunteers) (218), found that elderly men, but not women, had a statistically significant (3.9\%) loss of visceral fat.

Interpreting this finding by itself is impossible because almost any intervention resulting in body fat loss also results in a disproportionate loss of visceral fat compared with subcutaneous fat.

To confidently assess whether GH treatment specifically enhances visceral fat loss one must match GH treated groups with placebo treated groups with equal fat loss. Unfortunately, most studies in which placebo treated volunteers lost equal amounts of fat compared with GH treated volunteers did not assess regional fat loss. The one study that did report these results (151) found similar visceral fat loss in placebo and GH treated groups. We conclude that the observed fat redistribution reported in some studies cannot be confidently be attributed to GH administration.

\section{Metabolic effects}

If $r h \mathrm{GH}$ therapy preferentially reduced visceral fat, would the expected improvements in metabolic health appear? Apparently not. When reported, insulin resistance worsens, plasma FFA concentrations increase, and serum HDL-cholesterol concentrations (117) decrease. Despite reassuring statements, in all studies we reviewed except one (223) even small amounts of GH decreased insulin stimulated glucose disposal rates and/or increased levels of glucose, insulin, C-peptide and 24 hour urinary C-peptide excretion $(117,144,145,146,147,148,152,219,223)$. This was especially prominent, but not limited to, studies in which $r h \mathrm{GH}$ was combined with an iso-energetic diet. In several studies the adverse effects on glucose metabolism were either not reported $(150,150,153,218)$, or did not reach statistical significance. In other studies insulin sensitivity was claimed to remain unchanged or even to improve, based on criteria that do not describe the full picture.

For example, Johansson et al show similar glucose disposal rates in their figure 3 (152), glucose and insulin concentrations at the end of 9 months of GH versus placebo treatment, but marked worsening in the GH group in all time points measured in between. This is not a reassuring result. 
Table 4.3.2. Metabolic effects of Growth Hormone administration in obesity. Studies were placebo controlled unless indicated otherwise

\begin{tabular}{|c|c|c|c|c|c|c|c|c|c|c|}
\hline \multirow[t]{2}{*}{ Authors } & \multirow[t]{2}{*}{$\mathbf{N}$} & \multirow[t]{2}{*}{ FFA } & \multicolumn{2}{|c|}{ F-Insulin } & \multicolumn{2}{|c|}{ F-Glucose } & \multicolumn{2}{|c|}{ F-Cpeptide } & \multirow[t]{2}{*}{ Miscellaneous } & \multirow[t]{2}{*}{ Complications } \\
\hline & & & GH & $\mathbf{P}$ & GH & $\mathbf{P}$ & GH & $\mathbf{P}$ & & \\
\hline $\begin{array}{l}\text { Clemmons } \\
(222)\end{array}$ & 8 & NR & $\equiv$ & & $\equiv$ & & NR & & No glucosuria & Mild Edema $62 \%$ \\
\hline Drent (148) & 15 & NR & $\mathrm{GH}>$ & & $\mathrm{GH}=1$ & & NR & & $\begin{array}{l}\text { GH: Glucagon } \uparrow \\
(\ddagger \text { cf } \mathrm{P})\end{array}$ & $\begin{array}{l}\uparrow \mathrm{BP}(\mathrm{GH})^{1} \text {; other- } \\
\text { wise NR }\end{array}$ \\
\hline $\begin{array}{l}\text { Johansson } \\
(152)\end{array}$ & 30 & $\equiv$ & $\uparrow$ & $\equiv$ & $\equiv$ & $\equiv$ & $\uparrow$ & $\equiv$ & $\downarrow \mathrm{GDR}^{2}$ & $\begin{array}{l}\text { CTS 3\%; FR 27\%; } \\
\text { Muscle stiffness } 6 \%^{3} \text {; }\end{array}$ \\
\hline $\operatorname{Kim}(151)$ & 24 & $\downarrow^{4}$ & NR & & NR & & NR & & $\begin{array}{l}\text { Insulin AUC dur- } \\
\text { ing OGTT } \downarrow, \mathrm{P}> \\
\text { GH }\end{array}$ & Edema 13\% \\
\hline Münzer (218) & 110 & & NR & & NR & & NR & & & CTS, arthralgia \\
\hline Nam (223) & 18 & $\downarrow \dagger$ & $\downarrow$ & $\downarrow$ & $\downarrow$ & $\downarrow$ & NR & & $\mathrm{GDR} \uparrow(\mathrm{GH})$ & 3 edema, 2 arthralgia \\
\hline $\begin{array}{l}\text { Norrelund } \\
(219)\end{array}$ & 15 & $\uparrow \uparrow$ & $\uparrow$ & $\downarrow$ & $\equiv$ & $\equiv$ & $\uparrow \ddagger$ & $\downarrow \ddagger$ & $\begin{array}{l}\downarrow \text { gluc turnover \& } \\
\text { oxidation } \dagger\end{array}$ & NR \\
\hline Skaggs (150) & 12 & NR & NR & & NR & & NR & & & NR \\
\hline $\begin{array}{l}\text { Richelsen } \\
\text { (144) }\end{array}$ & $9 *$ & $\uparrow$ & $\uparrow \uparrow$ & $\S$ & $\uparrow$ & $\S$ & $\uparrow$ & $\S$ & & CTS 5/9; edema \\
\hline $\begin{array}{l}\text { Richelsen } \\
(117)\end{array}$ & 18 & $\uparrow \dagger$ & $\uparrow$ & $\downarrow$ & NR & & & & & NR \\
\hline Snyder (149) & 8 & $\uparrow^{5}$ & $\mathrm{GH}>>$ & & $\mathrm{GH} \gg>$ & & $\mathrm{GH}>$ & & $\begin{array}{l}\mathrm{GH}: \uparrow \mathrm{Cp} \text { excre- } \\
\text { tion; } \mathrm{Cp} \text {, glu }+ \text { ins } \\
>\mathrm{P}\end{array}$ & NR \\
\hline Snyder (147) & $11 *$ & Similar & $\mathrm{GH} \gg>$ & & $\mathrm{GH}>\mathrm{I}$ & & $\mathrm{GH}>$ & & & Fluid retention \\
\hline Snyder (146) & $20 *$ & $\begin{array}{l}\mathrm{GH}> \\
\mathrm{P}\end{array}$ & $\mathrm{GH}=$ & & $\mathrm{GH}>\mathrm{I}$ & & NR & & $\begin{array}{l}\mathrm{Cp} \text { excretion } \mathrm{GH}= \\
\mathrm{P}\end{array}$ & FR, edema \\
\hline Snyder (224) & $11 *$ & $\uparrow$ & $\equiv$ & $\S$ & $\uparrow^{7}$ & $\S$ & NR & & $\begin{array}{l}\text { Cp excretion } \mathrm{GH}> \\
\mathrm{P}^{\mathrm{vi}}\end{array}$ & FR; edema \\
\hline $\begin{array}{l}\text { Thompson } \\
\text { (153) }\end{array}$ & 33 & NR & NR & & & & & & & $\begin{array}{l}89 \% \text { edema }^{8} \text {; hand } \\
\text { numbness } 44 \% \text {; fa- } \\
\text { tigue } 56 \%\end{array}$ \\
\hline $\begin{array}{l}\text { Tagliaferi } \\
(145)\end{array}$ & 20 & $\uparrow \uparrow$ & $\uparrow$ & $\downarrow$ & $\equiv$ & $\equiv$ & NR & & & $\mathrm{NR}$ \\
\hline
\end{tabular}

BP: blood pressure; Cp: C-peptide; CTS: carpal tunnel syndrome; F: fasted; FR: fluid retention; GDR: glucose disposal rate; GH: growth hormone; glu: glucose; ins: insulin; NR: not reported; pp: post-prandial. Symbols: $\equiv$ no change * placebo cross over; $\dagger \mathrm{GH}$ and placebo equally; $\$$ non-significant; $\S$ initial placebo values are NR; post $\mathrm{GH}$ values are higher than placebo

Nam et al (223) reported an improvement of insulin action and fat distribution in a 12-week placebo-controlled study of 18 Type 2 diabetic volunteers. Nevertheless, some potential limitations of this study should be considered. Body fat and lean tissue was assessed using bioelectrical impedance analysis (BIA), a method notoriously sensitive to changes in extracellular fluid.

\footnotetext{
${ }^{1}$ despite weight loss/exercise

2 except at final time point

${ }^{3}$ these caused average dose reduction of $0.17 \mathrm{mg} /$ day

${ }^{4}$ AUC during OGTT; decreases more than in placebo; fasted not mentioned but is higher than placebo too

${ }^{5} 4$ hrs after GH injection; fasted was unchanged

${ }^{6}$ quantity unclear

${ }^{7}$ in high carbohydrate diet only

${ }^{8} 5 / 33$ dropped out (intolerable edema)
} 
The changes in lean body mass and visceral fat are not convincingly significant when the standard deviation and the sample size are considered. In addition, although visceral fat is reported to be more reduced in the GH group, the means of data analysis (visceral fat area change divided by body fat change) has not been validated as an appropriate index of visceral fat loss (see comments above).

Although glucose disposal was found to increase more and FFA to decrease more in GH treated patients, these results are discordant with all other above-mentioned studies. The results may relate to a unique study population or may represent a Type 1 statistical error. These results should be reproduced in larger, diverse populations before GH therapy could be considered for Type 2 diabetes mellitus.

In summary, the vast majority of the studies describing metabolic parameters show a clear trend towards metabolic deterioration with $\mathrm{GH}$ administration to adults with visceral obesity.

\section{Adverse effects}

In addition to the metabolic side effects, approximately $20-40 \%$ of the volunteers receiving GH developed fluid retention, arthralgias or carpal tunnel syndrome $(144,146,147,152,153)$. Furthermore, in children the induction of sleep apnea is reported (225).

\section{Conclusions}

In summary, there we found no evidence for metabolic benefits of $\mathrm{GH}$ administration in obesity in the absence of true GH deficiency. On the contrary, almost all studies reporting the effects of $\mathrm{GH}$ administration on glucose metabolism in obesity show trends towards worsening of insulin resistance. Thus, if visceral fat loss were truly achieved, its possible benefits (a primary incentive for GH administration) are lost; however, any demonstrable effects on body composition were minimal, not necessarily attributable to the GH administration, and only present when isocaloric diets were given. Although GH administration attenuated nitrogen loss, this effect was lost after a few weeks, whereas it did not convincingly affect other measures of lean body mass. Troublesome non-metabolic side effects of GH include fluid retention, arthralgia and carpal tunnel syndrome.

Perhaps not surprisingly, growth hormone returns to normal following weight reduction in obesity (86). This finding strongly suggests that low GH is a consequence, not a cause of central obesity. Considering the high costs and the lack of understanding of the long-term consequences of GH treatment of obesity we argue against its use for this purpose. 


\subsection{Insulin Sensitization}

\subsubsection{Energy Restriction and Physical Training}

Insulin sensitivity is inversely correlated with BMI (226). Even modest changes in weight (5$7.9 \mathrm{~kg}$ or $7 \%$ of initial body weight) after the age of 18 affect the relative risk of developing DM in otherwise healthy people in various weight categories, as shown, among others, by the Nurse's Health Study (226) and the Diabetes Prevention Trial (227). In the former, $5.0 \mathrm{~kg}$ or more weight loss reduced the risk of developing DM by $50 \%$, whereas weight gain increased this risk, even in low BMI categories.

Of note, physical activity benefits insulin sensitivity independently of weight loss or energy restriction. This is, among others, associated with increases in both mass and oxidative capacity of muscle, and with improved regulation of $\beta$-adrenergic adipose tissue lipolysis (228).

The main practical problems involved with Diet/ex are the time investment and the discipline required from health care providers and patients. In addition, the lost weight is easily regained when old habits are reintroduced and exposure to toxic environments such as modern Western societies continues. Therefore, the approach of nutritional and exercise aspects of weight loss as life style changes rather than as temporary diets is essential and has, indeed, been shown to have the best long-term results (229). Behavioral-therapeutic education on eating and exercise, known as 'behavior modification programs', are particularly helpful herein. An example of the latter is the LEARN program for Weight Management (230).

\subsubsection{Pharmacological Insulin Sensitizers}

\subsubsection{Characteristics of Pharmacological Insulin Sensitizers}

Pharmacological insulin sensitization can be achieved with biguanides (metformin) or thiazolidinediones (troglitazone, pioglitazone and rosiglitazone etc).

Until the introduction of thiazolidinediones (TZDs), biguanides were the only available oral insulin sensitizers. Metformin improves glycemic control primarily by increasing liver insulin sensitivity; this decreases hepatic glucose output and, perhaps indirectly, lowers plasma lipid concentrations. Its exact mechanism of action, however, remains unknown. Nonetheless, its value was demonstrated, both in reducing the incidence of diabetes in obese groups (the Diabetes Prevention Program (227) and the Indian Diabetes Prevention Program (231)) and in achieving glycemic control and prevention of vascular complications in overweight type 2 diabetic patients (the United Kingdom Prospective Diabetes Study (UKPDS) (232)).

TZDs have also proven their effectiveness on glycemic control as monotherapy or in combination with metformin or sulfonurea derivates (233-236). In a head-to-head comparison with metformin, pioglitazone (PIO) was demonstrated to achieve similar glycemic control, but better improvement of lipids and insulin resistance (234). 
More recently, PIO was also shown to reduce all-cause mortality, nonfatal myocardial infarction and stroke rates in a trial assessing its effect on cardiovascular and other clinical end points (PROactive trial (237)). Macrovascular complications were not statistically significantly affected $(\mathrm{p}=0.10)$. However, the

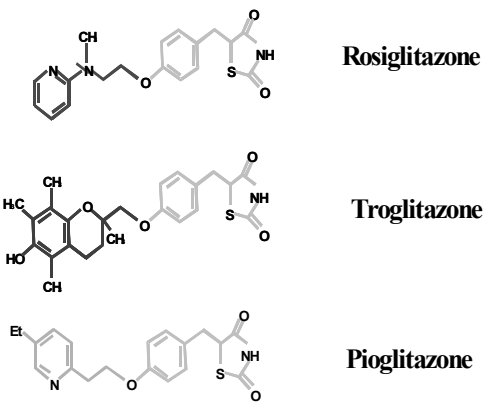

Figure 4.1 The thiazolidinediones short study interval and other concerns limited the interpretation of this study. This shall be further discussed in Chapter 5 .

Attempts to evaluate the effectiveness of TZDs in preventing diabetes have been delayed by the retraction of the prototype TZD, troglitazone (see below). Nevertheless, in the TRIPOD (Troglitazone in Prevention of Diabetes) study, 30 months of troglitazone treatment decreased average annual diabetes incidence rates from 12.1 (placebo) to $5.4 \%$ in Hispanic women with prior gestational diabetes (238). The sequel, the PIPOD study (Pioglitazone In Prevention Of Diabetes), confirmed that TZDs preserve pancreatic beta-cell function in

this study population, but did not provide evidence on a potential protective function of PIO in the development of DM 2, among others because it lacked a control group (239).

Since TZDs have a central role in the following chapters, we will discuss some aspects of their mechanisms of action more in depth.

\subsubsection{Mechanism of action of thiazolidinediones}

TZDs enhance insulin signaling by influencing peroxisome proliferator-activated receptor (PPARs, which are nuclear transcription factors) of the $\gamma$ - subtype (see below). Some, such as pioglitazone, also have intrinsic PPAR- $\alpha$ stimulatory qualities.

Pioglitazone (Actos $\left.{ }^{\circledR}\right)$, rosiglitazone (Avandia $\left.{ }^{\circledR}\right)$ and troglitazone (Rezulin ${ }^{\circledR}$ ) were most generally used in TZD research and clinical practice until the latter was retracted from the world market due to its potentially lethal side-effects (idiosyncratic liver failure; see 4.4.2.3). The three compounds are similar but differ, among others, in their relative PPAR- $\alpha$ and $-\gamma$ stimulatory effects: the strongest PPAR- $\gamma$ agonist, rosiglitazone, has no relevant $\alpha$-agonist action; in turn, pioglitazone has a weaker intrinsic $\gamma$-action than rosiglitazone, but profits from $\alpha$ and $\gamma$ synergism and, possibly, specific $\alpha$ agonist effects. Experimental compounds contain partial antagonistic or more specific $\alpha, \gamma$ or even $\delta$ - agonistic effects.

PPAR- $\gamma$ is expressed ubiquitously in AT, heart, gut kidney, pancreas, spleen, muscle, macrophages and colon epithelium. Its stimulation plays a central role in TZD action; this is thought to improve insulin signaling in adipocytes, thus normalizing plasma FFA availability and, subsequently, glucose metabolism. The exact mechanism of the latter has, however, yet to be elucidated.

The role of adipocyte stimulation in the insulin sensitizing action of TZDs is unclear, but is considered important: PPAR agonists have distinct effects on lipid metabolism, including the stimulation of pre-adipocyte proliferation and differentation (240), (241) and the modulation 
of lipoprotein lipase (LPL) production and activity $(242,243)$. Mice completely lacking adipose tissue cannot be sensitized to insulin using TZDs (244).

There are also indications that TZDs increase expression of FA transporter proteins in adipose and other tissues; understanding these different FA kinetic influences might give insight into thus far poorly understood observations regarding FFA dynamics. For example, pure $\gamma-$ agonists lower plasma FFA concentrations without affecting TG concentrations, whereas $\alpha$ (co)agonists reduce both (245).

PPAR- $\alpha$ is most commonly found in the liver, but is also expressed in kidney, gut, skeletal muscle and AT. It is thought to mediate expression of genes promoting $\beta$ oxidation of fat (246), and although higher TZDs concentrations are needed for its stimulation than for PPAR$\gamma$, the achieved effects are stronger. Stimulation of PPAR- $\alpha$ has, indeed, been shown to affect mitochondrial action and is thought to (partly) mediate TZD effects on lipids (246). Moreover, PPAR- $\alpha$ agonism has been suggested to reduce the process of atherosclerosis by affecting cytokines, macrophages and foam cell formation, the inflammation process and reversed cholesterol transport. However, the pure- $\gamma$-agonist rosiglitazone has also been demonstrated to reduce the progress of intima-media thickness in carotid arteries of humans (247).

It is unknown to what extent PPAR- $\alpha$ and/or $-\gamma$ stimulation outside adipocytes is involved in the insulin-sensitizing effects of TZDs. Influencing intramuscular FA kinetics and fat transporter proteins might be particularly important herein. Effects of TZDs on FFA kinetics shall be discussed further in Chapter 4.5.3.

\subsubsection{Additional and adverse effects of thiazolidinediones}

TZDs have not been introduced without skepticism. Troglitazone, the prototype and first available TZD was retracted by the FDA in 1998 after causing a few cases of severe idiosyncratic liver failure. Several cases of liver damage have also been published for pio- and rosiglitazone (248-251); these however were reversible and not as a severe as with troglitazone. The drugs are now generally considered safe, even tough a follow-up of liver enzymes is still recommended after starting treatment.

Another important side effect of TZDs is weight gain. PPAR- $\gamma$ stimulation enhances the differentiation of pre-adipocytes to adipocytes and therewith increases total fat mass (Chapters 4.5.6). Sodium and water retention, also leading to hemoglobin lowering, adds to this.

However, this does not inhibit metabolic improvements. Besides insulin sensitization, reduction of the progression of atherosclerosis have been described, as well the lowering of lipids ((partial) $\alpha$-agonists only) and blood pressure (see also Chapter 4.5). Furthermore, beneficial effects on hepatic steatosis and lipoprotein particle sizes have been suggested (see Chapters 4.4.3 and 4.5.5). This might potentially broaden the indications for future TZD administration. 


\title{
4.4.3 Effect Of Pioglitazone On Biochemical Indices Of Non-Alcoholic Fatty Liver Disease In Upper Body Obesity
}

\author{
S Shadid and MD Jensen; Clinical Gastroenterology and Hepatology Sept \\ 2003; 1 (5): 384-7. \\ Endocrine Research Unit, Mayo Clinic, Rochester, Minnesota, USA
}

\begin{abstract}
Background: To report our observations on the change in liver function tests of volunteers receiving pioglitazone as part of a study of its effects on fatty acid metabolism. Treatment with other thiazolidinediones has been reported to ameliorate non-alcoholic fatty liver disease (NAFLD) in obese and diabetic humans, but whether pioglitazone has similar effects has not been reported.

Methods: Five of twenty upper body obese volunteers (10 men, 10 premenopausal women) had abnormal baseline liver enzymes ( 3 had ultrasound evidence of hepatic steatosis). All volunteers were treated with $30 \mathrm{mg}$ pioglitazone per day for $18 \pm 0.4$ weeks. Body composition, blood lipids and insulin sensitivity (intravenous glucose tolerance test) were assessed at baseline and after pioglitazone treatment.

Results: During pioglitazone treatment the liver enzyme abnormalities uniformly improved in subjects with evidence of NAFLD, primarily during the first 2 months. Some parameters of insulin sensitivity improved when measured after 18 weeks of pioglitazone treatment. Liver function tests remained normal in the 15 volunteers without evidence of NAFLD.

Conclusion: Liver function studies improved in obese volunteers with NAFLD during pioglitazone treatment. Although the nature of our observations does not prove a cause and effect relationship between pioglitazone treatment and improvement in liver enzymes, the time course and magnitude of improvement we observed may facilitate future research into thiazolidinedione treatment of NAFLD.
\end{abstract}




\section{Introduction}

Non-alcoholic fatty liver disease (NAFLD) is the most common liver disorder in the United States (252). Although generally benign, a portion of NAFLD cases may progress to nonalcoholic steatohepatitis (NASH) or even to cirrhosis $(252,253)$. Insulin resistance and obesity are major risk factors for developing NAFLD.

Neither its exact pathophysiology nor its association with the metabolic syndrome is fully understood; hepatic mitochondrial dysfunction (including impairment of fatty acid oxidation), oxidative stress and free radical formation have been proposed as pathogenic mechanisms $(254,255)$. Recently, a central, independent role has been attributed to increased plasma insulin concentrations, which are thought to induce hepatic fat accumulation $(252,253,255)$.

Improving insulin sensitivity may therefore be expected to improve NAFLD, if only by decreasing insulin concentrations. Substantial weight loss following bariatric surgery has been reported to decrease fatty infiltration in the liver, but also to worsen necro-inflammation and fibrosis (256). The latter is attributed to rapid mobilization of body fat and increased production of cytokines $(256,257)$. Because these phenomena would not be expected with pharmacological insulin sensitizers such as thiazolidinediones, these compounds might improve NAFLD without concomitant histological deterioration. Two preliminary reports have appeared describing the effects of troglitazone (now unavailable) (258) and rosiglitazone (259) on NAFLD in WHO class II obesity and type 2 diabetes respectively. In the course of studying the effects of pioglitazone on fatty acid metabolism in insulin resistant, non-diabetic upper body obese volunteers, we observed an apparent effect on the biochemical parameters of NAFLD. As best we can determine this is the first published report to demonstrate a potential therapeutic benefit of pioglitazone on non-diabetic, obese humans with NAFLD/NASH.

\section{Methods}

For this sub-study, the 20 volunteers randomized to the pioglitazone arm of the study described in Chapter 4.5.1 were analyzed separately. In summary, participants were given 30 $\mathrm{mg}$ of pioglitazone daily for 18 weeks and were monitored every 4 weeks for body weight, liver function tests and pill counts; liver enzymes were followed and analyzed throughout the treatment period. In 3 volunteers with liver enzyme abnormalities, ultrasound and hepatitis serologies were performed. None of the volunteers had risk factors for hepatic diseases or drank more than 2 glasses of alcohol per day.

Statistical analysis: values are expressed as mean \pm standard error of the mean unless otherwise stated. Statistical analyses were performed by t-tests, paired t-test and repeated measures-ANOVA. Parameters that were not normally distributed were $\log$ transformed prior to applying statistical methods in order to properly analyze the data. $\mathrm{P}$ values $<0.05$ were considered statistically significant.

\section{Results}

Of the 20 volunteers, 5 had elevated AST and/or ALT ( 4 men, 1 woman). Three of these 5 had been evaluated with hepatitis serologies and the structure of the liver was examined using 
ultrasound. The hepatitis serologies were negative and the ultrasound findings were compatible with NAFLD ${ }^{1}$.

During the course of pioglitazone treatment, ALT and AST improved and almost normalized (Figure 4.2) in all volunteers with liver enzyme abnormalities. The majority of the changes occurred during the first 2 months of pioglitazone use. There were no changes in liver enzymes in any of the 15 volunteers without biochemical evidence of NAFLD at baseline.

Table 4.4.1 Effect of pioglitazone on metabolic parameters of subjects with normal liver function tests (LFT) vs. Non-Alcoholic Fatty Liver Disease (NAFLD).

\begin{tabular}{|c|c|c|c|c|}
\hline & \multicolumn{2}{|c|}{ Normal LFTs } & \multicolumn{2}{|c|}{ NAFLD } \\
\hline & Before & After & Before & After \\
\hline Age (years) & $36 \pm 3$ & & $36 \pm 4$ & \\
\hline BMI $\left(\mathrm{kg} / \mathrm{m}^{2}\right)$ & $32.9 \pm 0.7$ & $33.4 \pm 0.8$ & $34.9 \pm 0.8$ & $36.0 \pm 1.4$ \\
\hline Total body fat (kg) & $37.9 \pm 1.9$ & $39.9 \pm 1.9 * * *$ & $38.9 \pm 3.1$ & $42.1 \pm 3.3^{* *}$ \\
\hline $\mathrm{Si}\left((\mathrm{mU} / \mathrm{l})^{-1} \bullet \mathrm{min}^{-1}\right)$ & $4.7 \pm 0.6$ & $7.4 \pm 1.3 *$ & $2.9 \pm 1.1$ & $4.3 \pm 1.3$ \\
\hline Fasting glucose (mg/dl) & $97 \pm 2$ & $94 \pm 2$ & $94 \pm 5$ & $93 \pm 4$ \\
\hline Fasting insulin $(\mu \mathrm{U} / \mathrm{l})$ & $11 \pm 1$ & $6 \pm 1 * *$ & $13 \pm 4$ & $8 \pm 1$ \\
\hline Fasting C-peptide (nmol/l) & $0.66 \pm 0.03$ & $0.50 \pm 0.03 * * *$ & $0.74 \pm 0.12$ & $0.52 \pm 0.09$ \\
\hline Triglycerides (mg/dl) & $153 \pm 12$ & $136 \pm 13$ & $228 \pm 49$ & $210 \pm 49$ \\
\hline HDL-cholesterol (mg/dl) & $36 \pm 2$ & $37 \pm 2$ & $28 \pm 4$ & $28 \pm 3$ \\
\hline Total cholesterol (mg/dl) & $197 \pm 8$ & $187 \pm 7$ & $191 \pm 12$ & $201 \pm 19$ \\
\hline
\end{tabular}

All parameters were measured in the overnight postabsorptive state. $* \mathrm{p}<0.05 * * \mathrm{p}<0.01 * * * \mathrm{p}<0.001$ before vs. after the intervention

Table 4.4.1 provides the changes in body composition and metabolic parameters in these volunteers. In participants with NAFLD there was a trend for improvement of $\mathrm{Si}(\mathrm{p}=0.17)$, fasting insulin $(\mathrm{p}=0.11)$ and $\mathrm{C}$-peptide $(\mathrm{p}=0.12)$. These parameters improved significantly, except fasting plasma glucose $(\mathrm{p}=0.09)$, in the 15 volunteers with normal liver enzymes. Serum lipid concentrations did not change significantly in either group.

\section{Discussion}

This appears to be the first report of a potential therapeutic benefit of pioglitazone in nondiabetic obese humans with evidence of NAFLD. As part of a study of fatty acid metabolism we treated upper body obese, insulin resistant volunteers with pioglitazone. We observed a significant improvement, but not complete normalization, in liver enzyme abnormalities. Liver enzymes were not adversely affected in those with normal initial values.

These results are consistent with reports of other thiazolidinediones. Troglitazone improved liver enzyme abnormalities in NAFLD ( 8 class II obese and one type 2 diabetic subject), with minimal amelioration of liver histology (258). In 9 type II diabetic subjects, rosiglitazone improved liver enzyme abnormalities and liver fat content measured by ${ }^{1} \mathrm{H}$ nuclear magnetic resonance.(259)

\footnotetext{
${ }^{1}$ Mild (1 woman) and mild to moderate (1 man) fatty liver infiltration. 1 US was performed in a different hospital
} 
Other reports describe coincidental improvements in abnormal liver enzyme abnormalities during troglitazone treatment of diabetic subjects $(260,261)$. Pioglitazone has been shown to improve insulin regulation of hepatic glucose production while reducing hepatic fat content in patients with type 2 diabetes (262). In addition, one retrospective (Acosta et al. Gastroenterology 2001;120 (Suppl): 2778) and two prospective (Sayal et al. Hepatology 2002; 36 (Suppl): 382A; Promrat et al. Gastroenterology 2003; 124 (Suppl): A708) studies of pioglitazone treatment of NAFLD/NASH have been reported in abstract form that are consistent with our findings. The eventual publication of these trials should provide important information on the

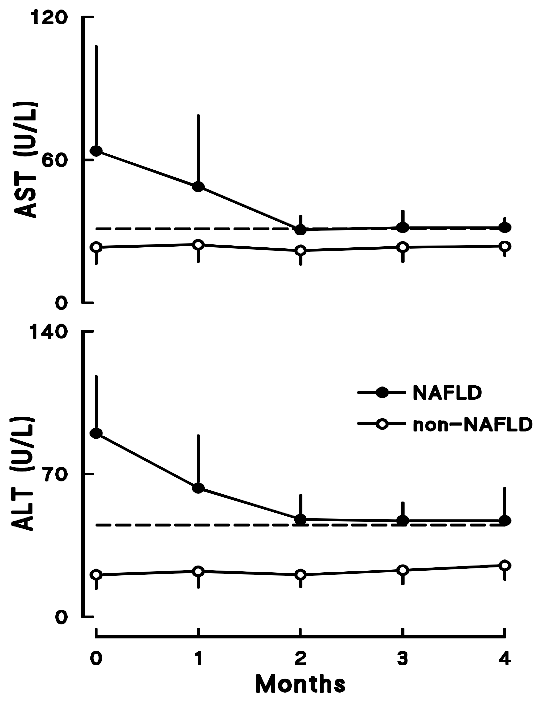

Figure 4.2 'Changes in AST and ALT over time during pioglitazone treatment'. Means and SDs are depicted The difference between the effect of pioglitazone on AST $(\mathrm{p}=0.05)$ and ALT $(p<0.0001)$ was significant between NAFLD and previously normal liver function tests. The dashed line represents the top of the normal range. histological changes accompanying pioglitazone treatment of NAFLD/NASH.

Although troglitazone was associated with hepatotoxic reactions in humans this has not been a major concern with pioglitazone and rosiglitazone. Only in transgenic mice were pioglitazone and rosiglitazone reported to aggravate NASH (263,264); this, however, does not represent the human physiologic situation since these mice lacked adipose tissue.

The relevance of our finding lies in the fact that class I obese volunteers are not normally treated with pioglitazone in the absence of overt diabetes. Our observations suggest that a prospective randomized clinical trial of pioglitazone to treat NAFLD may be reasonable. An agent that could prevent the progression of NAFLD to steatohepatitis or even cirrhosis would be useful. The weight gain and the rare idiosyncratic hepatic complications associated with thiazolidinedione use should not be a contraindication to researching this question.

The possible mechanism(s) of improvement in liver enzyme abnormalities with thiazolidinedione treatment cannot be defined from our study or the above-mentioned reports. The lack of change in serum lipids in our volunteers suggests that circulating lipids did not contribute to pioglitazone-associated improvement of liver enzyme abnormalities. Although some parameters of insulin sensitivity improved, others were unaffected despite consistently decreased insulin and C-peptide concentrations $(259,261)$. Results are therefore insufficient to distinguish between improving hyperinsulinemia and insulin sensitivity as the key variable.

Other possible mechanisms include modulation of cytokines (TNF- $\alpha$, leptin, adiponectin, resistin) or direct PPAR- $\alpha$ or $-\gamma$ receptor effects. The former has been demonstrated with thiazolidinedione administration in rodents and humans, and is likely mediated through gene transcription modulation (24,265-268). A direct involvement of PPAR-receptors has been suggested in the development of fatty liver disease; this might, conversely, be extended to the improvement in thiazolidinedione-mediated liver enzyme abnormalities, perhaps through altered insulin signaling within the hepatocyte.

Our results cannot prove a cause and effect relationship between pioglitazone treatment and improvement in liver function. The absence of a placebo control group, the small sample size 
and lack of histologic confirmation of NAFLD are limitations of this observation. Nevertheless, these findings are concordant with other publications and add to the impression that thiazolidinediones benefit obesity-associated liver disease.

In summary, these results are the first to suggest a beneficial effect of pioglitazone on NAFLD in non-diabetic, insulin resistant humans. Our observations provide dose and time course information for future studies of this phenomenon. Further research on the pathogenesis of NAFLD and the mechanism of pioglitazone effects on this disease may offer new therapies for a disease with potentially life-threatening complications. Differentiating between insulin resistance and hyperinsulinemia as the main pathophysiological variable in the development of NAFLD may further aid in treatment. 


\subsection{Pioglitazone Treatment Contrasted to Diet and Exercise}

\subsubsection{Aims of comparison}

The lack of insight into the pathophysiological and mechanistic aspects of TZDs and other treatment options of obesity-related insulin resistance prompted us to study the effects of PIO on different aspects of glucose and fatty acid metabolism in vivo, and to contrast these to Diet/ex-induced insulin sensitization.

We chose to study these effects in upper body obese, insulin resistant adults instead of in type 2 diabetic patients, since, in the latter, TZDs induce effects from a) insulin sensitization, b) direct TZD action and c) improved glycemic control on insulin action. Thus, choosing UBO adults eliminated the latter potentially confounding effect. The comparison with Diet/ex further allowed us to determine whether any observed effects were specific to pioglitazone, or could be reproduced non-specifically via another means of insulin sensitization.

39 upper body obese, insulin resistant, but non-diabetic humans were randomized to receive Diet/ex or PIO for 18-20 weeks. Before and after the respective interventions, various metabolic parameters were studied using a protocol, described in Chapter 4.5.2. This chapter also deals with the rationale and methods of our assays and calculations. Chapters 4.5.3- 4.5.6 describe various aspects of the metabolic changes observed before and after both intervention programs.

\subsubsection{Methods of Research}

\subsubsection{Subjects}

Written, informed consent was obtained from 68 healthy upper body obese, non-diabetic men and pre-menopausal women, 18-50 years of age and recruited via advertisements. 13 of these did not meet the inclusion criteria, 10 withdrew before actually entering the study, and 6 dropped out for various reasons (Figure 4.3), only one of which intervention (PIO)-related (angioneurotic edema (269)). The volunteers were weight stable for at least 6 months before entering the research program.

\section{Inclusion and exclusion criteria}

Inclusion criteria consisted of a body mass index (BMI) of $28-36 \mathrm{~kg} / \mathrm{m}^{2}$, combined with either a fasting plasma glucose between $100-126 \mathrm{mg} / \mathrm{dl}$, or one of the following three items: 1 . waist-to-hip ratio (WHR) $>0.85$ (women) or $>0.95$ (men), 2. CT measured visceral fat area $>$ $120 \mathrm{~cm}^{2}$ (women) or $>180 \mathrm{~cm}^{2}$ (men) or 3 . a ratio of visceral to total fat $>0.30$ (women) or $>$ 0.40 (men). 
Exclusion criteria were a history of coronary heart disease, atherosclerosis, known systemic illness, biochemical evidence of renal or liver failure, blood pressure $>160 / 90$, use of medication that could not be safely stopped 2 weeks prior to the study, smoking, pregnancy and breast-feeding.

Figure 4.3 Subjects

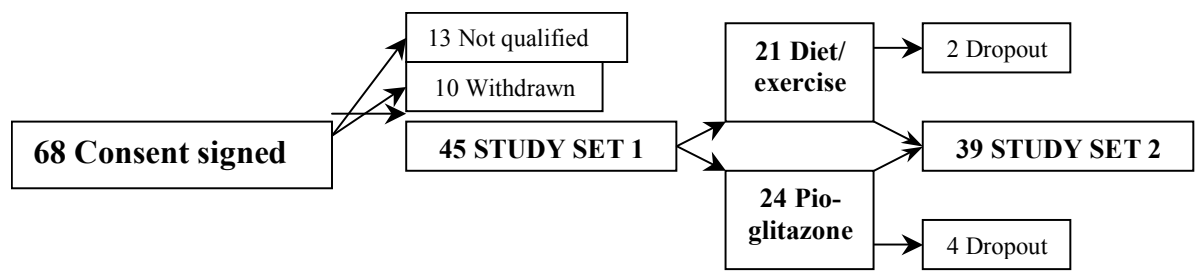

\section{General Outline}

The remaining 39 volunteers underwent blood testing (complete blood count, chemistry panel and lipid profile), an insulin-modified intravenous glucose tolerance test, CT measures of visceral fat area (60) and dual energy X-ray absorptiometry (DEXA, DPX-IQ, Lunar Radiation, Madison, WI)) for body composition assessment before and after the intervention.

Subsequently, the volunteers consumed an isocaloric diet for one week, consisting of $50 \%$ carbohydrates, $35 \%$ fat and $15 \%$ protein, prepared by the Mayo General Clinical Research Center (GCRC). The volunteers' energy requirements were estimated using the HarrisBenedict formula and usual daily activity. After the last evening meal the volunteer was kept fasted and admitted for the first study day.

Figure 4.4 (flow sheet) depicts the protocol followed during the admission. The study was originally meant to include intramuscular fatty acid metabolism in the analysis, reason why we included muscle biopsies and combined several radioactive with stable isotope fatty acid tracers (pulse-chase techniques) for the various sub-assessments intended. The results of the muscle studies are, however, not yet available.

After collection of baseline blood and breath samples, infusions of $\left[1-{ }^{14} \mathrm{C}\right]$ palmitate ${ }^{1}(0.3$ $\mu \mathrm{Ci} / \mathrm{min})$ and $\left[\mathrm{U}_{-}{ }^{13} \mathrm{C}\right]$ palmitate ${ }^{2}\left(6.0 \mathrm{nmol} \mathrm{kgFFM}^{-1} \bullet \mathrm{min}^{-1}\right.$; $)$ were started at $\mathrm{t}=-390 \mathrm{~min}$, followed by ${ }^{2} \mathrm{H}_{2}$ glucose ${ }^{2}\left(0.06 \mathrm{mg} \bullet \mathrm{kgFFM}^{-1} \bullet \mathrm{min}^{-1}\right)$ at $-330 \mathrm{~min}$. At $-60 \mathrm{~min},\left[9,10-{ }^{3} \mathrm{H}\right]$ oleate ${ }^{1}$ $(0.8 \mu \mathrm{Ci} / \mathrm{min})$ and $\left[\mathrm{U}_{-}{ }^{13} \mathrm{C}\right]$ oleate $^{2}\left(0.9 \mathrm{nmol} \bullet \mathrm{kgFFM}^{-1} \bullet \mathrm{min}^{-1}\right)$ were added.

Also at $\mathrm{t}=-60 \mathrm{~min}$, a hyperinsulinemic $\left(1.0 \mathrm{mU} \bullet \mathrm{kgFFM}^{-1} \bullet \mathrm{min}^{-1}\right)$, euglycemic clamp was started in half the volunteers. In those cases, FFA fluxes were expected to drop and, therefore, ${ }^{13} \mathrm{C}$ FFA enrichment to increase; to prevent the latter from rising above the top values of our standard curve (and thus lead to unreliable results) $\left[\mathrm{U}^{13} \mathrm{C}\right]$ palmitate and $\left[\mathrm{U}_{-}{ }^{13} \mathrm{C}\right]$ oleate infusion rates were reduced to $0.9 \mathrm{nmol} \bullet \mathrm{kgFFM}^{-1} \bullet \mathrm{min}^{-1}$.

The dose of insulin for this study was chosen to produce plasma levels in the midphysiological range, comparable to postprandial plasma insulin concentrations, which were high enough to produce observable effects, but low enough not to overwhelm detection of moderate changes in glucose and NEFA responses to insulin. The duration of the insulin clamp was chosen to ensure the achievement of a new steady state respiratory exchange ratio in order to accurately measure substrate oxidation.

\footnotetext{
${ }^{1}$ Perkin Elmer (NEN)

${ }^{2}$ Isotec, Matheson, USA
} 


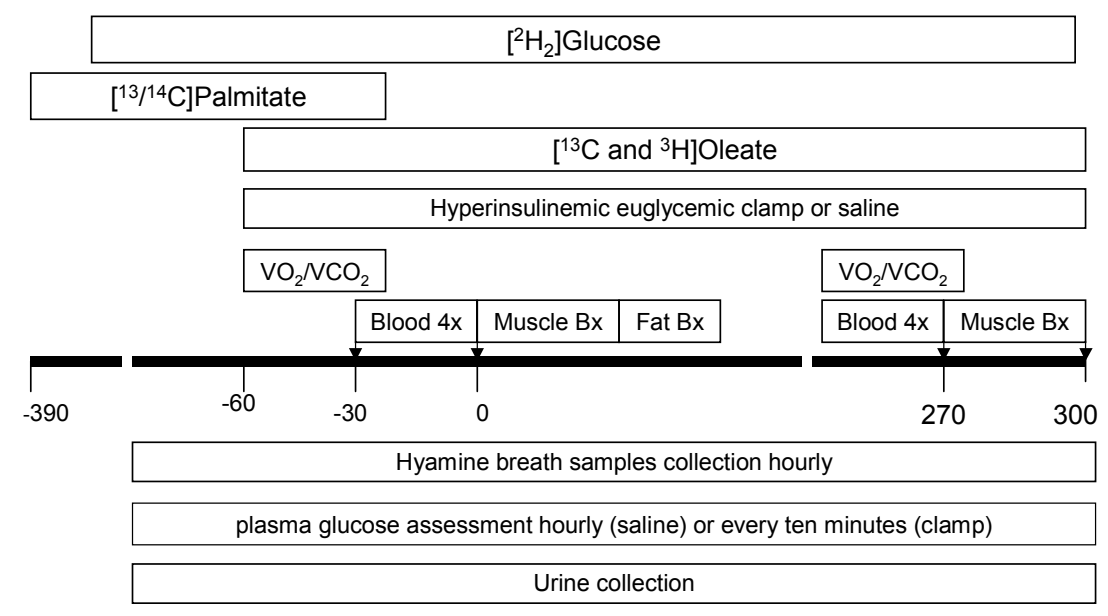

\section{Figure 4.4Flow Sheet}

At $\mathrm{t}=0$ minutes, a biopsy was taken from the vastus lateralis muscle, after which the palmitate tracer infusions were stopped. Subsequently, subcutaneous fat was aspirated from femoral and abdominal depots under local anesthesia using sterile technique. A $5 \mathrm{lb}$ weight was placed around the contralateral ankle of the volunteer, who was asked to lift this leg 10 times every 10 minutes to increase quadriceps energy expenditure. This was done to mimic light "daily" leg activity (as opposed to complete bed rest). Breath samples were collected every hour from -120 min onward for measurement of ${ }^{14} \mathrm{CO}_{2}$ specific activity (SA). Blood samples were drawn at 10 minute intervals between $\mathrm{t}=30$ and $\mathrm{t}=0$ minutes, and again between $\mathrm{t}=270$ and $\mathrm{t}=300 \mathrm{~min}$.

Urine was collected from $\mathrm{t}=-180$ min onward. Breath samples were taken at baseline and hourly from $t=-120$ min onward. Indirect calorimetry was performed at $t=-60$ and $t=240$ min respectively.

At $\mathrm{t}=300 \mathrm{~min}$, a second muscle biopsy was taken from the contralateral thigh, after which the volunteer was given a meal and dismissed from the GCRC.

Volunteers were then randomized to receive $30 \mathrm{mg}$ of pioglitazone (PIO) daily or a combination of a hypocaloric diet ( $-500 \mathrm{Kcal}$; same nutrient composition as above), an aerobic exercise program and a biweekly behavior modification program (Appendix 4) for 18-20 weeks. The PIO group was seen once every 4 weeks for weight measurement, liver function tests and pill counts.

After completion of the intervention program all baseline measurements and tracer/biopsy studies were repeated, following the exact same protocol.

\section{Diet And Exercise Protocol}

The diet (500 Kcal deficit) consisted of $50 \%$ carbohydrates, $35 \%$ fat and $15 \%$ protein. The subject was monitored and advised by the dietician once every 4 weeks, and instructed to a program of aerobic exercise of his or her choice, starting at 3 times 15-20 minutes weekly at 
$50 \%$ of their heart rate reserve. They were instructed to increase this gradually to 4 times weekly for 50 minutes at $60-70 \%$ of heart rate reserve (Appendix 3). This would approximate a $1500 \mathrm{kCal}$ of maximal energy expenditure per week. An exercise physiologist gave individualized exercise instructions at least once during the program. Exercise logs were viewed weekly to monitor compliance.

In addition, behavior modification sessions in groups of 3 or fewer were given by a physician once every 2-3 weeks, or more if needed. This was based on a modified LEARN program (230).

\subsubsection{Assays And Methods}

\section{Hormones and cytokines}

Adipokines, plasma: Adiponectin: Human Adiponectin double antibody radio-immunoassay kit (Linco Research, Inc. St. Louis, MO 63304); High Sensitivity C-Reactive Protein: Hitachi 912 chemistry analyzer by a latex particle enhanced immunoturbidimetric assay (Kamiya Biomedical Corp., Seattle, WA 98188); High Sensitivity Interleukin-6: quantitative two-site enzyme immunoassay from R \& D Systems, Minneapolis, MN 55413. Resistin: quantitative two-site enzyme immunoassay (Linco Research). High Sensitivity TNF- $\alpha$ : quantitative twosite enzyme immunoassay ( $\mathrm{R} \& \mathrm{D}$ Systems).

C-peptide: direct, double antibody sequential radioimmunoassay (Linco Research); Catecholamines in plasma: reversed phase HPLC with electrochemical detection after extraction with activated alumina. Growth Hormone and insulin: chemiluminiscence method with Access Ultrasensitive Immunoenzymatic Assay system; Beckman, Chaska, MN

\section{Triglycerides and Fatty Acids}

FFA: HPLC (high performance liquid chromatography); this is described in Chapter 4.5.2. Stationary phase: $25 \mathrm{~cm}$ APEX ODS silica column (Jones chromatography Ltd, Mid Glamorgan, UK). Particle size: 5 micron. Inside diameter: $4.6 \mathrm{~mm}$. Pressure: 62 bar. Mobile phase: $78 \%$ acetonitrile, $5 \%$ methanol, $17 \% \mathrm{H} 2 \mathrm{O}$. Internal standard: $\left[{ }^{2} \mathrm{H}_{31}\right]$ palmitate. Triglycerides : Hitachi 912 chemistry analyzer using Technicon triglyceride reagent (Bayer Corp, Tarrytown NY 10591).

Glucose: the Hitachi 912 Chemistry Analyzer using the hexokinase reagent from Boehringer Mannheim (Indianapolis, IN 46256), or the Beckman glucose analyzer (Beckman Instruments, Fullerton, CA).

Glucose enrichment: gas chromatography/mass spectrometry (73).

Intravenous glucose tolerance test (IVGTT)

An intravenous injection of $0.33 \mathrm{~g} / \mathrm{kg}$ dextrose $50 \%$ at $\mathrm{t}=0$ was followed by a $0.02 \mathrm{U} / \mathrm{kg}$ insulin bolus at $\mathrm{t}=20 \mathrm{~min}$. Glucose (Beckman glucose analyzer) and insulin measurements were performed at $\mathrm{t}=0,2,4,8,10,18,24,32,40,6070,120$ and 180 minutes. Data were analyzed using Bergman's minimal model $(270,271)$. 
Determination of Fat Compartments with CT and DEXA

Total fat mass was assessed by routine measurement using DEXA scanning. Compartmental fat volumes were assessed by manual outlining of head, thorax and upper and lower extremities (Figure 4.5).

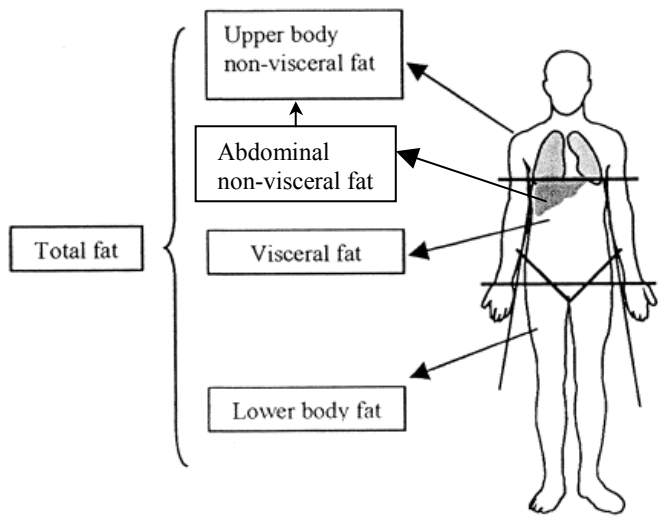

Figure 4.5 Determination of abdominal fat using DEXA scanning and computed tomography

A horizontal line is drawn (height of symphysis pubis) to exclude the lower extremities from the calculations, and a second one at the height of the diaphragm. The latter and two additional diagonal lines separate the upper body and extremities from the abdomen. Thus an estimate can be made of total abdominal fat. Total fat equals the sum of all subcompartments.

Subtraction of these from total fat yielded abdominal fat, which was subdivided into visceral fat (VISC) and abdominal subcutaneous (SC) fat using the visceral to total fat ratio obtained with a single slice $\mathrm{CT}$ image at the $\mathrm{L}_{2-3}$ interspace.

Euglycemic, hyperinsulinemic clamp

Following an insulin bolus $(0.7 \mathrm{mU} / \mathrm{kgFFM})$, insulin was infused at a rate of $1.0 \mathrm{mU} \bullet \mathrm{kgFFM}^{-1}$ - $\min ^{-1} .{ }^{2} \mathrm{H}_{2}$-labeled $50 \%$ dextrose was infused at a variable rate to keep plasma glucose levels between 90 and $95 \mathrm{mg} / \mathrm{dl}$. Plasma glucose was measured using a Beckman Glucose Analyzer (see above) every 10 minutes throughout the duration of the clamp. The participants receiving the saline infusion in place of the insulin clamp were infused with $0.06 \mathrm{mg} \bullet \mathrm{kg}^{-1} \bullet \mathrm{min}^{-1} \mathrm{of}^{2} \mathrm{H}_{2}$ glucose.

Determination of specific activity $(S A)$ of ${ }^{14} C$ and ${ }^{3} H$ lipids

Specific activity (SA) was counted after lipid extraction (adipose tissue) or from HPLC fractions (plasma) in a beta-counter (Wallac 1409 liquid scintillation counter) after addition of water and standard scintillation cocktail (Opti-fluor ${ }^{\mathrm{TM}}$, Packard Bio Science, Groningen, The Netherlands).

\section{Lipid extraction from adipose tissue}

AT was washed with saline through Nitex Nylon Fiber 250/50. A portion was directly frozen in liquid nitrogen and stored at $-80 \mathrm{C}$ for future assays.

The remaining tissue was added to $15 \mathrm{ml}\left[\mathrm{CHCl}_{3}\right.$ : methanol (2:1)] and incubated in a cold room for at least 2 days. $3.75 \mathrm{ml} \mathrm{88 \%} \mathrm{KCL}$ was added, and after centrifuging the lipid layer could be removed (with pipette and filter), dried down and counted for specific activity (SA). 
Fat cell sizing

Adipose tissue from biopsies was rinsed with saline and digested in a HEPES/collagenase solution $\left(37^{\circ} \mathrm{C}\right)$ (Sigma Type II C-6885). Adipocytes were isolated by centrifugation and the cells were stained with methylene blue to allow identification of nuclei. At 10x magnification a digital photograph was taken, after which $>100$ cells were sized and averaged manually. The latter was validated against direct microscopic counting, i.e. without the intervention of a photograph. Histograms were graphically and numerically displayed.

\section{Treadmill testing and $\mathrm{VO}_{2} \max$}

Maximal oxygen consumption $\left(\mathrm{VO}_{2} \mathrm{max}\right)$ was determined by a graded exercise test performed on a Quinton (Seattle, WA) motor driven treadmill using a modified Bruce protocol (72). Oxygen consumption was measured using an open circuit gas exchange system (MedGraphics, St. Paul, MN). Heart rate and rhythms were monitored continuously via a 10-lead ECG and rating of perceived
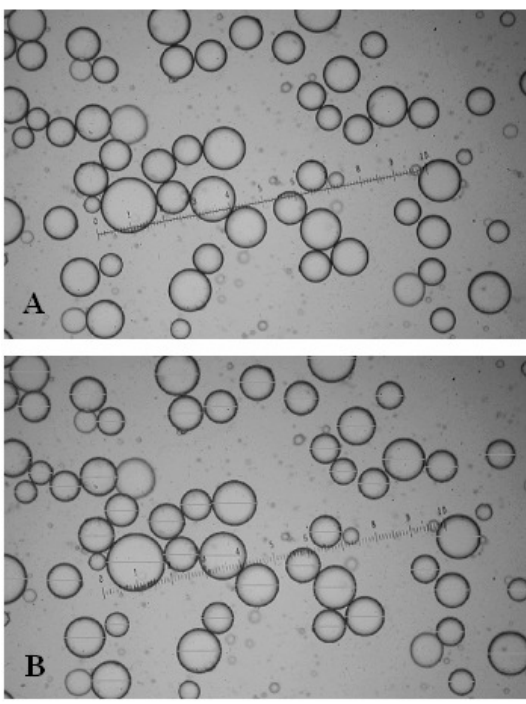

Figure 4.6 Fat cells prepared for microscopic sizing $(A)$ and computer sizing (B) exertion (RPE) was recorded during the last 20 seconds of each two-minute stage. Termination of the test was based hereupon.

The difference between resting and maximal heart rate defined the heart rate reserve, which was used to determine exercise intensity goals for those in the diet/exercise program.

Ventilatory (lactate) threshold was defined as the first $\mathrm{VO}_{2}$, exceeded by $\mathrm{VCO}_{2}$, and expressed both as an absolute number and relatively to $\mathrm{VO}_{2} \max$. Higher results indicate better physical fitness.

\section{Urinary nitrogen analysis}

In a GM7 analyzer (Analox Instruments Ltd, London, UK), urine samples were mixed with a GMRD-086 kit. Lyophilised urease/GDH and NADH reagents were divided equally for part reconstitution. Peroxidase (POD) enzyme was dissolved in tris-succinate buffer and placed in the analyzer reagent reservoir.

\subsubsection{Principles and Calculations of Assays}

\section{Hyamine breath samples}

Breath samples were caught in a highly acidophilic $1 \mathrm{ml} 0.5 \mathrm{mM}$ hyamine (Benzethonium Hydroxide $1.0 \mathrm{M}$ in Methanol) solution. Its $1: 1$ binding with ${ }^{\left(14 \text { or }{ }^{12}\right)} \mathrm{CO}_{2}$, neutralizing the $\mathrm{pH}$, is indicated by a color tracer (thymolphthalein).

The SA $(\mathrm{dpm} / 0.5 \mathrm{mM})$ of ${ }^{14} \mathrm{C}$ was assessed in a scintillation counter (see above) and doubled to obtain the amount of disintegrations per minute (dpms) per $\mathrm{mM} \mathrm{CO}_{2}$. This was converted to $\mathrm{dpm} / \mathrm{ml}$ (divided by $22.4 \mathrm{ml} / \mathrm{mM}$ ).

$\mathrm{CO}_{2}$ production $(\mathrm{ml} / \mathrm{min})$, measured simultaneously by indirect calorimetry, allowed for conversion into $\mathrm{dpms} / \mathrm{min}$. 
FFA flux and clearance: basic principles and calculations

\section{A. Basic principles of FFA flux}

FFA flux represents the amount of FFA, released from AT and taken up by peripheral tissues per time unit. At steady state, release rates by definition equal uptake rates; if we assume that the vast majority of plasma FFA originate from AT only, adipose tissue lipolysis rates equal FFA release rates, which equal FFA flux. In our studies, we used continuous infusion of labeled tracer to assess FFA flux, assuming that labeled FA are handled identically to natural FA and that FFA reach peripheral tissues via blood only. Palmitate and oleate represent all FFA in our studies, since these comprise $20-28$ and $35-40 \%$ of plasma FFA respectively (272).

Infusion of ${ }^{14} \mathrm{C}$-palmitate at a constant rate into the circulation will create a ${ }^{14} \mathrm{C}$ specific activity (SA) of plasma palmitate. At steady state, this ${ }^{14} \mathrm{C}$-palmitate SA can be used to calculate the rates at which unlabeled palmitate enters and exits the circulation (both rates, again, being equal at steady state). A lower SA indicates more unlabeled FFA entering the circulation whereas a higher SA indicates less FFA release (figure 4.7).

Figure 4.7 Free Fatty Acid Flux FFA $\bigcirc$ are released into the circulation by adipose tissue and taken up by peripheral tissues. The rate at which this occurs is denoted as FFA flux rate. At steady state, release equals clearance. Constant infusion of radioactively labeled FFA can therefore be used as a marker FFA flux rate, since infusion rates are known and clearance of labeled and unlabeled FFA are equal.

The specific activity of total FFA measured (dpm/nmol FFA) indicates the relative presence of labeled FA (enrichment). High lipolysis rates (i.e high FFA flux) will cause more dilution of the infused labeled FFA and therefore lead to a low specific activity (middle figure) and vice versa.

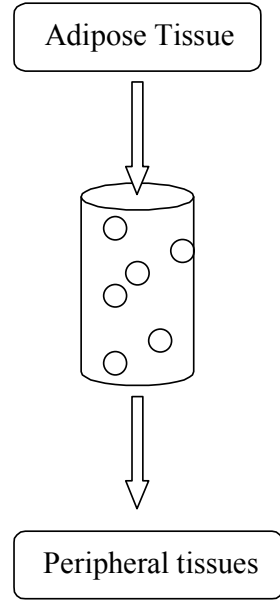

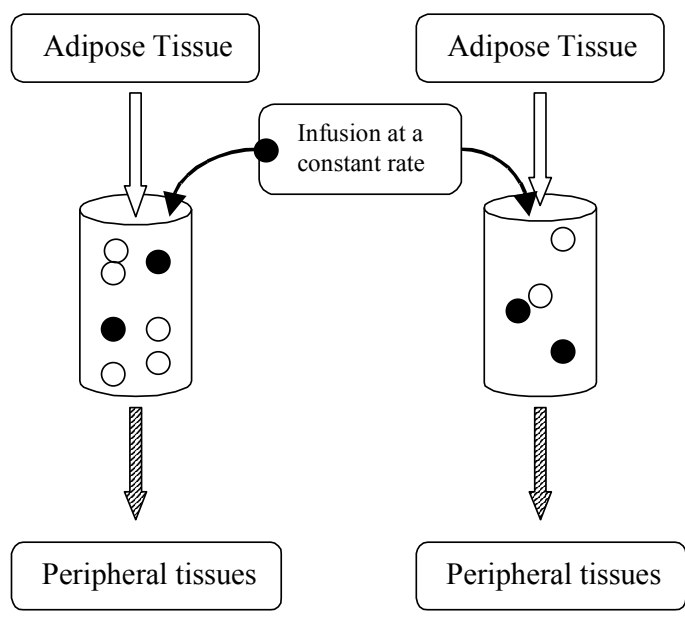

HIGH FLUX

\section{LOW FLUX}

$B$.

Calculation of FFA flux

Plasma samples from 4 time points (see flow sheet) were analyzed for total palmitate and oleate concentrations and SA for ${ }^{14} \mathrm{C}$ and ${ }^{3} \mathrm{H}(\mathrm{dpm} / \mathrm{nmol})$ and the results averaged to estimate average flux over the 30 min interval. 
The content of radiolabeled palmitate and oleate of the infusates was measured $(\mathrm{dpm} / \mu \mathrm{l})$, which allowed us to know the tracer infusion rate in dpm per minute.

FFA flux $(\mu \mathrm{mol} / \mathrm{min})$ was calculated as the SA of the infusates $(\mathrm{dpm} / \mathrm{min})$ divided by the SA of plasma oleate or palmitate $(\mathrm{dpm} / \mu \mathrm{mol} /)$. Similar approaches can be used with fatty acid tracers labeled with stable isotopes, using enrichment instead of SA.

\section{FFA clearance}

FFA clearance represents the efflux of FA from blood into tissues relative to plasma concentrations. It is calculated by dividing FFA flux by FFA concentration. Both FFA flux and clearance can be influenced by insulin.

\section{Glucose turnover}

Glucose turnover rates were assessed using the same principles and formulas as for FFA flux adapted for stable isotopes. Using gas chromatography/mass spectroscopy, ${ }^{2} \mathrm{H}_{2}$ enrichment of glucose could be assessed and expressed in mole percent enrichment (MPE). Glucose disposal rate $(\mathrm{mg} / \mathrm{kgFFM} / \mathrm{min})$ then equaled (glucose infusion rate/ ${ }^{2} \mathrm{H}_{2}$ enrichment)- ${ }^{2} \mathrm{H}_{2}$ glucose infusion rate. The latter subtraction of glucose infusion rate was done to correct for the glucose mass infused.

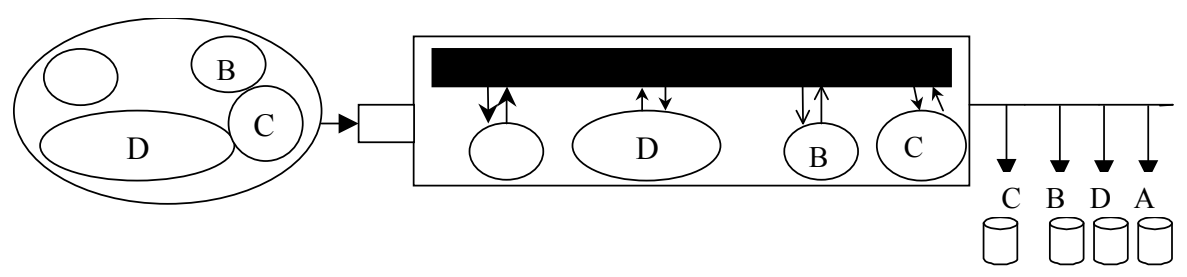

Figure 4.8 Principles of High Performance Liquid Chromatographv (HPLC)

\section{High Performance Liquid Chromatography (HPLC)}

High Performance Liquid Chromatography (HPLC) is a technique based on the chromatographic separation of the components of a solution (mobile phase), by forcing it over a column (stationary phase). When the solution and its components interact with the stationary phase, i.e. the column, an interaction is created between the solvents and the stationary phase. The unique chemical qualities of the solvents and compounds will lead to different retention within the column. Therefore, each compound passes through the column at a unique rate.

The substances can be collected after leaving the column and can be further used if needed. (see figure 4.8).

\section{Urinary Nitrogen Excretion}

Urine was collected during approximately 9 hours. Samples were analyzed for urea content $(\mathrm{mmol} / \mathrm{L})$, extrapolated to $24 \mathrm{hr}$ excretion and converted to $\mathrm{g}$ of urea, and subsequently to grams of Nitrogen excretion per 24 hours. 


\section{Indirect calorimetry and substrate oxidation}

\section{A. Indirect calorimetry}

Indirect calorimetry was performed using a Delta-Trac Metabolic Cart (SensorMedics, Yorba Linda, CA) that was calibrated every morning before use with reference gases and every six months with an alcohol burn test. Volunteers lay still, but awake in bed and were asked not to move or talk. A clear, plastic hood, placed over their heads measured $\mathrm{O}_{2}$ consumption and $\mathrm{CO}_{2}$ production.

The results of the first 4 out of 20 minutes were not used for calculations to allow for stabilization of breathing.

\section{B. Substrate oxidation}

Assessment of substrate oxidation from gaseous exchange was based on previous descriptions (273). In short, it is based on the fact that carbohydrates $(\mathrm{CH})$, fat and protein are the only oxidative substrates, all of which are converted into $\mathrm{CO}_{2}$ and $\mathrm{H}_{2} \mathrm{O}$ (and nitrogen in the case of proteins).

Oxidation of $1 \mathrm{mmol}$ glucose uses $6 \mathrm{mmol}$ of oxygen and produces $6 \mathrm{mmol}$ of $\mathrm{CO}_{2}$ :

$\mathrm{C}_{6} \mathrm{H}_{12} \mathrm{O}_{6}+6 \mathrm{O}_{2} \longrightarrow 6 \mathrm{CO}_{2}+6 \mathrm{H}_{2} \mathrm{O}$

By definition, the Respitatory Quotient (RQ), equaling $\mathrm{VCO}_{2} / \mathrm{VO}_{2}$, in the latter case is 1 .

For fat, typically palmitoyl-stearoyl-oleoyl-glycerol $\left(\mathrm{C}_{55} \mathrm{H}_{104} \mathrm{O}_{6}\right)$ is used (273). Its oxidation uses $78 \mathrm{mmol}$ of oxygen and produces $55 \mathrm{mmol} \mathrm{CO}_{2}$ per mmol, yielding a substantially lower $\mathrm{CO}_{2} / \mathrm{O}_{2}$ ratio and hence a lower RQ.

Thus, a higher RQ indicates a higher $\mathrm{CH}$ oxidation relative to fat and vice versa. The contribution of proteins is accounted for by urinary nitrogen production $(\mathrm{N}, \mathrm{g} / \mathrm{min})$, so that fat and $\mathrm{CH}$ oxidation can be described mathematically.

Fat oxidation $(\mathrm{g} / \mathrm{min})=1.67 \mathrm{VO}_{2}(1 / \mathrm{min})-1.67 \mathrm{VCO}_{2}(1 / \mathrm{min})-1.92 \mathrm{~N}$

Carbohydrate oxidation $(\mathrm{g} / \mathrm{min})=4.55 \mathrm{VCO}_{2}-3.21 \mathrm{VO}_{2}-2.87 \mathrm{~N}$

\subsubsection{Statistical Analyses}

Statistical analyses were done using SPSS, SAS and JMP programs. The specific statistical methods used are described in the chapters applicable.

Values are expressed as mean \pm standard error of the mean; $\mathrm{P}$ values $<0.05$ were considered statistically significant. 


\title{
4.5.3 Pioglitazone increases FFA Clearance
}

\author{
Samyah Shadid and Michael D. Jensen ' 'Pioglitazone Increases Non- \\ Esterified Fatty Acid Clearance in Upper Body Obesity'; Diabetologia 2006 \\ Jan;49(1):149-57
}

Endocrine Research Unit, Mayo Clinic, Rochester, Minnesota, USA

${ }^{1}$ Current address: VieCuri Medical Center, Venlo, The Netherlands

\begin{abstract}
Aims/Hypothesis: Plasma non-esterified fatty acid (NEFA) concentrations are largely determined by adipose tissue lipolysis. Insulin suppression of lipolysis is commonly impaired with insulin resistance and improves with thiazolidinedione treatment of type 2 diabetes. These studies were designed to assess thiazolidinedione effects on NEFA (oleate) metabolism independent of improved glycaemic control.

Methods: We measured plasma oleate concentration and flux $\left(\left[{ }^{3} \mathrm{H}\right]\right.$ oleate $)$, glucose kinetics $\left(\left[6-{ }^{2} \mathrm{H}_{2}\right]\right.$ glucose) and substrate oxidation (indirect calorimetry) before and after pioglitazone (30 mg/day for $\sim 20$ weeks) in 20 non-diabetic, upper body obese adults. To assess the effects of improved insulin sensitivity per se we performed the same measures in a matched group of volunteers treated with diet/exercise. Half of the volunteers underwent these measures during a hyperinsulinemic, euglycaemic clamp and half during a (control) saline infusion before and after the intervention.

Results: Both interventions increased insulin-stimulated glucose disposal and reduced plasma oleate concentrations during the insulin clamp. After diet/exercise, oleate flux decreased $(\mathrm{p}=0.03)$ during the insulin clamp and oleate clearance remained unchanged $(\mathrm{p}=0.55)$, whereas in the pioglitazone group oleate flux during the clamp was unchanged $(p=0.97)$, oleate clearance increased $(\mathrm{p}=0.003)$. Oleate clearance in the saline control condition was increased in the pioglitazone group compared with the diet/exercise group $(\mathrm{P}=0.02)$.

Conclusion: In insulin resistant, non-diabetic adults, pioglitazone increases NEFA clearance during physiologic hyperinsulinemia, whereas improved insulin sensitivity achieved by diet/exercise does not alter NEFA clearance but enhances insulin suppression of NEFA release. This action of pioglitazone may contribute to improved glucose metabolism in type 2 diabetes.
\end{abstract}




\section{Introduction}

Insulin resistance is commonly present in upper body obesity $(9,37,274)$ and type 2 diabetes $(45,69)$. This is manifested by impaired glucose disposal and higher plasma non-esterified fatty acid (NEFA) concentrations. Plasma NEFA concentrations are largely determined by effective adipose tissue lipolysis, as assessed by measurements of NEFA release (75), but are also regulated by NEFA clearance $(66,275)$. Lipolysis is abnormally increased under both basal $(37)$ and insulin suppressed conditions $(37,38)$ in upper body obesity and type 2 diabetes $(45,69)$. The resultant higher NEFA concentrations $(66)$ are thought to contribute to insulin resistance at the level of muscle $(110,276)$ and liver (277), hypertriglyceridemia (278) and abnormal vascular function (279). The ability of insulin to suppress plasma NEFA concentrations following meal ingestion is thought to play an important role in determining peripheral glucose disposal (110) and suppression of hepatic glucose release (277). Thus, the effects of diabetes treatment agents on postabsorptive and insulin suppressed NEFA are of equal interest.

Interventions that reduce insulin resistance also improve the metabolic abnormalities associated with upper body obesity (280) and enhance glucoregulation in type 2 diabetes. Examples include diet/exercise and TZDs (281). Thiazolidinediones, PPAR $\gamma$ agonists, have been reported to improve the insulin suppression of lipolysis in type 2 diabetes $(259,282-284)$; this could account for the improved insulin regulation of glucose disposal because of reduced NEFA concentrations.

Imputing basic physiological actions of TZDs from their effects in type 2 diabetes is confounded by effects of improved glycaemic control on insulin action, however. Lowering plasma glucose concentrations in type 2 diabetes improves insulin action with respect to glucose metabolism even when insulin is used as the treatment (285). Although similar studies do not appear to have been conducted with respect to NEFA metabolism in type 2 diabetes, there are reports that in vitro lipolysis of human fat cells is affected by variations in the extracellular glucose concentrations $(286,287)$. To avoid the potential confounding variable of disparate improvements in diabetic control with different treatments we examined the physiological effects of pioglitazone on fatty acid metabolism in non-diabetic, upper body obese men and women in whom insulin regulation of NEFA metabolism is known to be abnormal $(37,38)$. To determine whether any observed effects were specific to pioglitazone, or could be reproduced non-specifically via another means of insulin sensitization, we also studied a comparable number of volunteers before and after a diet/exercise induced weight loss.

\section{Methods}

\section{Subjects and Study Protocol}

Available data from all subjects described in Chapter 4.5.2 were analyzed, including the ones who did not finish both studies. For this analysis, we used the afternoon data from the $[9,10$ $\left.{ }^{3} \mathrm{H}\right]$ oleate $(0.8 \mu \mathrm{Ci} / \mathrm{min})$ infusion, to allow for steady state conditions to occur in the clamp studies. The flow sheet is depicted in Figure 4.5.

For easier reading, the study protocol is described again below.

After baseline testing (body composition, complete blood count, chemistry panel, lipid profile, intravenous glucose tolerance test (288)) and the one week isocaloric diet (Chapter 4.5.2), volunteers were admitted to the Mayo GCRC where a blood sample was collected for background plasma oleate specific activity (SA) and glucose enrichment. At $0600 \mathrm{hrs}$ the next morning an infusion of $\left[6{ }^{2} \mathrm{H}_{2}\right]$ glucose $\left(0.06 \mathrm{mg} \mathrm{kgFFM}^{-1} \bullet \mathrm{min}^{-1}\right)$ was begun to trace glucose 
kinetics, followed by a $\left[9,10-{ }^{3} \mathrm{H}\right]$ oleate $(0.8 \mu \mathrm{Ci} / \mathrm{min})$ infusion at $0800 \mathrm{hrs}$. In 19 volunteers, a hyperinsulinemic $\left(1.0 \mathrm{mU} \bullet \mathrm{kgFFM}^{-1} \bullet \mathrm{min}^{-1}\right)$, euglycaemic clamp was started at $0800 \mathrm{hrs}$ using $\left[6-{ }^{2} \mathrm{H}_{2}\right]$ glucose-labeled $50 \%$ dextrose; the other 20 participants received a saline infusion. The dose of insulin for this study was chosen as one in the mid-physiological range (comparable to plasma insulin concentrations obtained following meal ingestion) and to be sure that moderate changes in glucose and NEFA responses to insulin could be detected if they occurred.

The duration of the insulin clamp was chosen to be sure that a new steady state respiratory exchange ratio would be achieved in the insulin clamp group in order to accurately measure substrate oxidation. Blood samples were obtained at 10 min intervals between 1330$1400 \mathrm{hrs}$ for the measurement of plasma hormone and catecholamine concentrations, as well as plasma oleate, and glucose flux rates. Indirect calorimetry was performed at $1330 \mathrm{hrs}$ with a DeltaTrac Metabolic Cart (Yorba Linda, CA, USA). Urine was collected for measurement of nitrogen excretion throughout the study.

The volunteers were then randomized to either a diet/exercise program or pioglitazone $30 \mathrm{mg}$ daily for 18-20 weeks (288), after which all studies were repeated, identically to the preintervention studies. The weight loss program consisted of a diet designed to induce a 500 $\mathrm{Kcal} /$ day deficit ( $55 \%$ carbohydrates, $30 \%$ fat, $15 \%$ protein), an aerobic exercise program and biweekly behavior modification instructions (modified 'LEARN' program (230)). The pioglitazone treated volunteers continued their previous life style (diet and physical activity) and were monitored every 4 weeks for body weight, liver function tests and pill counts. The second study for each volunteer was identical to their first study, including the weight maintaining diet, documented by daily GCRC weights, for one week prior to their second admission.

\section{Analysis of plasma samples}

Only plasma triglyceride analysis is not described in Chapter 4.5.2. TG concentrations were measured using a centrifugal auto-analyzer (58) and plasma triglyceride $S A$ was measured by extracting the lipids from plasma using standard techniques (289), and then specifically isolating and measuring the radioactivity in the triglyceride using an HPLC-based approach (42). We corrected for incomplete recovery by measuring the glycerol concentration in a hydrolyzed aliquot of the triglyceride HPLC fraction. This method allowed us to measure the specific concentration of ${ }^{3} \mathrm{H}$ labeled triglyceride in plasma $(\mathrm{dpm} / \mathrm{ml})$ irrespective of the presence of other ${ }^{3} \mathrm{H}$ labeled compounds $\left({ }^{3} \mathrm{H}_{2} \mathrm{O},{ }^{3} \mathrm{H}\right.$-oleate, etc $)$.

\section{Calculations}

NEFA flux is the amount of FA released into the circulation via adipose tissue lipolysis and taken up by tissues per time unit. At steady state, the rate of NEFA release equals NEFA uptake. In these studies we specifically measured oleate, the major NEFA in plasma, as the indicator of NEFA flux.

Steady state plasma oleate (and NEFA) concentrations and specific activity were achieved in each study. Steady state rates of appearance $(\mathrm{Ra})$ and disappearance $(\mathrm{Rd})$ of oleate and glucose were calculated using the mean SA or enrichment values and tracer infusion rates $(37,73)$. Endogenous glucose production during the insulin clamp was calculated by subtracting the exogenous glucose infusion rate from total glucose appearance. Oleate clearance (liters $/ \mathrm{min}$ ) was calculated by dividing oleate $\mathrm{Rd}(\mu \mathrm{mol} / \mathrm{min})$ by oleate concentration $(\mu \mathrm{mol} / \mathrm{L})$. Because the groups were well matched for resting energy expenditure (REE) and FFM the oleate flux data are presented as $\mu \mathrm{mol} / \mathrm{min}$ rather than dividing by units of weight or body composition (66). Matching for relevant variables is preferable to using numerator/denominator approaches to present NEFA flux data because it prevents drawing spurious conclusions (77); NEFA flux is most strongly predicted by REE (66). Substrate oxidation was 
assessed using $\mathrm{CO}_{2}$ production relative to $\mathrm{O}_{2}$ consumption rates and urinary nitrogen excretion (273).

\section{Statistical analysis}

Values are expressed as mean \pm standard error of the mean. Statistical comparisons between men and women in the diet/exercise and pioglitazone groups before and after interventions were performed using a $2 \times 2 \times 2 \times 2$ repeated measures ANOVA with factors for sex (men vs. women), group (diet/exercise vs. pioglitazone) study (saline vs. insulin clamp) and time (the repeated measure - before and after intervention). If statistically significant differences were found, follow up non-paired or paired t-tests were performed. If the direction of the difference was predicted from the literature (for example, insulin stimulated glucose disposal increasing with diet/exercise or pioglitazone) a one-sided t-test was used instead of a two-side comparison. $\mathrm{P}$ values $<0.05$ were considered statistically significant. We applied a Bonferroni correction when multiple comparisons were made that were not part of the initial plan for statistical analysis.

\section{Results}

\section{Subject Characteristics}

As we previously reported (288) the baseline, anthropometric, biochemical, and metabolic parameters were similar in the diet/exercise and pioglitazone groups (Tables 4.5.1 and 4.5.2). After diet/exercise, weight $(11.7 \pm 3.2 \mathrm{~kg})$, body fat $(9.3 \pm 0.9 \mathrm{~kg})$ and visceral fat $(2.5 \pm 0.3$ $\mathrm{kg})$ decreased $(\mathrm{p}<0.001)$. After pioglitazone treatment body weight $2.7 \pm 0.7 \mathrm{~kg}(1.3 \pm 0.4 \mathrm{~kg}$ of fat, both $\mathrm{p}<0.01)$ increased but visceral fat mass did not $(4.4 \pm 0.4 \mathrm{vs} .4 .3 \pm 0.4 \mathrm{~kg})$. Serum triglyceride concentrations decreased after diet/exercise $(1.8 \pm 0.3$ vs. $1.1 \pm 0.1 \mathrm{mmol} / \mathrm{l}$, $\mathrm{p}<0.01)$, but not after pioglitazone $(1.9 \pm 0.2$ vs. $1.8 \pm 0.2 \mathrm{mmol} / \mathrm{l}, \mathrm{p}=0.10)$.

Table 4.5.1. Demographic and body composition before and after intervention

\begin{tabular}{|c|c|c|c|c|c|}
\hline & \multicolumn{2}{|c|}{ Diet/exercise } & \multicolumn{2}{|c|}{ Pioglitazone } & \multirow[b]{2}{*}{ P delta } \\
\hline & Pre & Post & Pre & Post & \\
\hline Age (years) & $41 \pm 2$ & & $36 \pm 2$ & & \\
\hline Weight (kg) & $97.5 \pm 3.3$ & $85.8 \pm 3.1 * * *$ & $98.2 \pm 2.7$ & $100.9 \pm 3.0 * *$ & 0.0001 \\
\hline BMI $\left(\mathrm{kg} / \mathrm{m}^{2}\right)$ & $32.1 \pm 0.7$ & $27.7 \pm 0.8 * * *$ & $33.4 \pm 0.6$ & $34.0 \pm 0.7 *$ & 0.0001 \\
\hline Waist $\varnothing(\mathrm{cm})$ & $105 \pm 2$ & $95 \pm 2 * * *$ & $107 \pm 2$ & $107 \pm 2$ & 0.0001 \\
\hline Hip $\varnothing(\mathbf{c m})$ & $111 \pm 1$ & $105 \pm 2 * * *$ & $115 \pm 1$ & $119 \pm 2 * * *$ & 0.0001 \\
\hline WHR & $0.95 \pm 0.02$ & $0.90 \pm 0.01 * * *$ & $0.94 \pm 0.01$ & $0.90 \pm 0.01 * * *$ & 0.42 \\
\hline$\%$ body fat & $40 \pm 1.4$ & $32 \pm 1.8 * * *$ & $41 \pm 1.4$ & $41 \pm 1.5$ & 0.0001 \\
\hline Body fat (kg) & $35.8 \pm 1.5$ & $26.5 \pm 1.9 * * *$ & $38.0 \pm 1.5$ & $39.3 \pm 1.7 * *$ & 0.0001 \\
\hline FFM (kg) & $59.0 \pm 2.5$ & $58.5 \pm 2.4$ & $58.0 \pm 2.2$ & $59.1 \pm 2.2$ & 0.07 \\
\hline
\end{tabular}

Values $( \pm$ sem) are from the 39 participants that completed both studies. Baseline values are not significantly different between diet/exercise and pioglitazone; ${ }^{*} \mathrm{p}<0.05 * * \mathrm{p}<0.01 * * * \mathrm{p}<0.001$ before vs. after the intervention; $p$ delta signifies the difference between the effect of the two interventions. BMI: body mass index; FFM: fat free mass; WHR: waist-to-hip ratio. 
Table 4.5.2. Effects of diet/exercise and pioglitazone on insulin sensitivity and lipids

\begin{tabular}{lll|lll}
\hline & \multicolumn{3}{c}{ Diet/exercise } & \multicolumn{2}{c}{ Pioglitazone } \\
\cline { 2 - 6 } & pre & post & pre & post & p delta \\
Fasting glucose (mg/dl) & $96 \pm 2$ & $91 \pm 2 * * *$ & $97 \pm 2$ & $92 \pm 2 * *$ & 0.69 \\
Fasting insulin $(\boldsymbol{\mu U} / \mathbf{m L})$ & $8 \pm 1$ & $5 \pm 1 * * *$ & $11 \pm 1$ & $7 \pm 1 * * *$ & 0.06 \\
Fasting C-peptide (nmol/l) & $0.60 \pm 0.04$ & $0.45 \pm 0.03 * * *$ & $0.68 \pm 0.04$ & $0.50 \pm 0.03 * * *$ & 0.61 \\
Serum triglyceride (mg/dL) & $162 \pm 31$ & $102 \pm 12^{* *}$ & $171 \pm 16$ & $156 \pm 16$ & 0.26 \\
HDL-cholesterol (mg/dL) & $39 \pm 2$ & $38 \pm 2$ & $34 \pm 2$ & $35 \pm 2$ & 0.08 \\
Total cholesterol (mg/dL) & $201 \pm 18$ & $159 \pm 7 *$ & $195 \pm 7$ & $190 \pm 7$ & 0.01 \\
Si (mU/l) $)^{-1} \bullet$ min $^{-1}$ & $5.31 \pm 0.97$ & $10.34 \pm 1.88^{* * *}$ & $4.23 \pm 0.61$ & $6.60 \pm 1.09 * *$ & 0.15 \\
\hline
\end{tabular}

Baseline values are not significantly different between diet/exercise and pioglitazone except in fasted insulin, which were higher in the pioglitazone group than in the diet/exercise group $(\mathrm{p}=0.04) .{ }^{*} \mathrm{p}<0.05 * * \mathrm{p}<0.01 * * *$ $\mathrm{p}<0.001$ before vs. after the intervention; $\mathrm{p}$ delta signifies the difference between the effect of the two interventions. Si: Sensitivity index.

Diet/exercise, but not pioglitazone, decreased REE in women (Table 4.5.3); however REE was similar in the diet/exercise and pioglitazone groups, both before and after the interventions. The initial hematocrit in the pioglitazone group averaged at $39.6 \%$ and decreased to $38.2 \%(\mathrm{p}<0.001)$ at the time of the second study. The hematocrit in the diet/exercise group decreased from $39.2 \%$ to $38.7 \%(p=0.06)$.

Table 4.5.3 Resting Energy Expenditure.

\begin{tabular}{lll|ll}
\hline & \multicolumn{2}{c}{ Diet/exercise } & \multicolumn{2}{c}{ Pioglitazone } \\
\cline { 2 - 5 } & pre & post & pre & post \\
Women & $1591 \pm 44$ & $1497 \pm 50^{*}$ & $1580 \pm 60$ & $1612 \pm 56$ \\
Men & $2005 \pm 84$ & $1845 \pm 47$ & $2036 \pm 70$ & $2004 \pm 85$ \\
\hline
\end{tabular}

The mean \pm sem of afternoon resting energy expenditure (Kcal/day) are given for the subjects who completed both studies. ${ }^{*} \mathrm{p}<0.05$ before vs. after the intervention. There were no statistically significant differences between the intervention groups

\section{Glucose kinetics}

Because there were no significant differences in FFM or REE between the diet/exercise and pioglitazone groups before or after the intervention, glucose kinetic values are presented in $\mu \mathrm{mol} / \mathrm{min}$ (Table 4.5.4). However, for easier comparison with other work, glucose flux values are given in $\mu \mathrm{mol} \cdot \mathrm{kg} \mathrm{FFM}{ }^{-1} \cdot \mathrm{min}^{-1}$ below.

In the saline group, neither pioglitazone nor diet/exercise changed glucose flux (preintervention: $11 \pm 1$ and $11 \pm 1$; post-intervention: $11 \pm 1$ and $12 \pm 1 \mu \mathrm{mol} \cdot \mathrm{kg} \mathrm{FFM}^{-1} \cdot \mathrm{min}^{-1}$, respectively) or oxidation (table 4.5.4). Insulin-stimulated glucose disposal increased after diet/exercise ( $30 \pm 2$ vs. $39 \pm 4 \mu \mathrm{mol} \cdot \mathrm{kg} \mathrm{FFM}^{-1} \cdot \mathrm{min}^{-1}$, pre- vs. post-intervention, $\mathrm{P}=0.007$ ), as did glucose oxidation $(\mathrm{P}=0.002)$ and the glucose infusion rate required to maintain euglycemia $(\mathrm{P}=0.004)$.

After pioglitazone, insulin-stimulated glucose disposal increased significantly (from $26 \pm 3$ to $\left.33 \pm 4 \mu \mathrm{mol} \cdot \mathrm{kg} \mathrm{FFM}^{-1} \cdot \mathrm{min}^{-1}, \mathrm{P}=0.03\right)$ whereas glucose oxidation $(\mathrm{P}=0.08)$ and the glucose infusion rate $(\mathrm{P}=0.19)$ increases were not statistically significant. Endogenous glucose production during the insulin clamp was not significantly changed by diet/exercise (pre: $4 \pm 1$ vs. post: $5 \pm 2 \mu \mathrm{mol} \cdot \mathrm{kg} \mathrm{FFM}^{-1} \cdot \mathrm{min}^{-1}, \mathrm{P}=\mathrm{NS}$ ) and was actually greater after the pioglitazone treatment ( $3 \pm 1$ vs. $6 \pm 2 \mu \mathrm{mol} \cdot \mathrm{kg} \mathrm{FFM}^{-1} \cdot \mathrm{min}^{-1}$, pre- vs. post-intervention, $\mathrm{P}=0.03$ ). 


\begin{tabular}{|c|c|c|c|c|}
\hline & \multicolumn{4}{|c|}{ SALINE } \\
\hline & \multicolumn{2}{|c|}{ Diet/exercise } & \multicolumn{2}{|c|}{ Pioglitazone } \\
\hline & pre & post & pre & post \\
\hline Glucose Rd & $730 \pm 132$ & $588 \pm 32$ & $685 \pm 73$ & $644 \pm 65$ \\
\hline Glucose oxidation & $325 \pm 110$ & $337 \pm 88$ & $348 \pm 70$ & $416 \pm 57$ \\
\hline Glucose storage & $405 \pm 179$ & $251 \pm 78$ & $337 \pm 45$ & $227 \pm 79$ \\
\hline \multirow[t]{4}{*}{ FA oxidation } & $311 \pm 23$ & $284 \pm 30$ & $298 \pm 20$ & $285 \pm 20$ \\
\hline & \multicolumn{4}{|c|}{ INSULIN CLAMP } \\
\hline & \multicolumn{2}{|c|}{ Diet/exercise } & \multicolumn{2}{|c|}{ Pioglitazone } \\
\hline & pre & post & pre & post \\
\hline Glucose Rd & $1696 \pm 126$ & $2304 \pm 276^{*}$ & $1466 \pm 154$ & $1852 \pm 250 *$ \\
\hline Glucose oxidation & $849 \pm 61$ & $1120 \pm 70 * *$ & $787 \pm 126$ & $994 \pm 90$ \\
\hline Glucose storage & $847 \pm 127$ & $1184 \pm 281 * *$ & $679 \pm 140$ & $858 \pm 226^{*}$ \\
\hline GIR & $1472 \pm 104$ & $2003 \pm 173 * *$ & $1305 \pm 171$ & $1523 \pm 232$ \\
\hline FA & $156 \pm 16$ & $69 \pm 23 * *$ & $177 \pm 35$ & $117 \pm 19$ \\
\hline
\end{tabular}

Glucose rates of disappearance (Rd) were derived from steady-state tracer turnover data (1330-1400 hrs)).Glucose storage is calculated as glucose uptake minus glucose oxidation.GIR $=$ glucose infusion rate needed to maintain euglycemia. ${ }^{*} \mathrm{p}<0.05, *{ }^{*} \mathrm{p}<0.01$ vs. pre-treatment ( 2 -sided $\mathrm{t}$-test). The differences between the intervention responses were not statistically significant. FA= fatty acid

\section{Oleate kinetics}

Plasma oleate concentrations and SAs for the different groups and different studies are depicted in Figure 4.9 and 4.10. For the saline infused volunteers plasma oleate concentrations averaged $174 \pm 10$ and $166 \pm 13 \mu \mathrm{mol} / \mathrm{L}$ (total FFA concentrations of $536 \pm 28$ and $533 \pm 40$ $\mu \mathrm{mol} / \mathrm{L})$ in the diet/exercise and pioglitazone groups before the interventions. After both diet/exercise and pioglitazone plasma oleate concentrations were essentially unchanged at 174 \pm 8 and $165 \pm 9 \mu \mathrm{mol} / \mathrm{L}$ respectively (total FFA concentrations of $517 \pm 31$ and $527 \pm 30$ $\mu \mathrm{mol} / \mathrm{L})$.

Table 4.5.5 Plasma hormone and catecholamine concentrations

\begin{tabular}{lllll}
\hline & SALINE & & \multicolumn{2}{c}{ INSULIN CLAMP } \\
\hline & Baseline & Post- intervention & Baseline & Post- intervention \\
Insulin (mU/L) & & & & \\
$\begin{array}{l}\text { Diet/exercise } \\
\text { Pioglitazone }\end{array}$ & $5 \pm 1$ & $4 \pm 1^{*}$ & $46 \pm 2$ & $40 \pm 3^{*}$ \\
Growth Hormone ( $\boldsymbol{\mu g} / \mathbf{L})$ & $8 \pm 1$ & $6 \pm 1^{*}$ & $49 \pm 6$ & $36 \pm 3^{*}$ \\
$\begin{array}{l}\text { Diet/exercise } \\
\text { Pioglitazone }\end{array}$ & $1.4 \pm 0.7$ & $2.2 \pm 0.9$ & $1.0 \pm 0.5$ & $1.2 \pm 0.5$ \\
Epinephrine (pmol/L) & $0.9 \pm 0.6$ & $0.4 \pm 0.1$ & $1.8 \pm 1.1$ & $3.1 \pm 0.1$ \\
$\begin{array}{l}\text { Diet/exercise } \\
\text { Pioglitazone }\end{array}$ & $27 \pm 3$ & $23 \pm 4$ & & \\
Norepinephrine (nmol/L) & $25 \pm 6$ & $19 \pm 4$ & $27 \pm 4$ & $25 \pm 4$ \\
$\begin{array}{l}\text { Diet/exercise } \\
\text { Pioglitazone }\end{array}$ & $129 \pm 8$ & $133 \pm 9$ & & \\
\hline
\end{tabular}

Samples were collected at the same time as the samples collected for glucose and FFA kinetics measurements. Pre-intervention values were not significantly different between diet/exercise and pioglitazone *p $<0.05$ vs. before the intervention.

The average oleate concentrations are depicted in figure 4.9; oleate as a percentage of total FFA did not differ between groups or between the pre- and post-intervention studies. Oleate 
flux did not change significantly after either diet exercise $(166 \pm 13$ to $149 \pm 8 \mu \mathrm{mol} / \mathrm{min})$ or pioglitazone ( $159 \pm 14 \mathrm{vs.} 176 \pm 17 \mu \mathrm{mol} / \mathrm{min}$, respectively).

In the insulin clamp group the pre-intervention plasma oleate concentrations averaged $21 \pm 5$ and $33 \pm 5 \mu \mathrm{mol} / \mathrm{L}(\mathrm{P}=\mathrm{NS})$ for diet/exercise and pioglitazone respectively (total FFA concentrations of $70 \pm 16$ and $99 \pm 37 \mu \mathrm{mol} / \mathrm{L})$. After both treatments, oleate concentrations decreased by $\sim 50 \%$ during hyperinsulinemia: $9 \pm 2(\mathrm{P}=0.02$, pre- vs. post- diet/exercise $)$ and $17 \pm 3 \mu \mathrm{mol} / \mathrm{L}(\mathrm{P}=0.13$, pre- vs. post-pioglitazone). The corresponding FFA concentrations were $33 \pm 5$ and $60 \pm 11$ $\mu \mathrm{mol} / \mathrm{L}$ in the diet/exercise group and pioglitazone group.

In the diet/exercise group, oleate flux during the insulin clamp decreased from $33 \pm 5 \mu \mathrm{mol} / \mathrm{min}$ preintervention to $18 \pm 4$ $\mu \mathrm{mol} / \mathrm{min}$ post-intervention $(\mathrm{P}=0.03)$. In the pioglitazone group, oleate flux was $39 \pm 9$ and $39 \pm 6$ $\mu \mathrm{mol} / \mathrm{min}$ before and after the intervention respectively.

Because of the discordant changes in oleate concentrations and fluxes we analyzed oleate clearance changes in the two groups. The repeated measures ANOVA indicated an overall significant $(\mathrm{P}=0.006)$ between group (diet/exercise vs. pioglitazone) difference in oleate clearance. By ANOVA the change in clearance between diet/exercise and pioglitazone groups was statistically significant for both the saline control participants $(\mathrm{P}=0.02)$ and the insulin clamp participants
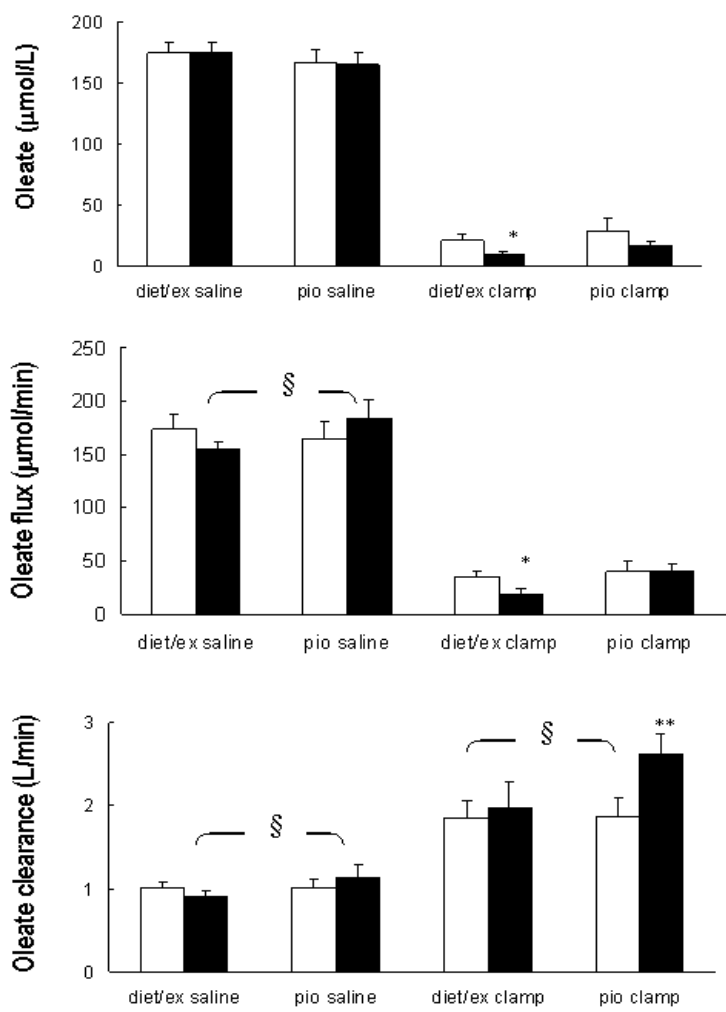

Figure 4.9 Effect of Diet/ex vs. PIO on oleate concentration, flux and clearance during saline and clamp conditions. Preintervention values are depicted by open bars, post-intervention values by solid bars. * $\mathrm{P}<0.05 ;{ }^{*} \mathrm{P}<0.01$ vs. baseline; $\S \mathrm{P}<0.05$ between interventions. In the pioglitazone treated group oleate clearance increased during the saline infusion ( $\mathrm{P}=0.05$, paired $\mathrm{t}$-test) from pre- to post-intervention. $(\mathrm{P}=0.003)$. Preintervention oleate clearance in the saline control studies was $0.97 \pm 0.07 \mathrm{~L} / \mathrm{min}$ in the diet/exercise and $0.97 \pm 0.10 \mathrm{~L} / \mathrm{min}$ in pioglitazone group (Figure 4.9). Post-intervention oleate clearance decreased somewhat after diet/exercise $(0.88 \pm 0.20 \mathrm{~L} / \mathrm{min})$, but increased after pioglitazone $(1.09 \pm 0.13 \mathrm{~L} / \mathrm{min})$. 
Similarly, in the insulin clamp groups, oleate clearance did not change significantly in response to diet/exercise $(1.79 \pm 0.20$ vs. $1.92 \pm 0.30 \mathrm{~L} / \mathrm{min}, \mathrm{p}=\mathrm{NS}$, Figure 4.9$)$, but, in contrast, increased from $1.80 \pm 0.21$ to $2.52 \pm 0.24 \mathrm{~L} / \mathrm{min}(\mathrm{p}<0.01)$ in response to pioglitazone.

\section{Plasma triglyceride radioactivity}

The concentration of ${ }^{3} \mathrm{H}$ triglyceride $(\mathrm{dpm} / \mathrm{mL})$ in plasma during the post-intervention study was virtually identical between groups ( $\mathrm{P}=0.98$ by ANOVA), averaging $964 \pm 92$ and $994 \pm$ 264 in the diet/exercise and pioglitazone groups during the saline condition, and $1009 \pm 177$ and $916 \pm 139$ in the diet/exercise and pioglitazone groups during the insulin clamp.

\section{Discussion}

We assessed the effects pioglitazone on overnight postabsorptive and insulin-regulated NEFA and glucose metabolism in non-diabetic upper body obese men and women. A diet/exercise group was also studied to allow us to compare two insulin-sensitizing regimens. To the extent that better regulation of NEFA concentrations has beneficial effects on the metabolic complications of upper body obesity, understanding whether and how these two regimens alter fatty acid metabolism may provide insights into mechanisms of health improvement.

After diet/exercise, the ability of insulin to suppress lipolysis was enhanced, resulting in $\sim 50 \%$ lower oleate concentrations during hyperinsulinemia. In contrast, after pioglitazone treatment the $\sim 50 \%$ lower plasma oleate concentrations during the insulin clamp were due to a $40 \%$ increase in clearance rather than reduced lipolysis.

We found no previous reports that pioglitazone increases NEFA clearance in humans, although increased NEFA clearance was seen during lipid emulsion infusion into thiazolidinedione treated animals (290). Previous studies suggested that TZDs lower NEFA concentrations in type 2 diabetes via improved insulin suppression of lipolysis $(259,282-284)$.

In the current study of non-diabetic upper body obese adults we did not find greater insulin suppression of lipolysis. It is possible that the improved glycaemic control achieved with thiazolidinedione treatment of type 2 diabetes secondarily improves insulin suppression of lipolysis, whereas a primary effect of TZDs is enhanced removal of NEFA from the circulation.

Another possible explanation for this discrepancy might be the use of glycerol kinetics rather than NEFA flux as a reflection of adipose tissue lipolysis in most of the studies (259). Glycerol $\mathrm{Ra}$ is a reflection of both adipose tissue lipolysis and the intravascular hydrolysis of VLDL by lipoprotein lipase (291).

We found indirect evidence for enhanced insulin regulation of overnight postabsorptive lipolysis by both interventions; oleate release in the saline infused volunteers was not increased in either the diet/exercise or pioglitazone groups despite significantly reduced insulin concentrations.

Lipolysis is exquisitely sensitive to insulin $(292,293)$, such that NEFA flux should have increased in our volunteers had there been no change in insulin's antilipolytic effects. The absence of this change implies that, at least at basal insulin concentrations, insulin was more effective at suppressing lipolysis.

Given the sensitivity of lipolysis to insulin regulation, one might question how our insulin clamp insulin clamp results relate to the daily fluctuations in NEFA availability.

The plasma insulin concentrations we achieved during the insulin clamp are similar to those we have seen in upper body obese individuals following ingestion of a mixed meal (38). In that same study (38) we found the postprandial nadir of endogenous oleate concentrations were similar to the steady-state suppressed levels we observed during this study. 
These insulin concentrations discriminate between upper body and lower body obesity as regards suppression of lipolysis $(38,43)$, whereas lower doses of insulin do not (37). In addition, the dose given in this study is identical to that we have found to discriminate nicely between upper body obese and non-obese men and women as regards glucose and NEFA metabolism (Sheehan et al, Obesity Research 11 (Suppl): A53, 2003).

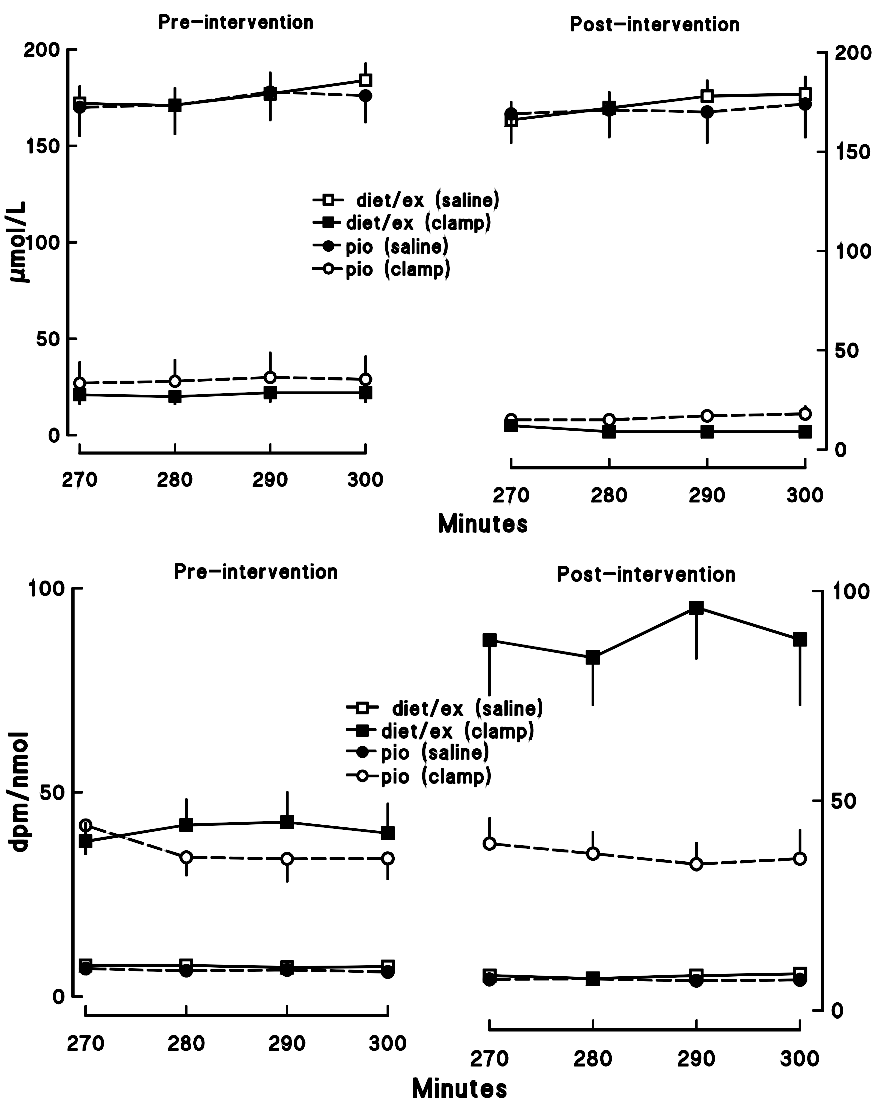

Figure 4.10 Plasma concentrations(upper panel) and specific activities (lower panel) of oleate over the last 30 min of the insulin clamp or control saline infusion in volunteers treated with diet/exercise (diet/ex) or pioglitazone (pio). Values obtained prior to beginning treatment (Pre-intervention) are show on the left and those observed after treatment (Post-intervention) are shown on the right.
Finally, the NEFA concentrations seen during hyperinsulinemia following diet/exercise or pioglitazone are more in keeping with the nadir endogenous NEFA concentrations seen following meal ingestion in non-obese and lower body obese adults (38). Because of the importance of insulin suppression of NEFA concen-trations in regulating glucose metabolism (294), we believe that the dose of insulin we selected is relevant to the effects of diet/exercise and pioglitazone on postprandial metabolism.

These studies do not address the tissue(s) or the mechanism responsible for the greater NEFA clearance, although we can virtually exclude increased uptake into tissues that use NEFA for immediate oxidative needs.

Increased NEFA uptake into muscle would have

to be accompanied by increased fatty acid oxidation in order to prevent accumulation of intramyocellular fatty acids, which are thought to result in insulin resistance. Fatty acid oxidation was not increased following pioglitazone treatment in either the saline or in insulin clamp studies (Table 4.5.4), which should have been detected if skeletal muscle were the primary site of increased NEFA clearance. Because fatty acid oxidation did not increase, this indicates that pioglitazone increased non-oxidative NEFA clearance. One tissue that might account for 
this would be liver, which could then export the NEFA's as VLDL-triglyceride back to adipose tissue.

Adipose tissue lipoprotein lipase (LPL) can act on VLDL triglyceride (295), so that circulating VLDL triglyceride (296) can return to adipose tissue via a form of "tissue substrate cycling". A recent report showed that pioglitazone treatment of type 2 diabetes lowered VLDLtriglyceride concentrations, did not increase hepatic VLDL-triglyceride production, but did increase VLDL-triglyceride clearance (297). Whether the same effects occur in non-diabetic individuals is unknown. Serum triglyceride concentrations did not decrease in our group. Thus, if VLDL-triglyceride clearance increased in this study, VLDL-triglyceride production must have increased also. Another possible route of NEFA clearance might be direct uptake into adipose tissue, although the lack of NEFA tracer uptake across adipose tissue (298) argues against this possibility. Either of these processes could allow increased non-oxidative NEFA clearance without predisposing to ectopic fat deposition.

Both diet/exercise and pioglitazone treatment interventions improved peripheral insulin action as assessed by fasting glucose insulin and C-peptide concentrations, Si from an intravenous glucose tolerance test (288), and glucose Rd during a euglycaemic hyperinsulinemic clamp. Surprisingly, endogenous glucose production was greater during the insulin clamp after pioglitazone treatment, albeit this was in the context of $25 \%$ lower plasma insulin concentrations.

This response of endogenous glucose production to TZDs has previously been reported to occur in dogs (299) and humans (300) despite increased peripheral insulin sensitivity. It was speculated (300) that this phenomenon is due to a TZDs induced increase in insulin clearance (which is consistent with our data) because endogenous glucose $\mathrm{Ra}$ is quite sensitive to small changes in plasma insulin concentrations. It should be noted that some investigators have found greater suppression of endogenous glucose production following pioglitazone treatment of type 2 diabetes during a $40 \mathrm{mU} / \mathrm{min} \mathrm{m}^{-2}$ dose insulin clamp (281). Whether our results differ because of the population (non-diabetics) or the differences in insulin infusion rates is unclear.

The 6 hour ${ }^{3} \mathrm{H}$-oleate infusion used in our study resulted in incorporation of the NEFA tracer into circulating triglycerides. The VLDL triglycerides are thought to be taken up in peripheral tissues via LPL mediated hydrolysis (295). If some of the fatty acids from circulating triglycerides escape uptake by tissues following hydrolysis and enter the circulating NEFA pool, this would create a second source of ${ }^{3} \mathrm{H}$ oleate (in addition to the tracer being infused). Because the NEFA turnover calculation assumes a single, externally infused source of ${ }^{3} \mathrm{H}$ oleate an "escape" of ${ }^{3} \mathrm{H}$ oleate from VLDL triglyceride could cause an underestimation of oleate flux. This would be a special problem for interpreting our clearance data from the post-intervention study if there were different amounts of radiolabeled triglycerides in plasma between the groups. The finding that plasma ${ }^{3} \mathrm{H}$ triglyceride concentrations were virtually identical between groups implies that, if this process occurs, its effect would be equal in each group. Thus, our conclusion that NEFA clearance increases with pioglitazone is not confounded by differences in plasma ${ }^{3} \mathrm{H}$ triglyceride concentrations.

In summary, in this study we examined the effects of pioglitazone vs. diet/exercise treatment on the insulin regulation of NEFA and glucose metabolism in upper body obese, non-diabetic adults. Both interventions improved insulin sensitivity with respect to glucose metabolism, although diet/exercise resulting in an average $9 \mathrm{~kg}$ fat loss appears to be somewhat better than pioglitazone. Pioglitazone increased NEFA clearance most dramatically during physiological hyperinsulinemia, resulting in reduced NEFA concentrations without reducing lipolysis. The greater NEFA clearance is unlikely to be due to insulin sensitization per se, 
because it was not seen in the diet/exercise group. This action of TZDs may be important in their ability to improve glucose metabolism in type 2 diabetes. 


\title{
4.5.4 Diet/Exercise versus Pioglitazone: Effects of Insulin Sensitization with Decreasing or Increasing Fat Mass on Adipokines and Inflammatory Markers
}

\author{
Samyah Shadid ${ }^{1}$, Coen DA Stehouwer ${ }^{2}$ and Michael D. Jensen, J Clin Endo- \\ crinol Metab. 2006 Sep;91(9):3418-25. Epub 2006 Jun 27 \\ Endocrine Research Unit, Mayo Clinic, Rochester, Minnesota, USA \\ ${ }^{1}$ Current address: VieCuri Medical Center, Venlo, The Netherlands \\ ${ }^{2}$ Department of Medicine, University Hospital Maastricht, Maastricht, \\ The Netherlands
}

\begin{abstract}
Background: Plasma adipokine concentrations are variably related to fatness/insulin resistance and may act via endocrine mechanisms. We assessed the relationship amongst plasma adipokine concentrations and their relationship with insulin sensitivity and body composition in obese adults before and after insulin sensitization accomplished using diet/exercise or pioglitazone.

Methods: Plasma adipokine concentrations, insulin sensitivity and body composition were assessed in 39 upper body obese insulin resistant, non-diabetic adults before and after 19 weeks of diet/exercise or $30 \mathrm{mg} /$ day pioglitazone.

Results: Diet/exercise reduced body fat and visceral fat and improved insulin sensitivity parameters; pioglitazone improved insulin sensitivity to a similar degree but increased body fat. Adiponectin increased more after pioglitazone $(4770 \pm 487 \mathrm{vs} .8351 \pm 693.6 \mathrm{ng} / \mathrm{ml}, \mathrm{p}<0.001)$ than after diet/exercise (4704 \pm 367 to $5426 \pm 325.3 \mathrm{ng} / \mathrm{ml}, \mathrm{p}<0.01)$, whereas TNF- $\alpha$, IL-6 and resistin did not change. CRP decreased with diet/exercise. Adipokine concentrations were not correlated with each other at baseline or after insulin sensitization, except TNF- $\alpha$ and IL-6 $(\mathrm{r}=0.43, \mathrm{p}<0.05)$; IL-6 was inversely correlated with resistin. Only adiponectin was correlated $(p<0.05)$ with indices of insulin sensitivity. Adiponectin concentrations were inversely correlated with visceral fat and with subcutaneous fat depots in men, but positively correlated with subcutaneous fat in women.

Conclusion: Plasma adipokine concentrations were not consistently interrelated and only adiponectin displayed the expected relationship with insulin sensitivity and sensitization. These findings do not support an endocrine role for resistin, TNF- $\alpha$ and IL- 6 in mediating changes in insulin resistance after diet/exercise or pioglitazone.
\end{abstract}




\section{Introduction}

In addition to triglyceride storage, adipose tissue produces several hormones (leptin, resistin, adiponectin) and cytokines (interleukin-6 (IL-6), tumor necrosis factor- $\alpha$ (TNF- $\alpha$ )). The production of these so-called 'adipokines' mostly parallels fat mass; only adiponectin concentrations are inversely correlated with fatness. Some adipokines have been linked to insulin resistance whereas others, such as TNF- $\alpha$ and the interleukins $(301,302)$ stimulate acute phase proteins such as $\mathrm{C}$-reactive protein (CRP).

There remain uncertainties surrounding the exact role of adipokines in the pathophysiology of insulin resistance and obesity. Are they epiphenomena of obesity and insulin resistance or do they act to modulate insulin action via endocrine mechanisms? Are they primarily related to fatness, body fat distribution and/or to insulin resistance? If these molecules act through an endocrine role then developing approaches to enhance or block their production or action would be a therapeutic goal. In addition, understanding the relationship of these adipokines and inflammatory markers to insulin resistance and adiposity will help determine whether assaying their blood concentrations offers important clinical information.

We conducted this study to assess the suitability of several adipokines/cytokines as possible markers for insulin resistance and changes in insulin resistance; changes in insulin resistance were induced by either diet and exercise or pioglitazone. Strong associations might support an endocrine function whereas the absence of associations would argue against an endocrine role. We also wished to understand the inter-relationships between the adipokines/cytokines to determine if there are a limited few measures that can serve as surrogates for the remaining adipokines.

\section{Methods}

\section{Subjects and Study Protocol}

Data from the 39 participants described in Chapter 4.5.2 were analyzed. The volunteers underwent blood testing (plasma adipokine concentrations, complete blood count, chemistry panel, lipid profile), an intravenous glucose tolerance test (Bergman's minimal model $(270,271)$ ), abdominal CT and DEXA scanning (both see Chapter 4.5.2.3). Fat biopsies were taken from femoral and abdominal subcutaneous depots.

Afterwards, subjects were randomized to receive $30 \mathrm{mg}$ of PIO daily (10 men, 10 women) or a diet/exercise program (10 men, 9 women) for 18-20 weeks as described (Chapter 4.5.2.), after which baseline studies were repeated. Assessment of fat compartment volume, fat cell size and specific assays are also described in Chapter 4.5.2.

\section{Statistics}

Statistical analyses were done using SPSS 11.5: paired t-tests for comparisons between before and after treatment, and $2 \times 2$ repeated measures ANOVA with factors for group (diet/exercise vs. pioglitazone) and time (the repeated measure - before and after intervention) for statistical comparisons of the two groups (PIO vs. diet/exercise) and their responses to the interventions. Values are expressed as mean \pm standard error of the mean; $\mathrm{P}$ values $<0.05$ were considered statistically significant. Because performing multiple correlations on large amounts of data increases the likelihood of drawing erroneous conclusions, we performed a Principal Component Analysis (PCA) on the baseline data with a Varimax Rotation and Kaiser Normalization. The goal was to identify which factors were sex-dependent and which correlated with insulin sensitivity and/or fat depots. This type of factor analysis is used for data 
reduction and works by uncovering the latent structure (dimensions) of a set of variables. It does not assume a specified dependent variable, but simply examines which data cluster together by seeking the 'best fit'.

Bivariate Pearson's correlations were used to assess whether changes in plasma adipokine concentrations were associated with treatment-induced changes in other parameters. If parameters were identified as sex-dependent in the PCA, we either performed partial correlations (correcting for sex), or present bivariate correlations for both genders separately.

If hormone/adipokine concentrations or changes herein were not normally distributed we repeated the correlation analyses after logarithmic transformation. If any of the changes in concentration were negative values and the data were not normally distributed we allowed for log transformation assuming that $\log ($ after-before $)=\log ($ after/before $)=\log$ (after) $-\log$ (before). This was especially the case for TNF- $\alpha$ values and the post-diet/ex change herein; log transformations are only explicitly mentioned in the text if they influenced the outcome of the results.

Table 4.5.6 Effect of insulin sensitization on anthropometric characteristics and adipokine concentrations

\begin{tabular}{lllll}
\hline & \multicolumn{3}{l}{ Diet/exercise } & \multicolumn{2}{l}{ Pioglitazone } & \\
\cline { 2 - 5 } & pre & post & pre & post \\
\hline Age $($ years) & $41 \pm 2$ & & $36 \pm 2$ & \\
BMI $\left(\mathrm{kg} / \mathrm{m}^{2}\right)$ & $32.1 \pm 0.7$ & $27.7 \pm 0.8^{* * *}$ & $33.4 \pm 0.6$ & $34.0 \pm 0.7^{*} \S$ \\
Fat $(\%)$ & $40 \pm 1.4$ & $32 \pm 1.8^{* * *}$ & $41 \pm 1.4$ & $41 \pm 1.5 \S$ \\
Resistin $(\mathrm{ng} / \mathrm{ml})$ & $9.4 \pm 1.2$ & $7.7 \pm 0.9$ & $8.9 \pm 1.0$ & $8.1 \pm 1.0$ \\
Adiponectin $(\mathrm{ng} / \mathrm{ml})$ & $4704 \pm 367$ & $5426 \pm 325^{* *}$ & $4770 \pm 487$ & $8351 \pm 693.6^{* * *} \S$ \\
CRP $(\mathrm{mg} / \mathrm{dl})$ & $0.22 \pm 0.04$ & $0.12 \pm 0.02^{* *}$ & $0.30 \pm 0.09$ & $0.22 \pm 0.05$ \\
TNF- $\alpha(\mathrm{pg} / \mathrm{ml})$ & $1.2 \pm 0.1$ & $2.3 \pm 0.7$ & $1.4 \pm 0.1$ & $1.6 \pm 0.3$ \\
IL-6 $(\mathrm{pg} / \mathrm{ml})$ & $1.7 \pm 0.2$ & $1.9 \pm 0.2$ & $2.1 \pm 0.2$ & $1.9 \pm 0.3$ \\
\hline
\end{tabular}

$\mathrm{BMI}=$ Body Mass Index; CRP=C-reactive protein; $\mathrm{TNF}=$ tumor necrosis factor; IL-6= interleukin-6; WHR= Waist-to-hip ratio. There were no differences in baseline values between the two intervention groups. ${ }^{*} \mathrm{p}<0.05$, ${ }^{* *} \mathrm{p}<0.01, * * * \mathrm{p}<0.001$ before vs. after intervention. $\S \mathrm{p}<0.001$ effect of diet/exercise vs. pioglitazone.

\section{Results}

\section{Baseline characteristics and adipokine relationships}

As reported (303), the diet/exercise and PIO groups were well matched for age, BMI, body composition, and insulin resistance parameters (Table 1). The baseline Si values ranged from 0.71 to $19.9(\mathrm{mU} / 1)^{-1} \bullet \mathrm{min}^{-1}$, fasting glucose from $97-117 \mathrm{mg} / \mathrm{dl}$, fasting insulin from $2.6-$ $23.0 \mu \mathrm{U} / \mathrm{mL}$ and fasting C-peptide from $0.35-1.09 \mathrm{nmol} / \mathrm{l}$. The groups also had similar preintervention adipokine concentrations (Table 4.5.6).

The PCA identified adiponectin and CRP as sex-dependent variables (Table 4.5.7); adiponectin and CRP concentrations were greater in women than in men: $5736 \pm 471$ vs. 3838 $\pm 280 \mathrm{ng} / \mathrm{ml}(\mathrm{p}=0.001)$ and $0.37 \pm 0.09$ vs. $0.17 \pm 0.03 \mathrm{mg} / \mathrm{dl}(\mathrm{p}=0.04)$ respectively.

Adiponectin was the only adipokine that clustered statistically with fasting plasma insulin, fasting C-peptide and Si (component 1). The factor (component) loadings are the correlation coefficients between the variables (rows) and factors (columns) and are the basis for imputing a label to the different factors. Loadings above .6 are usually considered "high" and those below .4 are "low." If sex was eliminated as a factor in the model CRP, TNF- $\alpha$ and IL- 6 clustered together (component 2), but they did not cluster with insulin sensitivity parameters. 
Table 4.5.7 Principal Component Analysis with Varimax Rotation.

\begin{tabular}{lcc}
\hline & \multicolumn{2}{c}{ Component } \\
\hline Total variance explained & $\mathbf{1}$ & $\mathbf{2}$ \\
& 38.3 & 22.0 \\
Gender & & $\mathbf{0 . 8 0}$ \\
Resistin & -0.14 & 0.01 \\
Adiponectin & -0.08 & $\mathbf{0 . 7 7}$ \\
C-Reactive Protein & $\mathbf{- 0 . 4 2}$ & $\mathbf{0 . 6 6}$ \\
Tumor Necrosis Factor- $\alpha$ & 0.27 & 0.07 \\
Interleukin 6 & 0.03 & 0.18 \\
Fasting C-peptide & 0.24 & -0.05 \\
Fasting glucose & $\mathbf{0 . 9 1}$ & -0.17 \\
Fasting insulin & 0.27 & -0.08 \\
Si & $\mathbf{0 . 9 2}$ & 0.08 \\
\hline
\end{tabular}

Component 1 and 2 represent the 'best and second best fit' of the variables presented in this table. All of the components combined explain $38.3 \%$ (component 1 ) and $22.0 \%$ (component 2 ) of the variance. In component 1 , adiponectin is the only adipokine to cluster (inversely) with insulin sensitivity parameters. Component 2 indicates that adiponectin and CRP, but not the other adipokines, cluster with gender. $\mathrm{Si}=$ Insulin sensitivity index.

\section{Baseline correlations}

\section{Adiponectin}

For men and women combined, baseline adiponectin concentrations were positively correlated with $\mathrm{Si}$ and inversely correlated with fasting plasma insulin and C-peptide concentrations (table 4.5.8).

Table 4.5.8. Baseline bivariate adipokine correlations

\begin{tabular}{|c|c|c|c|c|c|c|c|c|c|}
\hline & Resistin & TNF- $\alpha$ & IL-6 & Adipor & ctin & & CRP & & \\
\hline & all subjects & all & all & all & $q$ & 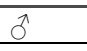 & all & 운 & 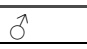 \\
\hline Resistin & 1 & -0.05 & $-0.39 *$ & -0.06 & & & -0.20 & & \\
\hline Adiponectin & & -0.02 & 0.05 & 1 & & & 0.28 & & \\
\hline C-Reactive protein & & $0.34 *^{1}$ & $0.31 *^{1}$ & & & & 1 & & \\
\hline TNF $-\alpha$ & & 1 & $0.43 *$ & & & & & & \\
\hline $\mathrm{Si}$ & 0.28 & -0.02 & -0.10 & $0.33^{*}$ & 0.39 & 0.37 & -0.12 & $-0.25^{2}$ & -0.32 \\
\hline F-glucose & -0.14 & -0.21 & -0.08 & -0.26 & -0.33 & 0.05 & -0.14 & -0.13 & 0.08 \\
\hline F-insulin & -0.16 & 0.01 & 0.27 & $-0.38 *$ & -0.15 & $-0.53 *$ & 0.16 & 0.16 & $0.72 * *$ \\
\hline F-C-peptide & -0.10 & -0.04 & 0.23 & $-0.36^{*}$ & -0.26 & -0.37 & 0.15 & 0.15 & $0.65^{* *}$ \\
\hline Fat $\%$ & 0.08 & 0.31 & $0.33 *$ & $0.39^{*}$ & 0.29 & -0.38 & $0.41 *$ & 0.24 & 0.43 \\
\hline Total FAT (kg) & 0.05 & 0.15 & 0.20 & 0.16 & $0.55^{*}$ & $-0.53^{*}$ & $0.32 *$ & 0.30 & 0.39 \\
\hline UBNV fat (kg) & 0.08 & 0.07 & 0.19 & 0.20 & 0.15 & $-0.54 *$ & 0.19 & 0.21 & 0.31 \\
\hline Leg fat (kg) & 0.06 & 0.30 & 0.27 & $0.36^{*}$ & 0.39 & -0.32 & $0.46 * *$ & 0.03 & 0.32 \\
\hline Visceral fat (kg) & -0.08 & 0.07 & -0.11 & $-0.40 *$ & $0.54 *$ & $-0.53 *$ & -0.04 & 0.41 & 0.33 \\
\hline
\end{tabular}

$* \mathrm{p}<0.05, * * \mathrm{p}<0.01$. $\mathrm{F}=$ fasted. UBNV= upper body non-visceral. $\mathrm{Si}=$ Insulin Sensitivity Index. ${ }^{1} \mathrm{r}=0.27(\mathrm{p}=0.11)$ and $0.28(\mathrm{p}=0.12)$ for TNF- $\alpha$ and IL-6 respectively when corrected for gender.

As expected, there was a positive correlation between adiponectin and $\mathrm{Si}$ and a negative correlation with visceral fat for men and women considered together, however, body fat com- 
partments were correlated positively with adiponectin in women and negatively with adiponectin in men (Figure 4.11).

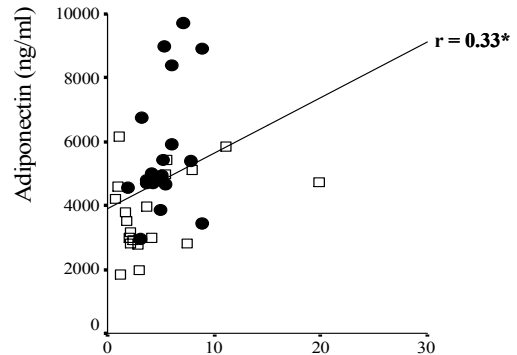

$\mathrm{Si}((\mathrm{mU} / \mathrm{l})-1 . \mathrm{min}-1$

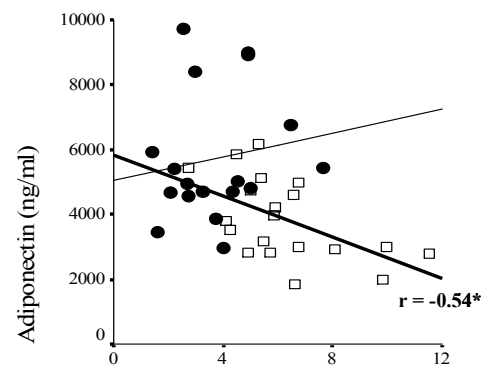

Visceral fat $(\mathrm{kg})$

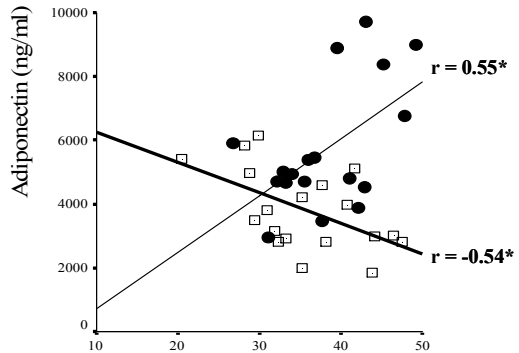

total fat $(\mathrm{kg})$

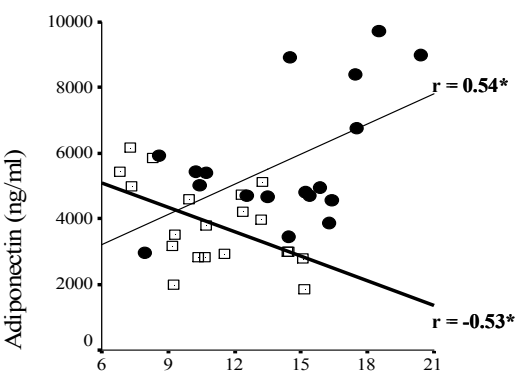

Leg fat $(\mathrm{kg})$

Figure 4.11. Association of adiponectin with insulin sensitivity and body fat Closed circles represent women, open diamonds men. $\mathrm{Si}=$ insulin sensitivity index $\left((\mathrm{mU} / \mathrm{l})^{-1} \bullet \mathrm{min}^{-1}\right)$. Adiponectin correlates with $\mathrm{Si}$ in the complete group $(\mathrm{r}=0.33, \mathrm{p}<0.05)$, but this is just short of reaching statistical significance in men $(r=0.37, p=0.11)$ and women $(r=0.39, p=0.11)$ separately. Adiponectin correlations with the various fat compartments are inverse (as expected) in men, but positive in women. In the latter, visceral fat is not correlated with adiponectin $(\mathrm{r}=0.15, \mathrm{p}=\mathrm{NS}) .{ }^{*} \mathrm{p}<0.05$

\section{Resistin}

Plasma resistin concentrations did not correlate with percent body fat, fat compartments, insulin sensitivity parameters or the other adipokines (Table 4.5.8). To the extent that there were trends for associations between resistin and other parameters, the correlations were negative with IL-6 and positive with $\mathrm{Si}(\mathrm{p}=0.09)$.

\section{CRP}

The correlation coefficients between plasma CRP and other adipokines are given in table 4.5.8. Given the small number of men and women in each group the statistical power is limited and it is difficult to draw conclusions regarding the lack of certain associations. We did find that CRP concentrations were inversely correlated with adiponectin in men, and that CRP and TNF- $\alpha$ were positively correlated in women. 
Interleukin-6 and TNF- $\alpha$

Baseline IL-6 correlated with TNF- $\alpha$ and percent body fat, but not with other adipokines, insulin sensitivity parameters or regional fat compartments. Il-6 correlated with CRP only if the values from the person with the highest CRP concentrations were excluded or if log transformation was performed $(\mathrm{r}=0.52, \mathrm{p}=0.001)$.

Plasma TNF- $\alpha$ concentrations did not correlate with insulin sensitivity parameters or other adipokines at baseline, except Il-6 (table 4.5.8). The correlations between plasma TNF- $\alpha$ concentrations and percent body fat and leg fat did not reach statistical significance $(p=0.07$ and 0.08 respectively). Plasma TNF- $\alpha$ concentrations were positively correlated with abdominal, but not femoral fat cell size (Figure 4.12). Log transformation of the data did not alter these outcomes; none of other adipokine concentrations were correlated with fat cell size.

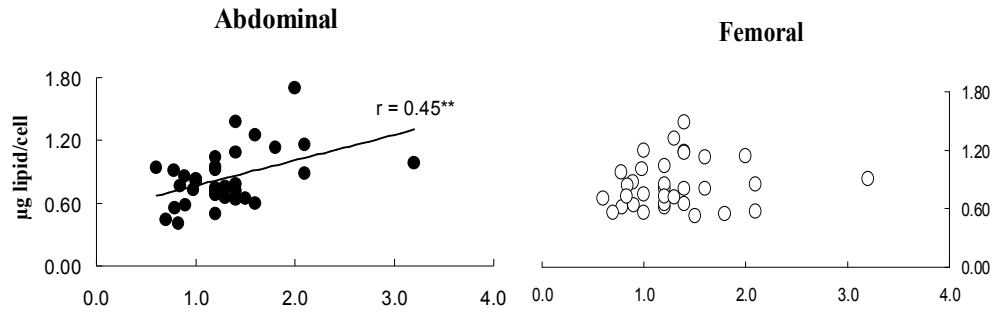

Figure 4.12. Correlation between TNF- $\alpha$ concentration and fat cell size

\section{Response to interventions}

Volunteers in the diet/exercise group lost $11.8 \pm 1.1 \mathrm{~kg}$ of weight, $2.5 \pm 0.3 \mathrm{~kg}$ of visceral fat, $3.4 \pm 0.7 \mathrm{~kg}$ of upper body non-visceral fat and $2.9 \pm 0.3 \mathrm{~kg}$ of leg fat $(\mathrm{p}<0.001)$, but a nonsignificant amount of FFM $(-0.5 \pm 0.6 \mathrm{~kg})$. The PIO group gained weight $(2.7 \pm 0.7 \mathrm{~kg})$ and fat $(1.3 \pm 0.4 \mathrm{~kg}$, both $\mathrm{p}<0.01)$, predominantly in the leg depot $(1.0 \pm 0.2 \mathrm{~kg}, \mathrm{p}<0.001)$; visceral fat was unchanged (303).

Both interventions significantly improved all insulin sensitivity parameters and there were no significant differences between the effects of PIO and diet/exercise (303). Diet/exercise decreased abdominal fat cell size more so than femoral fat cell size, whereas PIO, oppositely, decreased femoral more than abdominal adipocyte size (303).Plasma concentrations of resistin, TNF- $\alpha$ and IL-6 were not significantly altered by either intervention (table 4.5.6). The increase in adiponectin concentrations was greater $(\mathrm{p}<0.001)$ after pioglitazone than diet/exercise.

\section{Correlations after insulin sensitization}

\section{Resistin}

The change in resistin concentrations after diet/exercise correlated with the changes in insulin $(\mathrm{r}=-0.61, \mathrm{p}<0.01)$ and adiponectin (men only, $\mathrm{r}=0.80, \mathrm{p}=0.005)$, but in directions opposite to the expectation. 
The change in resistin concentrations correlated with the change in adiponectin concentration $(\mathrm{r}=-0.69, \mathrm{p}<0.01)$ for the pioglitazone group data; all others resistin associations were either in the opposite direction (total body and leg fat: $\mathrm{r}=-0.53$ and -0.59 respectively, both $\mathrm{p}<0.05$ ) or absent (changes in insulin sensitivity parameters, body composition and other adipokines).

\section{Adiponectin}

In the diet/exercise group, there were no statistically significant correlations between changes in adiponectin and changes in insulin sensitivity parameters in men or women. In contrast, the changes in adiponectin concentrations were inversely correlated with changes in total fat in men $(p=0.03)$; the correlation with changes in leg $(p=0.07)$ and visceral fat $(p=0.06)$ did not reach statistical significance.

In women, the correlations between adiponectin concentrations and changes in body fat were positive (figure 4.13), but weak and non-significant $(r=0.18,0.32$ and 0.42 for total, leg and visceral fat respectively).

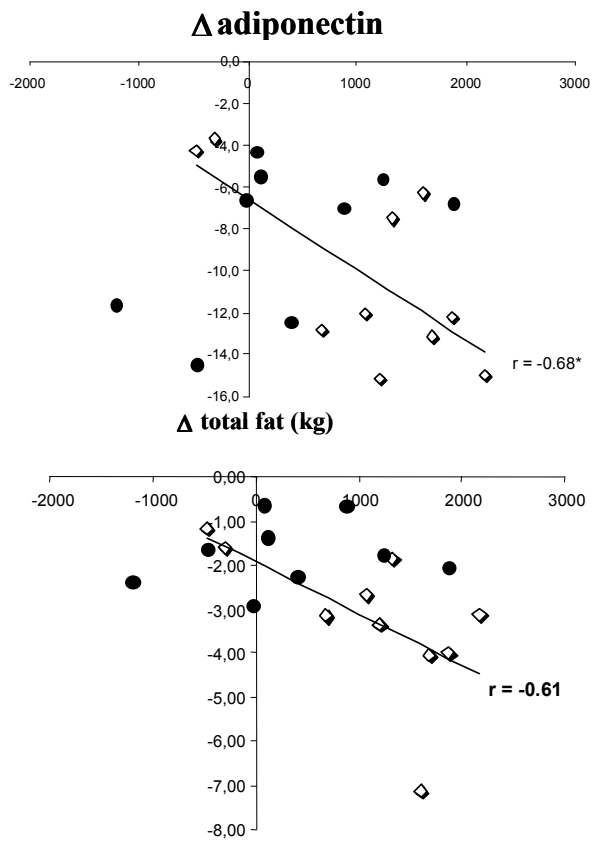

$\Delta$ visceral fat $(\mathrm{kg})$
Figure 4.13 Sex difference in postdiet/exercise correlations between changes in adiponectin and fat depots. Closed circles represent women, open diamonds men. Correlations of the postdiet/exercise change in adiponectin with changes in all fat compartments presented are inverse in men (as expected), but positive in women. $* \mathrm{p}<0.05$

- women $\diamond$ men $* \mathrm{p}<0.05$

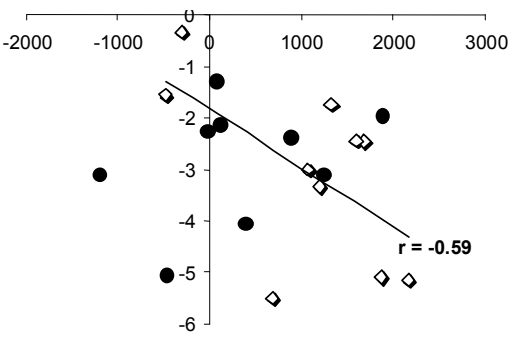

$\Delta$ leg fat (kg)

For the pioglitazone treated group, the adiponectin concentration changes were not significantly correlated with changes in insulin sensitivity or body composition in men, women or the combined group.

\section{CRP, Interleukin- 6 and $T N F-\alpha$}

The change in CRP concentrations correlated significantly with the change in total body fat in the diet/exercise group $(\mathrm{r}=0.53, \mathrm{p}=0.03)$. Changes in IL-6 and TNF- $\alpha$ after either diet/exercise or pioglitazone were not correlated with changes in other inflammatory markers, adipokine concentrations, insulin sensitivity parameters or body composition, with the exception of a post-pioglitazone correlation between the change in IL-6 and leg fat $(r=0.48, p<0.05)$. 


\section{Discussion}

We recently compared the effects of a diet/exercise weight loss program with pioglitazone on body fat compartments and insulin sensitivity in upper body obese adults (303). There has subsequently been considerable interest in the possible endocrine role of the adipokines/inflammatory markers as they relate to insulin sensitivity and body fat.

Because weight loss and pioglitazone increased insulin sensitivity to a comparable degree, but had opposite effects on adipose tissue mass, we took advantage of samples collected during the course of this study (303) to assess the relationship between adipokines, insulin resistance and body fat characteristics, and to try to distinguish fat mass effects from insulin sensitizing effects. We also assessed whether changes in insulin resistance and body fat were related to changes in plasma adipokine concentrations. Strong correlations between circulating adipokine concentrations and insulin sensitivity would circumstantially support an endocrine role for these molecules in mediating insulin resistance. On the other hand, if there were no association between adipokine/inflammatory marker concentrations and insulin sensitivity this would argue against a significant endocrine role for these molecules.

Plasma adipokine concentrations (adiponectin and resistin) and inflammatory markers (TNF$\alpha$, IL-6 and CRP) were not strongly correlated with each other at baseline or after insulin sensitization. Baseline concentrations of the inflammatory markers CRP, IL- 6 and TNF- $\alpha$ were interrelated provided that sex was not included in the analysis. These inter-relationships were not detected using the post-treatment data. TNF- $\alpha$ and IL- 6 were not correlated with parameters of insulin sensitivity and only weakly with body fatness.

These findings suggest that, although inflammatory markers may be interrelated, they are not uniformly linked with indices of insulin resistance. The inter-correlation of IL-6, TNF- $\alpha$ and CRP is consistent with our current understanding of the physiology of mild inflammation, and have also been found by some $(304,305)$, but not all $(304,306,307)$ other investigators.

The lack of consistent response of inflammatory markers after insulin sensitization suggests that insulin sensitivity per se might not be the overwhelming determinant of inflammation. We acknowledge that most $(304,305,308)$, but not all (306-309) investigators report correlations between IL-6 concentrations and insulin sensitivity, as well as with CRP (305), BMI $(305,309,310)$, TNF- $\alpha$ (304) and fatness. Plasma TNF- $\alpha$ concentrations seem to be more strongly related to anthropometric indices of obesity (304) and fat cell size (311), but not necessarily with insulin sensitivity $(304,306,308,312)$.

We found that baseline and post-treatment plasma concentrations of the adipokine resistin were not statistically related to fatness, insulin resistance or inflammation, which is consistent with recent reports (313-317). Although considered an adipokine, it appears that human adipose tissue resistin (hFIZZ3) production is primarily by macrophages and monocytes and that its actions are to stimulate lipolysis and modulate preadipocyte biology (318).

In contrast, the adipokine adiponectin was more reliably associated with insulin sensitivity markers: its baseline concentrations were correlated with insulin sensitivity parameters and after insulin sensitization using diet/exercise, this association was sustained in men. Pioglitazone treatment altered the relationship between insulin sensitivity and adiponectin, consistent with the effects of other TZDs (319).

Our findings further suggest that adiponectin, insulin sensitivity and body fat interact differently in men and women, and that the increases in adiponectin with TZDs do not predict the improvement in insulin sensitivity seen with these agents. This is consistent with the observation that TZDs, but not metformin (320) raise adiponectin levels, even in diabetic "non- 
responders' (321) and in lean, insulin sensitive subjects in the absence of insulin sensitization (322). Thiazolidinediones may affect adiponectin concentrations directly, perhaps by influencing the high-to-low molecular weight ratio (323). Mouse (323) and, more recently, human studies $(323,324)$ have suggested that not the absolute amounts, but the ratio of high molecular to low molecular weight oligomeric forms of adiponectin determine insulin sensitivity. Thiazolidinediones appear to impact the high-to-low molecular weight ratio of circulating adiponectin (323); this could explain why pioglitazone increased adiponectin concentrations much more than diet/exercise and more than could be accounted for by improvements in insulin action. Abbasi et al confirmed that rosiglitazone administration, but not caloric restriction, influenced this ratio (325). It remains to be seen whether and to what extent adiponectin directly affects insulin action in humans - this will likely require administration of adiponectin agonists or antagonists.

Baseline adiponectin and CRP concentrations were greater in women than in men despite comparable Si values (303) (5.0 \pm 0.4 (women) vs. $4.4 \pm 1.0$ (men), p=NS), which is consistent with previous reports $(326,326,327)$. We would have expected that greater adiponectin concentrations (associated with insulin sensitivity) would have been linked with lesser, not greater CRP concentrations in women. If women had less of the more biologically active high molecular weight isoforms of adiponectin (323) this could explain this finding. Unfortunately, we did not measure the high molecular weight isoform, and in any case testosterone is reported to selectively inhibit the high weight isoform (328), so women would be expected to have greater rather than lesser concentrations of this form compared with men. We are unaware of previous reports describing sex differences in the relationship between body fat and adiponectin (Figure 4.11) or reports of the association between changes in body fat and changes in adiponectin in response to treatment (Figure 4.13). Future studies will be needed to confirm our findings.

To our knowledge, ours is one of the two only studies directly comparing diet/exercise and TZDs in non-diabetic volunteers. Rosiglitazone treatment resulted in similar effects on adiponectin concentrations relative to lifestyle intervention compared with our results (319). Nonetheless, lifestyle changes have been reported to favorably affect adiponectin $(312,316,329)$, CRP $(316,321,329,330)$, IL-6 $(312,329,330)$, and TNF- $\alpha(304,312)$ in type 2 diabetic volunteers; effects on resistin are variable $(313,316)$. Thiazolidinediones effects are generally absent on IL-6 (305), variable on TNF- $\alpha$ and resistin $(312,316,331,332)$, but consistently favorable on adiponectin $(319,321,322,332)$.

The discrepancies of our findings with some other publications may result from both some limitations and strengths of our study. Our study sample was relatively small, but included carefully selected, upper body obese volunteers, who might be expected to have a narrower range of insulin sensitivity than a more diverse population. The statistical power of this relatively small sample size could be limited if the biologic variability in plasma adipokine concentrations is worse than the $16-24 \%$ variability of Si (333). Fortunately, most of the adipokines for which there is data available have less biologic variability than $\mathrm{Si}$, with the exception of IL-6 (334). It is possible that there are weaker relationships amongst adipokines or between adipokines and insulin sensitivity that we did not detect. This might also influence the statistical power of PCA to detect underlying structures in the relationships of interest. Although there is no consensus on whether absolute sample size or the ratio of subjects to items has the greatest influence hereon ${ }^{1}$, we acknowledge that a sample of 39 subjects is small and it

\footnotetext{
${ }^{1}$ (Osborne, Jason W. \& Anna B. Costello (2004). Sample size and subject to item ratio in components analysis. Practical Assessment)
} 
is unclear how much of the true factor structure can be thus identified. We nevertheless chose to use PCA as an initial screen to limit the likelihood that we would report false positive correlations (Type 2 statistical error) as a result of performing numerous regression analyses.

Moreover, not all reported studies have included more participants $(306,310,312,316,327,331,332)$ or included subjects with a greater range in Si. Some included lean $(306,308)$ and/or diabetic subjects $(306,312,321,331,335)$. The latter is especially relevant since effects of improved glycemic control may confound direct insulin sensitization effects (336-338). This phenomenon is illustrated, among others, by the absence of the sex difference in adiponectin concentrations in type 2 diabetic subjects (339).

Although our method of determining insulin sensitivity (IVGTT) is not considered as robust as the hyperinsulinemic, euglycemic clamp it is superior to HOMA analysis, fasting plasma glucose and/or insulin concentrations. Our choice of and body composition (DEXA and visceral fat by CT) is superior to methods such as bio-electric impedance analysis, DEXA only, skinfold thickness or waist circumference measurements. Thus, offsetting a relatively small number of participants, we used relatively accurate and precise measures of outcome, included equal numbers of men and women to allow us to test for sex dependent effects and employed statistical approaches (PCA) that allow discernment of underlying relationship structures. This approach reduces the chance of reporting coincidental positive correlations and can therefore be useful at detecting complex inter-relationships between adipokines and inflammatory markers, especially in large studies.

In summary, of the adipokines/inflammatory markers that we measured only adiponectin was consistently related to insulin sensitivity and body fat distribution, but changes in adiponectin were not predicted by changes in insulin sensitivity. CRP was associated with body fat and the other inflammatory markers. To the extent that correlations between insulin action and circulating hormone concentrations are proposed to mediate insulin resistance, we found no evidence that circulating resistin, TNF- $\alpha$ or IL- 6 are linked to insulin resistance. We suggest that it is important to stratify by sex when testing for relationships between adiponectin (and CRP) concentrations versus body fat and insulin sensitivity and that the use of PCA may provide a guide for further analysis of complex relationships between the numerous molecules that have become known as adipokines.

\section{Acknowledgments}

We thank Mr. W. de Brinker for his advice on our statistical analyses. 


\title{
4.5.5 Treatment of Obesity with Diet/Exercise vs. Pioglitazone Has Distinct Effects on Lipoprotein Particle Size
}

Samyah Shadid ${ }^{l}, M D$, Ralph LaForge, PhD ${ }^{2}$, James D. Otvos, PhD ${ }^{3}$ and Michael D. Jensen 4 , MD, Atherosclerosis 2006 Oct; 188(2): 370-6. Epub 2005 Nov 28

\begin{abstract}
Objective: We compared the effects of diet/exercise induce weight loss vs. pioglitazone on lipoprotein particle characteristics in non-diabetic, insulin resistant adults to determine whether the responses and potential mechanisms for improvement are similar between these two insulin sensitizing regimens.

Methods and Results: 37 obese, insulin resistant volunteers were randomized to 19 weeks of either pioglitazone or diet/exercise treatment. Insulin sensitivity (Si) by IVGTT and lipoprotein subclass particle profiles by NMR were measured. Only diet/exercise lowered TG $(-40 \pm$ $11 \mathrm{mg} / \mathrm{dl})$, total $(-29 \pm 4 \mathrm{mg} / \mathrm{dl})$ and LDL cholesterol $(-20 \pm 4 \mathrm{mg} / \mathrm{dl}$; all $\mathrm{p}<0.01)$. Both diet/exercise and pioglitazone improved $\mathrm{Si}(\mathrm{P}<0.05)$, decreased total LDL particles $(-227 \pm$ $40 \mathrm{nmol} / \mathrm{L}$ and $-149 \pm 21 \mathrm{nmol} / \mathrm{L}$, respectively, both $\mathrm{p}<0.001)$ and small LDL particles $(-242$ \pm 58 and $-226 \pm 36$, respectively, both $\mathrm{P}<0.001)$. Large LDL particle concentrations increased with both treatments. LDL particle subclasses changes correlated with changes in $\mathrm{Si}$ only in the diet/exercise group.
\end{abstract}

Conclusion: In obese, insulin resistant non-diabetic adults, pioglitazone improved LDL particle characteristics in a manner similar to diet/exercise without changing TG, diabetic control, total or LDL cholesterol. This effect could have implications for atherosclerotic risk.

\footnotetext{
${ }_{1}^{1}$ Endocrine Research Unit, Rochester, MN, USA and VieCuri Medical Center, Venlo, The Netherlands

2 Duke Lipid Disorder Preceptorship Program, Division of Endocrinology, Metabolism, and Nutrition, Duke University Medical Center, Durham, NC, USA

${ }^{3}$ LipoScience, Inc., Raleigh, NC, USA

${ }^{4}$ Endocrine Research Unit, Rochester, MN, USA
} 


\section{Introduction}

Elevated plasma LDL cholesterol (LDL-C) concentration is a well-established risk factor for coronary heart disease (CHD). The size of LDL particles is also associated with atherogenic risk: small, dense LDL particles appear to be more unfavorable than larger-size particles (340). However, the independence of the correlation of small LDL size with CHD is difficult to investigate, because a small LDL pattern rarely occurs as an isolated phenomenon. It is usually associated with an unfavorable lipid profile (high triglyceride (TG) and low HDL cholesterol (HDL-C) levels) (341) and/or the metabolic syndrome, insulin resistance and type 2 diabetes (T2DM) (342). Decreasing plasma TG concentrations and glycemic control in T2DM usually $(343,344)$, but not always $(55)$, parallels an increase in LDL particle size.

Insulin sensitizers such as pioglitazone are reported not only to improve glycemic control but to improve TG and LDL particle characteristics. Still, it is unclear whether LDL changes are part of 'automatic reshuffling' of lipid particles secondary to improved insulin sensitivity, reduced TG or blood glucose concentrations. This makes it difficult to determine to what degree LDL particle characteristics are independent contributors to the lower cardiovascular risk with successful intervention.

We found one study reporting the effects of pioglitazone, an insulin sensitizer, on lipid parameters in non-diabetic hypertensive patients (345). These investigators found that pioglitazone increased LDL size without significant TG improvement, suggesting that it is possible to dissociate these two risk factors. This study (345) did not provide data as to whether the observed LDL effects were related to insulin sensitization might be independent, specific effects of pioglitazone. Thiazolidinediones (TZDs) are known to have several unique characteristics that may be independent of their effects on glucose and fatty acid metabolism, such as decreasing liver fat content $(252,346)$ and lowering blood pressure $(347)$. It is possible that their effects on lipoproteins $(345,348)$ fall into this category.

We took advantage of samples collected from upper body obese, non-diabetic volunteers participating in a study of diet/exercise compared with pioglitazone (288) to assess the effects of these interventions on lipoprotein parameters. This eliminated possible confounding effects of improved glycemic control (as opposed to insulin sensitization) that occurs when treating T2DM, and could provide insight into whether weight loss and/or pioglitazone's effects are associated with changes in insulin sensitivity and triglyceridemia. This population was specifically selected to be insulin resistant and we made careful assessments of changes in insulin sensitivity. We measured lipoprotein characteristics using NMR spectroscopy to determine whether changes in insulin sensitivity predicted changed in LDL particles.

The results indicate that insulin sensitization without improved glycemic control increases LDL particle size, and that with pioglitazone, but not diet/exercise treatment, the changes are independent of changes in plasma TG concentrations. The distinctly different pattern of particle reshuffling between the two interventions suggests a specific pioglitazone effect on particle size that is unrelated to insulin sensitivity properties.

\section{Research Design and Methods}

\section{Subjects and Study Protocol}

Data from the 39 participants described in Chapter 4.5.2 were analyzed again, except from two men who needed niacin treatment during the course of the study based on serum TG concentrations $>500 \mathrm{mg} / \mathrm{dL}$. This would confound the interpretation of lipoprotein particle size 
changes. None of the other participants used medications with a known or suspected effect on particle size distribution. In addition to the baseline testing (blood tests and body composition measurements using CT and DEXA dual scanning), we assessed lipoprotein subclass particle profiles using NMR spectroscopy. After randomization to PIO (9 men, 10 women) or Diet/ex ( 9 men, 9 women), all tests were repeated.

Assays

Glucose, Insulin, C-Peptide and serum lipids were analyzed as previously described (Chapter 4.5.2.3). LDL Cholesterol was calculated using the Friedewald formula (349). For Lipoprotein subclasses and particle size, an NMR spectroscopic assay was performed at LipoScience, Inc. (Raleigh, NC) as previously described (350) and modified (351). In brief, the particle concentrations of lipoprotein subclasses of different size are derived from the measured amplitudes of the spectroscopically distinct lipid methyl group NMR signals they emit. The concentrations (nanomoles of particles/liter or $\mathrm{nmol} / \mathrm{L}$ ) of the following subclasses were measured: small LDL (18.0-21.2 nm), large LDL (21.2-23.0 nm), IDL (23.0-27.0 nm), large HDL (8.8$13.0 \mathrm{~nm})$, medium HDL (8.2-8.8 nm), small HDL (7.3-8.2 nm), large VLDL (>60 nm), medium VLDL (35.0-60.0 nm), and small VLDL (27.0-35.0 nm). Weighted-average lipoprotein particle sizes in nanometers $(\mathrm{nm})$ were calculated from the subclass levels and the diameters assigned to each subclass.

Table 4.5.9. Subject Characteristics, Insulin Sensitivity Parameters and Lipids

\begin{tabular}{|c|c|c|c|c|}
\hline & \multicolumn{2}{|c|}{ Diet/exercise $(n=18)$} & \multicolumn{2}{|c|}{ Pioglitazone $(n=19)$} \\
\hline & Pre & Post & Pre & Post \\
\hline \multicolumn{5}{|l|}{ Subject characteristics } \\
\hline Age (years) & $41 \pm 2$ & & $37 \pm 2$ & \\
\hline Weight (kg) & $96.9 \pm 3.4$ & $85.2 \pm 3.2 * * *$ & $98.3 \pm 2.9$ & $101.3 \pm 3.1 * * t$ \\
\hline $\operatorname{BMI}\left(\mathrm{kg} / \mathrm{m}^{2}\right)$ & $32.0 \pm 0.7$ & $27.7 \pm 0.8^{* * *}$ & $33.4 \pm 0.6$ & $34.1 \pm 0.8^{*} *$ \\
\hline Fat Free Mass (kg) & $58.4 \pm 2.5$ & $58.0 \pm 2.5$ & $57.7 \pm 2.2$ & $58.8 \pm 2.3$ \\
\hline \multicolumn{5}{|l|}{ Insulin Sensitivity } \\
\hline Fasting glucose (mg/dl) & $96 \pm 2$ & $91 \pm 2 * * *$ & $98 \pm 2$ & $93 \pm 2 * *$ \\
\hline Fasting insulin $(\mu \mathrm{U} / \mathrm{mL})$ & $8 \pm 1$ & $5 \pm 1 * * *$ & $12 \pm 1$ & $7 \pm 1 * *$ \\
\hline Fasting C-peptide (nmol/l) & $0.60 \pm 0.04$ & $0.46 \pm 0.03 * * *$ & $0.69 \pm 0.04$ & $0.51 \pm 0.03 * * *$ \\
\hline $\mathrm{Si}(\mathrm{mU} / 1)^{-1} \cdot \mathrm{min}^{-1}$ & $5.4 \pm 1.0$ & $10.7 \pm 1.9 * *$ & $4.4 \pm 0.6$ & $6.6 \pm 1.1 *$ \\
\hline \multicolumn{5}{|l|}{ Lipids } \\
\hline Triglycerides (mg/dL) & $134 \pm 16$ & $94 \pm 10^{* *}$ & $160 \pm 12$ & $145 \pm 11$ \\
\hline Total cholesterol (mg/dL) & $185 \pm 7$ & $156 \pm 6^{* * *}$ & $194 \pm 7$ & $189 \pm 7 \ddagger$ \\
\hline LDL cholesterol (mg/dL) & $119 \pm 6$ & $99 \pm 5^{* * *}$ & $128 \pm 6$ & $124 \pm 6 \ddagger$ \\
\hline HDL cholesterol (mg/dL) & $39 \pm 2$ & $38 \pm 2$ & $35 \pm 2$ & $35 \pm 2$ \\
\hline
\end{tabular}

Baseline values are not significantly different between diet/exercise and pioglitazone, except fasting plasma insulin concentrations $(\mathrm{P}=0.03 \mathrm{cf}$. between groups $)$. Si: insulin sensitivity index from the intravenous glucose tolerance test. $* \mathrm{p}<0.05 * * \mathrm{p}<0.01 * * * \mathrm{p}<0.001$ before vs. after the intervention. $\$ \mathrm{p}<0.01$ for the difference between the effect of the two interventions.

\section{Statistical Analysis}

All values are expressed as mean \pm standard error of the mean. Statistical analyses were done using SPSS 11.5. Pre- and post-intervention values were compared using paired t-tests; statistical comparisons of the two groups (pioglitazone vs. diet/exercise) and their responses to the interventions were done using $2 \times 2$ repeated measures ANOVA with factors for group 
(diet/exercise vs. pioglitazone) and time (the repeated measure - before and after intervention). All correlations mentioned are bivariate Pearson's correlations. P values $<0.05$ were considered statistically significant. To test if the relationship between the changes in LDL particle parameters and the changes in $\mathrm{Si}$ were independent of changes in plasma triglyceride concentrations we used a partial correlation analysis taking into account the change in triglycerides.

\section{Results}

Subject characteristics and baseline lipids

Anthropometric and insulin sensitivity data from 37 of the participants reported previously (288) are shown in table 4.5.9.

The diet/exercise and PIO groups were well matched for age, BMI, waist-hip ratio, insulin sensitivity parameters, and body composition. Baseline concentrations of total LDL particles (LDL-P) and small LDL particles were higher in the PIO than the diet/exercise group (table 4.5.10), but all other lipid and lipoprotein particle concentration and size parameters were similar.
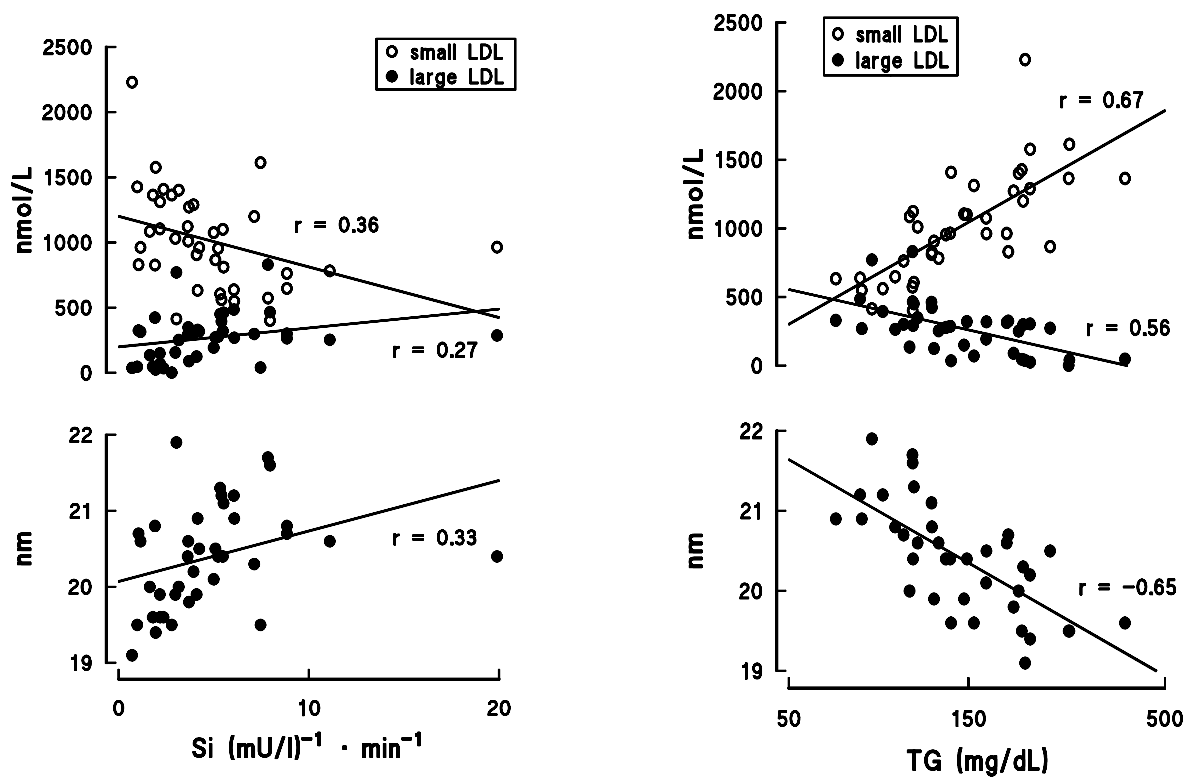

Figure 4.14 Correlations between the insulin sensitivity index (Si), triglycerides (TG) and LDL particle characteristics. Concentrations of large (open circles) and small LDL particles (closed circles) are shown in the top panels and average LDL particle size in the bottom panels. The correlations between $\mathrm{Si}$ and small LDL particle concentrations and between Si and average LDL size are statistically significant (left panel, $\mathrm{P}<0.05$ ). The correlations between plasma TG concentrations and each of the LDL characteristics shown are statistically significant (right panel, $\mathrm{P}<0.05$ ). 


\section{Lipoprotein Particle Size Relationships}

The baseline insulin sensitivity index ( $\mathrm{Si}$ ) correlated significantly with small LDL-P concentrations $(\mathrm{r}=-0.36, \mathrm{p}=0.02$; figure 4.14 , upper panel). The correlations between $\mathrm{Si}$ and LDL size $(r=0.33, p=0.04$; figure 4.10 , lower panel) and large LDL-P-concentrations $(r=0.27$, $\mathrm{p}=0.09$; figure 4.14 , upper panel) were less strong, and those between $\mathrm{Si}$ and HDL size $(\mathrm{r}=0.27, \mathrm{p}=0.10)$ and VLDL size $(\mathrm{r}=-0.26, \mathrm{p}=0.12)$ were weaker yet.

Fasting plasma TG concentrations were highly correlated with concentrations of small LDL-P $(\mathrm{p}<0.001$, figure 4.14, upper panel) and inversely with LDL size $(\mathrm{P}<0.001$, figure 4.14 , lower panel) and large LDL-P (figure 4.14, upper panel; $\mathrm{P}<0.001$ ).

\section{Response To Interventions}

Changes in insulin sensitivity parameters were similar between the diet/exercise and pioglitazone treated groups (table 4.5.9); the increase in Si was not significantly different $(p=0.12)$ between the groups

Diet/exercise decreased serum triglycerides, total and LDL- cholesterol, but not HDL cholesterol concentrations (table 4.5.9). Average LDL and HDL particle sizes increased (table 4.5.10), resulting from increased concentrations of the large, and reduced concentrations of the small particle subclasses (table 4.5.10). Total VLDL particle concentration was significantly reduced, but VLDL particle size was unchanged since each VLDL subclass was reduced to a comparable extent.

Table 4.5.10. Lipoprotein particle sizes and concentrations

\begin{tabular}{lll|ll}
\hline & \multicolumn{2}{c}{ Diet/exercise } & \multicolumn{2}{c}{ Pioglitazone } \\
\cline { 2 - 5 } & before & after & before & after \\
\hline Sizes $(\boldsymbol{n m})$ & & & \\
VLDL & $49.7 \pm 1.4$ & $49.5 \pm 1.4$ & $50.6 \pm 1.2$ & $48.4 \pm 1.0^{* * *}$ \\
LDL & $20.6 \pm 0.2$ & $20.9 \pm 0.1^{* *}$ & $20.3 \pm 0.2$ & $20.7 \pm 0.2^{* *}$ \\
HDL & $8.8 \pm 0.1$ & $9.0 \pm 0.1^{* * *}$ & $8.8 \pm 0.1$ & $8.8 \pm 0.1$ \\
Concentrations & & & & \\
Total VLDL $(\mathbf{n m o l} / \mathbf{L})$ & $82.3 \pm 6.8$ & $58.0 \pm 6.7^{* * *}$ & $101.8 \pm 8.9$ & $99.0 \pm 9.1$ \\
Large VLDL $(\mathrm{nmol} / \mathrm{L})$ & $3.4 \pm 1.0$ & $1.4 \pm 0.7^{* * *}$ & $4.5 \pm 0.7$ & $3.9 \pm 0.8$ \\
Medium VLDL $(\mathrm{nmol} / \mathrm{L})$ & $36.1 \pm 4.3$ & $24.2 \pm 4.0^{* *}$ & $48.4 \pm 5.4$ & $43.8 \pm 5.7$ \\
Small VLDL $(\mathrm{nmol} / \mathrm{L})$ & $42.9 \pm 3.5$ & $32.4 \pm 3.1 * * *$ & $48.9 \pm 4.4$ & $51.3 \pm 4.9$ \\
Total LDL $(\mathbf{n m o l} / \mathrm{L})$ & $1216 \pm 55$ & $988 \pm 46^{* * *}$ & $1420 \dagger \pm 74$ & $1270 \pm 81^{* * *}$ \\
IDL $(\mathrm{nmol} / \mathrm{L})$ & $26.4 \pm 5.8$ & $6.6 \pm 2.5^{* *}$ & $44.5 \pm 8.2$ & $42.4 \pm 7.4$ \\
Large LDL $(\mathrm{nmol} / \mathrm{L})$ & $300.8 \pm 41.0$ & $335.3 \pm 30.2$ & $255.9 \pm 45.9$ & $334.9 \pm 45.1 * *$ \\
Small LDL $(\mathrm{nmol} / \mathrm{L})$ & $888.5 \pm 74.8$ & $646.5 \pm 55.0^{* *}$ & $1119.4 \dagger \pm 94.6$ & $893.1 \pm 102.0^{* * *}$ \\
Total HDL $(\mu \mathrm{mol} / \mathrm{L})$ & $28.6 \pm 0.9$ & $27.3 \pm 0.8^{*}$ & $27.3 \pm 1.0$ & $26.7 \pm 1.0$ \\
Large HDL $(\mu \mathrm{mol} / \mathrm{L})$ & $4.8 \pm 0.7$ & $6.1 \pm 0.7^{* *}$ & $4.1 \pm 0.6$ & $4.7 \pm 0.6^{* *}$ \\
Medium HDL $(\mu \mathrm{mol} / \mathrm{L})$ & $3.7 \pm 0.6$ & $3.5 \pm 0.6$ & $4.1 \pm 0.8$ & $5.6 \pm 1.0^{*}$ \\
Small HDL $(\mu \mathrm{mol} / \mathrm{L})$ & $20.1 \pm 0.7$ & $17.6 \pm 0.8^{* * *}$ & $19.2 \pm 0.8$ & $16.4 \pm 0.9 * *$ \\
\hline
\end{tabular}

Only baseline LDL particle number differed between diet/exercise and pioglitazone groups $(\uparrow \mathrm{p}<0.05){ }^{*} \mathrm{p}<$ $0.05, * * \mathrm{p}<0.01, * * * \mathrm{p}<0.001$ before vs. after the intervention.

Pioglitazone treatment changed neither TG nor total LDL cholesterol concentrations significantly (table 4.5.9), but significantly increased average LDL particle size: this resulted from a substantial decrease in small LDL-P and an accompanying increase in large LDL-P concentrations. Pioglitazone treatment did not affect HDL size, but decreased VLDL size: large and 
medium VLDL subclass levels decreased and small VLDL increased, neither of which however statistically significantly.

We examined the relationship between the changes in LDL subclass concentrations and the changes $\mathrm{Si}$ and in plasma $\mathrm{TG}$ concentrations in response to the interventions. After diet/exercise, changes in $\mathrm{Si}$ correlated with changes in both large and small LDL-P (figure 4.16). These correlations were sustained after accounting for the change in plasma TG concentrations ( $\mathrm{r}=0.48, \mathrm{p}=0.05$ and $\mathrm{r}=-0.49, \mathrm{p}=0.03$ respectively). This association was not seen after pioglitazone treatment $(\mathrm{r}=0.00$ and $0.03, \mathrm{p}=\mathrm{NS}$ respectively, figure 4.16$)$, whether or not the change in TG was factored in: $r=0.05$ and $r=-0.06, p=N S$, respectively for the correlation between the change in Si and changes in large and small LDL-P. Changes in LDL subclasses did not correlate with the changes in plasma TG concentrations for either group (data not shown).

As might be expected, the change in Si significantly correlated with the change in average LDL particle size in the diet/exercise group (figure 4.16; $r=0.56, P=0.02 ; r=0.61, p=0.009$ when corrected for the change in TG); this was not the case in the pioglitazone group $(\mathrm{r}=$ 0.04 for the simple correlation, $r=0.03$ after correction for the change in plasma TG concentrations, both $\mathrm{p}=\mathrm{NS}$ ).

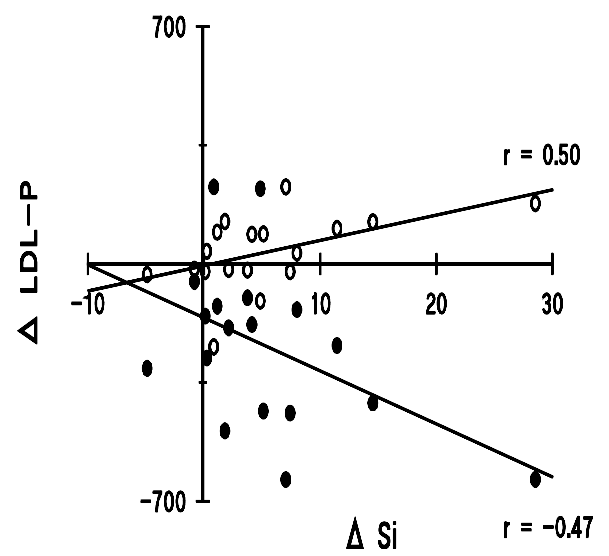

Figure 4.15 Correlation between the change in Si and the change in $L D L$ particle numbers after diet/exercise.

Filled circles represent changes in small LDL-particle concentration and open circles represent changes in large LDL-

$P$. The correlations between postintervention changes in $\mathrm{Si}$ and both small and large LDL-P are significant $(\mathrm{P}<$ $0.05)$.

\section{Discussion}

The primary goal of this study was to compare the effects of pioglitazone and diet/exercise on body fat, glucose and lipid metabolism (288). The opportunity to evaluate quantitative measures of VLDL, LDL, and HDL subclasses using NMR spectroscopy allowed us to contrast the impact of these two insulin-sensitizing regimens on lipoprotein atherogenic risk factors. The lack of TG lowering after pioglitazone treatment further allowed us to study a possible uncoupling of LDL particle size changes from the usual lipoprotein improvements. The diet/exercise group allowed us to test the association of LDL changes with changes in insulin sensitivity and, by contrast, determine whether a similar association could be detected in the pioglitazone treated group. The effect of diabetic control was eliminated by studying non- 
diabetic, insulin resistant volunteers. Although only diet/exercise reduced total cholesterol, TG, and absolute LDL concentrations, both interventions increased number of large LDL particles and decreased the number of small LDL particles. This resulted in a significant overall reduction in total LDL particle concentration following both treatments. Thus, these two approaches to treating the atherogenic risk state of insulin resistant obesity have similar LDL effects yet distinctly different patterns of achieving that response.

The pattern of the VLDL and IDL particle changes differed in some aspects between the two treatment approaches in that improvements were generally greater following diet/exercise than after pioglitazone. If changes in insulin sensitivity primarily mediate the changes in plasma lipoprotein particles we would have expected the same changes in both groups because the improvement in insulin sensitivity, as measured by $\mathrm{Si}$, was similar between
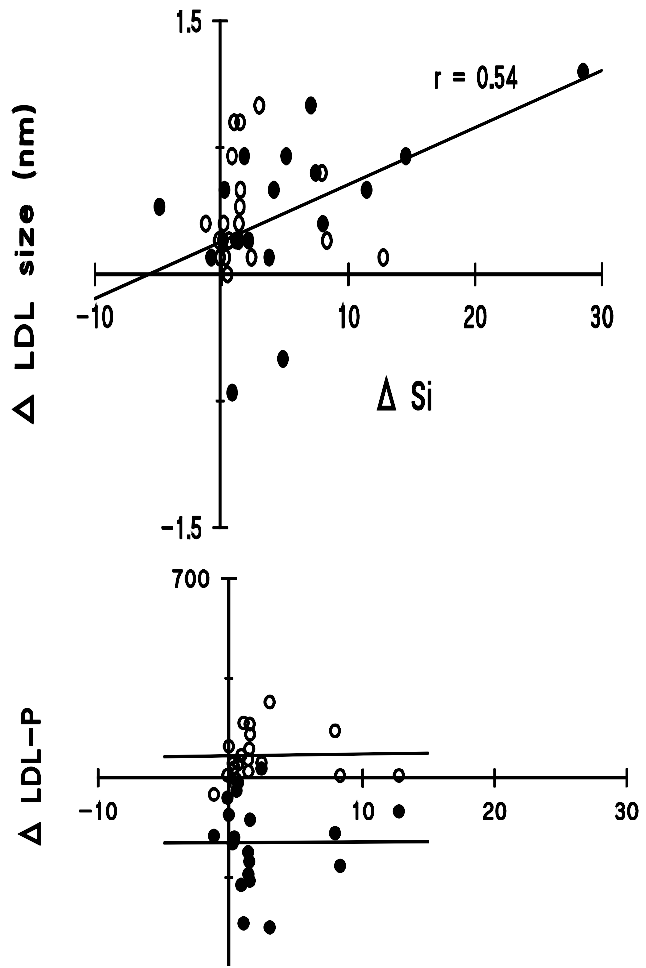

Figure 4.16 Correlation between the change in Si and the change in average LDL particle size. Filled circles represent values from diet/exercise-treated subjects and open circles represent values from those treated with pioglitazone. The correlation between post-intervention changes in $\mathrm{Si}$ and average LDL size is significant $(\mathrm{P}<0.05)$ only for diet/exercise. diet/exercise and pioglitazone. The fact that the patterns were different suggests that the changes in LDL subclass distribution are not merely the effects of insulin sensitization per se, at least for the pioglitazone intervention. This suggestion is strengthened by the observation that the correlation between LDL subclasses and $\mathrm{Si}$ at baseline was sustained after diet/exercise, but was lost after pioglitazone treatment.

With pioglitazone treatment the increase in LDL particle size was highly statistically significant, but no significant

changes in plasma TG or VLDL subclasses were observed. We speculate that the LDL change with pioglitazone is not due to insulin sensitization per se, nor to "reshuffling" of lipoprotein particle composition that usually accompanies changes in VLDL concentrations (341).

NMR spectroscopy is a relatively new lipoprotein measurement technique and few comparisons exist with other subfractionation methods. We were therefore gratified to find that the changes in LDL characteristics in our volunteers treated with pioglitazone are the same as those reported by Winkler et al (345), who used the more labor-intensive technique of density gradient ultracentrifugation to measure lipoprotein responses to pioglitazone in hypertensive subjects. Likewise, the changes seen after diet/exercise treatment in our volunteers are consistent with those shown by NMR following diet (352) exercise training (353) and reported by investigators using different methods to determine LDL particle size (345). Thus, we believe that the changes in lipoprotein particle number and size we found using NMR spectroscopy are reliable indications of the effects of these two interventions. 
We were not able to address the mechanism for pioglitazone-induced changes in LDL particle size. Possibilities include changed activities or mass of lipoprotein lipase, which is known to be influenced by pioglitazone, and/or hepatic lipase $(354,355)$. Hepatic lipase is, in part, responsible for the interconversion between large and small LDL as well as large and small HDL particles. The fact that both large LDL and HDL subclass levels were increased by pioglitazone, while small LDL and HDL levels were reduced, would be consistent with an effect on hepatic lipase. In support of this potential mechanism, the induction of insulin resistance in animal models parallels an increase in hepatic lipase mRNA and plasma activity that can be partially normalized by rosiglitazone treatment (356).

Another possible explanation relates to LDL clearance, i.e. greater removal of potentially atherogenic particles rather than their reduced production, e.g. via CD 36. This fatty acid transport protein also binds modified LDL particles and its expression is reported to increase by pioglitazone (357).

The relevance of our findings to clinical practice relates to the fact that quantitative measures of LDL cholesterol might not reflect the full 'lipid burden'. In our study, LDL-C decreased $22 \%$ after diet/exercise and only $2 \%$ after pioglitazone. LDL-P, however, decreased by 19 and $11 \%$ after the respective interventions. Thus, a potentially clinically relevant change in LDL burden brought about by pioglitazone would not have been detected by conventional lipid analysis only. It may, therefore, be important for clinicians to appreciate that (changes in) absolute LDL-C concentrations may not always reflect atherosclerotic risk (358), even in subjects with only slightly elevated TG concentrations (e.g, in the 100-200 mg/dL range). This may be especially true for pioglitazone treated patients. Nevertheless, it has yet to be established to what extent the combination of LDL particle lowering of this magnitude and the changes in subclass particle distribution contribute to predicting or lowering the atherosclerotic risk.

Several limitations to this study must be acknowledged. The statistical significance of the diet/exercise results were influenced by one volunteer who lost $15 \mathrm{~kg}$ and had the greatest improvement in insulin sensitivity; the correlation between the changes in Si and LDL size was not statistically significant if these data points were excluded from the analysis. Because all of the data from this volunteer were internally consistent, however, we have no reason to suspect that its inclusion in our analysis has led to inaccurate conclusions. None of the participants in the pioglitazone group had that degree of improvement in $\mathrm{Si}$; it is possible that if such an improvement occurred with pioglitazone we could have detected an association between Si and LDL size. Another limitation is that our sample size was relatively small given the inherent biological variability in some of the parameters, making direct comparisons between diet/exercise and pioglitazone difficult. However, our goal was not necessarily to perform a quantitative head-to-head comparison between the pioglitazone and diet/exercise intervention, but to use diet/exercise as a control treatment to determine whether the lipid effects of pioglitazone are perfectly mimicked by another methods of improving insulin sensitivity. Given the relatively small sample size and post hoc nature of the research study question our results should be confirmed by future, prospective studies.

In conclusion, the shift of LDL particle subclass distribution towards larger particles after insulin sensitization is independent of improvements in diabetic control. With diet/exercise, but not pioglitazone treatment, this shift correlates with the improved insulin sensitivity; after pioglitazone, it is also independent of changes in triglyceride concentrations. Since this therefore does not relate to a non-specific effect of insulin sensitization it may be a more specific effect of TZDs. 


\title{
4.5.6 Effects Of Diet And Exercise Versus Pioglitazone On Metabolic Health And Fat Distribution In Upper Body Obesity
}

\author{
Samyah Shadid and Michael D. Jensen. Diabetes Care 26:3148-3152, 2003 \\ Endocrine Research Unit, Mayo Clinic, Rochester, Minnesota, USA
}

\begin{abstract}
Background: Insulin resistance is associated with visceral adiposity and interventions that reduce this depot, e.g. diet and exercise, improve insulin resistance. Thiazolidinediones (TZD) also improve insulin action but paradoxically increase total fat mass, perhaps through remodeling (recruitment of smaller fat cells) and redistribution of adipose tissue. We assessed the effects of pioglitazone vs. diet/exercise on fat distribution and the relationship between fat distribution and insulin sensitivity in upper body obesity (UBO).

Methods: 39 UBO insulin resistant, non-diabetic men and pre-menopausal women were randomly assigned to receive either pioglitazone (PIO) $30 \mathrm{mg} /$ day or a diet and exercise program for 20 weeks. Before and after the intervention, insulin sensitivity, body composition, body fat distribution (WHR, CT abdomen and DEXA), and abdominal and femoral fat cell size were assessed.

Results: Diet/exercise resulted in an $11.8 \pm 1.1 \mathrm{~kg}$ weight loss. Both diet/exercise and PIO improved insulin sensitivity, but only the former was associated with loss of intra-abdominal fat. PIO increased total body fat, which preferentially accumulated in the lower body depot in both men and women. Waist-hip ratios decreased in both groups. Abdominal fat cell size decreased $(p=0.06)$ after diet/exercise. No statistically significant changes in fat cell size were observed in PIO treated volunteers.

Conclusion: In non-diabetic UBO, increasing insulin sensitivity via diet and exercise accompanies reductions in visceral fat. PIO treatment also improves insulin sensitivity and lowers WHR, but this is due to a selective increase in lower body fat. This confirms a site-specific responsiveness of adipose tissue to TZD and suggests that improvements in insulin sensitivity by PIO are achieved independent of changes in intra-abdominal fat.
\end{abstract}




\section{Introduction}

Body fat distribution is an important variable in the relationship between overweight and insulin resistance. Intra-abdominal or visceral fat accumulation is more strongly associated with insulin resistance, and insulin resistance can be improved by decreasing this fat depot via diet, exercise or surgery $(10,359)$. Thiazolidinediones (TZD) are also known to improve insulin sensitivity despite paradoxically increasing total fat mass $(260,360,361)$. It has been suggested that redistribution of body fat may contribute to their insulin sensitizing qualities. Indeed, regional variability in TZD responsiveness has been demonstrated: pre-adipocytes from subcutaneous fat differentiate more in response to TZD in vitro than visceral adipose tissue (240). If the same phenomenon occurs in vivo one would expect selective adipocyte proliferation and thus body fat redistribution.

However, studies of animals and diabetic humans have reported increasing, decreasing and unchanged visceral and subcutaneous fat depots after TZD administration despite improved insulin sensitivity $(260,360-363)$. This suggests that TZD effects on visceral adiposity, if present in vivo, might not contribute to their insulin sensitization.

We assessed the effects of pioglitazone on fat distribution, fat cell size as well as the relationship between fat distribution and insulin sensitivity in UBO, a known insulin resistant state. Comparison of the effects of PIO with diet and exercise, a standard intervention to improve insulin sensitivity, was performed to place the results in context.

\section{Methods}

\section{Subjects and Study Protocol}

Results of the 39 volunteers who completed both studies (Chapter 4.5.2) were analyzed. Baseline blood draws (complete blood count, chemistry panel, lipid profile), an intravenous glucose tolerance test, body composition measurements, fitness testing and adipose tissue biopsies were assessed as described in Chapter 4.5.2, before and after randomization to $30 \mathrm{mg}$ PIO daily or a personalized, non-supervised diet/ex program.

Body composition was assessed through analysis of CT images combined with data from DEXA (60) as previously described. The following compartmental fat volumes were calculated: total body fat, lower body fat, upper body non-visceral fat and visceral (intraabdominal) fat (Figure 4.6). Procedures for femoral and abdominal subcutaneous fat biopsies and fat cell sizing, as well as the assays used are also described in paragraph 4.5.2.3. Cell volumes were calculated using the Goldrick formula (364). Adipocellular lipid content was calculated as fat cell volume $\times 0.95$.

\section{Statistical Analysis}

Values are expressed as mean \pm standard error of the mean. Statistical comparisons of the two groups (PIO vs. diet/exercise) and the responses of the groups to the interventions were done using repeated measures ANOVA followed by t-tests (paired or non-paired) if needed. P values $<0.05$ were considered statistically significant.

\section{Results}

Subject characteristics. Nineteen volunteers (10 men, 9 women) completed the diet/exercise intervention and 20 volunteers (10 men and 10 women) completed the PIO intervention. The 
two groups were well matched for age, BMI, waist-hip ratio (WHR), insulin sensitivity parameters and body composition (see tables 4.5.1-4.5.2). The pre-intervention fasting plasma insulin concentrations were greater $(\mathrm{P}<0.05)$ in the PIO than in the diet/exercise group.

Relationship between body composition and metabolic variables. At baseline, fasting plasma insulin concentrations correlated with weight $(\mathrm{r}=0.37, \mathrm{P}<0.05)$, waist circumference $(\mathrm{r}=$ $0.35, \mathrm{P}<0.05)$, and WHR $(\mathrm{r}=0.37, \mathrm{P}<0.05)$. C-peptide was significantly correlated with these parameters as well as with BMI $(\mathrm{r}=0.33, \mathrm{P}<0.05)$ and visceral fat area $(\mathrm{r}=0.33, \mathrm{P}<$ $0.05)$. At baseline $\mathrm{Si}$ only correlated positively with HDL, and negatively with WHR $(\mathrm{r}=$ $0.36, \mathrm{P}<0.05)$ and negatively with total abdominal fat area by $\mathrm{CT}(\mathrm{r}=-0.36, \mathrm{P}<0.05)$.

Body composition changes in response to intervention. The diet/exercise program induced a weight loss of $11.8 \pm 1.1 \mathrm{~kg}(9.5 \pm 1.0$ for women, $13.9 \pm 1.5 \mathrm{~kg}$ for men) by loss of fat, not FFM. Because adipose tissue mass is $85 \%$ lipid and $15 \%$ water (365), and because DEXA measurement of FFM includes this adipose tissue water, the loss of adipose without loss of FFM suggests favorable changes in muscle mass.

Proportionately more abdominal than femoral, and proportionately more visceral than subcutaneous abdominal fat was lost as evidenced by the changes in WHR, the ratio of visceral fat to subcutaneous abdominal fat area (v/s ratio) and the various fat compartments measured by DEXA (Table 4.5.11). Fat cell lipid content decreased in the abdominal $(\mathrm{p}=0.06)$, but not the femoral depot $(\mathrm{p}=0.33)$ with diet/exercise.

In the PIO group, the average weight gain of $2.7 \pm 0.7 \mathrm{~kg}$ was attributed to an increase in fat $(1.3 \mathrm{~kg})$, predominantly in the leg depot, and to increased FFM $(1.1 \mathrm{~kg}, \mathrm{p}=0.07)$. There was no change in abdominal fat compartments (visceral or upper body non-visceral). Consistent with the DEXA and CT data, WHR decreased due to increased hip, but not waist circumference. The average adipocyte lipid content after the PIO treatment was less in both the femoral and abdominal sites but the difference from baseline were not statistically significant (decrease by $0.09 \mu \mathrm{g}$ lipid/cell in femoral, $\mathrm{p}=0.15$; decrease by $0.06 \mu \mathrm{g}$ lipid/cell in abdominal, $\mathrm{p}=0.23)$.

Metabolic response to intervention. The increase in insulin sensitivity index ( $\mathrm{Si}$ ) was greater in the diet/exercise than in the PIO group, although the difference between the two treatments was not statistically significant $(\mathrm{p}=0.15)$. Decreases in fasting plasma glucose and C-peptide concentrations were similar in both groups. The changes in serum lipid concentrations were more marked in the diet/exercise group; the only significant between-group difference, however, was for serum total cholesterol (Table 4.5.2). The greater decrease in insulin in the pioglitazone group is confounded by higher baseline concentrations. In the diet/exercise group blood pressure decreased from $128 \pm 4 / 82 \pm 2$ to $122 \pm 3 / 76 \pm 2(\mathrm{P}<0.0001$ for systolic and $\mathrm{P}$ $=0.07$ for diastolic) and in the PIO group blood pressure decreased from $129 \pm 4 / 80 \pm 3$ to $127 \pm 3 / 75 \pm 2(\mathrm{P}<0.05$ for diastolic only $)$.

Relationship between change in body composition and change in metabolic variables. Changes in Si were not significantly correlated with changes in body composition in either group (results not shown). In addition, changes in glucose, C-peptide, or Si did not correlate with changes in femoral or abdominal fat cell size in either group. 
Table 4.5.11 Effects of diet/exercise and pioglitazone on fat distribution and fat cell size.

\begin{tabular}{|c|c|c|c|c|}
\hline \multicolumn{2}{|c|}{ Diet/exercise } & \multicolumn{2}{|c|}{ Pioglitazone } & \multirow[b]{2}{*}{$\mathrm{p}$ delta } \\
\hline CT-abdomen & post & pre & post & \\
\hline SC fat $\left(\mathrm{cm}^{2}\right) 259 \pm 15$ & $196 \pm 18 * * *$ & $317 \S \pm 18$ & $328 \pm 23$ & 0.04 \\
\hline visceral $\left(\mathrm{cm}^{2}\right) 203 \pm 29$ & $123 \pm 22 * * *$ & $154 \pm 15$ & $154 \pm 17$ & 0.0001 \\
\hline visceral $/ S C 0.80 \pm 0.11$ & $0.66 \pm 0.11 * * *$ & $0.50 \S \pm 0.05$ & $0.48 \pm 0.05$ & 0.007 \\
\hline \multicolumn{5}{|l|}{ DEXA fat (kg) } \\
\hline total body $35.8 \pm 1.5$ & $26.5 \pm 1.9 * * *$ & $38.0 \pm 1.5$ & $39.3 \pm 1.7^{* *}$ & 0.0001 \\
\hline $\operatorname{leg} 11.7 \pm 0.8$ & $8.7 \pm 0.8 * * *$ & $13.8 \pm 0.8$ & $14.8 \pm 0.9^{* * *}$ & 0.0001 \\
\hline \multicolumn{5}{|l|}{ CT+DEXA fat $(\mathrm{kg})$} \\
\hline UBNV18.3 \pm 0.7 & $14.5 \pm 1.0 * * *$ & $19.8 \pm 0.9$ & $20.2 \pm 0.8$ & 0.0001 \\
\hline visceral5.8 \pm 0.6 & $3.3 \pm 0.5 * * *$ & $4.4 \pm 0.4$ & $4.3 \pm 0.4$ & 0.0001 \\
\hline \multicolumn{5}{|l|}{$\begin{array}{l}\text { Fat cell size } \\
(\mu \mathrm{g} \text { lipid/cell) }\end{array}$} \\
\hline abdominal $0.84 \pm 0.19$ & $0.68 \pm 0.16$ & $0.81 \pm 0.18$ & $0.75 \pm 0.17$ & 0.52 \\
\hline femoral $0.85 \pm 0.19$ & $0.79 \pm 0.18$ & $0.85 \pm 0.19$ & $0.76 \pm 0.17$ & 0.60 \\
\hline
\end{tabular}

Baseline values are not significantly different between diet/exercise and pioglitazone except in subcutaneous fat and therefore the visceral/SC ratio $\S \mathrm{P}<0.05$ diet/exercise vs pioglitazone at baseline; $* \mathrm{p}<0.05 * * \mathrm{p}<0.01 * * *$ $\mathrm{p}<0.001$ before vs. after the intervention; $\mathrm{p}$ delta signifies the difference between the effect of the two interventions. CT: computed tomography; DEXA: dual energy X-ray absorptiometry; SC: subcutaneous; UBNV: upper body non-visceral.

\section{Discussion}

We compared the effects of two insulin-sensitizing regimens, pioglitazone vs. diet/exercise, on body composition, body fat distribution and insulin sensitivity. Non-diabetic, UBO adults were studied because of the high prevalence of insulin resistance in this population. The anticipated improvement in Si occurred with each treatment, and the change in body fat compartments in response to diet/exercise was consistent with previous reports.

We unexpectedly found that PIO resulted in the preferential accumulation of lower body fat rather than loss of visceral fat. Thus, both diet/exercise and PIO resulted in a reduced WHR but the mechanism was quite different. The shift towards a lower body fat distribution by PIO via gain of leg fat, not loss of visceral fat, is consistent with adipose depot specific responses, but not of the type previously reported.

The lack of change in intra-abdominal adipose tissue area with PIO is consistent with some, but not all, previous findings. Four reports described no change (261,360-362) and 3 a decrease $(260,281,362)$. Most $(261,281,360-362)$, but not all (360) reported a decrease in the visceral to subcutaneous abdominal fat ratio. However, the investigators reporting reductions in visceral fat combined TZDs with energy-restricted diets $(281,362)$ or other medication (260), which may have modulated the TZD effects. These investigators studied the effects of TZDs in Type 2 diabetic adults, a different study population from our insulin resistant, nondiabetic volunteers. Although it is possible that the response to TZDs is different between diabetic and non-diabetic humans we note that a trend towards decreasing WHR despite an increasing waist diameter was noted in adults with type 2 diabetes (260), suggesting that our finding is not entirely unique to non-diabetic volunteers.

PIO increased leg fat without influencing upper body fat mass. The trend towards smaller femoral fat cell size in the PIO group in the face of increased leg fat mass suggests adipocyte proliferation rather than hypertrophy was responsible for the leg fat gain. This would be consistent with the PPAR- $\gamma$ agonist effects of PIO on preadipocytes. If the improvements in insulin sensitivity we observed are related to changes in adipose tissue metabolism, these data 
suggest an independent role for the relative amount of lower body fat. Alternatively, PIO may improve Si and increase lower body fat independently.

A number of investigators have examined the site-specific actions of TZD on adipose tissue remodeling. In vitro responsiveness of abdominal subcutaneous, but not omental human preadipocytes to TZD has been reported (240). In vivo, ovarian AT was more sensitive to TZD than retroperitoneal or subcutaneous abdominal fat Zucker rats (363). Although not specifically supportive of our observations regarding leg fat, both findings suggest a regional difference in TZD sensitivity.

As expected, Si correlated with WHR and total abdominal fat at baseline. This was expected given the known association between insulin resistance and visceral fat accumulation (366). The relative weakness of the correlation coefficients between $\mathrm{Si}$ and anthropometric/body composition parameters in our population are likely due to the selection of the participants: the narrow range of $\mathrm{Si}$ and body fat/fat distribution variables reduced the strength of the associations. Given the weak correlation coefficients between $\mathrm{Si}$ and body composition at baseline it is not surprising that changes in Si were not significantly correlated with changes in the parameters we assessed with regards to adipose tissue mass, distribution or cellularity. The relatively small inter-individual variation in fat and visceral fat loss in the diet/exercise group, combined with inherent individual differences in the Si response to weight loss, may limit the ability to detect a correlation between fat loss and improvement in Si. Alternatively, improvements in Si from 5 months of exercise and energy restriction may be independent of and greater than the effects of regional fat loss, such that the underlying relationship is undetectable. It is also possible that the precision of measurement of insulin action with the IVGTT is insufficient for detecting a relationship, despite the relatively accurate measures of fatness.

In conclusion, weight loss via diet/exercise and PIO improve insulin sensitivity and shift adipose tissue towards a lower body fat distribution in upper body obese, non-diabetic adults. PIO selectively increased lower body fat, apparently via adipocyte proliferation whereas preferential loss of visceral/upper body subcutaneous fat occurred with diet/exercise. Understanding the depot specific action of TZD may help define the insulin sensitizing properties of this class of compounds. 


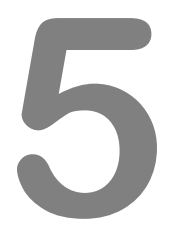

\section{Summaries and Future Perspectives}


5.1 Summary and Future Perspectives

5.2 Nederlandse Samenvatting (Summary in Dutch) 


\subsection{Summary and Future Perspectives}

\section{General considerations}

The exponential rise in the prevalence of obesity and its complications increasingly challenges world health and health care load. Understanding the pathogenesis of obesityassociated complications and possible intervention options is needed, if large scale, long-term treatment is to be achieved.

This thesis has focused on the pathophysiology of obesity-associated changes in fatty acid metabolism and insulin sensitivity, and the reversal hereof. Weight loss through diet and exercise (Diet/ex) has been extensively studied and may be viewed as the 'gold standard' of insulin sensitization. Pharmacological insulin sensitization using thiazolidinediones (TZDs) has also proven to be effective, but is poorly understood in terms of the exact mechanisms of action. We therefore studied various aspects of adipose tissue physiology, insulin sensitivity and fuel trafficking in lean and obese volunteers, before and after insulin sensitization using Diet/ex or the TZD Pioglitazone (PIO).

After a global overview of the magnitude and effects of the obesity epidemic, Chapter 1 provides an outline of the thesis. In Chapter 2, a synopsis of current knowledge on fatty acid and adipose tissue metabolism is given, followed by new insights herein based on original experiments.

In Chapters 3 and 4 we describe a series of original experiments contrasting metabolic aspects of the thiazolidinedione pioglitazone (PIO) to Diet/ex treatment. This allowed us to study specific PIO effects and to distinguish them from non-specific effects of insulin sensitization. We chose to study non-diabetic insulin resistant adults rather than type 2 diabetic volunteers, in order to additionally differentiate insulin-sensitizing effects from those of improved glycemic control on insulin action.

\section{A novel pathway of fat uptake independent of VLDL was discovered. This is regionally variable and gender-specific and might contribute to body fat distribution.}

Despite the importance of fat distribution to metabolic health, little research exists on the physiology of inter-individual and gender differences herein. Chapter 2.2 reports the results an original study of the existence of direct free fatty acid (FFA) uptake into adipose tissue, independently of VLDL, and the possible role hereof in body fat distribution.

After developing and validating the required methods, we demonstrated that FFAs are incorporated directly into intra-adipocyte TG to a significant extent. Tracing an intravenous ${ }^{14} \mathrm{C}$ FFA bolus in 12 lean men and 15 lean women into abdominal and femoral subcutaneous fat revealed marked sex differences herein: direct FFA uptake was $\sim 70 \%$ more efficient in women than men, and systemic FFA uptake in subcutaneous fat in women was double that in 
men $(-8.2 \pm 0.6 \%$ vs. $4.0 \pm 0.5 \%(\mathrm{P}<0.0001))$. In men, but not in women, abdominal subcutaneous fat took up FFA more avidly than femoral fat.

Similarly, in obese women $(n=12)$ and men $(n=10)$, we demonstrated that, even after total recycling of tracer from FFA back into adipocytes, this sex-specific pattern was maintained, including a greater efficiency of FFA storage and a preferential femoral uptake in women compared to men. This was done using 6-hour continuous ${ }^{14} \mathrm{C}$-palmitate infusions, again followed by fat biopsies.

Although the latter observations result from both direct and VLDL-incorporated FFA uptake, the contribution of the latter was likely minor, considering the much lower turnover of VLDLTG compared to FFA (19). However, irrespective of their origin, the pattern of uptake of (re)circulating FFAs is consistent with the lesser subcutaneous body fat mass and the upper body fat distribution in men. Gene (53) expression of fatty acid transporters (greater in abdominal than femoral fat in men $(\mathrm{P}<0.05)$, but not in women $(\mathrm{P}=0.80))$ pointed towards the same.

We conclude that sex- and site-specific uptake of circulating FFA into subcutaneous fat is a novel FFA disposal pathway that may play a role in the development or maintenance of body fat distribution. Regional variations in facilitated FA transport may contribute to this process.

In the fasted state, there is a net outward flow of FFA from AT. To date, fat uptake in AT is thought to occur via VLDL or chylomicrons under the influence of LPL only. This is process is particularly well controlled by key hormones such as insulin, catecholamines and cortisol (Chapter3.2).

Our newly found direct FFA uptake, independently of VLDL/LPL, implies not only the bypassing of several regulatory steps of adipocyte dynamics as presently known, but would also require FFAs to "swim against the current". If left to passive diffusion (the so-called "flipflop" mechanism), this process would therefore be highly unlikely to occur; facilitated transport would be required. This is compatible with the differential membrane protein expression pointing into the same direction as the tracer findings.

The function of this counter-intuitive direct FA uptake remains puzzling. It could serve as a rapid, effective buffer for unexpected, unregulated or excess/spill-over FA challenges, which would otherwise be presented to other organs in an unregulated manner. In its absence, this might perhaps lead to ectopic fat deposition or disturbances of glucose/FFA interaction and partitioning.

One would expect these fat loads to occur postprandially more than in the post-absorptive state. Indeed, FA transporter protein expression is controlled, among others, by insulin $(367,368)$; insulin clamps and/or postprandial studies could further increase our insight into the regulation and the magnitude of this process. Since most people are in a fed state the majority of the day this could be particularly relevant.

In our studies, FATP4 seemed to be the transporter protein involved above all. It should, however, be noted that mRNA expression, however indicative, might not necessarily reflect the actual protein content and/or function in the cells or the cell membranes. We are currently determining the concentrations of the actual proteins in the adipose tissue from the above-mentioned studies (Western blotting). Another possibility for future studies would be the immediate and direct investigation of transporters in the AT membranes from freshly biopsied tissue to further determine the (relative) contribution of these proteins. Finally, comparing regional fat uptake in women with gynoid to android fat distribution might provide additional information on the background of body fat distribution and the relative contribution of direct vs. VLDL-mediated FA uptake hereto. We are also attempting to investigate the effect of PIO on regional transport protein expression. 


\section{Hyperinsulinemia Abolishes Sex Differences In The Regulation Of Free Fatty Acid Release But Introduces Body Fatness As An Important Regulator}

Obesity leads to various abnormalities of FFA release from adipose tissue. These have been implicated and at times proven to mediate some of the metabolic abnormalities seen in obesity (65).

Under post-absorptive conditions, systemic FFA flux/ adipose FFA release has been shown to be greater in women than in men and to be best related to REE rather than FFM. In contrast, basal glucose production rates are equal in both sexes and related to FFM, but not to REE (66). Chapter 2.3 describes the additional effects of fitness on basal lipolysis and glucose flux, and reports on the major factors relating to adipose tissue FFA release and endogenous glucose production during hyperinsulinemia.

We found that moderate hyperinsulinemia abolishes the sex difference in FFA release: the relationship between plasma FFA flux ( $\mu \mathrm{mol} / \mathrm{min})$ and REE is maintained, but sex is no longer significantly related to FFA flux. Instead, body fatness is associated with greater FFA release, independently of REE. Fitness (peak $\mathrm{VO}_{2}$ ) does not influence FFA flux, neither under basal post-absorptive nor under hyperinsulinemic conditions.

Glucose flux, in contrast, is positively related to FFM, but, surprisingly, also to body fat. During hyperinsulinemia, peak $\mathrm{VO}_{2}$, plasma FFA and percent body fat additionally influence insulin stimulated glucose disposal independently

Figure 5.1 Correlations between Resting Energy Expenditure (REE) and Glucose Flux. Data from obese (circles), lean trained (asterixes) and lean sedentary (squares) are shown under basal (open symbols, left panel) and hyperinsulinemic (closed symbols, right panel) conditions. Regression lines of the three groups largely overlap and are almost identical in the two lean volunteer groups (left panel; $* \mathrm{P}<0.05$ ). Hyperinsulinemia separates the lines to (almost) parallel sublines; in obesity, correlations are largely lost, whereas in lean volunteers, the correlation remains, and shows a marked influence of fitness level $(* \mathrm{P}<0.05)$.

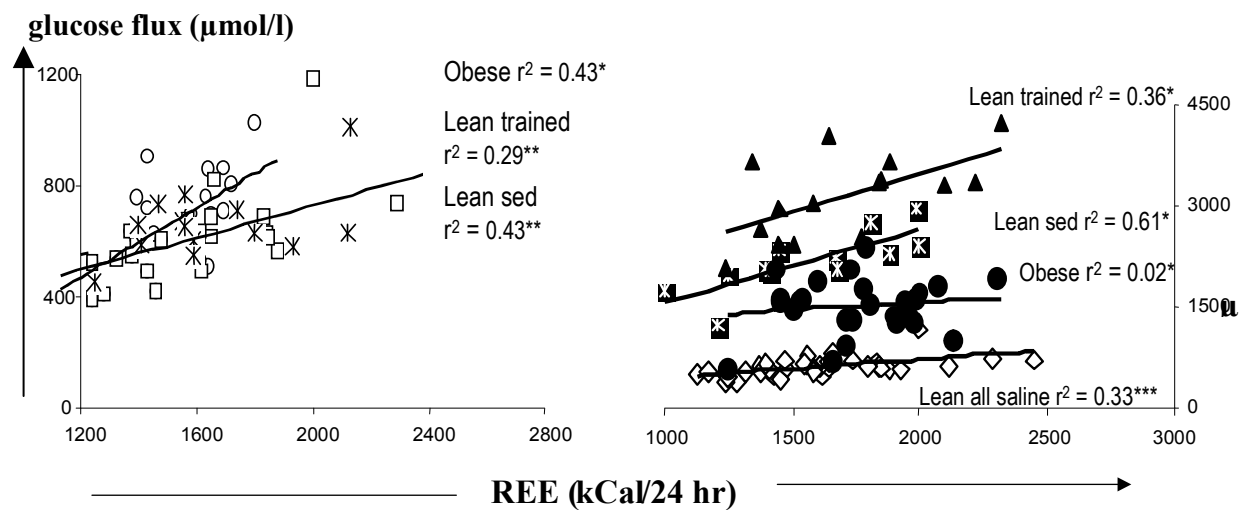

As stated in the Discussion (page 41), physical fitness improves metabolic function, but it does not do so via effects on basal or insulin suppressed lipolysis. Contrastingly, there is a marked correlation between fitness and glucose disposal. Figure 5.1 illustrates this nicely. The left panel demonstrates a great overlap between data from obese, lean trained and lean sedentary volunteers, which, in the latter two groups, are virtually identical. This is further indi- 
cated by the equations for the regression lines of the lean sedentary (glucose flux $=0.29 * \mathrm{REE}$ $+\mathrm{c}$ ) and the lean trained (glucose flux $=0,24 * \mathrm{REE}+\mathrm{d}$ ).

Hyperinsulinemia shifts the regression lines upwards, indicating higher glucose fluxes at similar REE levels, as expected. Both fitness and fatness influence this shift: it is most marked in trained volunteers and least in obese subjects. In the latter, the statistical significance is, lost; regression lines of lean trained (flux $=1.1^{*} \mathrm{REE}+\mathrm{e}$ ) and lean sedentary (flux $=1.1^{*}$ REE + f) subjects, however, remain statistically significant and remarkably parallel to each other and, although to a much lesser extent, to the saline line (flux $=0.3 * \mathrm{REE}+\mathrm{g}$ ).

In the discussion, we explain the implications of our findings on the interpretation of previous data (in which glucose flux is many times expressed as a function of FFM) and future studies.

\section{Obesity and its influence on endocrine control of fuel partitioning}

The balance between energy intake and expenditure greatly influences human health, as does the trafficking of different nutrients within the body and the cells. The latter process, in which the organism selects fuels for storage or oxidation, is known as 'nutrient partitioning' and may be influenced by several variables, among others hormones. The latter exert their influence on various levels and through various mechanisms, among others via the modulation of FFA metabolism.

After an overview on fuel (carbohydrate, fat and protein) processing and energy expenditure, Chapter 3.2.1 discusses the effects of insulin, GH, testosterone, estrogens and glucocorticoids respectively on several aspects of nutrient partitioning. These consist of resting energy expenditure and the thermic effect of food and exercise, substrate availability and oxidation, regional fat storage. Subsequently, the pathophysiological changes in (visceral) obesity and, where applicable, the therapeutic administration of these hormones are evaluated.

This chapter demonstrates why, in certain cases, results of endocrine tests must be interpreted differently in obese than lean subjects. It also provides a basis for understanding some of the points made in Chapter 4.

\section{Pioglitazone is associated with increased peripheral clearance of plasma free fatty acids, and with virtually no additional suppression of lipolysis}

TZD influences on in vivo FA kinetics are poorly understood and therefore at times difficult to put into perspective. For instance, not all TZDs lower plasma TG (and not in all conditions), whereas they all lower plasma FFA concentrations (281,369-371).

Chapter 4.5.3 describes FFA trafficking before and after PIO treatment, contrasted to Diet/ex. In the 39 UBO volunteers described in Chapter 4.5.2, FFA flux was measured using ${ }^{3} \mathrm{H}$-oleate disappearance rates under post-absorptive and hyperinsulinemic conditions. Both interventions lowered plasma FFA concentrations; however, post-Diet/ex this resulted from decreased lipolysis rates, whereas post-PIO, it was due to a $\sim 40 \%$ increase in FA clearance from the circulation. This was especially, but not exclusively, seen during hyperinsulinemia. Post-PIO, the anti-lipolytic efficiency of insulin improved, but absolute lipolysis rates remained unchanged. Because the increase in FFA clearance was not seen after Diet/ex intervention, this observation was unlikely to have been caused by insulin sensitization per se, but probably directly by PIO. This increased FFA clearance is non-oxidative. 
In our discussion (page 118), we discuss possible reasons for the discrepant findings of previous reports of decreased lipolysis rates after TZD administration. The above-mentioned difference between insulin resistant versus type 2 diabetic subjects and the possible confounding by improved glycemic control seems particularly relevant in this respect. It would therefore be interesting to repeat these studies in Type 2 diabetic subjects and perhaps even in healthy volunteers to further clarify these questions regarding TZD-influences on FFA clearance.

Despite the lack of human studies confirming our findings, rodent studies have indirectly demonstrated direct TZD influences on FFA clearance. In mice which completely lack AT, rosiglitazone did not increase insulin sensitivity, but did decrease plasma FFA concentrations (244). Since this could not have resulted from increased anti-lipolysis, it must have resulted from increased FFA efflux from the circulation, i.e. FFA clearance. This study also indicates that influencing plasma FFA concentrations and clearance is a direct TZD feature, occurring independently from both insulin sensitization and anti-lipolysis.

In the discussion, we also speculate on the tissues responsible for increased nonoxidative FFA clearance by TZDs. Since whole body FFA oxidation did not increase and TZDs have been demonstrated to decrease fat contents of myocytes and hepatocytes, the latter two tissues are virtually excluded (259), unless VLDL production would increase (see Chapter 4.5.3). Since neither TG concentrations nor VLDL-cholesterol changed significantly ( $\sim 31 \mathrm{vs} 30 \mathrm{mg} / \mathrm{dl})$, this would require a simultaneous increase in VLDL clearance. We have no evidence of that.

Previous publications have also shown that PIO does not alter production or removal of VLDL particles, but does increase the fractional clearance rate of VLDL-TG from the circulation (297). This is compatible with the decreasing VLDL particle size in our data (Chapter 4.5.5), but not necessarily with the unchanged TG concentrations.

Increased non-oxidative FFA clearance could further -theoretically- result from increased direct FFA uptake into AT (see above and Chapter 2.2). This, in turn, could involve changes in FA transporter-expression. Indeed, TZDs have been shown to amplify FFA transporter protein expression and synthesis in femoral subcutaneous fat, but not in skeletal muscle $(372,373)$. If there would be a regional difference herein, it might even concord with the altered fat distribution observed with TZD treatment.

These items, however, still remain speculative and would require further research.

\section{We Find Little Support For A Central Endocrine Role For Resistin, TNF- Alpha And IL-6 In Upper Body Obesity}

The production of adipokines by adipose tissue in quantities directly or inversely (adiponectin only) paralleling fat mass (Chapter 2.1.4) has led to the theory that some of these may impact insulin resistance via endocrine mechanisms. However, it is unclear whether the correlations are mainly with insulin sensitivity or fat mass only.

We took advantage of the fact that our study design of Chapter 4.5.2 involved two methods of insulin sensitization with opposite effects on fat mass: expansion (PIO) vs. reduction (Diet/ex). Focusing on resistin, adiponectin, interleukin-6 (IL-6), tumor necrosis factor- $\alpha$ (TNF- $\alpha$ ) and C-reactive protein (CRP), we assessed the relationship amongst plasma adipokine concentrations and their relationship with insulin sensitivity/body composition, before and after the respective interventions. Besides those correlations, we hoped to gain insight into the distinction between fat mass effects versus insulin sensitization effects.

Chapter 4.5.4 reports the outcomes of this assessment. Plasma adipokine concentrations were not consistently interrelated (except the cytokines and CRP), and only adiponectin displayed 
the expected relationship with insulin resistance and sensitization. Not only plasma concentrations of adiponectin, but also its associations with fatness/insulin sensitivity were different in men and women. PIO increased adiponectin substantially more $(+3581 \mathrm{ng} / \mathrm{ml}, \mathrm{p}<0.001)$ than did Diet/ex $(+722 \mathrm{ng} / \mathrm{ml}, \mathrm{p}<0.01)$.

CRP decreased only with diet/exercise, whereas TNF- $\alpha$, IL-6 and resistin did not respond to either intervention. The cytokines and CRP were correlated with fatness more than with insulin sensitivity, but were not statistically significantly related to either in most cases.

IL-6 and TNF- $\alpha$ exert a vital role in stress, inflammatory and immunological responses of the body. Given the importance of fuel (particularly FFA-) partitioning herein (Chapter 3.2.1), it is not surprising that these markers should have a consistent correlation with important energy depots, and with AT in particular. Effects of the cytokines on AT might be paracrine, autocrine or even pivotal ${ }^{1}$.

However, the very centrality of their role in basic metabolic processes, most of which are not exclusive to adiposity/insulin resistance, complicates research of these markers in this context and increases the possibility of confounding factors. Moreover, it makes one wonder about the likelihood of their central involvement in insulin resistance. The latter would imply that, in case of stress or inflammation, the body would automatically fall back into a state of insulin resistance.

Nevertheless, this is not inconceivable. Cortisol, the stress hormone par excellence, also exerts marked insulin desensitizing effects (Chapter 3.2.3.5) and one might therefore even consider the option that a certain degree of insulin resistance is an endogenous necessity for stress responses. However, a central and chronic endocrine role in mediating insulin resistance in people who are not in a state of physiological stress is difficult to integrate with most study results. Why would men and women have different levels of CRP at a similar BMI? (see below). If adipokines were central mediators, wouldn't we have expected their concentrations and correlations with insulin sensitivity parameters to at least change consistently and in the expected direction after insulin sensitization, even if not statistically significantly?

Although we acknowledge the limitations posed by our small sample size, and the preselection of insulin resistant volunteers (see below), it is difficult to ascribe our observations to inadequate statistical power. An important endocrine effect of adipokines (arbitrarily defined as explaining at least $25 \%$ of the variance in insulin sensitivity measures) should be detectable with 39 subjects. From a clinical endocrinology perspective, hormone effects explaining $<10 \%$ of the variance in the response are not considered powerful. In our study, the relationship between adiponectin and $\mathrm{Si}$ was such that $10-16 \%$ of the variance could be explained/detected. In contrast, some of the correlations between other adipokines and insulin sensitivity (especially resistin) were in opposite directions of that predicted. If the adipokines were half as powerful as other known indices of insulin action (fatness, free fatty acids, fasting plasma insulin concentrations, etc) the effect would have been readily detectable as evidenced by the associations we did detect (Chapter 4.5.6).

To further avoid random, coincidental correlations we also performed a principal component analysis. This too might have suffered from our sample size, but showed a marked consistency with the Pearson's correlations we ran afterwards.

\footnotetext{
${ }^{1}$ acute vs. chronic elevations of IL-6, which may have divergent effects, causing insulin sensitivity and insulin resistance respectively (21)
} 
We therefore feel that it is safe to state that our findings therefore do not support a central endocrine role for most adipokines, except adiponectin, in mediating insulin resistance. Smaller roles or autocrine/paracrine roles have been neither contradicted nor confirmed by our results.

\section{Adiponectin is associated with insulin sensitivity parameters and fatness in a gender-dependent manner, analogously to its plasma concentrations}

Adiponectin was the only adipokine that consistently correlated with parameters of both insulin sensitivity and fatness. The gender difference in its concentrations at similar levels of insulin resistance and BMI, however, remains puzzling. The suggested explanation for similar findings in CRP, namely that higher fat loads in women at similar weights/BMIs would lead to higher plasma CRP concentrations, cannot be extrapolated to (and is even inconsistent with) this observation.

The ratio of high-to-low molecular weight adiponectin isoforms might partly explain the gender mystery. It has been suggested that this ratio, not absolute adiponectin concentrations, determines insulin sensitivity (374); testosterone has been shown to selectively reduce adipocyte secretion of the high molecular weight form $(375,376)$. This could explain gender differences in adiponectin concentrations at baseline as well as in response to Diet/ex. This, however, does not shed light on the difference seen in CRP.

TZDs are also thought to influence this ratio (377). This, in turn, does not explain why PIO increased absolute adiponectin concentrations more than could be accounted for by the mere insulin sensitization. The latter suggests yet another direct PIO-effect, independent of insulin sensitization.

It would therefore be interesting to re-examine the correlations described in Chapter 4.5.4 after distinguishing high from low molecular weight adiponectin isoforms in order to see whether and, if so, how, sex differences would remain apparent, and to what extent this ratio influences insulin sensitivity in this population after Diet/ex and PIO treatment. As with most TZD influences, it would further be interesting to examine the effects of PIO administration to healthy individuals on adiponectin concentrations and isoforms.

\section{Pioglitazone increases LDL Particle Size irrespective of changes in triglyceride concentration. This increase is not directly related to insulin sensitization}

Small, dense LDL particles are possibly associated with unfavorable cardiovascular outcomes (as opposed to larger-size particles). However, this is difficult to investigate: a small LDL pattern usually occurs simultaneously with other cardiovascular risk factors, such as unfavorable lipid profiles including high TG and low HDL cholesterol levels, the metabolic syndrome, insulin resistance and type 2 diabetes. In addition, lowering plasma TG concentrations inherently induces a reshuffling of lipid particles with an increase in LDL particle size. As insulin sensitization intrinsically reduces plasma $\mathrm{TG}$, this in turn complicates the study of any direct effects of interventions involving insulin sensitization on LDL particle size.

Previous studies of TZD effects on LDL particle size do not cover all these concerns and are additionally confounded by the potential influence of improved glycemic control in type 2 diabetic patients. 
In our study of non-diabetic volunteers (protocol of Chapter 4.5.2), PIO-induced insulin sensitization did not lead to TG lowering (Chapter 4.5.6). This allowed us to analyze the effect of PIO on particle reshuffling uncoupled from TG lowering; comparing these to Diet/exercise could additionally distinguish non-specific insulin sensitizing effects from 'idiosyncratic' characteristics of PIO. We used the relatively new technique of NMR to determine particle size.

Chapter 4.5.5 demonstrates that both treatments decreased the amount of total and small LDL particles and increased large LDL particle concentrations. However, only Diet/ex also reduced TG, total cholesterol and absolute LDL concentrations, and thus total LDL particle concentration. Consistently herewith, LDL particle subclass changes correlated with changes in $\mathrm{Si}$ in the Diet/ex, but not the PIO group, even after correction for the change in TG. Thus, these two approaches to insulin sensitization had similar effects on LDL particle size, yet distinctly different patterns of achieving that response.

Quantitative measures of LDL cholesterol alone might therefore not reflect the full 'lipid burden'. However, it remains unclear to what extent variations in particle size have a true independent influence on the clinical risk of developing cardiovascular diseases: this factor might easily be overwhelmed by other cardiovascular risk factors such as smoking, overweight and hypertension. If one could a find a group of (healthy) volunteers with small LDL particle sizes, but without other important risk factors for CVD, one might study the effect of size modulation on the development of CVD. Unlikely as it is to find such a group, this study could in turn be easily confounded by the effect of the means of altering particle size.

Still, from a pathophysiological standpoint it is interesting to see that, in vivo, influencing LDL particle size can, indeed, be uncoupled from TG concentrations and from insulin sensitization, and that PIO influences this process. TZDs also influence several other aspects of lipid metabolism directly, such as LPL $(297,355)$, and perhaps also hepatic lipase action (356), FA transporter protein expression and, as shown above, FFA clearance. These effects seem to be unrelated to insulin sensitization and might perhaps contribute to some of the divergent TZD effects on lipids despite their similar insulin sensitizing effects. Interestingly, rosiglitazone effects on LDL subfractions are variable, showing either a general increase (348) or an increase in large LDL only (378). Also, the combination of PIO and Glimepiride or Metformin reduced plasma Lp(a) levels, whereas the same combinations with rosiglitazone did not $(379,380)$.

\section{Does pioglitazone improve non-alcoholic fatty liver disease?}

In Chapter 4.4.3 it is suggested that PIO has a positive influence on non-alcoholic fatty liver disease (NAFLD) in non-diabetic volunteers. Contrary to the idiosyncratic hepatoxicity of TZDs, we coincidentally found that PIO improved biochemical indices of non-alcoholic fatty liver disease (NAFLD) in all our subjects in which this was present.

Although we only had 5 study subjects in which this was the case, and it was a coincidental, post-hoc finding, our observation was consistent with previous reports on troglitazone and rosiglitazone (252) in type 2 diabetic volunteers, and was the first to suggest these effects in PIO and in non-diabetic UBO subjects. Later, our suggestion was confirmed in Type II diabetic (381), (382) and non-diabetic subjects $(383,384)$, the latter two studies including histological evidence.

An important implication of this TZD characteristic is the possibility to treat subjects, who would otherwise not receive treatment, neither for NAFLD nor for insulin resistance. In- 
terestingly, insulin sensitization using metformin, like TZDs, postpones the development of type 2 diabetes (227), but does not affect liver fat content (300). TZDs have been suggested to do both $(238,239)$, and some suggest that (TZD) treatment of insulin resistance should be considered before the occurrence of DM (385).

More prospective research, however, is needed to evaluate whether the use of TZDs as a treatment option for NAFLD per se should be encouraged. It is especially unclear whether TZDs would also be effective in reducing NAFLD in other patient categories, particularly since the above-mentioned studies do not give pathophysiological insight in the mechanism of action of TZDs and of the pathogenesis of NAFLD. Not only the role of direct PPAR ( $\gamma$ or $\alpha$ subtype) stimulation, but also the decrease in insulin and FFA levels may be important topics of future investigation. Since increased insulin (and FFA) concentrations have been suggested to be crucial factors in the development of NAFLD, one might consider the additional decrease of these compounds on top of the effect of insulin sensitization per se (e.g. via increased FA clearance- see above) (386).

The natural fluctuation of this notoriously focal disease, however, complicates its research.

\section{Responsiveness of adipose tissue to TZD is site-specific: PIO selectively enhances femoral fat accretion}

Chapter 4.5.6 focuses on the effect of PIO versus Diet/ex induced insulin sensitization on fat distribution. In the 39 UBO volunteers who completed our study program (Chapter 4.5.2), Diet/ex treatment reduced whole body fat with a preferential reduction of abdominal (visceral more than subcutaneous) adipose depots. It therefore lowered the waist-to-hip ratio (WHR).

PIO treatment also improved insulin sensitivity and lowered WHR; however, the latter resulted from a selective increase in lower body fat without changes in upper body fat (including the visceral depot). The findings from WHR measurement, CT and DEXA scanning are supported by consistent parallel microscopic changes in fat cell size, although the latter did not always reach statistical significance.

This confirms that responsiveness of adipose tissue to TZD is site-specific, also in vivo, and suggests that improvements in whole-body insulin sensitivity by PIO are achieved independently of changes in intra-abdominal fat volume. Changes in regional fat compartments do not directly correlate with changes in insulin sensitivity parameters.

These findings contrast with the general idea that TZDs decrease visceral fat amounts.

However, as mentioned in our discussion (page 148), closer investigation of the available literature show that the effect of TZDs on visceral fat is very variable too and is mostly studied in type 2 diabetic patients.

Table 5.1 summarizes these (updated) data. The one available report on UBO, non-diabetic volunteers confirms a decrease in waist-to-hip ratios without altered waist diameters with pioglitazone (383). We suggest that the choice of non-diabetic vs. type 2 diabetic volunteers may partly account for the discrepancy in these findings.

Our results also suggest that the undoubted importance of visceral fat is only one of many determinants of insulin resistance. Lower body fat, in contrast, has been suggested to exert an independent protective metabolic effect $(387,388)$ even if this might perhaps be overridden by the deleterious influence of abdominal adiposity (389). 
Table 5.1 Effect of thiazolidinediones on fat distribution

\begin{tabular}{|c|c|c|c|c|c|c|c|c|c|c|}
\hline Author & $\mathbf{n}$ & $\begin{array}{l}\# \\
\text { wks }\end{array}$ & $\begin{array}{l}\text { Control } \\
\text { group }\end{array}$ & Therapy & Method & weight & $\begin{array}{l}\text { Total } \\
\text { Fat }\end{array}$ & $\begin{array}{l}\text { VISC } \\
\text { fat }\end{array}$ & $\begin{array}{l}\text { ASC } \\
\text { fat }\end{array}$ & $\begin{array}{l}\mathrm{V} / \mathrm{s} \\
\text { ratio }\end{array}$ \\
\hline $\begin{array}{l}\text { Akaza- } \\
\text { wa (361) }\end{array}$ & 20 & 52 & No & $\mathrm{T}+\mathrm{SU}$ & $\mathrm{CT}$ & $\uparrow$ & NR & $\equiv$ & $\uparrow$ & $\downarrow$ \\
\hline $\begin{array}{l}\text { Basu } \\
\text { (391) }\end{array}$ & 8 & 12 & glipizide & P $45 \mathrm{mg}$ & $\begin{array}{l}\text { DEXA } \\
+\mathrm{CT}\end{array}$ & $\uparrow(\mathrm{NS})$ & $\begin{array}{l}\uparrow \\
(\mathrm{NS})\end{array}$ & $\begin{array}{l}\equiv / \downarrow \\
(\mathrm{NS})\end{array}$ & $\mathrm{NR}^{1}$ & \\
\hline $\begin{array}{l}\text { Carey } \\
(392)\end{array}$ & 33 & 16 & Placebo & Rosi & $\begin{array}{l}\text { MRI } \\
+ \text { DEXA }\end{array}$ & $\uparrow$ & $\uparrow^{2}$ & $\equiv$ & $\uparrow$ & NR \\
\hline $\begin{array}{l}\text { Hirose } \\
\text { (393) }\end{array}$ & 10 & 12 & none & $\begin{array}{l}\text { P } 30 \mathrm{mg} \\
+ \text { diet } \pm \mathrm{SU}\end{array}$ & $\mathrm{CT}$ & $\uparrow$ & NR & $\uparrow(\mathrm{NS})$ & $\uparrow$ & $\begin{array}{l}\downarrow \\
\text { (NS) }\end{array}$ \\
\hline $\begin{array}{l}\text { Kelly } \\
(260)\end{array}$ & 11 & 12 & 10 placebo & $\begin{array}{l}\mathrm{T} 600 \mathrm{mg} \\
\text { various } \\
\text { co-meds* }\end{array}$ & MRI & $\uparrow(\mathrm{NS})$ & $\equiv^{3}$ & $\downarrow$ & $\downarrow^{4}$ & $\mathrm{NR}$ \\
\hline \multirow{2}{*}{$\begin{array}{l}\text { Mori } \\
(362)\end{array}$} & \multirow{2}{*}{$\begin{array}{l}30 \\
\text { lean }\end{array}$} & \multirow[t]{2}{*}{26} & \multirow[t]{2}{*}{ No } & $\operatorname{diet}+\mathrm{T}$ & \multirow[t]{2}{*}{ CT } & $\uparrow$ & NR & $\downarrow$ & $\uparrow$ & $\downarrow$ \\
\hline & & & & $\begin{array}{l}\operatorname{diet}+\mathrm{T}+ \\
\text { SU }\end{array}$ & & $\uparrow$ & NR & $\equiv$ & $\uparrow$ & $\begin{array}{l}\downarrow \\
\text { (NS) }\end{array}$ \\
\hline \multirow{3}{*}{$\begin{array}{l}\text { Miya- } \\
\text { zaki } \\
(281) \\
\text { Kawai } \\
(360)\end{array}$} & 13 & \multirow[t]{3}{*}{16} & No & $\begin{array}{l}\text { Pio } 45 \\
+ \text { SU or } \\
\text { diet }\end{array}$ & MRI & $\uparrow$ & \multirow[t]{3}{*}{$\uparrow$} & $\downarrow$ & $\uparrow$ & $\downarrow$ \\
\hline & $\begin{array}{l}48 \\
3\end{array}$ & & Diet only & $\operatorname{diet}+\mathrm{T}$ & CT & NR & & $\begin{array}{l}\downarrow(\mathrm{SC} \uparrow, \\
\mathrm{NS})\end{array}$ & $\equiv$ & $\downarrow$ \\
\hline & only & & & $\begin{array}{l}\operatorname{diet}+\mathrm{T}+ \\
\text { SU }\end{array}$ & & & & $\equiv$ & $\equiv$ & $\equiv$ \\
\hline $\begin{array}{l}\text { Katoh } \\
\text { (261) }\end{array}$ & 78 & 28 & untreated & $\mathrm{T}+\mathrm{SU}$ & CT & NR & & $\equiv^{5}$ & & $\downarrow$ \\
\hline $\begin{array}{l}\text { Smith } \\
\text { (394) }\end{array}$ & 48 & 24 & placebo & $\begin{array}{l}\text { Pio } 45 \mathrm{mg} \\
+ \text { ? }\end{array}$ & $\begin{array}{l}\mathrm{CT}+ \\
\text { DEXA }\end{array}$ & $\uparrow$ & $\uparrow{ }^{6}$ & $\equiv$ & $\uparrow$ & \\
\hline $\begin{array}{l}\text { Virta- } \\
\text { nen } \\
(395)\end{array}$ & 41 & 26 & metformin & $\begin{array}{l}\text { Rosi } 2 \text { x } 4 \\
\text { mg }\end{array}$ & MRI & $\equiv$ & NR & $\begin{array}{l}\downarrow \text { both } \\
\text { groups }\end{array}$ & & NR \\
\hline $\begin{array}{l}\text { De Souza } \\
(363)\end{array}$ & Z & 4 & $\begin{array}{l}\text { Lean }+ \\
\text { obese }\end{array}$ & Pio & $\begin{array}{l}\text { MRI/ } \\
\text { post } \\
\text { mortem }\end{array}$ & $\uparrow$ & $\uparrow$ & $\uparrow 8$ & $\downarrow$ & $\equiv$ \\
\hline
\end{tabular}

$\mathrm{T}=$ Troglitazone; Pio=Pioglitazone; Rosi= rosiglitazone; *not specified; Abd: abdominal; NR=not reported; $\mathrm{NS}=$ non-significant; SC:subcutaneous; VISC: visceral; Z=Zucker rats

Although perhaps counter-intuitive, the concept of fat being metabolically protective is compatible with the observation that the absence of fat in itself induces marked insulin resistance (such as in lipoatrophic disorders), and in addition blocks the insulin sensitizing action of TZDs in mice $(244,390)$.

\footnotetext{
${ }^{1}$ Total abdominal fat reduced by $6 \%$ (NS)

$295 \%$ of which in non-abdominal region

${ }^{3}$ Placebo group lost fat too, but less than T group

${ }^{4}$ Waist circumference increased, and WHR decreased; both NS compared to placebo

${ }^{5}$ absolute decrease was non-siginificant; the difference from baseline was significantly greater than in the placebo group.

${ }^{6}$ incl leg, arm and trunk fat

${ }^{7}$ Insulin sensitivity $\uparrow$ in non-Abd SC, not in VISC AT

${ }^{8}$ Greatest $\uparrow$ in ovarian fat, more than retroperitoneal or SC abd fat
} 
Furthermore, it has been reported that, even in lower body obesity phenotypes, the majority of circulating FFAs originate from upper body fat depots $(\sim 70 \%$, vs. $\sim 20 \%$ from the leg (47)). The latter implies that LBO is associated with a better insulin sensitivity than UBO despite similar total FFA loads; this would be compatible with a compensating or 'protective' factor coming from leg fat. The concept would also be consistent with the metabolic improvement found by PIO in the presence of increasing (lower body) fat mass.

Yet the actual effects of TZDs on fat metabolism and insulin sensitization might be unrelated to fat distribution. Studying TZD-like compounds with a lesser effect on fat mass, perhaps by bypassing the AT-selective PPAR- $\gamma_{2}$ and/or selective stimulation of the PPAR- $\gamma_{1}$ receptor might provide further insight herein. Studying lean, healthy volunteers may add additional insight.

\section{Insulin sensitization is superior to several other treatment options of UBO}

Given the labor intensity and low success/high relapse rate of weight loss regiments, several other therapeutic options have been examined for the treatment of the metabolic complications of visceral obesity. Chapters 4.2 and 4.3 discuss administration of testosterone and $\mathrm{GH}$ respectively in upper body obese men (GH also in women), based on the observation that $\mathrm{GH}$ and testosterone concentrations are lowered in these groups. We review the results of the available literature on metabolic parameters and body composition and conclude that GH does not convincingly alter body composition and seems to deteriorate certain metabolic functions; among others, it has been proven to induce insulin resistance, the prevention of which was one of the initial reasons to administer $\mathrm{GH}$.

This lack of overall metabolic improvement (and even worsening) with GH administration is compatible with our view that the changed concentrations of $\mathrm{GH}$ and testosterone in UBO must be viewed as adjustments of the body to the state of energy surplus.

We therefore, in general, advise against their therapeutic use for this indication. Insulin sensitization methods using diet and exercise and pioglitazone are discussed in Chapters 4.4 and 4.5 .

\section{TZD therapy should not substitute for lifestyle modification in the insulin-sensitizing treatment of obesity}

It is unclear to what extent the TZD- induced insulin sensitivity and other effects compare quantitatively to the 'gold standard' of Diet/ex. If equally effective, TZD administration might gain in attraction since its labor-intensity is markedly lower for both patient and doctor.

Chapter 4.5.6 also includes a comparison between the effects of 19 weeks of Diet/ex vs. PIO on insulin sensitivity, metabolic health and fitness parameters. In 39 UBO volunteers, PIO was similar to Diet/ex in its effects on insulin sensitivity parameters, but inferior in cholesterol and TG lowering. The slight inferiority in the increase in the sensitivity index $\mathrm{Si}$ in the PIO group did not reach statistical significance. Diet/ex, but not PIO, additionally improved fitness parameters and reduced weight, both of which are expected to have extra longterm protective effects on (non-metabolic) consequences of obesity.

Does the fact that Diet/ex improve metabolism and fitness more than PIO administration imply that Diet/ex should be favored in clinical practice? One may argue that a $\sim 12 \mathrm{~kg}$ average weight loss in 20 weeks in a class I obese population is not easily achieved in average clinical 
practice, and that the benefits of Diet/ex might not outweigh those of TZD treatment in case of more modest (and perhaps more common) weight loss. A study comparing $7 \mathrm{~kg}$ weight loss (in $\sim 20$ weeks) to rosiglitazone administration in UBO non-diabetic volunteers (396) did, unfortunately, not provide the answer, since fitness and lipid parameters were not reported; improvement of insulin sensitivity parameters was similar with the two regiments.

Do both interventions prevent diabetes in prediabetic individuals? Definitive evidence for TZDs is still lacking, although there are sme indications that it might do so $(238,239)$. Lifestyle intervention, however, has been proven to reduce progression rates to diabetes in the $\mathrm{Da}$ Qing Study (by 40\%), the Finnish Diabetes Prevention Study (by 58\%) and the Diabetes Prevention Program (by 58\%); it is furthermore claimed to be cost-effective when administered chronically (229).

Is there an additional benefit from the non-insulin-sensitizing effects of TZDs? As discussed in Chapter 4.4.2, these include adiponectin increment (see below), blood pressure lowering, decreasing of intima medial thickness (247) and a suggested beneficial influence on several forms of (obesity-associated) cancer (397,398); however, the clinical applications hereof are still unclear and might require TZD administration for as long as obesity is present. Moreover, most of these benefits have also been reported for lifestyle intervention, although, here too, the exact clinical relevance and/or possible quantitative differences herein is not always clear.

Several non-metabolic complications such as osteoarthritis, sleep apnea etc, are not known to be reduced by TZDs.

Therefore, in our view, TZD therapy should not substitute for lifestyle modification in the treatment of high-risk obesity.

\section{TZD therapy: efficacy versus safety}

The safety of TZD administration has been subject to debate. Hepatotoxicity issues have been resolved after the retraction of troglitazone (see Chapter 4.4.2) and after adverse liver effects in PIO and rosiglitazone appeared to be mild and reversible (248-251). Nevertheless, it is unclear what percentage of treated patients the latter involves, and it is still recommended to monitor liver enzymes in the initial phases of TZD administration. TZDs are now thought to have beneficial effects on steatotic livers (see above), although the administration for this indication has not been officially introduced.

An unwanted side effect of TZD therapy is fat and weight gain. Even if this does not prevent metabolic improvement, it is an unfortunate issue for most patients, especially those who have been urged to lose weight to improve their insulin sensitivity. It is unknown whether combining TZDs with Diet/ex would overcome this deleterious influence, and whether insulin sensitizing effects of the two would augment, neutralize or overrule each other. Of note, addition of metformin to lifestyle intervention had no additive effect (The Indian Diabetes Prevention Programme (231)).

Weight gain is likely mediated by both fat accretion (Chapter 4.5.6) and extra- and intravascular fluid retention caused by TZDs. The latter poses a realistic safety concern, since in some, but not all studies (399-401) this seemed to increase the risk of heart failure in diabetic patients $(237,402)$. Whether this is counterbalanced by beneficial TZD influences, e.g on glycemic control, blood pressure (347), vascular function (403), vasodilatation and left ventricular diastolic function (404) is unclear; however, overall mortality, nonfatal myocardial infarc- 
tion and stroke rates did decrease after adding $45 \mathrm{mg}$ PIO to conventional anti-diabetic therapy.

Several reports hereof, however, including the largest (PROactive study), were confounded by, among others, the combination of TZDs with insulin, which notoriously increases heart failure and other adverse cardiovascular events (405). A sub-analysis distinguishing these cases from those where insulin was not used might provide valuable extra information regarding the issue of TZD-induced heart failure. Despite this and several other important limitations to this study, such as its relatively short duration, it remains the only prospective study of the effects of TZDs on heart failure and other cardiovascular end points.

One could argue, as did Maru et al (406), that the severity and the duration of diabetes might be a better explanation for heart failure than the use of TZDs itself. Nonetheless, the PROactive study underscores the AHA recommendation to refrain from prescribing TZDs in NYHA class III and IV heart failure and to be cautious in patients with previous heart diseases (407).

\section{Limitations}

Various limitations to our studies have been described in the discussions of the respective sub-studies to which they apply; however, several general limitations with a possible impact on all our papers can be pointed out.

\section{Selection of volunteers}

Our volunteers were specifically selected to be obese and insulin resistant for reasons described in Chapter 4.5.1. This choice narrowed the range in several parameters, such as visceral fat mass, and, to a lesser extent Si. In some cases, this may have reduced the strength of certain correlation coefficients, e.g. between $\mathrm{Si}$ and anthropometric/body composition parameters, or Si and our adipokine analyses (see Chapters 4.5.4 and 4.5.6).

The relatively small sample size is another limitation. Although it is very challenging to include more than 39 subjects in a human clinical-physiological study, a larger sample size might have limited the influence of, e.g., intrinsic inter- and intra-individual differences in measured parameters, both at baseline and in response to diet/ex and PIO. We therefore consistently checked the intrinsic consistencies of data from outliers to avoid confounding on this ground, and doubled, tripled or even quadruplicated several analyses, which were notoriously biologically variable.

We have additionally applied extra analyses such as Bonferroni analyses and the Principal Component Analysis (Chapter 4.5.4) to limit the likelihood of erroneous conclusions. Although the sample size may, in turn, have influenced the statistical power of the PCA, there is no consensus on whether absolute sample size or the ratio of subjects to items has the greatest influence hereon ${ }^{1}$.

Furthermore, we tried to counterbalance the limited number of participants with relatively accurate and precise measures of outcome. The best examples hereof are the assessment of body composition (DEXA and CT combined as opposed to the inferior bio-electric impedance analysis, DEXA only, skin fold thickness or waist circumference measurements) and FFA trafficking (isotope tracer studies and HPLC analysis).

\footnotetext{
${ }^{1}$ Osborne, Jason W. \& Anna B. Costello (2004). Sample size and subject to item ratio in components analysis. Practical Assessment)
} 


\section{Research Methods}

Nonetheless, a measurement that intrinsically lacks precision is the IVGTT. Since performing a euglycemic clamp (the gold standard) could, however, not be performed in all volunteers to assess their insulin action, we chose to use IVGTT as a 'second best' as it is considered superior to HOMA analysis (401) and to static parameters such as fasted glucose, insulin and Cpeptide only.

The dose of the insulin infusion during the clamp is another variable, which might have influenced the outcomes. We discuss the rationale for our choice in paragraph 4.5.2.2. In summary, we aimed to obtain plasma insulin concentrations high enough to detect an effect on FFA metabolism, but low enough to prevent complete suppression/ blockage of important processes. Indeed, our resulting insulin concentrations as well as the suppression of endogenous plasma oleate concentrations nicely reflect postprandial metabolism and are comparable to those following consumption of a mixed meal in obese adults (38). Nevertheless, a different insulin dose might have lead to different observations.

The use of ${ }^{14} \mathrm{C}$-oleate and palmitate tracers requires extrapolation to total FFA metabolism and therewith introduces intrinsic risks of inaccuracy. A recent re-evaluation of this principle, however, showed that palmitate, oleate, and linoleate tracers, indeed, reflect the rate of appearance (Ra) of total FFA within $15 \%$ of the measured sum of the individual fatty acid $\mathrm{R}(\mathrm{a})$, as opposed to stearate and myristate tracers (272). The effect of a euglycemic, hyperinsulinemic clamp hereon was either small or insignificant.

\section{Interventions}

Our interventions were monitored but unsupervised. Although this reflected clinical practice better, it lead to inter-individual differences in the type and intensity of the exercise program as well as to opportunities for dietary and exercise non-compliance, both in the Diet/ex and PIO groups.

Although this simultaneously provided the advantage of an increased range of assessed parameters, it might also have influenced our outcomes: insulin resistance and FFA metabolism may differ between situations of weight stability, weight loss and weight gain, and the various volunteers might have been in different phases of weight loss by the time we performed the second study. In other words, the amount of weight loss and/or exercise might have been greatest in the beginning, the middle or the end of the intervention period. We attempted by limiting the variability and influences of the latter effect by a gradual increase in the intensity of the exercise program, by monitoring both dietary progress and exercise logs and by 'guaranteeing' weight stability one week before the actual measurements (Chapter 4.5.2.2).

In the PIO group, we specifically asked our volunteers not to change their diet and life style during the study course. However, we realize that this could not always be achieved, if only due to the large seasonal climate fluctuations in Minnesota, which almost by default incited some differences in life style. A few volunteers lost weight $(\mathrm{n}=4: 0.6,1.1,1,4$ and 3,8 kgs respectively) despite PIO use (possible non-compliance was minimized by pill counts). However, practically all parameters of these volunteers were in line with those of the others PIO users; additionally, there was a wide overall range of post-PIO weight change, so that we have no reason to believe that this truly influenced our results. A larger study interval might have further reduced this problem.

Unfortunately, we were unable to study the reversal of the achieved results after discontinuing PIO treatment since the volunteers were also offered a complimentary diet/ex program after completing the study.

Finally, the post hoc nature of some research study questions (such as the particle sizes) ask for confirmation by future, prospective studies. 


\subsection{Nederlandse Samenvatting}

\section{Inleiding}

De exponentiële toename van obesitas wereldwijd neemt epidemische vormen aan. Het aantal hiermee geassocieerde complicaties zal de Nederlandse gezondheid(szorg) in de toekomst naar verwachting sterk beïnvloeden. Het moeizame karakter van grootschalige behandeling van overgewicht vergroot daarom het belang van inzicht in de pathophysiologie van obesitasgerelateerde metabole veranderingen, o.a in het kader van de ontwikkeling van eventuele nieuwe behandelingsopties hiervoor.

Dit proefschrift richt zich met name op insulineresistentie en vetzuurmetabolisme bij obesitas, en op de verbetering hiervan na insulinesensitisatie. Hierbij zullen de effecten van Pioglitazon (PIO) -therapie afgezet worden tegen dieet en lichaamsbeweging (Diet/ex). Met name bij eerstgenoemde bestaat nog niet veel inzicht in het werkingsmechanisme en de exacte metabole effecten; zo deze al bestudeerd zijn is dit gebeurd bij type-2-diabetespatiënten. Echter, door insulineresistente, 'upper body obese' (i.e. centraal obese, appelvormige) vrijwilligers te bestuderen in plaats van type-2-diabetici, omzeilden wij het confounding effect van een verbeterde glucoregulatie an sich op het functioneren van insuline. Om verder onderscheid te kunnen maken tussen de effecten van insulinesensitisatie enerzijds en specifieke effecten van PIO anderzijds, werd een gelijk aantal UBO-vrijwilligers behandeld met Diet/ex ter vergelijking. Behalve het bestuderen van interventies werden echter ook fysiologische aspecten van vetmetabolisme en obesitas onderzocht (Hoofdstuk 1-4).

\section{Samenvatting}

Hoofdstuk 1 beschrijft de obesitasepidemie en geeft een algemeen overzicht van het proefschrift. Hoofdstuk 2.1 is een korte synopsis van de huidige inzichten in vet- en vetweefselmetabolisme. Hoofdstuk 2.2 beschrijft een studie naar het bestaan van vetzuuropname in abdominaal en femoraal subcutaan vetweefsel zonder voorafgaande incorporatie in VLDLpartikels. Na validatie van de onderzoeksmethode werd met radioactieve vetzuurtracers en biopten uit buik- en bovenbeensvet aangetoond dat vrije vetzuren (FFA's) direct opgenomen konden worden in subcutaan vetweefsel, zonder voorafgaande incorporatie in VLDL of triglyceriden. Daarnaast bleken er evidente geslachtsverschillen te zijn: directe vetzuuropname was ca $70 \%$ efficiënter bij vrouwen dan mannen, maar bij mannen was de opname in abdominaal vet bovendien hoger dan in femoraal vet, wat niet het geval was bij vrouwen. Dit was het geval bij zowel obese als slanke vrijwilligers, en zowel bij kortdurende als langdurige (6 uur) tracertoediening.

Daarnaast blijkt mRNA expressie van transportproteïnen uit subcutaan vetweefsel overeen te komen met de gevonden regio- en geslachtsverschillen. 
De relevantie van deze bevindingen betreft de ontdekking van een nieuwe route van vetopname in vetweefsel. Deze werd tevoren onwaarschijnlijk geacht, omdat de netto efflux van FFA's uit vetweefsel in nuchtere toestand impliceert dat deze FFA's dus tegen de stroom in moeten zwemmen om opgenomen te worden. Onze data pleiten ervoor dat transporteiwitten dit faciliteren. Daarnaast kunnen onze bevindingen bijdragen aan het inzicht in verschillen in de metabool zo relevante vetverdeling tussen de geslachten, en wellicht zelfs tussen verschillende individuen. Aangezien er geen sexeverschillen zijn in FFA's die uit maaltijden origineren, lijkt er mogelijk een opening ontstaan te zijn voor het bestuderen hiervan.

Genoemde fenomenen bieden daarom nieuwe inzichten in de fysiologie van vetweefstel en de locale regulatie van de expansie hiervan. Beide zouden kunnen bijdragen aan het hogere percentage aan lichaamsvet alsmede de gynaecoïde vetverdeling bij vrouwen.

Hoofdstuk 2.3 beschrijft factoren die de afgifte en opname (flux) van endogene glucose en vetzuren beinvloeden. Bij obesitas is de 'handling' van deze substraten immers veranderd, met belangrijke gevolgen voor de metabole gezondheid. 137 studies bevestigden in 101 nondiabetische vrijwilligers, dat systemische vetzuurflux, i.e. lipolytische vetzuurrelease uit vetweefsel, groter is bij vrouwen dan mannen in nuchtere toestand, en dat deze daarnaast voornamelijk bepaald wordt door de Resting Energy Expenditure (REE). Hyperinsulinemie maakt de geslachtsverschillen ongedaan, maar introduceert 'lichaamsvet' als belangrijkste determinant van de FFA-release, onafhankelijk van de REE.

Ook worden de verschillen met de regulatie van glucoseflux, ofwel endogene glucose release, onderstreept. Deze wordt juist voornamelijk bepaald door vetvrije massa (FFM) en, naar nu blijkt, ook door lichaamsvet. Tijdens hyperinsulinemie wordt glucoseflux, in tegenstelling tot vetzuurflux, evident beïnvloed door fitheid $\left(\mathrm{VO}_{2 \max }\right)$, plasma-FFA $(\mu \mathrm{mol} / \mathrm{L})$ en vetpercentage. Kennelijk verloopt de metabole verbetering zoals gezien wordt bij verbeterde fitheid, niet rechtstreeks via de beïnvloeding van vetzuurflux.

Hoofdstuk 3 richt zich op de pathofysiologie van obesitas, waarbij het metabole syndroom en de rol van visceraal vet worden belicht. Hoofdstuk 3.2 beschrijft hoe hormonen de verdeling van brandstofmoleculen (vet, glucose, eiwitten) beïnvloeden en hun lot mede helpen bepalen (verbranding, opslag, gebruik als bouwstof etc). Er wordt specifiek ingegaan op de veranderde spiegels en functies van de betreffende hormonen in obesitas.

Hoofdstuk 4 is volledig gewijd aan de behandeling van abdominale obesitas en de metabole consequenties daarvan. Eerst wordt een overzicht gegeven van de bestaande behandelopties, met speciale aandacht voor Diet/ex en thiazolidinedionetherapie en wat bekend is over beider werkingsmechanismen. De hoofdstukken 4.2 en 4.3 evalueren het gebruik van respectievelijk testosteron en groeihormoon bij de behandeling van centrale obesitas. Geen van beide wordt door ons aangemoedigd, zeker groeihormoon niet, gezien het feit dat noch lichaamscompositie noch metabole parameters hiermee zichtbaar verbeteren. De insulineresistentie, an sich al een probleem bij UBO, verslechtert bovendien.

Hoofdstuk 4.4.3 beschrijft een toevalsbevinding van ons onderzoek. PIO lijkt een verbetering te geven van parameters passend bij non-alcoholische hepatosteatose (NAFLD). Dit is een veel voorkomende leveraandoening bij insulineresistentie, die meestal benigne verloopt maar in sommige gevallen kan degenereren tot steatohepatitis of zelfs tot levercirrose. Bij type-2diabetici leken rosi- en troglitazone een verbetering van de NAFLD te geven. Onze data zijn echter de eerste die suggereren dat dit ook het geval is bij insulineresistentie zonder diabetes. 
Enige maanden na publicatie van onze data verscheen ook histologische onderbouwing hiervan $(383,384)$.

Dit zou betekenen dat een patiëntengroep, die anders niet behandeld zou worden, mogelijk in aanmerking zou kunnen komen voor behandeling met TZD's.

Hoofdstuk 4.5 is gewijd aan de effecten van de twee insulinesensitiserende opties (Diet/ex en PIO). De hiervoor gebruikte methoden worden uitgebreid uiteengezet in Hoofdstuk 4.5.2, dat de basis vormt voor de hoofdstukken 4.5.3- 4.5.6. 40 centraal obese vrijwilligers worden gerandomiseerd tot het krijgen van PIO of Diet/ex gedurende 20 weken, en worden vóór en na deze interventie bestudeerd aan de hand van abdominale CT-scans, DEXA-scanning, nuchter bloedonderzoek, een intraveneuze glucosetolerantietest, een loopbandtest en radioactieve tracerstudies met vet- en spierbiopten. Alle methoden en technieken staan in dit hoofdstuk beschreven.

Hoofdstuk 4.5.3 beschrijft de effecten van PIO op vetzuurkinetiek. Alle TZD's verlagen immers plasma FFA's, maar niet alle TZD's beïnvloeden plasmalipiden zoals TG- en LDLconcentraties. De effecten van PIO op vetzuurflux, die model staat voor lipolyse, worden bestudeerd en afgezet tegen de effecten van Diet/ex om zo onderscheid te kunnen maken tussen de effecten van insulinesensitisatie per se enerzijds en directe PIO-effecten anderzijds.

Zoals bekend dalen FFA-concentraties na PIO-therapie. Dit blijkt te berusten op een verhoging van vetzuurklaring uit de circulatie van ca $40 \%$, niet op een vermindering van de absolute lipolyse. Wel blijkt, zoals verwacht, de lipolyse gevoeliger te zijn voor onderdrukking door insuline: het onveranderde absolute lipolyse niveau post-PIO wordt immers bereikt onder veel lagere insulinespiegels. Na Diet/ex dalen zowel absolute als relatieve lipolyse, maar verandert de vetzuurklaring niet.

In het hoofdstuk wordt gespeculeerd over de organen die verantwoordelijk zouden kunnen zijn voor de verhoogde, overigens niet-oxidatieve, vetzuurklaring.

In Hoofdstuk 4.5.4 wordt de relatie tussen adipokines en insulinesensitiviteit, vetmassa en vetverdeling, en insulinesensitisatie bestudeerd. Adipokines zijn substanties die (o.a.) door vetweefsel geproduceerd worden en een vermeende functie hebben in het mediëren van insulineresistentie; het is echter onduidelijk of zij een centrale rol hierbij spelen en of de eventuele effecten primair gerelateerd zijn aan vet of aan insulinesensitiviteit. Wij hebben gebruik gemaakt van het feit dat wij twee methodes ter beschikking hadden die de insulinegevoeligheid vergrootten, maar tegengestelde effecten hadden op de vetmassa.

Uit onze studies bleken de verschillende geteste adipokines (muv CRP en de cytokines TNF- $\alpha$ en IL-6) echter noch gecorreleerd te zijn met elkaar, noch met insulinegevoeligheid of lichaamscompositie. Alleen adiponectine was invers geassocieerd met insulinegevoeligheid en, bij mannen, ook met vetmassa. Dit bleek bovendien stand te houden na Diet/ex. Het sexeverschil in adiponectine, dat evenals bij CRP reeds bekend was voor baselinedata, bleek ook aanwezig te zijn in de correlaties met insuline-sensitiviteitsparameters en vetverdeling. In de discussie gaan wij nader in op mogelijke verklaringen voor de gevonden geslachtsverschillen en op het feit dat onze data geen ondersteuning bieden voor de hypothese dat adipokines een centrale endocriene rol zouden spelen bij het mediëren van insulineresistentie. Adiponectine vormt, zoals gezegd, hierop een uitzondering.

Post-PIO veranderden adipokinecorrelaties noch concentraties, adiponectine uitgezonderd. Opvallend was de significant sterkere verhoging van adiponectine vergeleken met Diet/ex ondanks maximaal gelijke insulinesensitisatie. Dit lijkt dan ook opnieuw een PIO-specifiek effect te zijn. 
Hoofdstuk 4.5.5 evalueert veranderingen in de grootte en samenstelling van lipoproteinepartikels. Deze zou, naast absolute concentraties van met name LDL en VLDL, een onafhankelijke risicofactor zijn voor het ontwikkelen van hart- en vaatziekten. Dit is echter moeilijk te bestuderen omdat een ongunstig LDL-partikelprofiel (nl kleine LDL-partikels) meestal samenhangt met het metabole syndroom, overgwicht, insulineresistentie en hypertriglyceridemie. Het dalen van plasma TG-spiegels gaat bovendien vaak gepaard met een herverdeling van lipoproteïnecompositie cq het groter worden van LDL-deeltjes.

Aangezien er in onze PIO-groep geen statistisch significante verlaging van TG optrad, maakten we wederom van de gelegenheid gebruik om te bestuderen of en hoe de grootte en samenstelling van LDL- en andere partikels veranderd waren. Deze konden afgezet worden tegen de aspecifieke effecten van insulinesensitisatie, bereikt via Diet/ex. De relatief nieuwe NMR spectroscopie-techniek werd hierbij ingezet.

I.t.t. post-Diet/ex, waren cholesterol en TG concentraties onveranderd na PIO. Na beide behandelingen waren de LDL-partikels echter groter dan voorheen en waren de absolute concentraties van 'large LDL' en 'small LDL' gunstig veranderd. Niettemin waren er duidelijke verschillen tussen PIO en Diet/ex groepen: de PIO- geïnduceerde reshuffling was niet alleen anders verdeeld dan bij de Diet/ex groep (PIO verkleinde bijvoorbeeld ook de VLDLgrootte, echter zonder de intrinsieke verdeling van grootte te beinvloeden) maar was bovendien onafhankelijk van de verandering in Si en van dalingen in TG, totaal of LDL-cholesterol. Bij Diet/ex was er een evidente correlatie met de verandering in Si. Na correctie voor TG bleven deze verschillen bestaan.

Dit impliceert het optreden van een direct effect van PIO op partikelsubklasseverdeling, dat onfhankelijk is van de effecten op insulinesensistisatie.

Hoofdstuk 4.5.6 vergelijkt de effecten van beide interventies op de regionale verdeling en remodellering van vet. PIO bleek de waist-to-hip ratio (WHR) te verminderen, niet, zoals bij Diet/ex het geval was, door een relatief grotere vetmobilisatie uit het buikdepot vergeleken met het femorale vet, maar omgekeerd door het femorale vet te doen toenemen zonder het abdominale vet te veranderen. Veranderingen in de locale vetceldiameter na beide interventies bereikten (net) geen statistische significantie maar ondersteunden de macroscopische verschuivingen in de vetcompartimenten wel.

Deze bevindingen bevestigen dier- en invitrostudies die suggereren dat er regionale verschillen zijn in de gevoeligheid van vetweefsel voor remodellering door TZD's. Ook suggereren onze gegevens dat de verbeterde insulinesensitiviteit door TZD's onafhankelijk zijn van veranderingen in visceraal vet. Hoofdstuk 4.5 .6 bevat daarnaast een kwantitatieve vergelijking tussen PIO en Diet/ex. Hoewel beide vergelijkbare verbeteringen bewerkstelligen van nuchtere glucose, insuline en C-peptideconcentraties, alsmede de glucose $\mathrm{Rd}$ tijdens de clamp, stijgt de insuluinesensitviteits-index Si meer na Diet/ex dan na PIO zonder echter statistische significantie te bereiken. TG, totaal cholesterol en fitheidsparameters verbeteren alleen na Diet/ex. De conclusie is dan ook dat PIO een goede insulinesensitisatie geeft, maar Diet/ex niet kan of zou moeten vervangen.

\section{Beschouwing}

Gezien de complexiteit zal behandeling van obesitas naar verwachting moeilijk blijven, terwijl de behoefte aan behandeling van de daarmee gepaard gaande insulineresistentie toe zal nemen. Dit proefschrift hoopt nieuwe inzichten gegeven te hebben in verscheidene aspecten 
van vetzuurmetabolisme, veranderingen hierin door obesitas en door insulinesensitisatie in het bijzonder. Pioglitazon blijkt meer effecten te bewerkstelligen dan alleen op grond van insulinesensitisatie verklaard of bereikt kan worden. Vele aspecten van de TZD's blijven daarom intrigeren en veel ruimte te bieden voor aanvullend onderzoek. Gezien de laatste inzichten lijken de TZD's veilig toegediend te kunnen worden, alhoewel voorzichtigheid geboden blijft bij hartfalen.

Onzes insziens kunnen zij de additionele positieve effecten van dieet en lichaamsbeweging echter niet evenaren en moeten zij dus niet als substituut gezien worden. 


\section{References}

1. Mokdad AH, Marks JS, Stroup DF, Gerberding JL. Actual causes of death in the United States, 2000. JAMA 2004;291:1238-1245.

2. Flegal KM, Carroll MD, Ogden CL, Johnson CL. Prevalence and trends in obesity among US adults, 1999-2000. JAMA 2002;288:1723-1727.

3. Zimmermann R, Staruss JG, Haemmerle G, Schoiswohl G, Birner-Gruenberger R, Riederer M, Lass A, Neuberger G, Eisenhaber F, Hermetter A, Zechner R. Fat mobilization in adipose tissue is promoted by adipose triglyceride lipase. Science 2004;306:1383-1386.

4. Kershaw EE, Hamm JK, Verhagen LA, Peroni O, Katic M, Flier JS. Adipose triglyceride lipase: function, regulation by insulin, and ccomparison with adiponutrin. Diabetes 2006;55:148-157.

5. Kelley DE, Mandarino LJ. Hyperglycemia Normalizes Insulin-stimulated Skeletal Muscle Glucose Oxidation and Storage in Noninsulin-dependent Diabetes Mellitus. J Clin Invest 1990;86:1999-2007.

6. Frayn KN, Shadid S, Hamlani R, Humphreys SM, Clark ML, Fielding BA, Boland O, Coppack SW. Regulation of fatty acid movement in human adipose tissue in the postabsorptive-to-postprandial transition. Am J Physiol Endocrinol Metab 1994;266:E308-E317.

7. Campbell PJ, Carlson MG, Hill JO, Nurjhan N. Regulation of free fatty acid metabolism by insulin in humans: role of lipolysis and re-esterification. Am J Physiol Endocrinol Metab 1992;263:E1063-E1069.

8. Campbell PJ, Carlson MG, Nurjhan N. Fat metabolism in human obesity. Am J Physiol Endocrinol Metab 1994;266:E600-E605.

9. Evans DJ, Hoffmann RG, Kalkhoff RK, Kissebah AH. Relationship of body fat topography to insulin sensitivity and metabolic profiles in premenopausal women. Metabolism 1984;33:68-75.

10. Barzilai N, She L, Liu B, Vuguin P, Cohen P, Wang J, Rossetti L. Surgical removal of visceral fat reverses hepatic insulin resistance. Diabetes 1999;48:94-98.

11. Evans DJ, Murray R, Kissebah AH. Relationship between skeletal muscle insulin resistance, insulinmediated glucose disposal, and insulin binding. J Clin Invest 1984;74:1515-1525.

12. Coppack SW. Pro-inflammatory cytokines and adipose tissue. Proc Nutr Soc 2001;60:349-356.

13. Festa A, Hanley AJ, Tracy RP, D'Agostino R, Jr., Haffner SM. Inflammation in the prediabetic state is related to increased insulin resistance rather than decreased insulin secretion. Circulation 2003;108:18221830.

14. Ronti T, Lupatteli G, Mannarino E. The endocrine function of adipose tissue: an update. Clin Endocrinol 2006;64:355-365.

15. Vettor R, Milan G, Rossato M, Federsil G. Review article: adipocytokines and insulin resistance. Aliment Pharmacol Ther 2005;22:3-10.

16. Arner P. Regional differences in protein production by human adipose tissue. Biochem Soc Trans 2001;29:72-75

17. Vernon RG, Denis RGP, Sorensen A. Signals of adiposity. Domest Anim Endocrinol 2001;21:197-214.

18. Chicurel M. Whatever happened to leptin? Nature 2000;404:538-540.

19. Hotamisligil GS, Arner P, Caro JF, Atkinson RL, Spiegelman BM. Increased adipose tissue expression of tumor necrosis factor- $\square$ in human obesity and insulin resistance. J Clin Invest 1995;95:2409-2415.

20. Fasshauer M, Klein J, Neumann S, Eszlinger M, Paschke R. Tumor necrosis factor alpha is a negative regulator of resistin gene expression and secretion in 3T3-L1 adipocytes. Biochem Biophys Res Commun 2001;288:1027-1031.

21. Carey AL, Febbraio MA. Interleukin-6 and insulin sensitivity: friend or foe? Diabetologia 2004;47:11351142 .

22. Lyngso D, Simonsen L, Bulow J. Metabolic effects of interleukin-6 in human splanchnic and adipose tissue. J Physiol 2002;543:379-386.

23. McTernan PG, McTernan CL, Chetty R, Jenner K, Fisher FM, Lauer MN, Crocker J, Barnett AH, Kumar $\mathrm{S}$. Increased resistin gene and protein expression in human abdominal adipose tissue. J Clin Endocrinol Metab 2002;87:2407-2410. 
24. Steppan CM, Lazar MA. Resistin and obesity-associated insulin resistance. Trends Endocrinol Metab 2002;13:18-23.

25. Romano M, Guagnano MT, Pacini G, Vigneri S, Falco A, Marinopiccoli M, Manigrasso MR, Basili S, Davi G. Association of inflammation markers with impaired insulin sensitivity and coagulative activation in obese healthy women. J Clin Endocrinol Metab 2003;88:5321-5326.

26. Festa A, D’Agostino R, Jr., Howard G, Mykkänen L, Tracy RP, Haffner SM. Chronic subclinical inflammation as part of the insulin resistance syndrome. The Insulin Resistance Atherosclerosis Study (IRAS). Circulation 2000;102:42-47.

27. Marfella R, Esposito K, Siniscalchi M, Cacciapuoti F, Giugliano F, Labriola D, Ciotola M, Di Palo C, Misso L, Giugliano D. Effect of weight loss on cardiac synchronization and proinflammatory cytokines in premenopausal obese women. Diabetes Care 2004;27:47-52.

28. Haffner SM, Greenberg AS, Weston WM, Chen H, Williams K, Freed MI. Effect of rosiglitazone treatment on nontraditional markers of cardiovascular disease in patients with type 2 diabetes mellitus. Circulation 2002;106:679-684

29. Hak AE, Stehouwer CD, Bots ML, Polderman KH, Schalkwijk CG, Westendorp IC, Hofman A, Witteman JC. Associations of C-reactive protein with measures of obesity, insulin resistance, and subclinical atherosclerosis in healthy, middle-aged women. Arterioscler Thromb Vasc Biol 1999;19:1986-1991.

30. Heilbronn LK, Noakes M, Clifton PM. Energy restriction and weight loss on very-low-fat diets reduce Creactive protein concentrations in obese, healthy women. Arterioscler Thromb Vasc Biol 2001;21:968970.

31. Saijo Y, Kiyota N, Kawasaki Y, Miyazaki T, Kashimura J, Fukuda M, Kishi R. Relationship between Creactive protein and visceral adipose tissue in healthy Japanese subjects. Diabetes Obes Metab 2004;6:249-258.

32. Pieroni L, Bastard JP, Piton A, Khalil L, Hainque B, Jardel C. Interpretation of circulating C-reactive protein levels in adults: body mass index and gender are a must. Diabetes Metab 2003;29:133-138.

33. Diez JJ, Iglesias P. The role of the novel adipocyte-derived hormone adiponectin in human disease. Eur J Endocrinol 2003;148:293-300.

34. Guerre-Millo M. Adipose tissue hormones. J Endocrinol Invest 2002;25:855-861.

35. Schaffler A, Herfarth H, Paul G, Ehling A, Muller-Ladner U, Scholmerich J, Zietz B. Identification of influencing variables on adiponectin serum levels in diabetes mellitus type 1 and type 2. Exp Clin Endocrinol Diabetes 2004;112:383-389.

36. Cnop M, Havel PJ, Utzschneider KM, Carr DB, Sinha MK, Boyko EJ, Retzlaff BM, Knopp RH, Brunzell JD, Kahn SE. Relationship of adiponectin to body fat distribution, insulin sensitivity, and plasma lipoproteins: evidence for independent roles of age and sex. Diabetologia 2003;2003:4-459.

37. Jensen MD, Haymond MW, Rizza RA, Cryer PE, Miles JM. Influence of body fat distribution on free fatty acid metabolism in obesity. J Clin Invest 1989;83:1168-1173.

38. Roust LR, Jensen MD. Postprandial free fatty acid kinetics are abnormal in upper body obesity. Diabetes 1993;42:1567-1573.

39. Unger RH. Lipotoxicity in the pathogenesis of obesity-dependent NIDDM. Diabetes 1995;44:863-869.

40. Richelsen B. Increased alpha 2- but similar beta-adrenergic receptor activities in subcutaneous gluteal adipocytes from females compared with males. Eur J Clin Invest 1986;16:302-309.

41. Raison J, Basdevant A, Sitt Y, Guy-Grand B. Regional differences in adipose tissue lipoprotein lipase activity in relation to body fat distribution and menopausal status in obese women. Int $\mathrm{J}$ Obes 1988;12:465-472.

42. Jensen MD. Gender differences in regional fatty acid metabolism before and after meal ingestion. J Clin Invest 1995;96:2297-2303.

43. Guo ZK, Hensrud DD, Johnson CM, Jensen MD. Regional postprandial fatty acid metabolism in different obesity phenotypes. Diabetes 1999;48:1586-1592.

44. Martin ML, Jensen MD. Effects of body fat distribution on regional lipolysis in obesity. J Clin Invest 1991;88:609-613.

45. Basu A, Basu R, Shah P, Vella A, Rizza RA, Jensen MD. Systemic and regional free fatty acid metabolism in type 2 diabetes. Am J Physiol Endocrinol Metab 2001;280:E1000-E1006.

46. Burguera B, Proctor DN, Dietz W, Guo Z, Joyner MJ, Jensen MD. Leg FFA kinetics during exercise in men and women. Am J Physiol Endocrinol Metab 2000;278:E113-E117.

47. Nielsen S, Guo ZK, Johnson CM, Hensrud DD, Jensen MD. Splanchnic lipolysis in human obesity. J Clin Invest 2004;113:1582-1588. 
48. Rebuffe-Scrive M, Lennart E, Crona N, Smith U, Abrahamsson L, Bjorntorp P. Fat cell metabolism in different regions in women. J Clin Invest 1985;75:1973-1976.

49. Marin P, Rebuffe-Scrive M, Bjorntorp P. Uptake of triglyceride fatty acids in adipose tissue in vivo in man. Eur J Clin Invest 1990;20:158-165.

50. Marin P, Andersson B, Ottosson M, Olbe L, Chowdhury B, Kvist H, Holm G, Sjostrom L, Bjorntorp P. The morphology and metabolism of intraabdominal adipose tissue in men. Metabolism 1992;41:12421248.

51. Marin P, Oden B, Bjorntorp P. Assimilation and mobilization of triglycerides in subcutaneous abdominal and femoral adipose tissue in vivo in men: effects of androgens. J Clin Endocrinol Metab 1995;80:239243.

52. Romanski SA, Nelson R, Jensen MD. Meal fatty acid uptake in adipose tissue: Gender effects in nonobese humans. Am J Physiol Endocrinol Metab 2000;279:E455-E462.

53. Jensen MD, Sarr MG, Dumesic DA, Southorn PA, Levine JA. Regional uptake of meal fatty acids in humans. Am J Physiol Endocrinol Metab 2003;285:E1282-E1288.

54. Uranga AP, Levine J, Jensen M. Isotope tracer measures of meal fatty acid metabolism: reproducibility and effects of the menstrual cycle. Am J Physiol Endocrinol Metab 2005;288:E547-E555.

55. Abbasi F, Chu JW, McLaughlin T, Lamendola C, Leary ET, Reaven GM. Effect of metformin treatment on multiple cardiovascular disease risk factors in patients with type 2 diabetes mellitus. Metabolism 2004;53:159-164.

56. Jensen MD, Rogers PJ, Ellman MG, Miles JM. Choice of infusion-sampling mode for tracer studies of free fatty acid metabolism. Am J Physiol 1988;254:E562-E565.

57. Christie WW. Rapid separation and quantification of lipid classes by high pressure liquid chromatography and mass detection. J Lipid Res 1985;26:507-512.

58. Humphreys SM, Fisher RM, Frayn KN. Micromethod for measurement of sub-nanomole amounts of triacylglycerol. Ann Clin Biochem 1990;27:597-598.

59. Tchoukalova YD, Harteneck DA, Karwoski RA, Tarara J, Jensen MD. A quick, reliable, and automated method for fat cell sizing. J Lipid Res 2003;44:1795-1801.

60. Jensen MD, Kanaley JA, Reed JE, Sheedy PF. Measurement of abdominal and visceral fat with computed tomography and dual-energy x-ray absorptiometry. Am J Clin Nutr 1995;61:274-278.

61. Edens NK, Fried SK, Kral JG, Hirsch J, Leibel RL. In vitro lipid synthesis in human adipose tissue from three abdominal sites. Am J Physiol 1993;265:E374-E379.

62. Patterson BW, Mittendorfer B, Elias N, Satyanarayana R, Klein S. Use of stable isotopically labeled tracers to measure very low density lipoprotein-triglyceride turnover. J Lipid Res 2002;43:223-233.

63. Meek S, Nair KS, Jensen MD. Insulin regulation of regional free fatty acid metabolism. Diabetes 1999;48:10-14.

64. Lillioja S, Foley J, Bogardus C, Mott D, Howard BV. Free fatty acid metabolism and obesity in man: in vivo in vitro comparisons. Metabolism 1986;35:505-514.

65. Ferrannini E, Barrett EJ, Bevilacqva S, DeFronzo RA. Effect of fatty acids on glucose production and utilization in man. J Clin Invest 1983;72:1737-1747.

66. Nielsen S, Guo Z, Albu JB, Klein S, O’Brien PC, Jensen MD. Energy expenditure, sex, and endogenous fuel availability in humans. J Clin Invest 2003;111:981-988.

67. Gastaldelli A, Miyazaki Y, Pettiti M, Buzzigoli E, Mahankali S, Ferrannini E, DeFronzo RA. Separate contribution of diabetes, total fat mass, and fat topography to glucose production, gluconeogenesis, and glycogenolysis. J Clin Endocrinol Metab 2004;89:3914-3921.

68. Gastaldelli A, Miyazaki Y, Pattiti M, Matsuda M, Mahankali S, Santini E, DeFronzo RA, Ferrannini E. Metabolic effects of visceral fat accumulation in type 2 diabetes. J Clin Endocrinol Metab 2002;87:50985103.

69. Groop LC, Bonadonna RC, DelPrato S, Ratheiser K, Zyck K, Ferrannini E, DeFronzo RA. Glucose and free fatty acid metabolism in non-insulin-dependent diabetes mellitus. J Clin Invest 1989;84:205-213.

70. Dengel DR, Pratley RE, Hagberg JM, Rogus EM, Goldberg AP. Distinct effects of aerobic exercise training and weight loss on glucose homeostasis in obese sedentary men. J Appl Physiol 1996;81:318-325.

71. Nielsen S, Halliwill JR, Joyner MJ, Jensen MD. Vascular response to angiotensin II in upper body obesity. Hypertension 2004;44:435-441.

72. Doan AE, Peterson DR, Blackmon JR, Bruce RA. Myocardial ischemia after maximal exercise in healthy men. Am Heart J 1965;69:11-25. 
73. Kury D, Keller U. Trimethylsilyl-O-methyloxime derivatives for the measurement of [6,6-2H2]-Dglucose-enriched plasma samples by gas chromatography-mass spectrometry. J Chrom 1991;572:302306.

74. Causon RC, Carruthers ME, Rodnight R. Assay of plasma catecholamines by liquid chromatography with electrochemical detection. Anal Biochem 1981;116:223-226.

75. Miles JM, Ellman MG, McClean KL, Jensen MD. Validation of a new method for determination of free fatty acid turnover. Am J Physiol Endocrinol Metab 1987;252:E431-E438.

76. Gastaldelli A, Baldi S, Pettiti M, Toschi E, Camastra S, Natali A, Landau BR, Ferrannini E. Influence of obesity and type 2 diabetes on gluconeogenesis and glucose output in humans: a quantitative study. Diabetes 2000;49:1367-1373.

77. Tanner JM. Fallacy of per-weight and per-surface area standards, and their relation to spurious correlation. J Appl Physiol 1949;2:1-15.

78. Bogardus C, Lillioja S, Lukuki Nyomba B, Zurlo F, Swinburn B, Esposito-Del Puente A, Knowler WC, Ravussin E, Mott DM, Bennett PH. Distribution of in vivo insulin action in Pima Indians as mixture of three normal distributions. Diabetes 1989;38:1423-1431.

79. Bogardus C, Lillioja S, Mott DM, Hollenbeck CB, Reaven GM. Relationship between degree of obesity and in vivo insulin action in man. American Journal of Physiology 1985;248:E286-E291.

80. DeFronzo RA, Sherwin RS, Kraemer N. Effect of physical training on insulin action in obesity. Diabetes 1987;36:1379-1385.

81. Michaud DS, Giovannucci E, Willett WC, Colditz GA, Stampfer MJ, Fuchs CS. Physical activity, obesity, height, and the risk of pancreatic cancer. JAMA 2001;286:921-929.

82. Jee SH, Ohrr H, Sull JW, Yun JE, Ji M, Samet JM. Fasting serum glucose level and cancer risk in Korean men and women. JAMA 2005;293:194-202.

83. Lorincz AM, Sukumar S. Molecular links between obesity and breast cancer. Endocr Relat Cancer 2006;13:279-292.

84. Xu WH, Xiang YB, Zheng W, Zhang X, Ruan ZX, Cheng JR, Gao YT, Shu XO. Weight history and risk of endometrial cancer among Chinese women. Int J Epidemiol 2006;35:159-166.

85. Obesity: Clinical Evaluation and Treatment. Eckel, R. H. 2003. Lippincott, Williams and Wilkins. Ref Type: Serial (Book,Monograph)

86. Scacchi M, Pincelli AI, Cavagnini F. Growth hormone in obesity. Int J Obes Relat Metab Disord 1999;23:260-271.

87. Andrew R, Phillips DI, Walker BR. Influence of gender and body composition on glucocorticoid metabolism in middle-aged humans. Int J Obes Relat Metab Disord 2000;24 Suppl 2:S144-S145.

88. Björntorp P, Rosmond R. Obesity and cortisol. Nutrition 2000;16:924-936.

89. Pasquali R, Vicennati V. Activity of the hypothalamic-pituitary-adrenal axis in different obesity phenotypes. Int J Obes Relat Metab Disord 2000;24 Suppl 2:S47-S49.

90. Levine JA. Relation between chubby cheeks and visceral fat. N Engl J Med 1998;339:1946-1947.

91. Couillard C, Gagnon J, Bergeron J, Leon AS, Rao DC, Skinner JS, Wilmore JH, Despres JP, Bouchard C. Contribution of body fatness and adipose tissue distribution to the age variation in plasma steroid hormone concentrations in men: the HERITAGE Family Study. J Clin Endocrinol Metab 2000;85:10261031.

92. Jensen MD. Androgen effect on body composition and fat metabolism. Mayo Clin Proc 2000;75:S65S69.

93. Ford ES, Giles WH, Mokdad AH. Increasing prevalence of the metabolic syndrome among U.S. adults. Diabetes Care 2004;24:2444-2449.

94. Lim S, Jang HC, Lee HK, Kimm KC, Park C, Cho NH. A rural-urban comparison of the characteristics of the metabolic syndrome by gender in Korea: The Korean Health and Genome Study (KHGS). J Endocrinol Invest 2006;29:313-319.

95. Deepa M, Farooq S, Datta M, Deepa R, Mohan V. Prevalence of metabolic syndrome using WHO, ATPIII, and IDF definitions in Asian Indians: the Chennai Urban Rural Epidemiology Study (CURES-34). Diabetes Metab Res Rev 2006; [EPub ahead of print]:

96. Maggi S, Noale M, Gallina P, Bianchi D, Marzari C, Limongi F, Crepaldi G. Metabolic syndrome, diabetes, and cardiovascular disease in an elderly Caucasian cohort: the Italian longitudinal study on aging. J Gerontol A Biol Sci Med Sci 2006;61:505-510.

97. Roden M, Price TB, Perseghin G, Petersen KF, Rothman DL, Cline GW, Shulman GI. Mechanism of free fatty acid-induced insulin resistance in humans. J Clin Invest 1996;97:2859-2865. 
98. Bergman RN, Van Citters GW, Mittelman SD, Dea MK, Hamilton-Wessler M, Kim SP, Ellmerer M. Central role of the adipocyte in the metabolic syndrome. J Investig Med 2001;49:119-126.

99. Mittelman SD, Fu YY, Rebrin K, Steil G, Bergman RN. Indirect effect of insulin to suppress endogenous glucose production is dominant, even with hyperglucagonemia. J Clin Invest 1997;100:3121-3130.

100. Tchernof A, Belanger C, Morisset AS, Richard C, Mailloux J, Laberge P, Dupont P. Regional differences in adipose tissue metabolism in women: minor effect of obesity and body fat distribution. Diabetes 2006;55:1353-1360.

101. Boden G. Role of fatty acids in the pathogenesis of insulin resistance and NIDDM. Diabetes 1997;46:310 .

102. Kim SP, Ellmerer M, Van Citters GW, Bergman RN. Primacy of hepatic insulin resistance in the development of the metabolic syndrome induced by an isocaloric moderate-fat diet in the dog. Diabetes 2003;52:2453-2460.

103. Bains RK, Wells SE, Flavell DM, Fairhall KM, Stom M, Le Tissier P, Robinson IC. Visceral obesity without insulin resistance in late-onset obesity rats. Endocrinology 2004;145:2666-2679.

104. Flatt JP. Carbohydrate balance and food intake regulation. Am J Clin Nutr 1995;62:155-157.

105. Lafontan M, Berlan M. Fat cell $\square_{2}$-adrenoceptors: the regulation of fat cell function and lipolysis. Endocr Rev 1995;16:716-738.

106. Acheson KJ, Ravussin E, Wahren J, Jequier E. Thermic effect of glucose in man. Obligatory and facultative thermogenesis. J Clin Invest 1984;74:1572-1580.

107. Morgan JB, York DA, Wilkin TJ. Influence of propranolol on the acute thermic effect of feeding in man. Ann Nutr Metab 1986;30:386-392.

108. Segal KR, Albu J, Chun A, Edano A, Legaspi B, Pi-Sunyer FX. Independent effects of obesity and insulin resistance on postprandial thermogenesis in men. J Clin Invest 1992;89:824-833.

109. Saloranta C, Franssila-Kallunki A, Ekstrand A, Taskinen MR, Groop L. Modulation of hepatic glucose production by non-esterified fatty acids in type 2 (non-insulin-dependent) diabetes mellitus. Diabetologia 1991;34:409-415.

110. Kelley DE, Mokan M, Simoneau JA, Mandarino LJ. Interaction between glucose and free fatty acid metabolism in human skeletal muscle. J Clin Invest 1993;92:91-98.

111. Rigalleau V, Binnert C, Minehira K, Stefanoni N, Schneiter P, Henchoz E, Matzinger O, Cayeux C, Jéquier E, Tappy L. In normal men, free fatty acids reduce peripheral but not splanchnic glucose uptake. Diabetes 2001;50:727-732.

112. Jensen MD, Haymond MW, Gerich JE, Cryer PE, Miles JM. Lipolysis during fasting. Decreased suppression by insulin and increased stimulation by epinephrine. J Clin Invest 1987;79:207-213.

113. Jensen MD, Miles JM, Gerich JE, Cryer PE, Haymond MW. Preservation of insulin effects on glucose production and proteolysis during fasting. Am J Physiol 1988;254:E700-7.

114. Jensen MD, Haymond MW. Protein metabolism in obesity: effects of body fat distribution and hyperinsulinemia on leucine turnover. Am J Clin Nutr 1991;53:172-176.

115. Meek S, Persson M, Ford GC, Nair KS. Differential regulation of amino acid exchange and protein dynamics across splanchnic and skeletal muscle beds by insulin in healthy human subjects. Diabetes 1998;47:1824-1835.

116. Eckel RH. Insulin resistance: an adaptation for weight maintenance. Lancet 1992;340:1452-1453.

117. Richelsen B, Pedersen SB, Kristensen K, Borglum JD, Norrelund H, Christiansen JS, Jorgensen JO. Regulation of lipoprotein lipase and hormone-sensitive lipase activity and gene expression in adipose and muscle tissue by growth hormone treatment during weight loss in obese patients. Metabolism 2000;49:906-911.

118. Bengtsson B-A, Brummer R-JM, Edén S, Sjöström L. Effects of growth hormone on fat mass and fat distribution. Acta Paediatr 1992;383:62-65.

119. Mauras N, O'Brien KO, Welch S, Rini A, Helgeson K, Vieira NE, Yergey AL. Insulin-like growth factor I and growth hormone $(\mathrm{GH})$ treatment in $\mathrm{GH}$ - deficient humans: differential effects on protein, glucose, lipid, and calcium metabolism. J Clin Endocrinol Metab 2000;85:1686-1694.

120. Slavin BG, Ong JM, Kern PA. Hormonal regulation of hormone-sensitive lipase activity and mRNA levels in isolated rat adipocytes. J Lipid Res 1994;35:1535-1541.

121. Biller BM, Sesmilo G, Baum HB, Hayden D, Schoenfeld D, Klibanski A. Withdrawal of long-term physiological growth hormone $(\mathrm{GH})$ administration: differential effects on bone density and body composition in men with adult-onset GH deficiency. J Clin Endocrinol Metab 2000;85:970-976.

122. Brummer R-JM. Effects of growth hormone treatment on visceral adipose tissue. Growth Horm IGF Res 1998;8:19-23. 
123. Russell-Jones DL, Bowes SB, Rees SE, Jackson NC, Weissberger AJ, Hovorka R, Sonksen PH, Umpleby AM. Effect of growth hormone treatment on postprandial protein metabolism in growth hormonedeficient adults. Am J Physiol 1998;274:E1050-E1056.

124. O’Sullivan AJ, Kelly JJ, Hoffman DM, Freund J, Ho KKY. Body composition and energy expenditure in acromegaly. J Clin Endocrinol Metab 1994;78:381-386.

125. Di Girolamo M, Eden S, Enberg G, Isaksson O, Lonnroth P, Hall K, Smith U. Specific binding of human growth hormone but not insulin-like growth factors by human adipocytes. FEBS Lett 1986;205:15-19.

126. O’Sullivan AJ, Kelly JJ, Hoffman DM, Baxter RC, Ho KK. Energy metabolism and substrate oxidation in acromegaly. J Clin Endocrinol Metab 1995;80:486-491.

127. Hoffman DM, O’Sullivan AJ, Freund J, Ho KK. Adults with growth hormone deficiency have abnormal body composition but normal energy metabolism. J Clin Endocrinol Metab 1995;80:72-77.

128. Salomon F, Cuneo RC, Hesp R, Sonksen PH. The effects of treatment with recombinant human growth hormone on body composition and metabolism in adults with growth hormone deficiency. $\mathrm{N}$ Engl $\mathrm{J}$ Med 1989;321:1797-1803.

129. Karlsson C, Stenlof K, Johannsson G, Mårin P, Björntorp P, Bengtsson B-A, Carlsson B, Carlsson LMS, Sjöström L. Effects of growth hormone treatment on the leptin system and on energy expenditure in abdominally obese men. Eur J Endocrinol 1998;138:408-414.

130. Vaisman N, Zadik Z, Akivias A, Voet H, Katz I, Yair S, Ashkenazi A. Changes in body composition, resting energy expenditure, and thermic effect of food in short children on growth hormone therapy. Metabolism 1994;43:1543-1548.

131. Bjorgell P, Rosberg S, Isaksson O, Belfrage P. The antilipolytic, insulin-like effect of growth hormone is caused by a net decrease of hormone-sensitive lipase phosphorylation. Endocrinology 1984;115:11511156.

132. Oscarsson J, Ottosson M, Vikman-Adolfsson K, Frick F, Enerback S, Lithell H, Eden S. GH but not IGFI or insulin increases lipoprotein lipase activity in muscle tissues of hypophysectomised rats. J Endocrinol 1999;160:247-255.

133. Ottosson M, Vikman-Adolfsson K, Enerback S, Elander A, Björntorp P, Eden S. Growth hormone inhibits lipoprotein lipase activity in human adipose tissue. J Clin Endocrinol Metab 1995;80:936-941.

134. Richelsen B. Effect of growth hormone on adipose tissue and skeletal muscle lipoprotein lipase activity in humans. J Endocrinol Invest 1999;22:10-15.

135. Guyton AC, Hall JE: Textbook of medical Physiology. 2000,

136. Goodman HM. Effects of growth hormone on glucose utilization in diaphragm muscle in the absence of increased lipolysis. Endocrinology 1967;81:1099-1103.

137. Seng G, Galgoti C, Louisy P, Toussain P, Drouin P, Debry G. Metabolic effects of a single administration of growth hormone on lipid and carbohydrate metabolism in normal-weight and obese subjects. Am J Clin Nutr 1989;50:1348-1354.

138. Umpleby AM, Russell-Jones DL. The hormonal control of protein metabolism. Baillieres Clin Endocrinol Metab 1996;10:551-570.

139. Garlick PJ, McNurlan MA, Bark T, Lang CH, Gelato MC. Hormonal regulation of protein metabolism in relation to nutrition and disease. J Nutr 1998;128:356S-359S.

140. Moller N, Jorgensen JO, Alberti KG, Flyvbjerg A, Schmitz O. Short-term effects of growth hormone on fuel oxidation and regional substrate metabolism in normal man. J Clin Endocrinol Metab 1990;70:11791186.

141. Fryburg DA, Barrett EJ. Growth hormone acutely stimulates skeletal muscle but not whole-body protein synthesis in humans. Metabolism 1993;42:1223-1227.

142. Lucidi P, Lauteri M, Laureti S, Celleno R, Santoni S, Volpi E, Angeletti G, Santeusanio F, De Feo P. A dose-response study of growth hormone $(\mathrm{GH})$ replacement on whole body protein and lipid kinetics in GH-deficient adults. J Clin Endocrinol Metab 1998;83:353-357.

143. Maccario M, Grottoli S, Procopio M, Oleandri SE, Rossetto R, Gauna C, Arvat E, Ghigo E. The GH/IGF$\mathrm{I}$ axis in obesity: Influence of neuro-endocrine and metabolic factors. Int $\mathrm{J}$ Obes Relat Metab Disord 2000;24:S96-S99.

144. Richelsen B, Pedersen SB, Børglum JD, Møller-Pedersen T, Jørgensen J, Jørgensen JO. Growth hormone treatment of obese women for $5 \mathrm{wk}$ : effect on body composition and adipose tissue LPL activity. Am J Physiol 1994;266:E211-E216.

145. Tagliaferri M, Scacchi M, Pincelli AI, Berselli ME, Silvestri P, Montesano A, Ortolani S, Dubini A, Cavagnini F. Metabolic effects of biosynthetic growth hormone treatment in severely energy-restricted obese women. Int J Obes 1998;22:836-841. 
146. Snyder DK, Clemmons DR, Underwood LE. Treatment of obese, diet-restricted subjects with growth hormone for 11 weeks: Effects on anabolism, lipolysis, and body composition. J Clin Endocrinol Metab 1988;67:54-61.

147. Snyder DK, Underwood LE, Clemmons DR. Persistent lipolytic effect of exogenous growth hormone during caloric restriction. Am J Med 1995;98:129-134.

148. Drent ML, Wever LDV, Adèr HJ, van der Veen EA. Growth hormone administration in addition to a very low calorie diet and an exercise program in obese subjects. Eur J Endocrinol 1995;132:565-572.

149. Snyder DK, Underwood LE, Clemmons DR. Anabolic effects of growth hormone in obese diet-restricted subjects are dose dependent. Am J Clin Nutr 1990;52:431-437.

150. Skaggs SR, Crist DM. Exogenous human growth hormone reduces body fat in obese women. Horm Res 1991;35:19-24.

151. Kim KR, Nam SY, Song YD, Lim SK, Lee HC, Huh KB. Low-dose growth hormone treatment with diet restriction accelerates body fat loss, exerts anabolic effect and improves growth hormone secretory dysfunction in obese adults. Horm Res 1999;51:78-84.

152. Johannsson G, Mårin P, Lönn L, Ottosson M, Stenlöf K, Björntorp P, Sjöström L, Bengtsson B-A. Growth hormone treatment of abdominally obese men reduces abdominal fat mass, improves glucose and lipoprotein metabolism, and reduces diastolic blood pressure. J Clin Endocrinol Metab 1997;82:727-734.

153. Thompson JL, Butterfield GE, Gylfadottir UK, Yesavage J, Marcus R, Hintz RL, Pearman A, Hoffman AR. Effects of human growth hormone, insulin-like growth factor I, and diet and exercise on body composition of obese postmenopausal women. J Clin Endocrinol Metab 1998;83:1477-1484.

154. Mauras N, Hayes V, Welch S, Rini A, Helgeson K, Dokler M, Veldhuis JD, Urban RJ. Testosterone deficiency in young men: marked alterations in whole body protein kinetics, strength, and adiposity. J Clin Endocrinol Metab 1998;83:1886-1892.

155. Welle S, Jozefowicz R, Forbes G, Griggs RC. Effect of testosterone on metabolic rate and body composition in normal men and men with muscular dystrophy. J Clin Endocrinol Metab 1992;74:332-335.

156. Arslanian S, Suprasongsin C. Testosterone treatment in adolescents with delayed puberty: changes in body composition, protein, fat, and glucose metabolism. J Clin Endocrinol Metab 1997;82:3213-3220.

157. Buchter D, Behre HM, Kliesch S, Chirazi A, Nieschlag E, Assmann G, von Eckardstein A. Effects of testosterone suppression in young men by the gonadotropin releasing hormone antagonist cetrorelix on plasma lipids, lipolytic enzymes, lipid transfer proteins, insulin, and leptin. Exp Clin Endocrinol Diabetes 1999; 107:522-529.

158. Ferrando AA, Tipton KD, Doyle D, Phillips SM, Cortiella J, Wolfe RR. Testosterone injection stimulates net protein synthesis but not tissue amino acid transport. Am J Physiol Endocrinol Metab 1998;275:E864E871.

159. Xu X, De Pergola G, Bjorntorp P. The effects of androgens on the regulation of lipolysis in adipose precursor cells. Endocrinology 1990;126:1229-1234.

160. Rebuffe-Scrive M, Marin P, Bjorntorp P. Effect of testosterone on abdominal adipose tissue in men. Int $\mathrm{J}$ Obes 1991;15:791-795.

161. Mårin P, Holmäng S, Jönsson L, Sjöström L, Kvist H, Holm G, Lindstedt G, Björntorp P. The effects of testosterone treatment on body composition and metabolism in middle-aged obese men. Int $\mathrm{J}$ Obes 1992;16:991-997.

162. Bhasin S, Storer TW, Berman N, Yarasheski KE, Clevenger B, Phillips J, Lee WP, Bunnell TJ, Casaburi R. Testosterone replacement increases fat-free mass and muscle size in hypogonadal men. J Clin Endocrinol Metab 1997;82:407-413.

163. Seidell JC, Bjorntorp P, Sjostrom L, Kvist H, Sannerstedt R. Visceral fat accumulation in men is positively associated with insulin, glucose, and C-peptide levels, but negatively with testosterone levels. Metabolism 1990;39:897-901.

164. Marin P, Oden B, Olbe L, Bengtsson B-A, Bjorntorp P. Assimilation of triglycerides in subcutaneous and intraabdominal adipose tissues in vivo in men: effects of testosterone. J Clin Endocrinol Metab 1996;81:1018-1022.

165. Ramirez ME, McMurry MP, Wiebke GA, Felten KJ, Ren K, Meikle AW, Iverius PH. Evidence for sex steroid inhibition of lipoprotein lipase in men: comparison of abdominal and femoral adipose tissue. Metabolism 1997;46:179-185.

166. Bjorntorp P. Hormonal regulation of visceral adipose tissue. Growth Horm IGF Res 1998;8 Suppl B:1517. 
167. Lima N, Cavaliere H, Knobel M, Halpern A, Medeiros-Neto G. Decreased androgen levels in massively obese men may be associated with impaired function of the gonadostat. Int J Obes Relat Metab Disord 2000;24:1433-1437.

168. Kraemer WJ, Volek JS, Clark KL, Gordon SE, Puhl SM, Koziris LP, McBride JM, Triplett-McBride NT, Putukian M, Newton RU, Hakkinen K, Bush JA, Sebastianelli WJ. Influence of exercise training on physiological and performance changes with weight loss in men. Med Sci Sports Exerc 1999;31:13201329.

169. Turcato E, Zamboni M, De Pergola G, Armellini F, Zivelonghi A, Bergamo-Andreis IA, Giorgino R, Bosello O. Interrelationships between weight loss, body fat distribution and sex hormones in pre- and postmenopausal obese women. J Intern Med 1997;241:363-372.

170. Wabitsch M, Hauner H, Heinze E, Bockmann A, Benz R, Mayer H, Teller W. Body fat distribution and steroid hormone concentrations in obese adolescent girls before and after weight reduction. J Clin Endocrinol Metab 1995;80:3469-3475.

171. Isidori AM, Caprio M, Strollo F, Moretti C, Frajese G, Isidori A, Fabbri A. Leptin and androgens in male obesity: evidence for leptin contribution to reduced androgen levels. J Clin Endocrinol Metab 1999;84:3673-3680.

172. Vermeulen A, Goemaere S, Kaufman JM. Testosterone, body composition and aging. J Endocrinol Invest 1999;22:110-116.

173. O'Sullivan AJ, Crampton LJ, Freund J, Ho KKY. The route of estrogen replacement therapy confers divergent effects on substrate oxidation and body composition in postmenopausal women. J Clin Invest 1998;102:1035-1040.

174. O'Sullivan AJ, Ho KK. A comparison of the effects of oral and transdermal estrogen replacement on insulin sensitivity in postmenopausal women. J Clin Endocrinol Metab 1995;80:1783-1788.

175. Poehlman ET, Toth MJ, Gardner AW. Changes in energy balance and body composition at menopause: A controlled longitudinal study. Ann Intern Med 1995;123:673-675.

176. Paolisso G, Rizzo MR, Mazziotti G, Rotondi M, Tagliamonte MR, Varricchio G, Carella C, Varricchio M. Lack of association between changes in plasma leptin concentration and in food intake during the menstrual cycle. Eur J Clin Invest 1999;29:490-495.

177. Van Gaal LF, Vanuytsel JL, Vansant GA, De Leeuw IH. Sex hormones, body fat distribution, resting metabolic rate and glucose-induced thermogenesis in premenopausal obese women. Int $\mathrm{J}$ Obes 1994;18:333-338.

178. Piers LS, Diggavi SN, Rijskamp J, van Raajj JMA, Shetty PS, Hautvast JGAJ. Resting metabolic rate and thermic effect of a meal in the follicular and luteal phases of the menstrual cycle in well-nourished Indian women. Am J Clin Nutr 1995;61:296-302.

179. Jensen J, Christiansen C, Rodbro P. Oestrogen-progestogen replacement therapy changes body composition in early post-menopausal women. Maturitas 1986;8:209-216.

180. Toth MJ, Poehlman ET, Matthews DE, Tchernof A, MacCoss MJ. Effects of estradiol and progesterone on body composition, protein synthesis, and lipoprotein lipase in rats. Am J Physiol Endocrinol Metab 2001;280:E496-E501

181. Mauras N. Estrogens do not affect whole-body protein metabolism in the prepubertal female. J Clin Endocrinol Metab 1995;80:2842-2845.

182. Mauras N, O’Brien KO, Klein KO, Hayes V. Estrogen suppression in males: metabolic effects. J Clin Endocrinol Metab 2000;85:2370-2377.

183. Mauriege P, Imbeault P, Prud'Homme D, Tremblay A, Nadeau A, Despres JP. Subcutaneous adipose tissue metabolism at menopause: importance of body fatness and regional fat distribution. J Clin Endocrinol Metab 2000;85:2446-2454.

184. Jensen MD, Martin ML, Cryer PE, Roust LR. Effects of estrogen on free fatty acid metabolism in humans. Am J Physiol 1994;266:E914-20.

185. Pansini F, Bonaccorsi G, Genovesi F, Folegatti MR, Bagni B, Bergamini CM, Mollica G. Influence of estrogens on serum free fatty acid levels in women. J Clin Endocrinol Metab 1990;71:1387-1389.

186. Toth MJ, Sites CK, Eltabbakh GH, Poehlman ET. Effect of menopausal status on insulin-stimulated glucose disposal: comparison of middle-aged premenopausal and early postmenopausal women. Diabetes Care 2000;23:801-806.

187. Tchernof A, Calles-Escandon J, Sites CK, Poehlman ET. Menopause, central body fatness, and insulin resistance: effects of hormone-replacement therapy. Coron Artery Dis 1998;9:503-511.

188. Bjorkelund C, Lissner L, Andersson S, Lapidus L, Bengtsson C. Reproductive history in relation to relative weight and fat distribution. Int J Obes Relat Metab Disord 1996;20:213-219. 
189. Hassager C, Christiansen C. Estrogen/gestagen therapy changes soft tissue body composition in postmenopausal women. Metabolism 1989;38:662-665.

190. Iverius PH, Brunzell JD. Relationship between lipoprotein lipase activity and plasma sex steroid level in obese women. J Clin Invest 1988;82:1106-1112.

191. Norman RJ, Flight IHK, Rees MCP. Oestrogen and progestogen hormone replacement therapy for perimenopausal and post-menopausal women: weight and body fat distribution. The Cochrane Library 2000;

192. Brussaard HE, Leuven JAG, Frölich M, Kluft C, Krans HMJ. Short-term oestrogen replacement therapy improves insulin resistance, lipids and fibrinolysis in postmenopausal women wtih NIDDM. Diabetologia 1997;40:843-849.

193. Brillon DJ, Zheng B, Campbell RG, Matthews DE. Effect of cortisol on energy expenditure and amino acid metabolism in humans. Am J Physiol Endocrinol Metab 1995;268:E501-E513.

194. Putignano P, Kaltsas GA, Korbonits M, Jenkins PJ, Monson JP, Besser GM, Grossman AB. Alterations in serum protein levels in patients wtih Cushing's Syndrome before and after successful treatment. J Clin Endocrinol Metab 2000;85:3309-3312.

195. De Feo P. Hormonal regulation of human protein metabolism. Eur J Endocrinol 1996;135:7-18.

196. Bjorntorp P, Rosmond R. Neuroendocrine abnormalities in visceral obesity. Int J Obes Relat Metab Disord 2000;24 Suppl 2:S80-S85.

197. Castonguay TW. Glucocorticoids as modulators in the control of feeding. Brain Res Bulletin 1991;27:423-428.

198. Richard D, Huang Q, Timofeeva E. The corticotropin-releasing hormone system in the regulation of energy balance in obesity. Int J Obes Relat Metab Disord 2000;24 Suppl 2:S36-S39.

199. Rebuffe-Scrive M, Krotkiewski M, Elfverson J, Björntorp P. Muscle and adipose tissue morphology and metabolism in Cushing's Syndrome. J Clin Endocrinol Metab 1988;67:1122-1128.

200. Shamoon H, Soman V, Sherwin RS. The influence of acute physiological increments of cortisol on fuel metabolism and insulin binding to monocytes in normal humans. J Clin Endocrinol Metab 1980;50:495501.

201. Horber FF, Haymond MW. Human growth hormone prevents the protein catabolic side effects of prednisone in humans. J Clin Invest 1990;86:265-272.

202. Haymond MW, Horber FF. The effects of human growth hormone and prednisone on whole body estimates of protein metabolism. Horm Res 1992;38:44-46.

203. Ottosson M, Lönnroth P, Björntorp P, Edén S. Effects of cortisol and growth hormone on lipolysis in human adipose tissue. J Clin Endocrinol Metab 2000;85:799-803.

204. Slavin BG, Ong JM, Kern PA. Hormonal regulation of hormone-sensitive lipase activity and mRNA levels in isolated rat adipocytes. J Lipid Res 1994;35:1535-1541.

205. Lönn L, Kvist H, Sjostrom L. Changes in body composition and adipose tissue distribution after treatment of women with Cushing's syndrome. Metabolism 1994;43:1517-1522.

206. Ottosson M, Vikman-Adolfsson K, Enerbäck S, Olivecrona G, Björntorp P. The effects of cortisol on the regulation of lipoprotein lipase activity in human adipose tissue. J Clin Endocrinol Metab 1994;79:820825 .

207. Krotkiewski M, Bjorntorp P, Smith U. The effect of long-term dexamethasone treatment on lipoprotein lipase activity in rat fat cells. Horm Metab Res 1976;8:245-246.

208. Gelfand RA, Matthews DE, Bier DM, Sherwin RS. Role of counterregulatory hormones in the catabolic response to stress. J Clin Invest 1984;74:2238-2248.

209. De Feo P, Perriello G, Torlone E, Ventura MM, Fanelli C, Santeusanio F, Brunetti P, Gerich JE, Bolli GB. Contribution of cortisol to glucose conterregulation in humans. Am J Physiol Endocrinol Metab 1989;257:E35-E42.

210. Buemann B, Vohl MC, Chagnon M, Chagnon YC, Gagnon J, Perusse L, Dionne F, Despres JP, Tremblay A, Nadeau A, Bouchard C. Abdominal visceral fat is associated with a BclI restriction fragment length polymorphism at the glucocorticoid receptor gene locus. Obes Res 1997;5:186-192.

211. Reid IR, Wattie DJ, Evans MC, Stapleton JP. Testosterone therapy in glucocorticoid-treated men. Arch Intern Med 1996;156:1173-1177.

212. Scheen AJ, Finer N, Hollander P, Jensen MD, Van Gaal LF, RIO-Diabetes Study Group. Efficacy and tolerability of rimonabant in overweight or obese patients with type 2 diabetes: a randomised controlled study. Lancet 2006;368:1660-1672.

213. Mårin P, Krotkiewski M, Björntorp P. Androgen treatment of middle-aged, obese men: effects on metabolism, muscle and adipose tissues. Eur J Med 1992;1:329-336. 
214. Mårin P, Holmäng S, Gustafsson C, Jönsson L, Kvist H, Elander A, Eldh J, Sjöström L, Holm G, Björntorp P. Androgen treatment of abdominally obese men. Obes Res 1993;1:245-251.

215. Dumesic DA, Abbott DH, Eisner JR, Herrmann RR, Reed JE, Welch TJ, Jensen MD. Pituitary desensitization to gonadotropin releasing hormone increases abdominal adiposity in hyperadrenogenic anovulatory women. Fertil Steril 1998;70:94-101.

216. Matsumoto AM, Sandblom RE, Schoene RB, Lee KA, Giblin EC, Pierson DJ, Bremner WJ. Testosterone replacement in hypogonadal men: effects on obstructive sleep apnea, respiratory drives, and sleep. Clin Endocrinol (Oxf) 1985;22:713-721.

217. Schneider BK, Pickett CK, Zwillich CW, Weil JV, McDermott MT, Santen RJ, Varano LA, White DP. Influence of testosterone on breathing during sleep. J Appl Physiol 1986;61:618-623.

218. Münzer T, Harman SM, Hees P, Shapiro E, Christmas C, Bellantoni MF, Stevens TE, O'Connor KG, Pabst KM, St.Clair C, Sorkin JD, Blackman MR. Effects of GH and/or sex steroid administration on abdominal subcutaneous and visceral fat in healthy aged women and men. J Clin Endocrinol Metab $2001 ; 86: 3604-3610$

219. Norrelund H, Borglum J, Jorgensen JO, Richelsen B, Moller N, Sreekumaran NK, Christiansen JS. Effects of growth hormone administration on protein dynamics and substrate metabolism during 4 weeks of dietary restriction in obese women. Clin Endocrinol (Oxf) 2000;52:305-312.

220. Iranmanesh A, Lizarralde G, Veldhuis JD. Age and relative adiposity are specific negative determinants of the frequency and amplitude of growth hormone $(\mathrm{GH})$ secretory bursts and the half-life of endogenous GH in healthy men. J Clin Endocrinol Metab 1991;73:1081-1088.

221. Veldhuis JD, Iranmanesh A, Ho KK, Waters MJ, Johnson ML, Lizarralde G. Dual defects in pulsatile growth hormone secretion and clearance subserve the hyposomatotropism of obesity in man. J Clin Endocrinol Metab 1991;72:51-59.

222. Clemmons DR, Snyder DK, Williams R, Underwood LE. Growth hormone administration conserves lean body mass during dietary restriction in obese subjects. J Clin Endocrinol Metab 1987;64:878-883.

223. Nam SY, Kim KR, Cha BS, Song YD, Lim SK, Lee HC, Huh KB. Low-dose growth hormone treatment combined with diet restriction decreases insulin resistance by reducing visceral fat and increasing muscle mass in obese type 2 diabetic patients. Int J Obes 2001;25:1101-1107.

224. Snyder DK, Clemmons DR, Underwood LE. Dietary carbohydrate content determines responsiveness to growth hormone in energy-restricted humans. J Clin Endocrinol Metab 1989;69:745-752.

225. Gerard JM, Garibaldi L, Myers SE, Aceto Jr T, Kotagal S, Gibbons V, Weber C. Sleep apnea in patients receiving growth hormone. Clin Pediatr 1997;36:321-326.

226. Colditz GA, Willett WC, Rotnitzky A, Manson JE. Weight gain as a risk factor for clinical diabetes mellitus in women. Ann Intern Med 1995;122:481-486.

227. Knowler WC, Barrett-Connor E, Fowler SE, Hamman RF, Lachin JM, Walker EA, Nathan DM. Reduction in the incidence of type 2 diabetes with lifestyle intervention or metformin. $\mathrm{N}$ Engl $\mathrm{J}$ Med 2002;346:393-403.

228. Poirier P, Despres JP. Exercise in weight management of obesity. Cardiol Clin 2001;19:459-470.

229. Wylie-Rosett J, Herman WH, Goldberg RB. Lifestyle intervention to prevent diabetes: intensive AND cost effective. Curr Opin Lipidol 2006;17:37-44.

230. Brownell KD: The LEARN program for weight management 2000. Dallas, Texas, American Health Publishing Company, 2002,

231. Ramachandran A, Snehalatha C, Mary S, Mukesh B, Bhaskar AD, Vijay V, Indian Diabetes Prevention Programme (IDPP). The Indian Diabets Prevention Programme shows that lifestyle modification and metformin prevent type 2 diabets in Asian Indian subjects with impaired glucose tolerance (IDPP-1). Diabetologia 2006;49:289-297.

232. Anonymous. Effect of intensive blood-glucose control with metformin on complications in overweight patients with type 2 diabetes (UKPDS 34). UK Prospective Diabetes Study (UKPDS) Group. Lancet 1998;352:854-865.

233. Dobbins RL, Chester MW, Stevenson BE, Daniels MB, Stein DT, McGarry JD. A fatty acid-dependent step is critically important for both glucose- and non-glucose-stimulated insulin secretion. J Clin Invest 1998;101:2370-2376.

234. Pavo I, Jermendy G, Varkonyi TT, Kerenyi Z, Gyimesi A, Shoustov S, Shestakova M, Herz M, Johns D, Schluchter BJ, Festa A, Tan MH. Effect of pioglitazone compared with metformin on glycemic control and indicators of insulin sensitivity in recently diagnosesd patients with type 2 diabetes. J Clin Endocrinol Metab 2003;88:1637-1645. 
235. Boyle PJ, King AB, Olansky L, Marchetti A, Lau H, Magar R, Martin J. Effects of pioglitazone and rosiglitazone on blood lipid levels and glycemic control in patients with type 2 diabetes mellitus: a retrospective review of randomly selected medical records. Clin Ther 2002;24:378-396.

236. Khan MA, St Peter JV, Xue JL. A prospective, randomized comparison of the metabolic effects of pioglitazone or rosiglitazone in patients with type 2 diabetes who were previously treated with troglitazone. Diabetes Care 2002;25:708-711.

237. Dormandy JA, Charbonnel B, Eckland DJ, Erdmann E, Massi-Benedetti M, Moules IK, Skene AM, Tan MH, Lafebvre PJ, Murray GS, Standl E, Wilcox RG, Wilhelmsen L, Betteridge J, Birkeland K, Golay A, Heine RJ, Koranyi L, Laakso M, Mokan M, Norkus A, Pirags V, Podar T, Scheen A, Scherbaum W, Schernthaner G, Schmitz O, Skrha J, Smith U, Taton J, PROactive investigators. Secondary prevention of macrovascular events in patients with type 2 diabetes in the PROactive Study (PROspective pioglitAzone Clinical Trial in macroVascular Events); a randomised controlled trial. Oancet 2005;366:1279-1289.

238. Buchanan TA, Xiang AH, Peters RK, Kjos SL, Marroquin A, Goico J, Ochoa C, Tan S, Berkowitz K, Hodis HN, Azen SP. Preservation of pancreatic beta-cell function and prevention of type 2 diabetes by pharmacological treatment of insulin resistance in high-risk hispanic women. Diabetes 2002;51:27962803.

239. Xiang AH, Peters RK, Kjos SL, Marroquin A, Goico J, Ochoa C, Kawakubo M, Buchanan TA. Effect of pioglitazone on pancreatic beta-cell function and diabetes risk in hispanic women with prior gestational diabetes. Diabetes 2006;55:517-522.

240. Adams M, Montague CT, Prins JB, Holder JC, Smith SA, Sanders L, Digby JE, Sewter CP, Lazar MA, Chatterjee KK, O’Rahilly S. Activators of peroxisome proliferator-activated receptor $\square$ have depotspecific effects on human preadipocyte differentiation. J Clin Invest 1997;100:3149-3153.

241. Tchoukalova YD, Hausman DB, Dean RG, Hausman GJ. Enhancing effect of troglitazone on porcine adipocyte differentiation in primary culture: a comparison with dexamethasone. Obes Res 2000;8:664672.

242. Laplante M, Sell H, MacNaul KL, Richard D, Berger JP, Deshaies Y. PPAR-gamma activation mediates adipose depot-specific effects on gene expression and lipoprotein lipase activity: mechanisms for modulation of postprandial lipemia and differential adipose accretion. Diabetes 2003;52:291-299.

243. Kageyama H, Hirano T, Okada K, Ebara T, Kageyama A, Murakami T, Shioda S, Adachi M. Lipoprotein lipase mRNA in white adipose tissue but not in skeletal muscle is increased by pioglitazone through PPAR-gamma. Biochem Biophys Res Commun 2003;305:22-27.

244. Chao L, Marcus-Samuels B, Mason MM, Moitra J, Vinson C, Arioglu E, Gavrilova O, Reitman ML. Adipose tissue is required for the antidiabetic, but not for the hypolipidemic, effect of thiazolidinediones. J Clin Invest 2000;106:1221-1228.

245. O’Moore-Sullivan TM, Prins JB. Thiazolidinediones and type 2 diabetes: new drugs for an old disease. Med J Aust 2002;176:381-386.

246. Ye JM, Doyle PJ, Iglesias MA, Watson DG, Cooney GJ, Kraegen EW. Peroxisome proliferator-activated receptor (PPAR)-alpha activaton lowers muscle lipids and improves insulin sensitivity in high fat-fed rats: comparison with PPAR-gamma activation. Diabetes 2001;50:411-417.

247. Sidhu JS, Kaposzta Z, Markus HS, Kaski JC. Effect of rosiglitazone on common carotid intima-media thickness progression in coronary artery disease patients without diabetes mellitus. Arterioscler Thromb Vasc Biol 2004;24:930-934.

248. Al-Salman J, Arjomand H, Kemp DG, Mittal M. Hepatocellular injury in a patient receiving rosiglitazone. A case report. Ann Intern Med 2000;132:121-124.

249. Forman LM, Simmons DA, Diamond RH. Hepatic failure in a patient taking rosiglitazone. Ann Intern Med 2000;132:118-121.

250. May LD, Lefkowitch JH, Kram MT, Rubin DE. Mixed hepatocellular-cholestatic liver injury pioglitazone therapy. Ann Intern Med 2002; 136:449-452.

251. Pinto AG, Cummings OW, Chalasani N. Severe but reversible cholestatic liver injury after pioglitazone therapy. Ann Intern Med 2002;137:857.

252. Neuschwander-Tetri BA. Evolving pathophysiologic concepts in nonalcoholic steatohepatitis. Curr Gastroenterol Rep 2002;4:31-36.

253. Harrison SA, Kadakia S, Lang KA, Schenker S. Nonalcoholic steatohepatitis: what we know in the new millennium. Am J Gastroenterol 2002;97:2714-2724.

254. Jaeschke H, Gores GJ, Cederbaum AI, Hinson JA, Pessayre D, Lemasters JJ. Mechanisms of hepatotoxicity. Toxicol Sci 2002;65:166-176.

255. Yu AS, Keeffe EB. Nonalcoholic fatty liver disease. Rev Gastroenterol Disord 2002;2:11-19. 
256. Luyckx FH, Desaive C, thiry A, Dewe W, Scheen AJ, Gielen JE, Lefebvre PJ. Liver abnormalities in severely obese subjects: effect of drastic weight loss after gastroplasty. Int J Obes Relat Metab Disord 1998;22:222-226.

257. Luyckx FH, Lefebvre PJ, Scheen AJ. Non-alcoholic steatohepatitis: association with obesity and insulin resistance, and influence of weight loss. Diabetes Metab 2000;26:98-106.

258. Caldwell SH, Hespenheide EE, Redick JA, Iezzoni JC, Battle EH, Sheppard BL. A pilot study of a thiazolidinedione, troglitazone, in nonalcoholic steatohepatitis. Am J Gastroenterol 2001;96:519-525.

259. Mayerson AB, Hundal RS, Dufour S, Lebon V, Befroy D, Cline GW, Enocksson S, Inzucchi SE, Shulman GI, Petersen KF. The effects of rosiglitazone on insulin sensitivity, lipolysis, and hepatic and skeletal muscle triglyceride contents in patients with type 2 diabetes. Diabetes 2002;51:797-802.

260. Kelly IE, Han TS, Walsh K, Lean MEJ. Effects of a thiazolidinedione compound on body fat and fat distribution of patients with type 2 diabetes. Diabetes Care 1999;22:288-293.

261. Katoh S, Hata S, Matsushima M, Ikemoto S, Inoue Y, Yokoyama J, Tajima N. Troglitazone prevents the rise in visceral adiposity and improves fatty liver associated with sulfonylurea therapy - a randomized controlled trial. Metabolism 1 A.D.;50:414-417.

262. Bajaj M, Suraamornkul S, Pratipanawatr T, Hardies LJ, Pratipanawatr W, Glass L, Cersosimo E, Miyazaki Y, DeFronzo RA. Pioglitazone reduces hepatic fat content and augments splanchnic glucose uptake in patients with type 2 diabetes. Diabetes 2003;52:1364-1370.

263. Boelsterli UA, Bedoucha M. Toxicological consequences of altered peroxisome proliferator-activated receptor $\gamma(\operatorname{PPAR} \gamma)$ expression in the liver: insights from models of obesity and type 2 diabetes. Biochem Pharmacol 2002;63:1-10.

264. Bedoucha M, Atzpodien E, Boelsterli UA. Diabetic KKAy mice exhibit increased hepatic PPARgammal gene expression and develop hepatic steatosis upon chronic treatment with antidiabetic thiazolidinediones. J Hepatol 2001;35:17-23.

265. Moore GB, Chapman H, Holder JC, Lister CA, Piercy V, Smith SA, Clapham JC. Differential regulation of adipocytokine mRNAs by rosiglitazone in db/db mice. Biochem Biophys Res Commun 2001;286:735741.

266. Hartman HB, Hu X, Tyler KX, Dalal CK, Lazar MA. Mechanisms regulating adipocyte expression of resistin. J Biol Chem 2002;277:19754-19761.

267. Yu JG, Javorschi S, Hevener AL, Kruszynska YT, Norman RA, Sinha M, Olefsky JM. The effect of thiazolidinediones on plasma adiponectin levels in normal, obese, and type 2 diabetic subjects. Diabetes 2002;51:2968-2974.

268. Willi SM, Kennedy A, Wallace P, Ganaway E, Rogers NL, Garvey WT. Troglitazone antagonizes metabolic effects of glucocorticoids in humans: effects on glucose tolerarnce, insulin sensitivity, sduppression of free fatty acids, and leptin. Diabetes 2002;51:2895-2902.

269. Shadid S, Jensen MD. Angioneurotic edema as a side effect of pioglitazone. Diabetes Care 2002;25:405.

270. Cobelli C, Caumo A, Omenetto $M$. Minimal model $S_{G}$ overestimation and $S_{I}$ underestimation: improved accuracy by a Bayesian two-compartment model. Am J Physiol 1999;277:E481-E488.

271. Bergman RN, Ider YZ, Bowden CR, Cobelli C. Quantitative estimation of insulin sensitivity. Am J Physiol 1979;236:E667-E677.

272. Mittendorfer B, Liem O, Patterson BW, Miles JM, Klein S. What does the measurement of whole-body fatty acid rate of appearance in plasma by using a fatty acid tracer really mean? Diabetes 2003;52:16411648.

273. Coon PJ, Rogus EM, Drinkwater DT, Muller DC, Goldberg AP. Role of body fat distribution in the decline in insulin sensitivity and glucose tolerance with age. J Clin Endocrinol Metab 1992;75:1125-1132.

274. Peiris AN, Struve MF, Mueller RA, Lee MB, Kissebah AH. Glucose metabolism in obesity: influence of body fat distribution. J Clin Endocrinol Metab 1988;67:760-767.

275. Havel RJ, Carlson LA, Ekelund L, Holmgren A. Turnover rate and oxidation of different free fatty acids in man during exercise. J Appl Physiol 1964;19:613-618.

276. Boden G, Chen X, Ruiz J, White JV, Rossetti L. Mechanisms of fatty acid-induced inhibition of glucose uptake. J Clin Invest 1994;93:2438-2446.

277. Rebrin K, Steil GM, Mittelman SD, Bergman RN. Casual linkage between insulin suppression of lipolysis and suppression of liver glucose output in dogs. J Clin Invest 1996;98:741-749.

278. Lewis GF, Uffelman KD, Szeto LW, Weller B, Steiner G. Interaction between free fatty acids and insulin in the acute control of very low density lipoprotein production in humans. J Clin Invest 1995;95:158-166. 
279. Steinberg HO, Tarshoby M, Monestel R, Hook G, Cronin J, Johnson A, Bayazeed B, Baron AD. Elevated circulating free fatty acid levels impair endothelium-dependent vasodilation. $\mathrm{J}$ Clin Invest 1997; 100:1230-1239.

280. Kanaley JA, Andresen-Reid ML, Oenning L, Kottke BA, Jensen MD. Differential health benefits of weight loss in upper-body and lower-body obese women. Am J Clin Nutr 1993;57:20-26.

281. Miyazaki Y, Mahankali A, Matsuda M, Mahankali S, Hardies J, Cusi K, Mandarino LJ, DeFronzo RA. Effect of pioglitazone on abdominal fat distribution and insulin sensitivity in type 2 diabetic patients. $\mathrm{J}$ Clin Endocrinol Metab 2002;87:2784-2791.

282. Miles JM, Wooldridge D, Grellner WJ, Windsor S, Isley WL, Klein S, Harris WS. Nocturnal and postprandial free fatty acid kinetics in normal and type 2 diabetic subjects: effects of insulin sensitization therapy. Diabetes 2003;52:675-681.

283. Boden G, Cheung P, Mozzoli M, Fried SK. Effect of thiazolidinediones on glucose and fatty acid metabolism in patients with type 2 diabetes. Metabolism 2003;52:753-759.

284. Racette SB, Davis AO, McGill JB, Klein S. Thiazolidinediones enhance insulin-mediated suppression of fatty acid flux in type 2 diabetes mellitus. Metabolism 2002;51:169-174.

285. Firth RG, Bell PM, Rizza RA. Effects of tolazamide and exogenous insulin on insulin action in patients with non-insulin-dependent diabetes mellitus. N Engl J Med 1986;314:1280-1286.

286. Botion LM, Green A. Long-term regulation of lipolysis and hormone-sensitive lipase by insulin and glucose. Diabetes 1999;48:1691-1697.

287. Smith U. Studies of human adipose tissue in culture III: influence of insulin and medium glucose concentration on cellular metabolism. J Clin Invest 1974;53:91-98.

288. Shadid S, Jensen MD. Effects of pioglitazone vs diet and exercise on metabolic health and fat distribution in upper body obesity. Diabetes Care 2003;26:3148-3152.

289. Folch J, Lees M, Sloane Stanley GH. A simple method for the isolation and purification of total lipids from animal tissues. J Biol Chem 1957;226:497-509.

290. Hevener AL, Reichart D, Janez A, Olefsky J. Thiazolidinedione treatment prevents free fatty acidinduced insulin resistance in male Wistar rats. Diabetes 2001;50:2316-2322.

291. Jensen MD, Chandramouli V, Schumann WE, Ekberg K, Previs SF, Gupta S, Landau BR. Sources of blood glycerol during fasting. Am J Physiol Endocrinol Metab 2001;281:E998-E1004.

292. Jensen MD, Caruso M, Heiling V, Miles JM. Insulin regulation of lipolysis in nondiabetic and IDDM subjects. Diabetes 1989;38:1595-1601.

293. Jensen MD. Regulation of forearm lipolysis in different types of obesity. In vivo evidence for adipocyte heterogeneity. J Clin Invest 1991;87:187-193.

294. Rebrin K, Steil GM, Getty L, Bergman RN. Free fatty acids as a link in the regulation of hepatic glucose output by peripheral insulin. Diabetes 1995;44:1038.

295. Coppack SW, Evans RD, Fisher RM, Frayn KN, Gibbons GF, Humphreys SM, Kirk ML, Potts JL, Hockaday TDR. Adipose tissue metabolism in obesity: lipase action in vivo before and after a mixed meal. Metabolism 1992;41:264-272.

296. Havel RJ, Kane JP, Balasse EO, Segel N, Basso LV. Splanchnic metabolism of free fatty acids and production of triglycerides of very low density lipoproteins in normotriglyceridemic and hypertriglyceridemic humans. J Clin Invest 1970;49:2017-2035.

297. Nagashima K, Lopez C, Donovan D, Ngai C, Fontanez N, Bensadoun A, Fruchart-Najib J, Holleran S, Cohn JS, Ramakrishnan R, Ginsberg HN. Effects of the PPAR $\square$ agonist pioglitazone on lipoprotein metabolism in patients with type 2 diabetes mellitus. J Clin Invest 2005;115:1323-1332.

298. Coppack SW, Persson M, Judd RL, Miles JM. Glycerol and nonesterified fatty acid metabolism in human muscle and adipose tissue in vivo. Am J Physiol 1999;276:E233-E240.

299. Dea MK, Van Citters GW, Ader M, Mittelman SD, Sunehag AL, Bergman RN. Paradoxical effect of troglitazone in normal animals: enhancement of adipocyte by reduction of liver insulin sensitivity. Diabetes 2000;49:2087-2093.

300. Tiikkainen M, Häkkinen A-M, Korsheninnikova E, Nyman T, Mäkimattila S, Yki-Jarvinen H. Effects of rosiglitazone and metformin on liver fat content, hepatic insulin resistance, insulin clearance, and gene expression in adipose tissue in patients with type 2 diabetes. Diabetes 2004;53:2169-2176.

301. Coppack SW. Pro-inflammatory cytokines and adipose tissue. Proc Nutr Soc 2001;60:349-356.

302. Pepys MB, Hirschfield GM. C-reactive protein: a critical update. J Clin Invest 2003;111:1805-1812.

303. Shadid S, Jensen MD. Effects of pioglitazone vs diet and exercise on metabolic health and fat distribution in upper body obesity. Diabetes Care 2003;26:3148-3152. 
304. Marfella R, Esposito K, Siniscalchi M, Cacciapuoti F, Giugliano F, Labriola D, Ciotola M, Di Palo C, Misso L, Giugliano D. Effect of weight loss on cardiac synchronization and proinflammatory cytokines in premenopausal obese women. Diabetes Care 2004;27:47-52.

305. Haffner SM, Greenberg AS, Weston WM, Chen H, Williams K, Freed MI. Effect of rosiglitazone treatment on nontraditional markers of cardiovascular disease in patients with type 2 diabetes mellitus. Circulation 2002;106:679-684.

306. Koistinen HA, Bastard JP, Dusserre E, Ebeling P, Zegari N, Andreelli F, Jardel C, Donner M, Meyer L, Moulin P, Hainque B, Riou JP, Laville M, Koivisto VA, Vidal H. Subcutaneous adipose tissue expression of tumour necrosis factor-alpha is not associated with whole body insulin resistance in obese nondiabetic of in type-2 diabetic subjects. Eur J Clin Invest 2000;30:302-310.

307. Saijo Y, Kiyota N, Kawasaki Y, Miyazaki T, Kashimura J, Fukuda M, Kishi R. Relationship between Creactive protein and visceral adipose tissue in healthy Japanese subjects. Diabetes Obes Metab 2004;6:249-258.

308. Kern PA, Ranganathan S, Li C, Wood L, Ranganathan G. Adipose tissue tumor necrosis factor and interleukin-6 expression in human obesity and insulin resistance. Am J Physiol Endocrinol Metab 2001;280:E745-E751.

309. Carey AL, Febbraio MA. Interleukin-6 and insulin sensitivity: friend or foe? Diabetologia 2004;47:11351142 .

310. Mohamed-Ali V, Goodrick S, Rawesh A, Katz DR, Miles JM, Yudkin JS, Klein S, Coppack SW. Subcutaneous adipose tissue releases interleukin-6, but not tumor necrosis factor-alpha, in vivo. J Clin Endocrinol Metab 1997;82:4196-4200.

311. Winkler G, Kiss S, Keszthelyi L, Sapi Z, Ory I, Salamon F, Kovacs M, Vargha P, Szekeres O, Speer G, Karadi I, Sikter M, Kaszas E, Dworak O, Gero G, Cseh K. Expression of tumor necrosis factor (TNF)alpha protein in the subcutaneous and visceral adipose tissue in correlation with adipocyte cell volume, serum TNF-alpha, soluble serum TNF-receptor-2 concentrations and C-peptide level. Eur J Endocrinol 2003;149:129-135.

312. Monzillo LU, Hamdy O, Horton ES, Ledbury S, Mullooly C, Jarema C, Porter S, Ovalle K, Moussa A, Mantzoros CS. Effect of lifestyle modification on adipokine levels in obese subjects with insulin resistance. Obes Res 2003;11:1048-1054.

313. Azuma K, Katsukawa F, Oguchi S, Murata M, Yamazaki H, Shimada A, Saruta T. Correlation between serum resistin level and adiposity in obese individuals. Obes Res 2003;11:997-1001.

314. Silha JV, Krsek M, Skrha JV, Sucharda P, Nyomba BL, Murphy LJ. Plasma resistin, adiponectin and leptin levels in lean and obese subjects: correlations with insulin resistance. Eur $\mathrm{J}$ Endocrinol 2003;149:331-335.

315. Stejskal D, Adamovska S, Bartek J, Jurakova R, Proskova J. Resistin - concentrations in persons with type 2 diabetes mellitus and in individuals with acute inflammatory disease. Biomed Pap Med Fac Univ Palacky Olomouc Czech Repub 2003;147:63-69.

316. Valsamakis G, McTernan PG, Chetty R, Al Daghri N, Field A, Hanif W, Barnett AH, Kumar S. Modest weight loss and reduction in waist circumference after medical treatment are associated with favorable changes in serum adipocytokines. Metabolism 2004;53:430-434.

317. Heilbronn LK, Rood J, Janderova L, Albu JB, Kelley DE, Ravussin E, Smith SR. Relationship between serum resistin concentrations and insulin resistance in nonobese, obese, and obese diabetic subjects. $\mathrm{J}$ Clin Endocrinol Metab 2004;89:1844-1848.

318. Ort T, Arjona AA, MacDougall JR, Nelson PJ, Rothenberg ME, Wu F, Eisen A, Halvorsen YD. Recombinant human FIZZ3/resistin stimulates lipolysis in cultured human adipocytes, mouse adipose explants, and normal mice. Endocrinology 2005;146:2200-2209.

319. Yang WS, Jeng CY, Wu TJ, Tanaka S, Funahashi T, Matsuzawa Y, Wang JP, Chen CL, Tai TY, Chuang LM. Synthetic peroxisome proliferator-activated receptor-gamma agonist, rosiglitazone, increases plasma levels of adiponectin in type 2 diabetic patients. Diabetes Care 2002;25:376-380.

320. Phillips SA, Ciaraldi TP, Kong AP, Bandukwala R, Aroda V, Carter L, Baxi S, Mudaliar SR, Henry RR. Modulation of circulating and adipose tissue adiponectin levels by antidiabetic therapy. Diabetes 2003;52:667-674.

321. Satoh N, Ogawa Y, Usui T, Tagami T, Kono S, Uesugi H, Sugiyama H, Sugawara A, Yamada K, Shimatsu A, Kuzuya H, Nakao K. Antiatherogenic effect of pioglitazone on type 2 diabetic patients irrespective of the responsiveness to its antidiabetic effect. Diabetes Care 2003;26:2493-2499. 
322. Yu JG, Javorschi S, Hevener AL, Kruszynska YT, Norman RA, Sinha M, Olefsky JM. The effect of thiazolidinediones on plasma adiponectin levels in normal, obese, and type 2 diabetic subjects. Diabetes 2002;51:2968-2974.

323. Pajvani UB, Hawkins M, Combs TP, Rajala MW, Doebber T, Berger JP, Wagner JA, Wu M, Knopps A, Xiang AH, Utzschneider KM, Kahn SE, Olefsky JM, Buchanan TA, Scherer PE. Complex distribution, not absolute amount of adiponectin, correlates with thiazolidinedione-mediated improvement in insulin sensitivity. J Biol Chem 2004;279:12152-12162.

324. Fisher FF, Trujillo ME, Hanif W, Barnett AH, McTernan PG, Scherer PE, Kumar S. Serum high molecular weight complex of adiponectin correlates better with glucose tolerance than total serum adiponectin in Indo-Asian males. Diabetologia 2005;48:1084-1087.

325. Abbasi F, Chang SA, Chu JW, Ciaraldi TP, Lamendola C, McLaughlin T, Reaven GM, Reaven PD. Improvements in insulin resistance with weight loss, in contrast to rosiglitazone, are not associated with changes in plasma adiponectin or adiponectin multimeric complexes. Am J Physiol Regul Integr Comp Physiol 2006;290:R139-R144.

326. Schaffler A, Herfarth H, Paul G, Ehling A, Muller-Ladner U, Scholmerich J, Zietz B. Identification of influencing variables on adiponectin serum levels in diabetes mellitus type 1 and type 2. Exp Clin Endocrinol Diabetes 2004;112:383-389.

327. Vendrell J, Broch M, Vilarrasa N, Molina A, Gómez JM, Gutiérrez C, Simón I, Soler J, Richart C. Resistin, adiponectin, ghrelin, leptin, and proinflammatory cytokines: relationships in obesity. Obes Res 2004;12:962-971.

328. Xu A, Chan KW, Hoo RL, Wang Y, Tan KC, Zhang J, Chen B, Lam MC, Tse C, Cooper GJ, Lam KS. Testosterone selectively reduces the high molecular weight form of adiponectin by inhibiting its secretion from adipocytes. J Biol Chem 2005;280:18073-18080.

329. Esposito K, Pontillo A, Di Palo C, Giugliano G, Masella M, Marfella R, Giugliano D. Effect of weight loss and lifestyle changes on vascular inflammatory markers in obese women: a randomized trial. J Am Med Assoc 2003;289:1799-1804.

330. Kopp HP, Kopp CW, Resta A, Krzyzanowska K, Kriwanek S, Minar E, Roka R, Schernthaner G. Impact of weight loss on inflammatory proteins and their association with the insulin resistance syndrome in morbidly obese patients. Arterioscler Thromb Vasc Biol 2003;23:1042-1047.

331. Mohanty P, Aljada A, Ghanim H, Hofmeyer D, Tripathy D, Syed T, Al-Haddad W, Dhindsa S, Dandona P. Evidence for a potent antiinflammatory effect of rosiglitazone. J Clin Endocrinol Metab 2004;89:27282735 .

332. Tonelli J, Li W, Kishore P, Pajvani UB, Kwon E, Weaver C, Scherer PE, Hawkins M. Mechanisms of early insulin-sensitizing effects of thiazolidinediones in type 2 diabetes. Diabetes 2004;53:1621-1629.

333. Bergman RN, Ider YZ, Bowden CR, Cobelli C. Quantitative estimation of insulin sensitivity. Am J Physiol 1979;236:E667-E677.

334. Cava F, Gonzalez C, Pascual MJ, Navajo JA, Gonzalez-Buitrago JM. Biological variation of interleukin 6 (IL-6) and soluble interleukin 2 receptor (sIL2R) in serum of healthy individuals. Cytokine 2000;12:1423-1425.

335. Hak AE, Stehouwer CD, Bots ML, Polderman KH, Schalkwijk CG, Westendorp IC, Hofman A, Witteman JC. Associations of C-reactive protein with measures of obesity, insulin resistance, and subclinical atherosclerosis in healthy, middle-aged women. Arterioscler Thromb Vasc Biol 1999;19:1986-1991.

336. Firth RG, Bell PM, Rizza RA. Effects of tolazamide and exogenous insulin on insulin action in patients with non-insulin-dependent diabetes mellitus. N Engl J Med 1986;314:1280-1286.

337. Botion LM, Green A. Long-term regulation of lipolysis and hormone-sensitive lipase by insulin and glucose. Diabetes 1999;48:1691-1697.

338. Smith U. Studies of human adipose tissue in culture III: influence of insulin and medium glucose concentration on cellular metabolism. J Clin Invest 1974;53:91-98.

339. Putz DM, Goldner WS, Bar RS, Haynes WG, Sivitz WI. Adiponectin and C-reactive protein in obesity, type 2 diabetes, and monodrug therapy. Metabolism 2004;53:1454-1461.

340. Lamarche B, Lemieux I, Despres JP. The small, dense LDL phenotype and the risk of coronary heart disease: epidemiology, patho-physiology and therapeutic aspects. Diabetes Metab 1999;25:199-211.

341. Sacks FM, Campos H. Low-Density lipoprotein size and cardiovascular disease: a reappraisal. J Clin Endocrinol Metab 2003;88:4525-4532.

342. Berneis KK, Krauss RM. Metabolic origins and clinical significance of LDL heterogeneity. J Lipid Res 2002;43:1363-1379. 
343. Blackburn P, Lemieux I, Lamarche B, Bergeron J, Perron P, Tremblay G, Gaudet D, Despres JP. Effect of type 2 diabetes on various electrophoretic characteristics of low-density lipoprotein particles in women. Diabetologia 2004; Epub ahead of print:

344. Wagner AM, Jorba O, Rigla M, Bonet R, de Leiva A, Ordonez-Llanos J, Perez A. Effect of improving glycemic control on low-density lipoprotein particle size in type 2 diabetes. Metabolism 2003;52:15761578 .

345. Winkler K, Konrad T, Fullert S, Friedrich I, Destani R, Baumstark MW, Krebs K, Wieland H, Marz W. Pioglitazone reduces atherogenic dense LDL particles in nondiabetic patients with arterial hypertension: a double-blind, placebo-controlled study. Diabetes Care 2003;26:2588-2594.

346. Promrat K, Lutchman G, Kleiner DE, Uwaifo GI, Freedman RJ, Soza A, Heller T, Doo E, Ghany M, Park Y, Yanovski JA, Liang TJ, Hoofnagle JH: Pilot study of pioglitazone in nonalcoholic steatohepatitis (Abstract). Gastroenterology 124: Suppl, 2003

347. Sarafidis PA, Lasaridis AN, Nilsson PM, Pagkalos EM, Hitoglou-Makedou AD, Pliakos CI, Kazakos KA, Yovos JG, Zebekakis PE, Tziolas IM, Tourkantonis AN. Ambulatory blood pressure reduction after rosiglitazone treatment in patients with type 2 diabetes and hypertension correlates with insulin sensitivity increase. J Hypertens 2004;22:1769-1777.

348. Goldberg RB, Kendall DM, Deeg MA, Buse JB, Zagar AJ, Pinaire JA, Tan MH, Khan MA, Perez AT, Jacober SJ, for the GLAI Study Investigators. A comparison of lipid and glycemic effects of pioglitazone and rosiglitazone in patients with type 2 diabtes and dyslipidemia. Diabetes Care 2005;28:1547-1554.

349. Friedewald WT, Levy RI, Fredrickson DS. Estimation of the concentration of low-density lipoprotein cholesterol in plasma, without use of the preparative ultracentrifuge. Clin Chem 1972;18:499-502.

350. Otvos JD. Measurement of lipoprotein subclass profiles by nuclear magnetic resonance spectroscopy. Clin Lab 2002;48:171-180.

351. Festa, A., Williams, K., Hanley, A. J. G., Otvos, J. D., Goff, D. C., Wagenknecht, L. E., and Haffner, S. M. Nuclear magnetic resonance (NMR) lipoprotein abnormalities in prediabetic subjects in the insulin resistance atherosclerosis study (IRAS). Circulation . 2005. Ref Type: In Press

352. Harder H, Dinesen B, Astrup A. The effect of a rapid weight loss on lipid profile and glycemic control in obese type 2 diabetic patients. Int J Obes 2004;28:180-182.

353. Kraus WE, Houmard JA, Duscha BD, Knetzger KJ, Wharton MB, McCartney JS, Bales CW, Henes S, Samsa GP, Otvos JD, Kulkarni KR, Slentz CA. Effects of the amount and intensity of exercise on plasma lipoproteins. N Engl J Med 2002;347:1492.

354. Carr MC, Hokanson JE, Zambon A, Deeb SS, Barrett PH, Purnell JQ, Brunzell JD. The contribution of intraabdominal fat to gender differences in hepatic lipase activity and low/high density lipoprotein heterogeneity. J Clin Endocrinol Metab 2001;86:2831-2837.

355. Bogacka I, Xie H, Bray GA, Smith SR. The effect of pioglitzaone on peroxisome proliferator-activated receptor-gamma target genes related to lipid storage in vivo. Diabetes Care 2004;27:1660-1667.

356. Lewis GF, Murdoch S, Uffelman K, Naples M, Szeto L, Albers A, Adeli K, Brunzell JD. Hepatic lipase mRNA, protein, and plasma enzyme activity is increased in the insulin-resistant, fructose-fed Syrian golden hamster and is partially normalized by the insulin sensitizer rosiglitazone. Diabetes 2004;53:28932900 .

357. Wilmsen HM, Ciaraldi TP, Carter L, Reehman N, Mudaliar SR, Henry RR. Thiazolidinediones upregulate impaired fatty acid uptake in skeletal muscle of type 2 diabetic subjects. Am J Physiol Endocrinol Metab 2003;285:E354-E362.

358. Cromwell WC, Otvos JD. Low-density lipoprotein particle number and risk for cardiovascular disease. Curr Atheroscler Rep 2004;6:381-387.

359. Ross R, Rissanen J, Pedwell H, Clifford J, Shragge P. Influence of diet and exercise on skeletal muscle and visceral adipose tissue in men. J Appl Physiol 1996;81:2445-2455.

360. Kawai T, Takei I, Oguma Y, Ohashi N, Tokui M, Oguchi S, Katsukawa F, Hirose H, Shimada A, Watanabe K, Saruta T. Effects of troglitazone on fat distribution in the treatment of male type 2 diabetes. Metabolism 1999;48:1102-1107.

361. Akazawa S, Sun F, Ito M, Kawasaki E, Eguchi K. Efficacy of troglitazone on body fat distribution in type 2 diabetes. Diabetes Care 2000;23:1067-1071.

362. Mori Y, Murakawa Y, Okada K, Horikoshi H, Yokoyama J, Tajima N, Ikeda Y. Effect of troglitazone on body fat distribution in Type 2 diabetic patients. Diabetes Care 1999;22:908-912.

363. de Souza CJ, Eckhardt M, Gagen K, Dong M, Chen W, Laurent D, Burkey BF. Effects of pioglitazone on adipose tissue remodeling within the setting of obesity and insulin resistance. Diabetes 2001;50:18631871 . 
364. Di Girolamo M, Fine JB. Cellularity measurements. Methods Mol Biol 2001;155:65-75.

365. Wang J, Pierson RN. Disparate hydration of adipose and lean tissue require a new model for body water distribution in man. J Nutr 1976;106:1687-1693.

366. Smith SR, Zachwieja JJ. Visceral adipose tissue: a critical review of intervention strategies. Int J Obes 1999;23:329-335.

367. Bonen A, Luiken JJ, Glatz JF. Regulation of fatty acid transport and membrane transporter in health and disease. Mol Cell Biochem 2002;239:181-192.

368. Stahl A, Evans JG, Pattel S, Hirsch D, Lodish HF. Insulin causes fatty acid transport protein translocation and enhanced fatty acid uptake in adipocytes. Dev Cell 2002;2:477-488.

369. van Wijk JP, de Koning EJ, Castro Cabezas M, Rabelink TJ. Rosiglitazone improves postprandial triglyceride and free fatty acid metabolism in type 2 diabetes. Diabetes Care 2005;28:844-849.

370. Gegick CG, Altheimer MD. Thiazolidinediones: comparison of long-term effects on glycemic control and cardiovascular risk factors. Curr Med Res Opin 2004;20:919-930.

371. Robinson AC, Jeffs JA, Gray RG, Bannister PA, Mather H, Gallagher JJ, Robinson S, Nattrass M, Venkatesan S, Halliday D, Johnston DG. Metabolic effects of troglitazone in patient with diet-controlled type 2 diabetes. Eur J Clin Invest 2004;34:29-36.

372. Boden G, Homko C, Mozzoli M, Showe LC, Nichols C, Cheung P. Thiazolidinediones upregulate fatty acid uptake and oxidation in adipose tissue of diabetic patients. Diabetes 2005;54:880-885.

373. Coort SL, Coumans WA, Bonen A, van der Vusse GJ, Glatz JF, Luiken JJ. Divergent effects of rosiglitazone on protein-mediated fatty acid uptake in adipose and in muscle tissues of Zucker rats. J Lipid Res 2005;46:1295-1302.

374. Fisher FF, Trujillo ME, Hanif W, Barnett AH, McTernan PG, Scherer PE, Kumar S. Serum high molecular weight complex of adiponectin correlates better with glucose tolerance than total serum adiponectin in Indo-Asian males. Diabetologia 2005;48:1084-1087.

375. Xu A, Chan KW, Hoo RL, Wang Y, Tan KC, Zhang J, Chen B, Lam MC, Tse C, Cooper GJ, Lam KS. Testosterone selectively reduces the high molecular weight form of adiponectin by inhibiting its secretion from adipocytes. J Biol Chem 2005;280:18073-18080.

376. Seftel AD. Testosterone selectively reduces the high molecular weight form of adiponectin by inhibiting its secretion from adipocytes. J Urol 2005;174:1045-1046.

377. Pajvani UB, Hawkins M, Combs TP, Rajala MW, Doebber T, Berger JP, Wagner JA, Wu M, Knopps A, Xiang AH, Utzschneider KM, Kahn SE, Olefsky JM, Buchanan TA, Scherer PE. Complex distribution, not absolute amount of adiponectin, correlates with thiazolidinedione-mediated improvement in insulin sensitivity. J Biol Chem 2004;279:12152-12162.

378. Lautamäki R, Rönnemaa T, Airaksinen KEJ, Leino A, Hiekkanen H, Stewart M, Knuuti J, Nuutila P: Rosiglitazone increases the concentration of large, buoyant but not small dense LDL in patients with type 2 diabetes and coronary artery disease, a 16 week randomised, double-blind, placebo-controlled study (Abstract). Diabetes 54: Suppl 2, 2005

379. Derosa G, D'Angelo A, Ragonesi PD, Ciccarelli L, Piccinni MN, Pricolo F, Salvadeo SA, Montagua L, Gravina A, Ferrari I, Paniga S, Cicero AF. Metformin-pioglitazone and metformin-rosiglitazone effects on non-convential cardiovascular risk factors plasma level in type 2 diabetic patients with metabolic syndrome. J Clin Pharm Ther 2006;31:375-383.

380. Derosa G, Cicero AF, Dangelo A, Gaddi A, Ragonesi PD, Piccinni MN, Salvadeo S, Ciccarelli L, Pricolo F, Ghelfi M, Ferrari I, Montagna L, Fogari R. Thiazolidinedione effects on blood pressure in diabetic patients with metabolic syndrome treated with glimepiride. Hypertens Res 2005;28:917-924.

381. Bajaj M, Suraamornkul S, Hardies LJ, Pratipanawatr T, DeFronzo RA. Plasma resistin concentration, hepatic fat content, and hepatic and peripheral insulin resistance in pioglitzaone-treated type II diabetic patients. Int J Obes Relat Metab Disord 2004;28:783-789.

382. Belfort R, Harrison SA, Brown K, Darland C, Finch J, Hardies J, Balas B, Gastaldelli A, Tio F, Pulcini J, Berria R, Ma JZ, Dwivedi S, Havranek R, Fincke C, DeFronzo R, Bannayan GA, Schenker S, Cusi K. A placebo-controlled trial of pioglitazone in subjects with nonalcoholic steatohepatitis. N Engl J Med 2006;22:2297-2307.

383. Promrat K, Lutchman G, Uwaifo GI, Freedman RJ, Soza A, Heller T, Doo E, Ghany M, Premkumar A, Park Y, Liang TJ, Yanovski JA, Kleiner DE, Hoffnagle JH. A pilot study of pioglitazone treatment for nonalcoholic steatohepatitis. Hepatology 2004;39:188-196.

384. Sanyal AJ, Mofrad PS, Contos MJ, Sargeant C, Luketic VA, terling RK, Stravitz RT, Shiffman ML, Clore J, Mills AS. A pilot study of vitamin E versus vitamin E and pioglitazone for the treatment of nonalcoholic steatohepatitis. Clin Gastroenterol Hepatol 2004;2:1107-1115. 
385. Davidson MB. Is treatment of insulin resistance beneficial independent of glycemia? Diabetes Care 2003;26:3184-3186.

386. Bugianesi E, Zannoni C, Vanni E, Marzocchi R, Marchesini G. Non-alcoholic fatty liver and insulin resistance: a cause-effect relationship? Dig Liver Dis 2004;36:165-173.

387. Seidell JC, Perusse L, Despres JP, Bouchard C. Waist and hip circumferences have independent and opposite effects on cardiovascular disease risk factors: the Quebec Family Study. Am J Clin Nutr 2001;74:315-321.

388. Snijder MB, Dekker JM, Visser M, Bouter LM, Stehouwer CD, Yudkin JS, Heine RJ, Nijpels G, Seidell JC, Hoorn Study. Trunk fat and leg fat have independent and opposite associations with fasting and postload glucose levels: the Hoorn study. Diabetes Care 2004;27:372-377.

389. Van Pelt RE, Jankowski CM, Gozansky WS, Schwartz RS, Kohrt WM. Lower-body adiposity and metabolic protection in postmenopausal women. J Clin Endocrinol Metab 2005;90:4573-4578.

390. He W, Barak Y, Hevener A, Olson P, Liao D, Le J, Nelson M, Ong E, Olefsky JM, Evans RM. Adiposespecific peroxisome proliferator-activated receptor gamma knockout causes insulin resistance in fat and liver but not in muscle. Proc Natl Acad Sci USA 2003;100:15712-15717.

391. Basu A, Jensen MD, McCann F, Mukhopadhyay D, Joyner MJ, Rizza RA. Effects of pioglitazone versus glipizide on body fat distribution, body water content, and hemodynamics in type 2 diabetes. Diabetes Care 2006;29:510-514.

392. Carey DG, Cowin GJ, Galloway GJ, Jones NP, Richards JC, Biswas N, Doddrell DM. Effect of rosiglitazone on insulin sensitivity and body composition in type 2 diabetic patients. Obes Res 2002;10:10081015.

393. Hirose H, Kawai T, Yamamoto Y, Taniyama M, Tomita M, Matsubara K, Okazaki Y, Ishii T, Oguma Y, Takei I, Saruta T. Effects of pioglitazone on metabolic parameters, body fat distribution, and serum adiponectin levels in Japanese male patients with type 2 diabetes. Metabolism 2002;51:314-317.

394. Smith SR, De Jonge L, Volaufova J, Li Y, Xie H, Gray GA. Effect of pioglitazone on body composition and energy expenditure: a randomized controlled trial. Metabolism 2005;54:24-32.

395. Virtanen KA, Hallsten K, Parkkola R, Janatuinen T, Lonnqvist F, Viljanen T, Ronnemaa T, Knuuti J, Huupponen R, Lonnroth P, Nuutila P. Differential effects of rosiglitazone and metformin on adipose tissue distribution and glucose uptake in type 2 diabetic subjects. Diabetes 2003;52:283-290.

396. Abbasi F, Chang SA, Chu JW, Ciaraldi TP, Lamendola C, McLaughlin T, Reaven GM, Reaven PD. Improvements in insulin resistance with weight loss, in contrast to rosiglitazone, are not associated with changes in plasma adiponectin or adiponectin multimeric complexes. Am J Physiol Regul Integr Comp Physiol 2006;290:R139-R144.

397. Panigrahy D, Huang S, Kieran MW, Kaipainen A. PPAR $\square$ as a therapeutic target for tumor angiogenesis and metastasis. Cancer Biol Ther 2005;4:687-693.

398. Palakurthi SS, Aktas H, Grubissich LM, Mortensen RM, Halperin JA. Anticancer effects of thiazolidinediones are independenet of peroxisome proliferator-activated receptor gamma and mediated by inhibition of translation initiation. Cancer Res 2001;61:6213-6218.

399. Karter AJ, Ahmed AT, Liu J, Moffet HH, Parker MM. Pioglitazone initiation and subsequent hospitalization for congestive heart failure. Diabet Med 2005;22:986-993.

400. Rajagopalan R, Rosenson RS, Fernandes AW, Khan M, Murray FT. Association between congestive heart failure and hospitalization in patients with type 2 diabetes mellitus receiving treatment with insulin or pioglitazone: a retrospective data analysis. Clin Ther 2004;26:1400-1410.

401. Delea TE, Edelsberg JS, Hagiwara M, Oster G, Phillips LS. Use of thiazolidinediones and risk of heart failure in people with type 2 diabetes: a retrospective cohort study. Diabetes Care 2003;26:2983-2989.

402. Masoudi FA, Inzucchi SE, Wang Y, Havranek EP, Foody JM, Krumholz HM, . Thiazolidinediones, metformin, and outcomes in older patients with diabetes and heart failure: an observational study. Circulation 2005;111:583-590.

403. Martens FM, Visseren FL, de Koning EJ, Rabelink TJ. Short-term pioglitazone treatment improves vascular function irrespective of metabolic changes in patients with type 2 diabetes. J Cardiovasc Pharmacol 2005;46:773-778.

404. Horio T, Suzuki M, Suzuki K, Takamisawa I, Hiuge A, Kamide K, Takiuchi S, Iwashima Y, Kihara S, Funahashi T, Yoshimasa Y, Kawano Y. Pioglitazone improves left ventricular diastolic function in patients with essential hypertension. Am J Hypertens 2005;18:949-957.

405. Huang A, Raskin P. Thiazolidinediones and insulin: rationale for use and role of combination therapy in type 2 diabetes mellitus. Treat Endocrinol 2005;4:205-220. 
406. Maru S, Koch GG, Stender M, Clark D, Gibowski L, Petri H, White AD, Simpson RJ, Jr. Antidiabetic drugs and heart failure risk in patients with type 2 diabetes in the U.K. primary care setting. Diabetes Care 2005;28:20-26.

407. Nesto RW, Bell D, Bonow RO, Fonseca V, Grundy SM, Horton ES, Le Winter M, Porte D, Semenkovich CF, Smith S, Young LH, Kahn R. Thiazolidinedione use, fluid retention, and congestive heart failure: a consensus statement from the American Heart Association and American Diabetes Association. Diabaetes Care 2004;27:256-263. 


\section{Appendices}

\section{Abbreviations}

$\begin{array}{ll}\text { ANOVA } & \text { Analysis Of Variance } \\ \text { AT } & \text { Adipose Tissue } \\ \text { BMI } & \text { Body Mass Index } \\ \text { BP } & \text { Blood Pressure } \\ \text { CHD } & \text { Coronary Heart Disease } \\ \text { Chol } & \text { Cholesterol } \\ \text { CPT-1 } & \text { Carnitine-Palmitoyl Transferase 1 } \\ \text { CT } & \text { Computer Tomography } \\ \text { Diet/Ex } & \text { Diet and Exercise } \\ \text { DEXA } & \text { Dual Energy X-Ray Absorptiometry } \\ \text { DM } & \text { Diabetes Mellitus } \\ \text { Dpm } & \text { Disintegrations Per Minute } \\ \text { FA } & \text { Fatty Acid(s) } \\ \text { FFA } & \text { Free Fatty Acid(s) } \\ \text { FFM } & \text { Fat Free Mass } \\ \text { GCRC } & \text { General Clinical Research Center } \\ \text { GIR } & \text { Glucose Infusion Rate } \\ \text { HDL } & \text { High Density Lipoprotein } \\ \text { HDL-C } & \text { HDL cholesterol } \\ \text { HPLC } & \text { High Performance (pressure) Liquid Chromatography } \\ \text { IDL } & \text { Intermediate density lipoprotein } \\ \text { Im } & \text { intra-myocyte } \\ \text { IR } & \text { Insulin Resistance } \\ \text { Is } & \text { Insulin Sensitivity } \\ \text { IVGTT } & \text { Intravenous Glucose Tolerance Test } \\ \text { LBO } & \text { Lower Body Obesity/Overweight } \\ \text { LCAC } & \text { Long-Chain Acyl Carnitine } \\ \text { LDL } & \text { Low Density Lipoprotein } \\ \text { LDL-C } & \text { LDL-cholesterol } \\ \text { LDL-P } & \text { LDL particles } \\ \text { LPL } & \text { Lipoprotein Lipase } \\ \text { NAFLD } & \text { Non-Alcoholic Fatty Liver Disease } \\ \text { NEFA } & \text { Non-Esterified Fatty Acid } \\ \text { PIO } & \text { Pioglitazone } \\ \text { PPAR } & \text { Peroxisome Proliferator-Activated Receptor } \\ \text { Ra } & \text { Rate Of Appearance } \\ \text { Rd } & \text { Rate Of Disappearance } \\ \end{array}$




$\begin{array}{ll}\text { REE } & \text { Resting Energy Expenditure } \\ \text { SA } & \text { Specific Activity } \\ \text { SC } & \text { subcutaneous (ly) } \\ \text { Si } & \text { Sensitivity Index (to insulin) } \\ \text { T2DM } & \text { Type 2 Diabetes } \\ \text { TG } & \text { Triglyceride (s) } \\ \text { TNF } & \text { Tumor Necrosis Factor } \\ \text { TZD } & \text { Thiazolidinedione } \\ \text { UBO } & \text { Upper Body Obese/Obesity } \\ \text { VISC } & \text { Visceral } \\ \text { VLDL } & \text { Very Low Density Lipoprotein } \\ \text { WHR } & \text { Waist-to-Hip Ratio }\end{array}$

\section{Conversion Tables}

\begin{tabular}{lll}
\hline & From $\mathbf{~ m g / d l}$ to $\mathbf{~ m m o l} / \mathbf{l}$ & From $\mathbf{~ m o l} / \mathbf{l}$ to $\mathbf{~ m g} / \mathbf{d l}$ \\
\hline Glucose & divide by 18 or multiply by & multiply by 18 \\
& 0.055 & \\
TG & divide by 89 & multiply by 89 \\
LDL and HDL & divide by 39 & multiply by 39 \\
cholesterol & & \\
\hline
\end{tabular}

\section{Exercise Program}

\section{General Instructions}

You can choose your own preferred physical activity (as long as your heart rate reaches the desired level)

Walking up and down the stairs until the heart rate reaches your target level can be done at work or during lunch breaks

Remember that $3 \times 5=15$

STRETCHING is important even when walking is your exercise of choice. It will help you to avoid unnecessary injuries and to complete the program without difficulties

You may do more than advised but be careful in the beginning

Keep a logbook as instructed; Fax weekly to. . . or e-mail to ... 


\section{Personal Program}

Stop and stretch after 5 min and in the end*

Week 1: $3 \times 15-20 \mathrm{~min}$

Week 2+3: 3 × $30 \mathrm{~min}$

Week $3+4: 3 \times 30 \mathrm{~min}$

Weeks $5,6+7: 3 \times 40 \mathrm{~min}$

Weeks 8-12: 3-4 x 40-45 min

Week 12-16: 4 x $50 \mathrm{~min}$

*) see pictures

$\begin{array}{ll}\text { HR } & (50 \% \text { of heart rate reserve) } \\ \text { HR } & (50 \%) \\ \text { HR } & (60 \%) \\ \text { HR } & (60-70 \%) \\ \text { HR } & (60-70 \%) \\ \text { HR } & (60-70 \%)\end{array}$

\section{Behavior Modification Program}

This program was written by Scott Crow, MD, a psychiatrist from the University of Minnesota, Minneapolis, and modified by Samyah Shadid, MD. It consists of 6 plus 2 basic sessions, given at 2-4 week intervals according to the volunteer's needs.

The main aims of the programs are:

- to promote life style changes rather than diets

- to educate the volunteer on the health hazards of overweight and the additional benefits of physical activity

- to teach volunteers to increase the threshold for unwanted behavior and decrease it for good behavior

- to help the volunteer identify and revert behavioral chains in his/her own life

- to provide specific and individualized nutritional and exercise programs and support.

Volunteers were given the 'LEARN Program for Weight Management 2000' (230) for written reference as this was consistent with our approach. 


\section{Acknowledgements / Dankwoord}

These studies were conducted from August 2000- July 2002 in the Endocrine Research Unit, Mayo Clinic, Rochester, MN, USA, and were supported by grants DK40484, DK45343, DK50456 and R00585 from the U.S. Public Health Service and by the Mayo Foundation. Further personal financial support was kindly given by NovoNordisk Pharma. Eli Lilly Pharma kindly supported the printing of this thesis.

The data processing was done from August 2002- December 2006 in Oegstgeest, Amsterdam (VU Medical Center), Venlo (VieCuri Medical Center), Rochester, MN, USA (Endocrine Research Unit, Mayo Clinic). NMR spectroscopy was done at LipoScience, Inc., Raleigh, NC, USA.

Many people have been helpful in bringing this thesis to a good end with their hard work and/or support and I wish that I could thank them all personally. I will, however, have to restrict myself to giving special thanks to people who were vital in bringing this about.

Firstly and mostly, my mentor, Dr Michael Jensen. Dear Mike, thank you for giving me the opportunity to work at Mayo, and for showing enormous confidence in me from day one. Your coaching during my time in Mayo extended years after my leaving and I have bothered you weekly during our traditional conference calls.

Your personal kindness seemed to be an essential part of your mentorship; this has grown into a dear friendship with you and your wife Teresa. It was an absolute privilege to work with you and I am proud to know you.

Professor Stehouwer: Beste Coen, hartelijk dank voor je hulp en adviezen omtrent het proefschrift en het promoveren. Je supersnelle turn-over van ingediende manuscripten ondanks je drukke tijdschema is fantastisch en een voorbeeld voor alle promotores.

I would like to thank the Members of the Committee, Prof dr. ir. A .M.W.J. Schols (chairman), KN Frayn, PhD MRCPath, Prof.dr.ir WHM Saris, Prof. dr. N C Schaper and Prof. dr. ir. J.C. Seidell, for their willingness to invest their time and expertise in the review of my thesis.

Dr Frayn: dear Keith, I took my first steps in the field of clinical physiology under your supervision. You have shown me that great science, respect and down-to-earthness can and do co-exist naturally. I feel honoured that you took the time and trouble to join this Committee.

The staff of the Mayo General Clinical Research Center: dieticians, lab and nurses, thank you for your assistance in the studies and for making them run as smoothly as they did. Thanks also to Dr Jensen's lab, especially Darlene Lucas en Deb Harteneck for their hard work, particularly at the end of my stay in Rochester. I owe thanks to Monica Davis and Sue Leachman for secretarial support, and, of course, to the volunteers of our studies for participating. 
Most of all however, Jean Feehan and Barb Norby: I could never have done this without you! Your help and friendship during my 2 years at Mayo were vital in bringing these studies to a good end.

I would like to thank Dr Christina Koutsari for her tireless work on the TLCs and the Am J Phys paper, and Dr Scott Crow, psychiatrist, for creating and helping me with our Behavior Modification program.

My collegues from the VU Medical Center: beste collegae, bedankt voor het daadwerkelijk beschermen van de tijd die ik wekelijks mocht inruimen voor het uitwerken van mijn data.

My collegues and partners from the VieCuri Medical Center: beste collegae, bedankt voor de collegialiteit en de steun van de afgelopen 2 jaar.

My friends in Holland, the USA, the UK and all over the world. It is a rare privilege to have so many special people like you in my life. I wish I could thank you all personally for that! Special thanks in the context of this thesis, however, goes out to my dear friends Karen Modesto and Håkan Karlsson who were always there for support, cheering up and great laughs despite the geographical distance between us. Cynthia Tie, 'my big sister in the USA', still going strong after all those years: it was great to have you there for moral support and help in the USA, even though we never saw each other during my 2-year stay in Rochester!

Thea Janssen, mijn Heldense vriendin: dankzij jou en de andere leden van ToBeContinued heb ik relatief snel kunnen inburgeren in Limburg ('limburgeren'). Bedankt voor je warme vriendschap en je inzet als 'hulpnymf'.

Mijn paranymfen Judella Daal en Sylvie vd Haar: jullie vriendschap op afstand lijkt ondanks de lage contactfrequentie alleen maar te groeien in de afgelopen 12 respectievelijk 18 jaar! Bedankt dat jullie er voor me zijn in deze bijzondere rol.

Geen bloedverwanten, wel familie: velen van jullie hebben bijgedragen aan wie ik ben en hebben mij het zelfvertrouwen te geven om de wereld in mijn eentje te attakkeren. In het bijzonder wil ik mijn ooms Abdu en Yusuf en mijn tante Gerda noemen: jullie trouwe liefde en steun is onvermoeibaar en van onschatbare waarde.

Majidah en Tariq, mijn zielsverwanten en overige twee musketiers. Waar zou ik zijn zonder jullie? Wij begrijpen elkaar zonder woorden- die heb ik nu dan ook niet nodig!

Ziyad, Faisal, Iman, Maysoon en Hisham: jullie zijn de versieringen van mijn leven. 5 kleine lichtpuntjes die bij mij altijd een glimlach tevoorschijn weten te toveren.

Papa en mama: jullie steun en liefde zijn de ruggengraat van mijn carrière. Papa, dit boek is voor een groot deel voor jou; mama, dit boek is voor een groot deel van jou.

And last, but certainly not least: Rolf, my sweet love, thank you for making my life complete. 


\section{Curriculum Vitae}

The author of this thesis was born in Leiden, The Netherlands, on January $14^{\text {th }}, 1970$. She finished high school at the Gymnasium in Leiden in June 1988. She then studied medicine at Leiden University, where she graduated for both theoretical exams (Propaedeuse and Doctoraal (Masters)) cum laude. Subsequently, she enrolled at the Subfaculty of Biochemistry of Oxford University, United Kingdom, where she started a research project on adipose tissue metabolism in vivo. There, the basis was set for her love of research under influence of her supervisor, Dr Keith N. Frayn.

Nevertheless she returned to Leiden to finish her registration and licensing as a physician (1994). When her plans to perform a doctorate thesis at Oxford did not obtain the necessary funding, she decided to specialize in internal medicine at Groningen University, The Netherlands, but was drawn back to research in August 2000, when she moved to Rochester, Minnesota, USA to start a 2-year research fellowship at the Mayo Clinic, Endocrine Research Unit on the effect of diet and exercise versus pioglitazone on intramuscular triglyceride metabolism under the mentorship of Dr. Michael D.Jensen.

Meanwhile she graduated for the USMLE (United States Medical Licensing Exam) in November 2000 and registered as a consultant in internal medicine in April 2001.

In the year 2002 she returned to The Netherlands, where she started work as a Consultant in Internal Medicine at the VU Medical Center in Amsterdam, until taking up her present position at the VieCuri medical center in March 2005.

\section{Publications (other than presented in this thesis)}

\section{Bibliography}

S Shadid and MD Jensen 'Nutrient Partitioning'; Book chapter for:'Handbook of Obesity: Etiology and Pathophysiology', Second Edition', edited by George A Bray \& Claude Bouchard. Informa Healthcare; December 9, 2003, ISBN-10: 0824709691; ISBN-13: 9780824709693

\section{Original papers}

$S$ Shadid and MD Jensen 'Angioneurotic edema as a side effect of pioglitazone' Diabetes Care 2002.Feb. 25(2): 405

S Shadid and JC ter Maaten 'Sarcoidosis—a great mimicker' J Int Med 2002; Feb; 251(2): 174-6 
KN Frayn, S Shadid, R Hamlani, SM Humphreys, ML Clark, O Boland and SW Coppack; 'Regulation of the flow of fatty acids in human adipose tissue in vivo in the postabsorptive to postprandial transition'. Am J Physiology 266 (Endocrinology and Metabolism 29): E308 E317, 1994

BA Fielding, SM Humphreys, S Shadid and KN Frayn; 'Monoacylglycerol isomerisation is not rate limiting for triacylglycerol hydrolysis by adipose tissue lipoprotein lipase in the postprandial state in vivo'. Endocrinology and Metabolism, 1995, 2, 13-17

BA Fielding, SM Humphreys, S Shadid and KN Frayn; Oxford Lipid Metabolism Group. 'Plasma mono-, di-, and triglycerol measurements in a study of fat uptake by human adipose tissue in vivo'. Biochem. Soc. Transact. 1995,23:487S. 